Florida International University FIU Digital Commons

6-20-2006

\title{
Subdural electroencephalogram analysis for extracting discriminating measures in epileptogenic data
}

Mercedes Cabrerizo

Florida International University

DOI: $10.25148 /$ etd.FI14052524

Follow this and additional works at: https://digitalcommons.fiu.edu/etd

Part of the Electrical and Computer Engineering Commons

\section{Recommended Citation}

Cabrerizo, Mercedes, "Subdural electroencephalogram analysis for extracting discriminating measures in epileptogenic data" (2006). FIU Electronic Theses and Dissertations. 1960.

https://digitalcommons.fiu.edu/etd/1960 
FLORIDA INTERNATIONAL UNIVERSITY

Miami, Florida

\section{SUBDURAL ELECTROENCEPHALOGRAM ANALYSIS FOR EXTRACTING DISCRIMINATING MEASURES IN EPILEPTOGENIC DATA}

A dissertation submitted in partial fulfillment of the

requirements for the degree of

DOCTOR OF PHILOSOPHY

in

ELECTRICAL ENGINEERING

by

Mercedes Cabrerizo 
To: Dean Vish Prasad

College of Engineering and Computing

This dissertation, written by Mercedes Cabrerizo, and entitled Subdural Electroencephalogram Analysis for Extracting Discriminating Measures in Epileptogenic Data, having been approved in respect to style and intellectual content, is referred to you for judgment.

We have read this dissertation and recommend that it be approved.

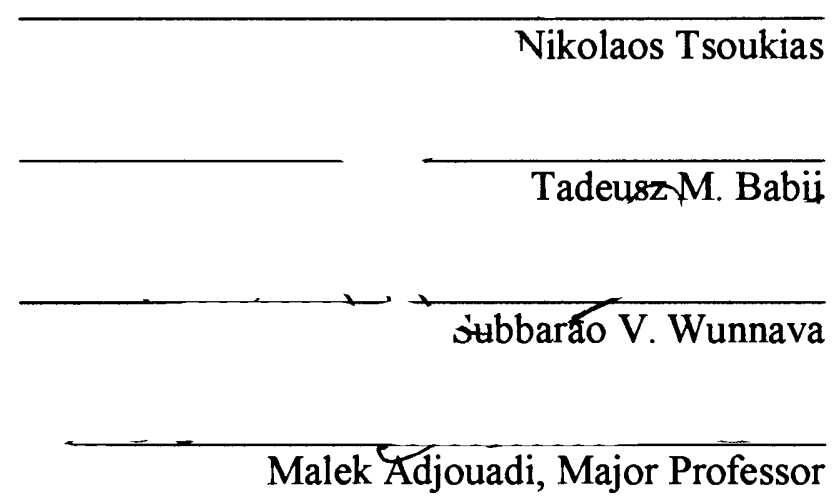

Date of Defense: June 20, 2006

The dissertation of Mercedes Cabrerizo is approved.

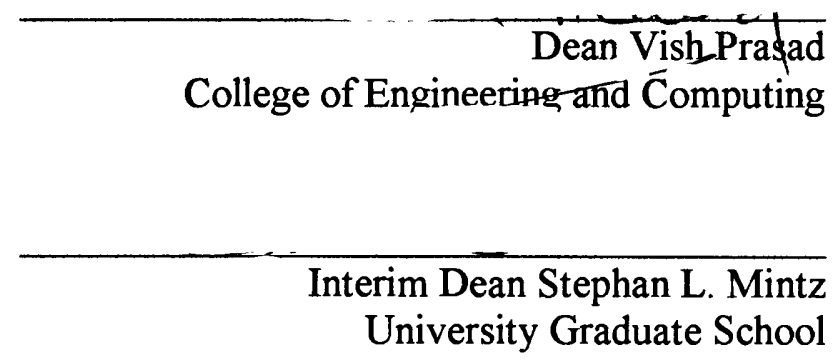

Florida International University, 2006 
C Copyright 2006 by Mercedes Cabrerizo

All rights reserved. 


\section{DEDICATION}

I dedicate this dissertation to my family. Without their patience, understanding, support, and most of all love, the completion of this work would not have been possible. 


\section{ACKNOWLEDGMENTS}

I wish to thank the members of my committee for their support and patience, Dr. Nikolaos Tsoukias, Dr. Tadeusz M. Babij, and Dr. Subbarao V. Wunnava, for their valuable comments for my research. Dr. Prasanna Jayakar for the many discussions we had and his insightful ideas, and comments that made this dissertation more concise. I would like to give special thanks to Dr. Ilker Yaylali, who also provided me with all the needed literature about my research. I would like to express my gratitude to Dr. Melvin Ayala for offering me excellent advice for this research. I am most grateful and forever indebted to my major professor Dr. Malek Adjouadi. He has been extremely helpful in identifying my dissertation topic and publishing the papers. Working with him has been a great pleasure for me. He is the professor who truly made a difference in my life. I owe him my eternal gratitude.

My sincerest appreciation to the National Science Foundation and the Office of Naval Research for their support provided under grants EIA-9906600, N00014-99-10952, HRD-0317692 and CNS 042615. I also thank the National Science Foundation Graduate Fellowship Program for the prestigious fellowship that allowed me to pursue my Ph.D. studies. The support of Miami Children's Hospital has been critical in carrying out the experimental work of this dissertation.

I thank my family for their unconditional support that keeps me persevering in my work. My thanks also go to Daniel for his encouragement and unconditional support. 


\section{ABSTRACT OF THE DISSERTATION}

\section{SUBDURAL ELECTROENCEPHALOGRAM ANALYSIS FOR EXTRACTING}

\section{DISCRIMINATING MEASURES IN EPILEPTOGENIC DATA}

$$
\text { by }
$$

\section{Mercedes Cabrerizo}

Florida International University, 2006

Miami, Florida

Professor Malek Adjouadi, Major Professor

This dissertation introduces an integrated algorithm for a new application dedicated at discriminating between electrodes leading to a seizure onset and those that do not, using interictal subdural EEG data. The significance of this study is in determining among all of these channels, all containing interictal spikes, why some electrodes eventually lead to seizure while others do not. A first finding in the development process of the algorithm is that these interictal spikes had to be asynchronous and should be located in different regions of the brain, before any consequential interpretations of EEG behavioral patterns are possible. A singular merit of the proposed approach is that even when the EEG data is randomly selected (independent of the onset of seizure), we are able to classify those channels that lead to seizure from those that do not. It is also revealed that the region of ictal activity does not necessarily evolve from the tissue located at the channels that present interictal activity, as commonly believed.

The study is also significant in terms of correlating clinical features of EEG with the patient's source of ictal activity, which is coming from a specific subset of channels 
that present interictal activity. The contributions of this dissertation emanate from (a) the choice made on the discriminating parameters used in the implementation, (b) the unique feature space that was used to optimize the delineation process of these two type of electrodes, (c) the development of back-propagation neural network that automated the decision making process, and (d) the establishment of mathematical functions that elicited the reasons for this delineation process. 
I. INTRODUCTION

II. INTERICTAL AND ICTAL EEG SIGNALS AND THEIR CLINICAL

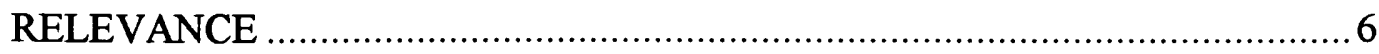

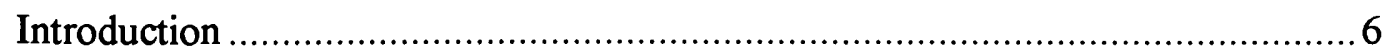

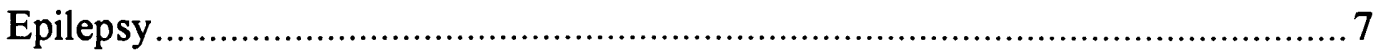

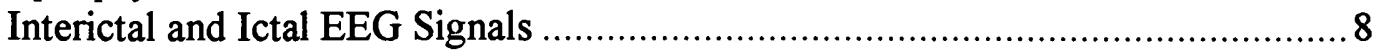



Clinical Criteria Characterizing Interictal Spikes.......................................... 10

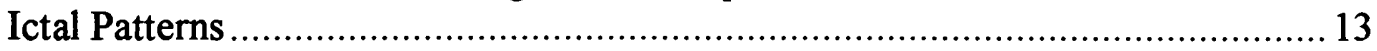

Frequency Contents of the EEG and its Relation to Coherence........................ 14

Advantages of EEG in Comparison to High Technology Brain

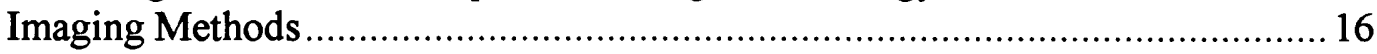

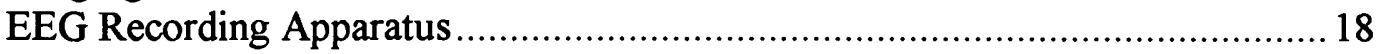

III. INTERICTAL SPIKES DETECTION ALGORITHM THROUGH

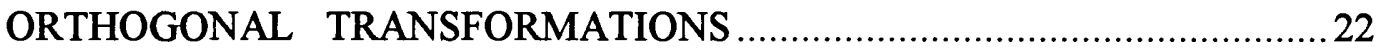

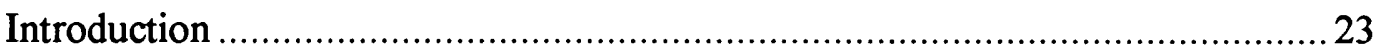

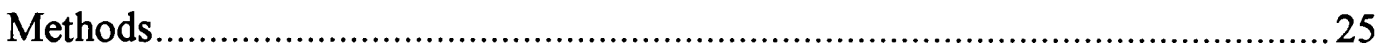

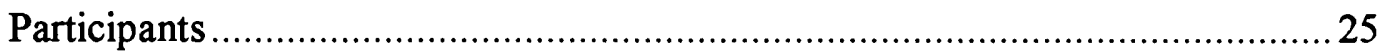

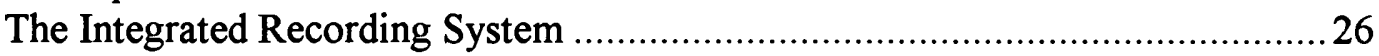

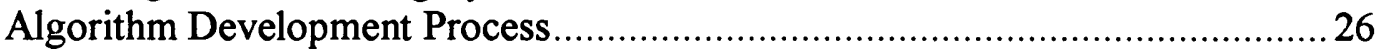

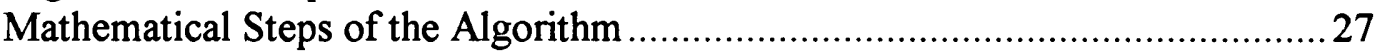

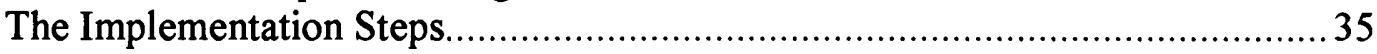

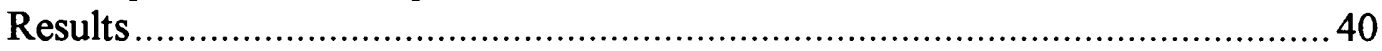

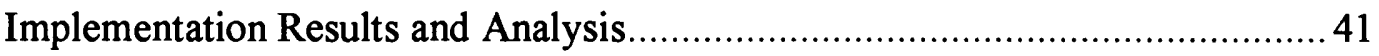

Resolving Contentious Issues of Artifactual Data...........................................47

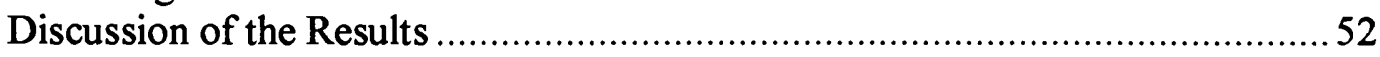

IV. PRELIMINARY EEG DATA MANIPULATION WITH THE PURPOSE OF FINDINGS KEY PARAMETERS TO ANALYZE THE SIGNALS ….............55



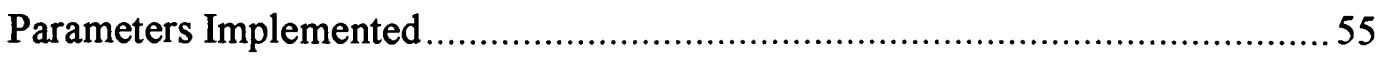

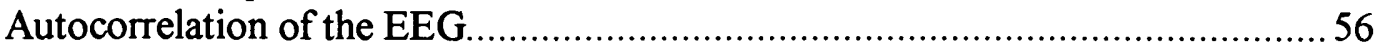



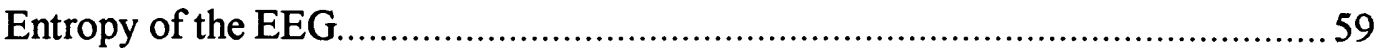

Minimum, Maximum and Average Values of the EEG Signals .......................61

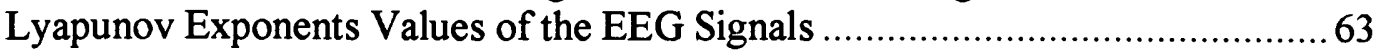

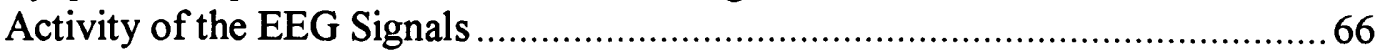

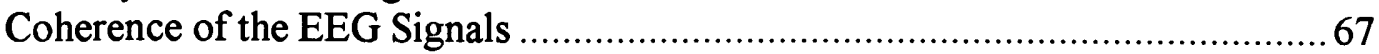

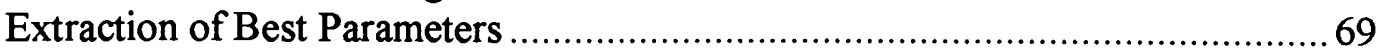




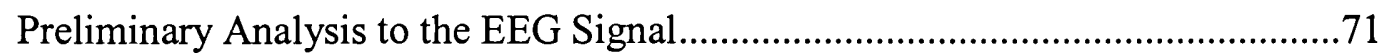

Walsh Transform Applied to the EEG as a Pre-filtering Step ...............................73

Interpretation of the Key Parameters Implemented in this Study...........................75

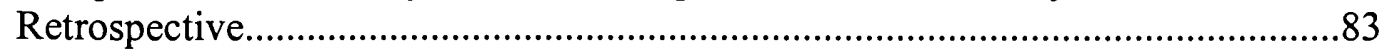

V. INTERICTAL EEG DATA PROCESSING AND RESULTS.............................84

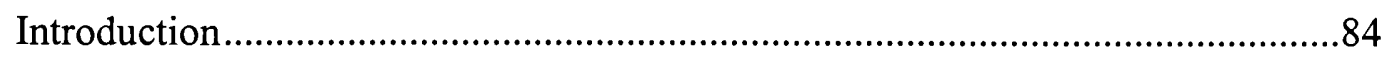

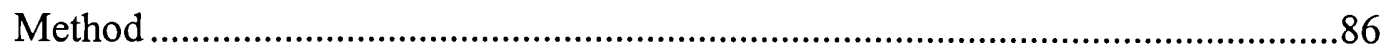

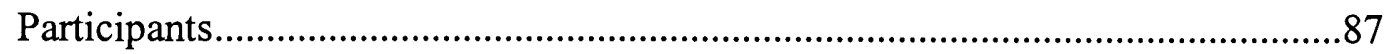

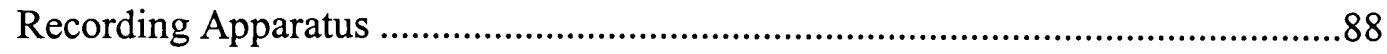

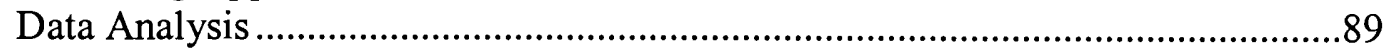

Development Steps of a Novel Classification Algorithm-A Synopsis .................90

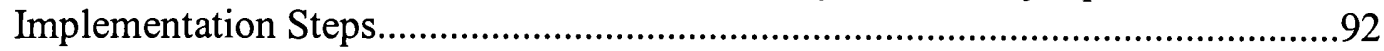

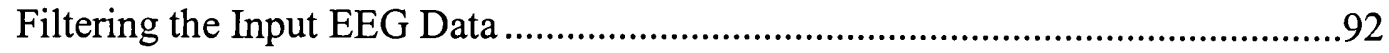

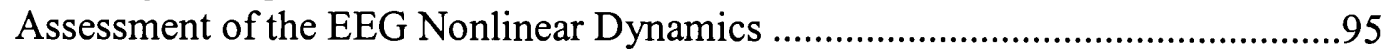

Regression Line Approximations ….................................................................99

Neural Network Structure for Linear Classification ...........................................99

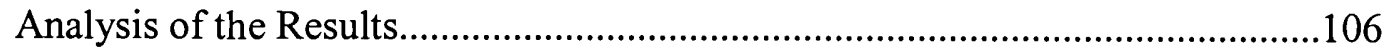

Results of Case with Overlapping (Patient \# 1).................................................116

Dynamics of Correlation Integral, Mobility, and Complexity as

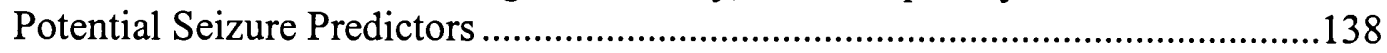

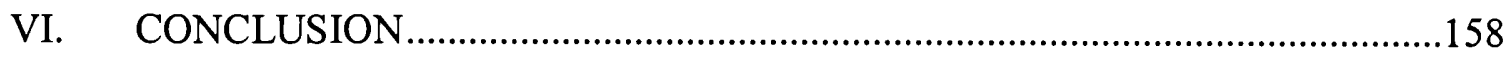

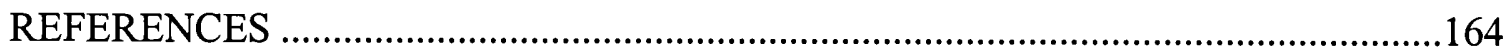

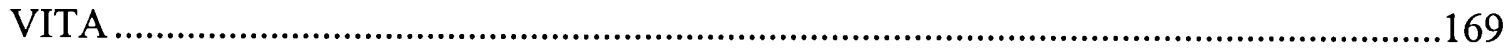




\section{LIST OF TABLES}

TABLE

PAGE

2.1. XLTEK system technical specifications

3.1. Results for both training and testing sets for spike identification

3.2. Results for both training and testing sets for spike identification

3.3. Sensitivity $(S)$ and precision $(P)$ measurements for the phases of training and testing when two experts annotated the spikes.

3.4. Sensitivity $(S)$ and precision $(P)$ measurements for the phases of training and testing when three experts annotated the spikes.

5.1 Patient's clinical information

5.2. Weights of the decision functions for each parameter for patient 1 108

5.3. Percentage of misclassification 109

5.4. Confusion matrix and meaning of its entries 114

5.5. Performance evaluation for classification based on complexity for patient 1 , showing an average misclassification error of $33.33 \%$

5.6. Confusion matrices for all EEG segments of worst case scenario (Patient 1) based on complexity

5.7. Performance evaluation for classification based on correlation for patient 1 , showing an average misclassification error of $25.00 \%$

5.8. Confusion matrices for all EEG segments of worst case scenario (Patient 1) based on correlation

5.9. Performance evaluation for classification based on mobility for worst case scenario (Patient 1), showing an average misclassification error of $23.81 \% \ldots . .118$

5.10. Confusion matrices for all EEG segments of worst case scenario (Patient 1) based on mobility.

5.11. Sensitivity (S) and precision (P) values case with overlapping (patient 1) ....... 120

5.12. Lower $\Downarrow$ or Higher $\Uparrow$ values of the red with respect to the blue channels 


\section{LIST OF FIGURES}

FIGURE

PAGE

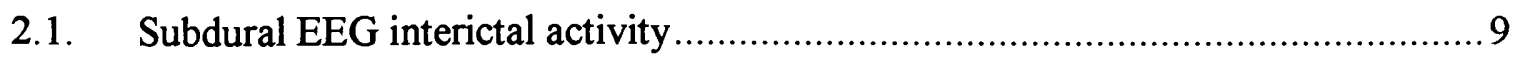

2.2. Simulated spike and it morphological features .............................................. 10

2.3. Interictal subdural EEG data (onset at 240 seconds) ..................................... 13

2.4. Boundaries of Commonly Used Frequency Bands................................... 15

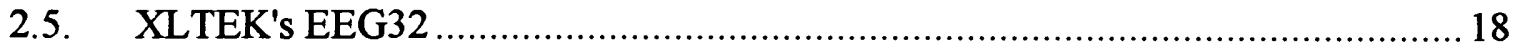

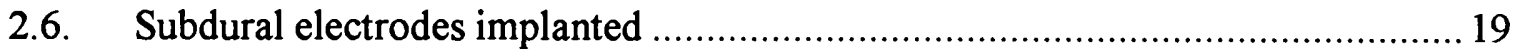

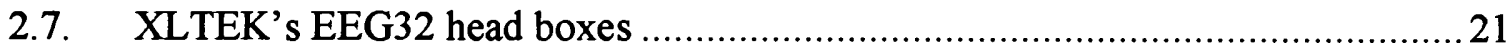

3.1. Contrasting the functionalities of the first-order Walsh operator and the

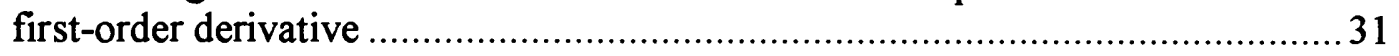

3.2. Contrasting the functionalities of the second-order Walsh operator and the

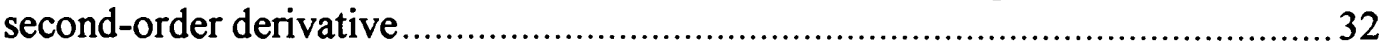

3.3. Comparative results contrasting the errors (by difference) in functionality between the Walsh operators and their analogous mathematical derivatives .......33

3.4. Simulation of interictal spike with anticipated results in $W_{N}^{1}$ and $W_{N}^{2} \ldots \ldots \ldots \ldots . .34$

3.5. Examples of results illustrating the detection of spikes for patient 15 in channels $\mathbf{C} 3$

3.6. Examples of results illustrating the detection of spikes for patient 5 in channels F3

3.7. Examples of results illustrating the detection of spikes for patient 3 in channels FP2

3.8. Examples of results illustrating the detection of spikes for patient 10 in channels FZ

3.9. Results showing multi-channel activity with detected spikes in channels F7, T3, F3 and C3 
3.10. Results obtained when dealing with EKG data - note the periodicity in EKG and how the characteristics sought by the search criteria using the Walsh operators are not met in WT1 and WT2.

3.11. Results obtained when dealing with "Eye Blink" artifact - note how the characteristics sought in WT1 and WT2 are not met, and that WT1 and WT2 have a reversed behavior with respect to a spike (with one peak in W1 instead of 2 and 2 peaks in W2 instead of 1)

3.12. Results obtained when dealing with EMG artifact - note how the characteristics sought by the search criteria using the Walsh operators in WT1 and WT2 are not met



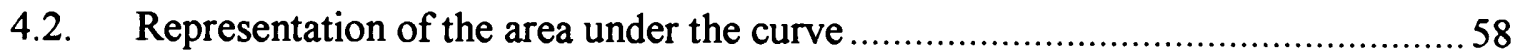

4.3. Representation of the area under the curve over time using equation $4.2 \ldots \ldots \ldots . . .58$

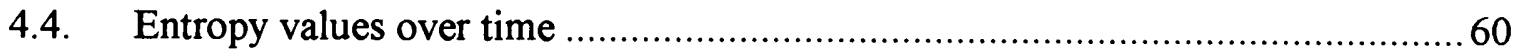

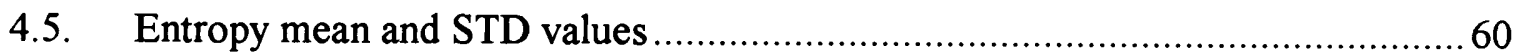

4.6. EEG representation: (a) Minimum (b) Maximum (c) Mean ................................62

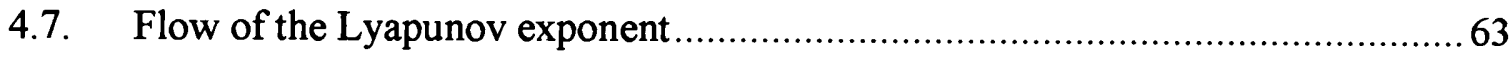

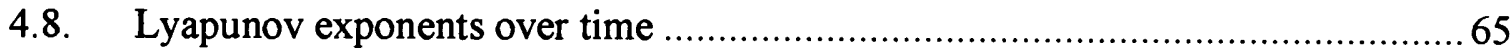

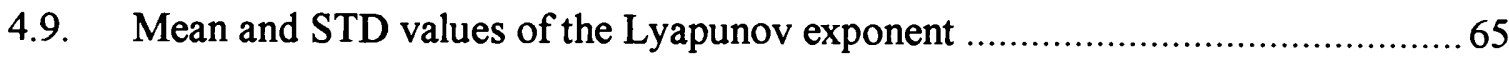

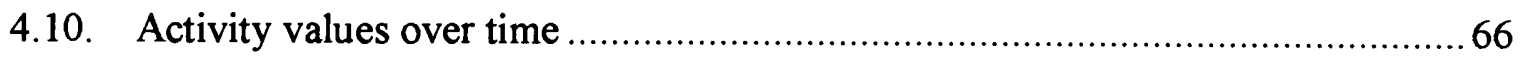

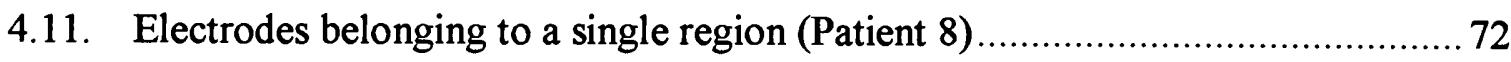

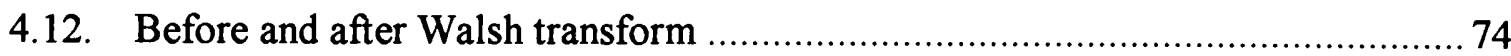

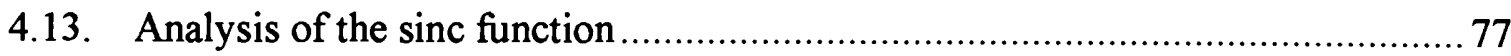

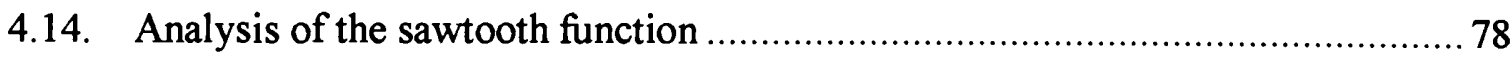

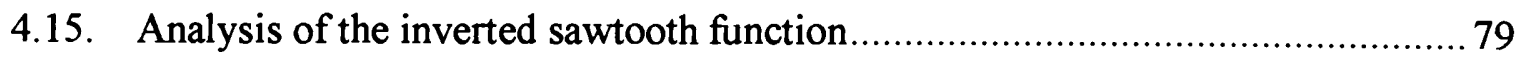

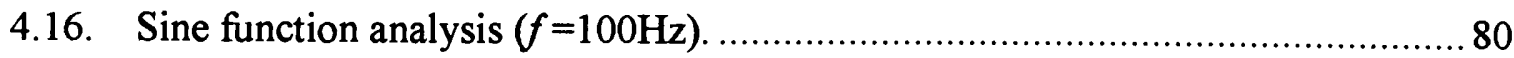


4.17. Sine Function Analysis $(f=200 \mathrm{~Hz})$.

4.18. Two sine functions analyzed

5.1. Interictal EEG data (red electrodes (lead to seizure) \& blue electrodes (do not lead to seizure).

5.2. Intracranial electrodes as placed during surgery

(Courtesy of Miami Children's Hospital) 88

5.3. Flow chart of the different steps in the EEG data analysis process.....................90

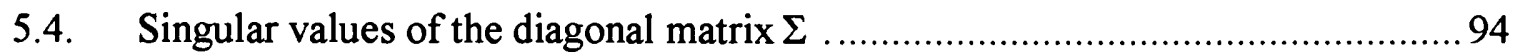

5.5. SVD decomposition applied to the EEG signal (original signal is shown in red and the resulting signal after filtering is shown in blue)

5.6. Clustering of the electrodes based on mean and standard deviation of their correlation values

5.7. Representation of a regression line to represent the trend of the feature 98

5.8. Electrode clusters changing their relative location in the feature vs. time plot

5.9. Network configuration

5.10. Electrode representation in the feature space

5.11. Representation of a weight $w_{j k}$ between two neurons $\mathrm{N}_{\mathrm{j}}$ and $\mathrm{N}_{\mathrm{k}}$, showing neuron outputs, targets and error

5.12. Complexity results for patient 1 (case with overlapping)

5.13. Complexity results for patient 10 (case with no overlapping)

5.14. Misclassification rates for the 3 classifiers of case with overlapping (Patient 1)

5.15. Complexity results for patient 4

5.16. Complexity plots for patient 1 (case with overlapping)

5.17. Complexity results for patient 3 
5.18. Complexity results for patient 2

5.19. Complexity results for patient 5

5.20. Complexity results for patient 6

5.21. Complexity results for patient 7

5.22. Complexity results for patient 8

5.23. Mobility results (1 hour before seizure)

5.24. Complexity results ( 1 hour before seizure).

5.25. Correlation dimension results ( 1 hour before seizure).

5.26. Mobility 2 minutes before seizure (seizure onset at 120 seconds).

5.27. Complexity 2 minutes before seizure (seizure onset at 120 seconds).

5.28. Correlation 2 minutes before seizure (seizure onset at 120 seconds).

5.29. Correlation results 6 minutes after seizure including seizure onset

5.30. Plot of mobility 8 minutes including seizure onset

5.31. Complexity results 6 minutes after seizure including seizure onset.

5.32. Complexity results for all patients 


\section{LIST OF SYMBOLS}

SYMBOL

PAGE

$\Delta_{t} \quad$ Duration of the spike, equation 2.1

$\Delta_{\mathrm{RP}} \quad$ Rising slope of spike, equations $2.1,2.2$

$\Delta_{\mathrm{PF}}$

Falling slope of spike, equations 2.1, 2.2

$\mathrm{P}_{\mathrm{x}}, \mathrm{R}_{\mathrm{x}}, \mathrm{F}_{\mathrm{x}} \quad$ Latencies in the $x$-axis for points $P, R$, and $F$, equation 2.1

$\mathrm{P}_{\mathrm{y}}, \mathrm{R}_{\mathrm{y}}, \mathrm{F}_{\mathrm{y}} \quad$ Latencies in the $\mathrm{y}$-axis for points $P, R$, and $F$, equation 2.1

$\mathrm{W}(\mathrm{x}, \mathrm{u}) \quad$ Walsh transform matrix

Walsh operator

r

Walsh operator's order

$\mathrm{N}$

Number of samples

FN False negative, equation 3.10 
NS Total number of spikes identified

$\mathrm{r}_{\mathrm{k}} \quad$ Autocorrelation, equation 4.1 .56

$\mathrm{k}$

Increment step, equation 4.1 56

Y

Input EEG data, equation 4.1 56

A

Area under the curve, equation 4.2

$\mathrm{X}$

Input vector, equation 4.2

$\mu$

Average of the sample values, equation 4.2

Shannon entropy, equation 4.3

Number of samples minus the window size

Number of partitions, equation 4.3

Probability of the signal, equation 4.3

Phase Input EEG vector, equation 4.8 and 4.9. .64

$\mathrm{A}_{\mathrm{c}}$ Activity, equation 4.10 .66

$\mathrm{f}_{\mathrm{C} 1}, \mathrm{f}_{\mathrm{C} 2} \quad$ Cut off frequencies, equation 4.11 
$X^{\prime}(w) \quad$ Vector with the fourier values, equation 4.11

$\overline{\mathrm{P}_{\mathrm{r}}} \quad$ Relative mean power, equation 4.12

$\mathrm{fb} \quad$ Voltage for one frequency band, equation 4.12

afb Voltage for all frequency bands, equation 4.12

$\mathrm{K}(\mathrm{f}) \quad$ Coherence, equation 4.13

68

$\mathbf{P}_{\mathrm{xy}}$

Cross spectrum, equations 4.13 and 4.14 68

$P_{x x}, P_{y y} \quad$ Power spectrum, equations 4.13 and 4.14 68

$\mathrm{X}_{\mathrm{i}}^{*}(\mathrm{f}), \mathrm{Y}_{\mathrm{i}}^{*}(\mathrm{f})$ Complex conjugate vectors, equation 4.14 68

$X_{i}(f), Y_{i}(f)$ Frequency components of the EEG input, equation 4.14 68



r

Threshold, equation 4.15 70

$\theta(\mathrm{x}) \quad$ Heaviside unit step function, equation 4.15 70

M(y) Mobility, equation 4.16 71

C(y) Complexity, equation 4.17 71

I Input EEG matrix, equation 5.1 93

$\mathrm{U}, \mathrm{V} \quad$ Orthogonal matrices of the input, equation 5.1 93

$\Sigma \quad$ Diagonal matrix with singular values, equation 5.1 93

$\mathrm{x}_{\mathrm{i}}, \mathrm{y}_{\mathrm{i}} \quad$ Points in the regression line, equation 5.3 98

$\mathrm{x}_{\mathrm{i}}, \mathrm{y}_{\mathrm{i}} \quad$ Points in the regression line, equation 5.3 98

$\mathrm{x}_{\mathrm{i}}, \mathrm{y}_{\mathrm{i}} \quad$ Points in the regression line, equation 5.3 .98

$\alpha, \beta \quad$ Intermediate values, equation 5.3 98 
$\mu_{\xi}, \sigma_{\xi}, \Phi_{\xi} \quad$ Mean, standard deviation and frequency power, equation 5.4 101

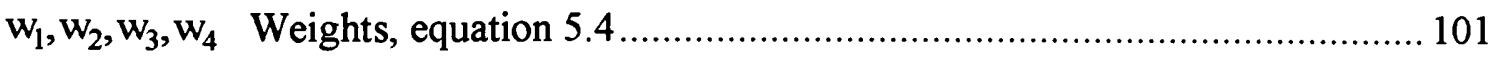

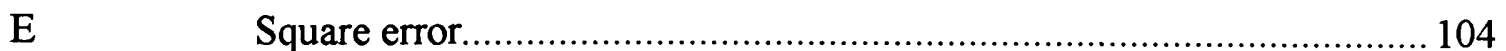

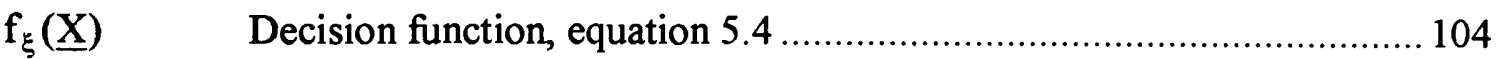

$\Delta \mathrm{w} \quad$ Amount of weight update, equation 5.6 104

$\phi_{\mathrm{k}}{ }^{\prime}\left(\mathrm{x}_{\mathrm{k}}\right) \quad$ First derivative of the transfer function of neuron, equation 5.8 104

$\operatorname{FPr}$

False positive rate. 107

$\mathrm{FNr}$

False negative rate. 108

$\mathcal{E}_{\mathcal{M}} \quad$ Misclassification error, equation 5.15 115 


\section{CHAPTER 1}

\section{INTRODUCTION}

This research work is centered on a study of a human brain malfunction, namely epilepsy. This dissertation introduces a mathematical framework for a new application dedicated at discriminating between electrodes that lead to seizure from those that do not, and yet all the recorded subdural EEG data contain interictal activity, a precursor to seizure. The objective of this study is to resolve this dilemma by: (a) assessing the behavior of all of these channels, which contain interictal spikes that are asynchronous, and (b) determining the key parameters and the related decision functions that permit for such delineation between the two types of electrodes. One nonlinear measure, the correlation integral, and two descriptive measures proposed by Hjorth (mobility and complexity) are used as the key parameters to assess the dynamics of the EEG data prior during and after an epileptic seizure. At the foundation of this mathematical framework is the analysis that is conducted independent of region and time, and where windows of these EEG channels are selected randomly and may or may not contain interictal spikes. An integrated algorithm that exploits these key parameters is developed using regression approximations to view the general EEG behavior, and embedding a novel neural network that yields the decision functions. This algorithm produces accurate results that have been validated by clinical experts at Miami Children's Hospital, and sheds new light on the delineation process between the two types of electrodes. Even the findings related to where the decision functions proved ambiguous or ineffective led to revealing information upon verification in terms 
of the placement of these electrodes and in terms of the type of seizures that a particular subject was experiencing.

Furthermore, the main zones of ictal activity are supposed to evolve from the tissue located at the channels that present interictal activity, but sometimes this was found not to be the case. These findings and resulting observations proved very useful in enhancing our understanding of the dynamics of the human brain by attempting to characterize the common behaviors of interictal EEG channels prior to an ictal activity. These common behaviors as will be demonstrated could potentially lead to the development of new seizure prediction algorithms.

The main objective is to gain a better understanding of the dynamics of the human brain through a study of subdural EEG, with an emphasis on attempting to characterize the common behaviors of interictal EEG channels prior to an ictal activity. The goal of this study is to determine among all of these channels, all containing interictal spikes that are asynchronously, independent of region and time, which are selected randomly (these EEG portions may or may not contain interictal spikes), and yet through the developed algorithm, we are able to classify those channels that lead to seizure and those that did not. A reliable computational tool is implemented in order to detect changes in the EEG channels that suggest the eminence of an epileptic seizure. Here, the investigation is done at the time scale of about 2 to 60 minutes prior or after the seizure onset. This analysis allows thus for the detection of this neurological disorder, and brings out how different patients react prior to a seizure. 
The EEG of epileptic subjects can be divided into two main categories, interictal and ictal. The interictal EEG is the EEG data taken when the patient is not having seizures or in between seizures. Interictal activity is considered to be abnormal if it can occur in a patient with epilepsy in the absence of an actual seizure. The ictal EEG activity on the other hand is when the actual seizure occurs.

The aim is to develop an automated algorithm that determines patterns of dynamic brain behavior during monitoring of epileptic seizures in order to interpret and characterize subdural EEG activities while focusing on trend (like similar behaviors between channels that lead to seizure). The software implemented would be general, both in its implementation structure as well as in characterizing EEG brain behavior, in order to cope with what is expected to be a multitude of elusive brain behavioral characteristics. A main challenge is in the ability to provide meaningful assessments of such subdural EEG behaviors within the context of electrodes that do or do not lead to a seizure.

This research problem is divided in 2 important parts. The first part will describe the behavior of EEG signals during an interictal state. An interictal state is related to activity patterns that refer to spikes or sharp waves notable from normal EEG background activity, which is notorious in human subjects with epilepsy. The second part involves the implementation and processing of the different parameters in order to assess relevant brain changes in the subjects during, at the onset, and after an epileptic seizure. A statistical analysis is conducted and comparative assessments are performed. 
Furthermore, this research is able to determine whether the patient has a consistent source of ictal activity with the most potential to lead to an epileptic seizure, allowing for the epilepsy focus to be located with a higher degree of accuracy.

Epilepsy surgery is now being increasingly performed in children with medically refractory partial seizures. Seizures often arise from extra-temporal sites and are related to a malformed pathologic substrate that is more extensive than the lesion evident on MRI scans. Pre-surgical evaluation is therefore more difficult than in adults and relies heavily on EEG data including interictal spike discharges. The EEG interictal data recorded inside the brain can be processed to define similar patterns evident in those electrodes that lead to a given seizure to further facilitate surgical planning.

The material presented in this dissertation is provided following a chronology that guided the development process of this research work.

Chapter 2 discusses the EEG data content in direct relation to interictal activity information. It provides the details of such a state and the clinical relevance of the epileptic seizure. It also provides the EEG collection process as well as the description of the experimental system used in this research.

Chapter 3 provides a description of the algorithm used in order to detect the interictal spikes. This algorithm formulates characteristics drawn from experimental observation, and develops a new process for exploiting of such characteristics towards accurate identification and localization of the spikes. 
Chapter 4 describes several measurements applied to the EEG data. The EEG data that we used for the development and training of the main classification algorithm presented in chapter 5 was collected from epileptic patients at Miami Children's Hospital. The initial data manipulation performed is explained in details in this chapter.

Chapter 5 provides a comparative analysis between seizure inducing and nonseizure inducing channels applying 3 different key parameters (correlation integral, mobility, and complexity). It also describes the processing steps that were undertaken and the results that were obtained. A detailed flow diagram is presented to show the interrelationship between the different processing methods that were implemented in this dissertation.

Finally, chapter 6 provides concluding remarks on the results obtained and on the procedure that yielded such results. A retrospective of the results obtained is given providing some thoughts on the dilemma that exists between the subjective extractions of similar features based on experience acquired and the attempt of automating such extraction based on mathematical modeling of the interictal EEG data. 


\section{CHAPTER 2}

\section{INTERICTAL AND ICTAL EEG SIGNALS AND THEIR CLINICAL RELEVANCE}

\subsection{INTRODUCTION}

Since the research work of this dissertation involves recording and processing of the Electroencephalogram (EEG), a background on the nature of this signal is necessary. The EEG is generated by changes in the electrical charge of the membrane of cortical nerve cells. These changes fluctuate as a result of impulses arriving from other neurons at contact points, or synapses. This electrical activity creates electrical dipoles, which result from the depolarization and hyperpolarizations of the postsynaptic membrane of the cortical pyramidal neurons. The voltage created by the electrical dipoles, measured on the brain tissue, is the Electrocorticogram (ECoG), with amplitude in the range of millivolts (mv). However, the EEG measured on the scalp is in the microvolts range (generally lies between 10 and 100 micro-volts), since the amplitude of the cortical signal is reduced by the resistance imposed on it by the leptomeninges, cerebrospinal fluid, dura mater, bone, galea, and the scalp (Martini, 2001; Gevins and Remonds, 2000).

EEG has temporal resolution as high as the sampling rate used to record it, which is typically in the range of 1 to $10 \mathrm{msec}$. This is a very high temporal resolution and it allows tracking of the abrupt changes in the signal which are often indicators of EEG abnormalities. However, the major drawback of EEG is its low spatial resolution since EEG signals are recorded only from the predefined locations, where the electrodes are 
placed. Also, EEG lacks spatial specificity, since the electric potential changes are often results of the summation of different remote effects, (Iasemidis, et. al., 1994). This fact plays the crucial role in the source localization procedure because it does not allow simplified assumptions such as the belief that the source of some event is just beneath the electrode from which the particular event was recorded.

The major advantage of EEG remains in its relatively simple technology, and high temporal resolution. This makes EEG a major clinical and research tool for the study of brain functions.

\subsection{EPILEPSY}

Epilepsy is a neurological disorder that causes sudden electrical discharges in the brain referred to as seizures. A seizure represents a sudden, time-limited alteration in function secondary to an abnormal discharge in the central nervous system, (Le Van, et. al., 2001). Epilepsy is not a single disease but rather a sign of underlying brain dysfunctions. It can be defined as a chronic condition during which a patient experiences recurrent seizures. It is very important to distinguish a single seizure, which can be caused by high fever, severe head injury, and lack of oxygen or by number of other factors that can affect the brain enough to cause a seizure, from the epilepsy seizures which are of a recurring nature (Ochi, et. al., 2001; Ebersole, 1997).

Different kinds of seizures determine different types of epilepsy. If the electrical discharges occur in multiple brain areas, they cause generalized seizures. A generalized seizure affects the whole body. In this kind of seizure a person becomes unconscious, 
falls down, and has convulsions. If the electrical discharges occur in just one area of the brain, they cause partial seizures. If a person has a partial seizure, only part of the body is affected. This can be a change in the sense of smell, or the person may feel dizzy. The smallest seizure is called an absence seizure. A person may just seem "out of it" for a moment (Gevins, et. al., 1987).

In most cases the cause of epilepsy cannot be identified. However, where causes can be identified, they may be varied in nature. They include birth injury or congenital malformations due to brain damage, infections, cerebrovascular disease, tumors, neurodegenerative disorders, drugs or toxins, or metabolic disorders.

\subsection{INTERICTAL AND ICTAL EEG SIGNALS}

\subsubsection{INTERICTAL PATTERNS}

EEG signals are the result of the complex neuronal interaction. As illustrated in Figure 2.1, interictal epileptiform activity pattern refers to idiosyncratic waves (spikes or sharp waves) notable from background activity, which is notorious in human subjects suffering from epilepsy (Greenstein, et. al., 2000).

They constitute an abrupt change in voltage, which quickly reverses itself. Spikes may or may not occur repetitively. The shape of the spikes and sharp waves differ significantly within and between patients. The distinguishing pattern between them corresponds to the duration of spikes that is between $20 \mathrm{msec}$ and $70 \mathrm{msec}$, during which 
the sharp peak occurs, and sometimes they are followed by a slow wave (Adjouadi, et. al., 2004; Adjouadi, et. al., 2005 ).

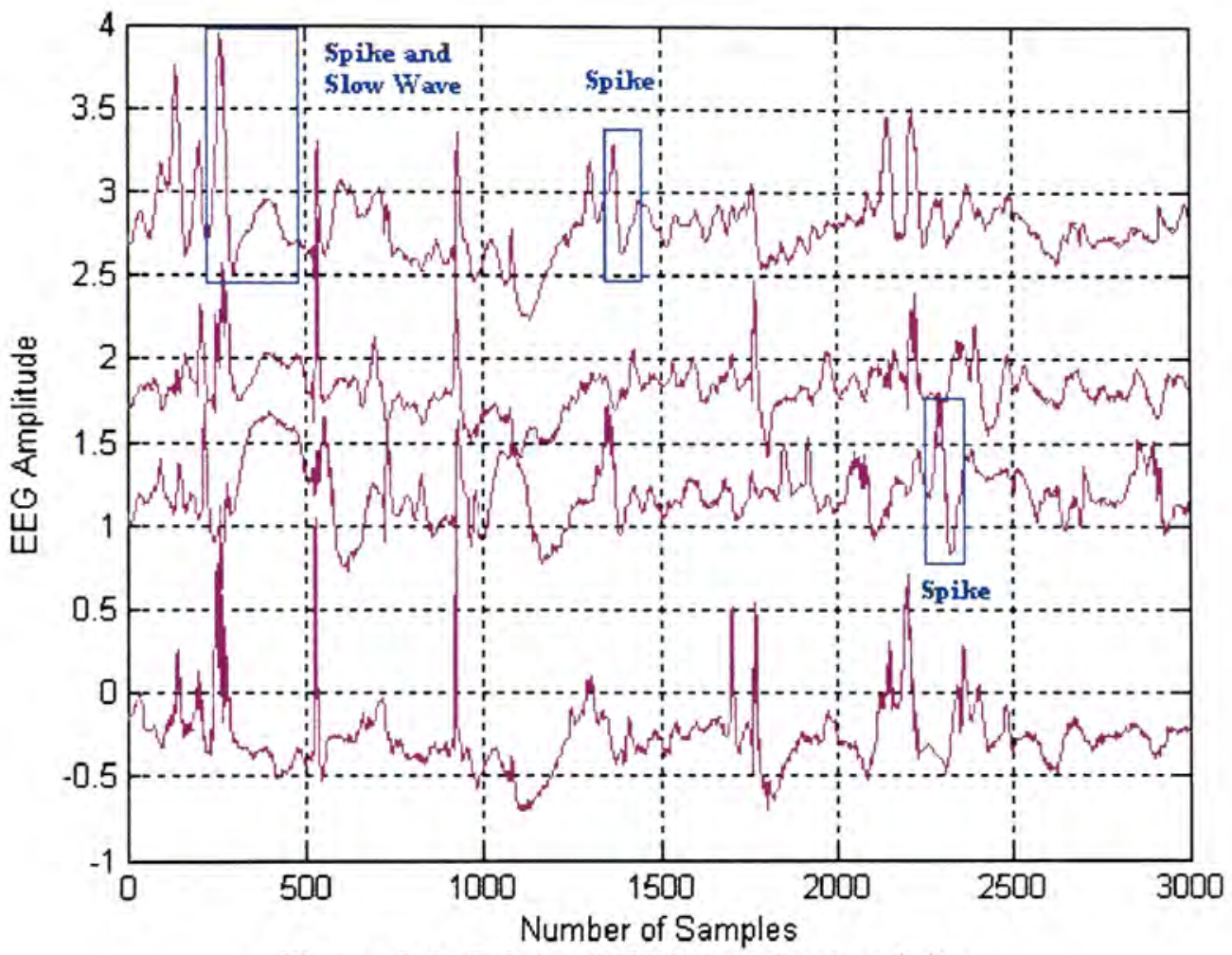

Figure 2.1. Subdural EEG interictal activity.

Note: The 3 events in the squares correspond to spikes and spike and slow wave.

These sharp waves are known to be the indicators of the disease and are present in the EEG recordings of epileptic patients when they do not experience seizures. They originate from the brain areas responsible for generating epileptic activity and are therefore used for source localization of epileptic foci. The pattern of interictal abnormalities can determine if the person has focal or generalized epilepsy. These types of epilepsy have different causes, and may respond to different drugs. 
It is very rare that healthy persons will have epileptiform discharges in the EEG. That is why there is a strong relationship between epileptiform discharges and epileptic seizures. Seizures are characterized by highly abnormal electrical behavior in neurons within epileptogenic regions. There is still no cure for epilepsy. Epilepsy may be treated with drugs, a special diet or surgery. There are many different drugs that can help control epileptic seizures such as Chlorpromazine, Fluphenazine, Thioridazine, Haloperidol, among others (Daly, D.D. and Pedley, A., 1997).

\subsubsection{CLINICAL CRITERIA CHARACTERIZING INTERICTAL SPIKES}

A simulated spike waveform is shown in Figure 2.2 in order to provide key assessments of its characterizing features, with an excellent overview provided in (Gevins and Remond, 1987).

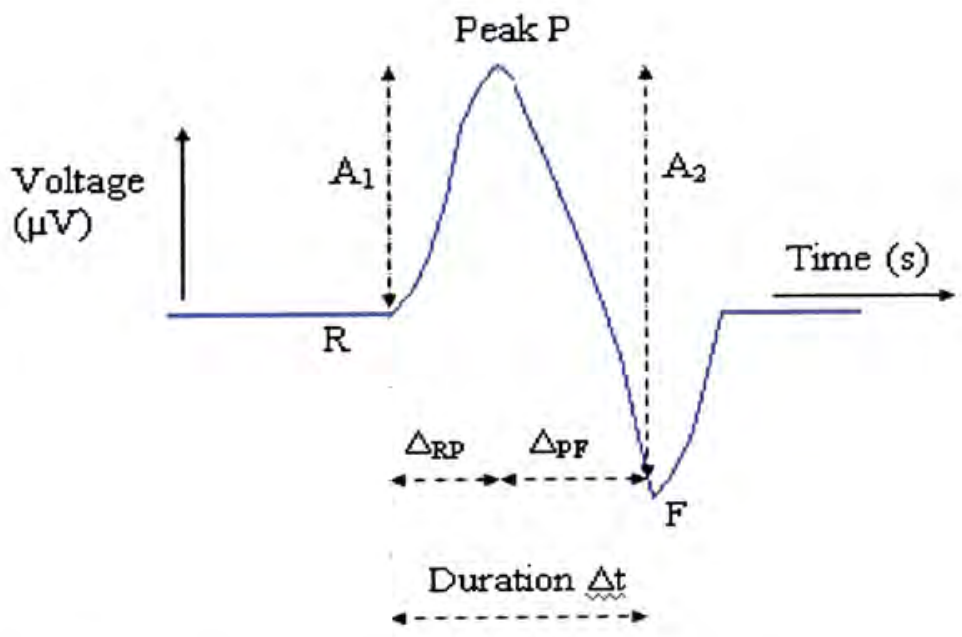

Figure 2.2. Simulated spike and it morphological features. 
With the help of medical experts, the following list of criteria was established as necessary to declare the existence of an interictal spike, defined as a waveform $R P F$, with two half waves $R P$ and $P F$ :

1. Sharpness of a spike is continuous in both narrow and wide intervals of observation.

2. The rising and falling slopes of the spike are both steep. As illustrated in Figure 1, the rising slope $m_{R P}$ is measured from the first trough $R$ to the peak of the spike, and the falling slope $m_{P F}$ is measured from the peak to the second trough $F$.

3. A sharp peak $P$ characterizes the spike, which is due to a sudden change in polarity of the voltage signal recorded. This sharpness may occur in both the time and spatial domains.

4. A spike is estimated to have a total duration of 20 to 70 milliseconds (ms). The total duration of the spike, $\Delta_{t}$, is measured from $R$ to $F$, as the sum duration of the two half waves. $P_{x}, R_{x}$, and $F_{x}$ denote the respective latencies in the $x$-axis for points $P, R$, and $F$.

$$
\Delta_{t}=\Delta_{R P}+\Delta_{P F}, \text { Where } \Delta_{R P}=P_{x}-R_{x} \text { and } \Delta_{P F}=F_{x}-P_{x}
$$

5. The two half waves are observed to satisfy the condition that their absolute difference is less than or equal to their calculated average (Jayakar, et. al., 1989). This implies that the duration of the shorter half wave must be at least one third of that of the longer half wave.

$$
\left|\Delta_{R P}-\Delta_{P F}\right| \leq \frac{\left(\Delta_{R P}+\Delta_{P F}\right)}{2}
$$


6. The amplitude of a spike is greater than 20 microvolts $(\mu \mathrm{V})$. The amplitude $A_{a}$ is defined as:

$$
A_{a}=\left(A_{1}+A_{2}\right) / 2
$$

with $A_{1}=P_{y}-R_{y}$, and $A_{2}=P_{y}-F_{y}$

And where, $P_{y}, R_{y}$, and $F_{y}$ denote the respective latencies in the $y$-axis for points $P, R$, and $F$. In addition, the downward deflection voltage $A_{2}$ is usually larger than the upward deflection voltage $A_{1}$, and satisfying the condition:

$$
\frac{1}{4} \leq \frac{A_{1}}{A_{2}} \leq 2
$$

7. The maximum amplitude of a spike is at least 1.5 times larger than that of the background signal, where the background signal may be defined as the EEG activity lasting twice the duration of the spike (assumed in this case to be $140 \mathrm{~ms}$ ) at either side of a potential spike.

8. Multi-channel activity may be reported, where one spike observed in a given channel may also be observed in another neighboring channel. In other words, spikes do not occur in isolation. This can be assumed provided that the inter-electrode spacing of the recording system used is small. Neighboring channels are identified as those that are of closest physical proximity, in direct relation to the system's electrodes configuration used (Ochi et al., 2001). 
9. The existence of artifacts (spike-like transients) undermines the identification of spike, and therefore such artifacts must be accounted for automatically by the devised algorithm, in order to minimize the false detection rate.

10. A slow wave may follow a spike. This characteristic, not always present, may be used only to augment the certainty in identifying a spike, but not to undermine it.

\subsubsection{ICTAL PATTERNS}

The ictal EEG abnormalities, as illustrated in Figure 2.3, can show rhythmic activity. Several subdural electrodes leading to seizure are displayed in different colors so the behavior of them before and during the ictal state could be visually appreciated.

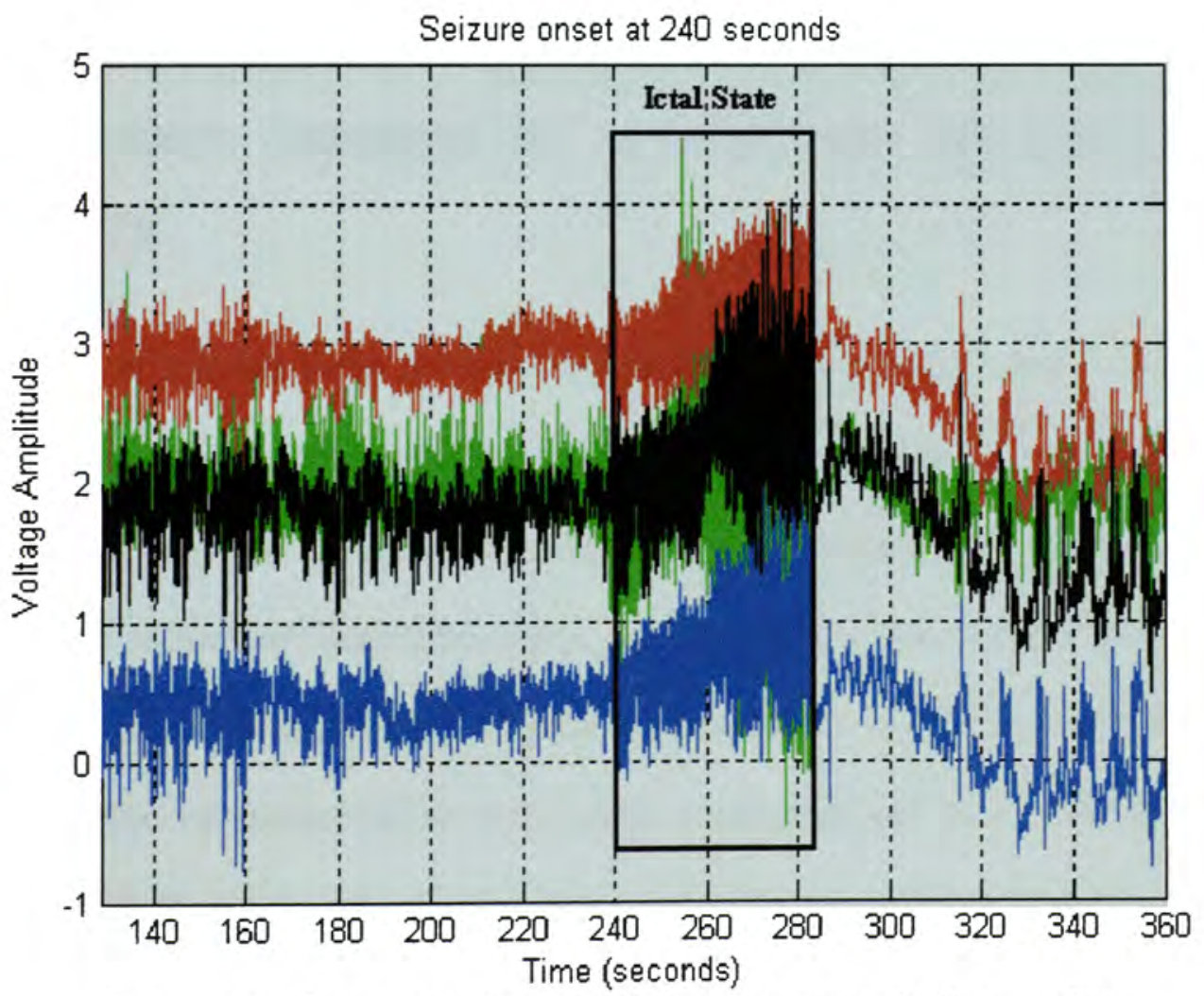

Figure 2.3. Interictal subdural EEG data (onset at 240 seconds). 
The ictal EEG of the person with focal epilepsy will usually show the seizure starting from a specific brain area but it can then spread to involve other areas, or even the whole brain. These patterns can tell the doctor which part of the brain is causing the seizure and how much of the brain becomes involved during it (Frost, 1985).

By looking at the patterns of the EEG in the interictal and ictal stages, the physician can tell if a particular patient has seizures, can determine the type of epilepsy the patient has, and localize the part of the brain that is causing the seizures. This information can help physicians decide which medication is appropriate to treat the seizure. In extreme cases, where no treatment is helpful, and the area that causes the seizures is determined, then the patient may be eligible for neurosurgical treatment (Geiger, L.R. and Harner, R.N. 1978).

\subsection{FREQUENCY CONTENTS OF THE EEG AND ITS RELATION TO} COHERENCE

EEG of humans either at rest or awake commonly contains rhythmical potential changes. These rhythms are called brainwaves and they are measured in frequency (speed of electrical pulses) and in amplitude (intensity of the brainwave). Frequency is measured in cycles per second, or Hertz $(\mathrm{Hz})$. Thus, one frequency is one cycle, or one pulse per second. The frequencies of the EEG signal range from 0.5 to $100 \mathrm{~Hz}$, depending on the degree and type of activity of the brain, such as alertness and sleep. Electrical activity emanates from the brain in the form of brainwaves. There are four brainwave states that range from the low amplitude/high frequency Beta to the high amplitude/low frequency 
Delta. Research has shown that according to the activity level of an individual, one brainwave state may be dominant at any given time, even though all brainwave states will remain present at minimal levels. The frequency of EEG waves is divided into four groups of frequency bands. These divisions are shown in Figure 2.4.

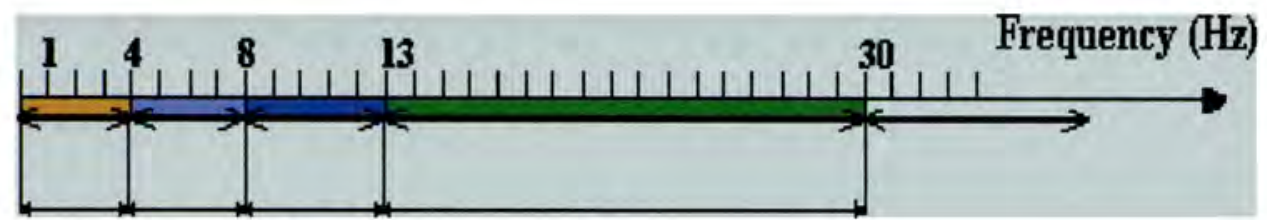

Delta Theta Alpha

$1000 \quad 250 \quad 125$

\section{Beta}

77 ms (Wave Length)

Figure 2.4. Boundaries of commonly used frequency bands.

The four common brain waves are alpha, beta, delta and theta. Alpha waves are those between 8 and $13 \mathrm{~Hz}$. They are the major rhythm seen in normal relaxed adults, and occur whenever the person is alert, but not actively processing information. Beta activity is 'fast' activity. It has a frequency of $13 \mathrm{~Hz}$ and greater. It is generally the dominant rhythm in those who are alert or anxious or who have their eyes opened, and are listening and thinking during analytical problem solving, judgment, decision making and processing information. The lowest frequencies are delta, these are less than $4 \mathrm{~Hz}$, and occurs in deep sleep. It reflects unconscious mind. Theta activity has a frequency of 4 to $8 \mathrm{~Hz}$ and is considered as slow activity. Theta waves are strong during internal focus and meditation (Cabrerizo, et. al., 2005; Popescu, 1998). 
The study of the coherence as a function of frequency is very useful when analyzing disease states such as seizures. Coherence is an amplitude independent measure of phase synchrony between EEG signals. It reflects functional interregional coupling that primarily depends on structural connections. The coherence values are interpreted in terms of differences in connectivity between brain structures. Depending on the state of the EEG, some regions are more synchronized than others depending of the frequency range analyzed.

\subsection{ADVANTAGES OF EEG IN COMPARISON TO HIGH TECHNOLOGY BRAIN IMAGING METHODS}

EEG possesses a high temporal resolution and is simple to use. EEG can reveal one of the main parameters of the neural activity in the brain, which is the rhythmic property that reflects the essence of neural excitation. Therefore while recording electrical (as well as magnetic) field patterns; the physiologist has access to the actual mechanisms of the brain information processing.

Thorough EEG studies can lead to the discovery of the functional representation of human brain processes, revealing not only in what region of the cortex, but how a disease is distributed in the brain by assessing the make up of its pattern activities. Thus, EEG, which previously was treated as a result of simple and sometimes sporadic summation of the activity of enormous masses of neurons, is nowadays an efficient tool in exploring the intimate mechanisms of informational processing in the brain. With the advent of even higher resolution in spatial recordings (up to 256 channels) together with 
the newer technological developments, EEG data analysis will see for the near future far greater enhancements. With the research trends favoring the integration of different modalities such as Magnetic resonance Imaging (MRI) and Diffusion Tensor Imaging (DTI), enhanced EEG analysis will only strengthen the validation process in the diagnosis, prognosis, and perhaps prediction of such key events as epilepsy.

Such advances will be most welcome since EEG provides information about the health and function of the brain. The EEG can help diagnose conditions such as epilepsy, brain tumors, brain injury, stroke, which lead to specific metabolic conditions. EEG can help physicians find the source of other problems such as headaches, weakness, blackouts, dizziness, or auditory/comprehension problems. It can also be used in patients, who are deeply unconscious, to distinguish between brain death and reversible conditions.

Sleep deficiency is another frequent disorder that currently affects millions of people of all walks of life. Electroencephalograms have been used to research bright light therapy and its effectiveness in order to alleviate this common problem. In addition, electroencephalography has become a major tool in aiding physicians to look closer at the origins of alcoholism, along with its often-devastating effects.

The diverse examples mentioned above represent only a small number of applications of electroencephalograph-based technology. Today, EEGs are at the forefront of medical diagnosis and treatments. Continual research on possible applications of the EEG signals will assuredly lead to new ways of fighting common and 
other as yet to be understood diseases such as Alzheimer and Parkinson's disease just to name a few.

\subsection{EEG RECORDING APPARATUS}

The EEG data was recorded using XLTEK Neuroworks Ver.3.0.5 equipment as illustrated in Figure 2.5. It was manufactured by Excel Tech Ltd. Ontario, Canada. Sampling frequencies of $500 \mathrm{~Hz}$ and $200 \mathrm{~Hz}$ were used to obtain the digital EEG recordings.

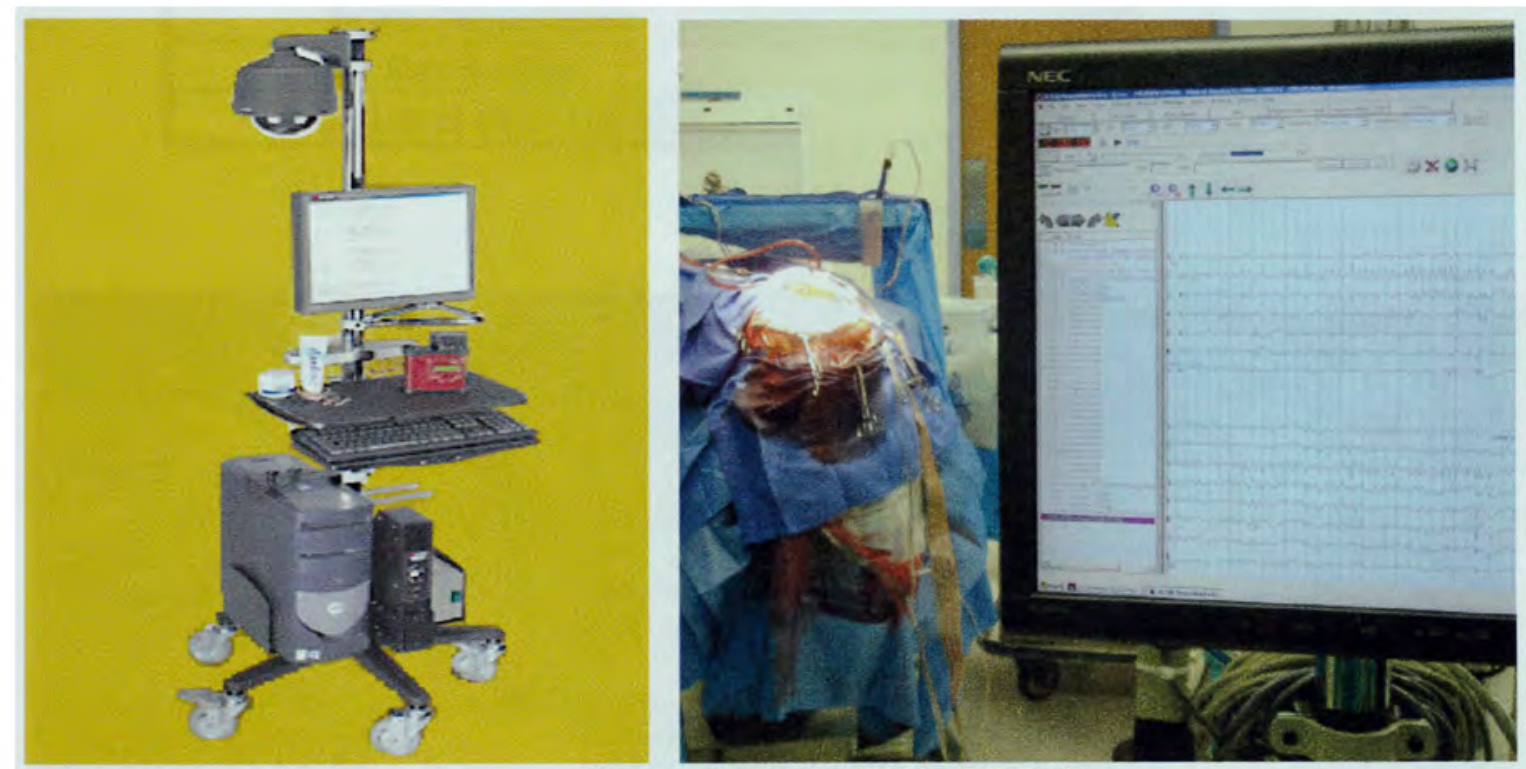

Figure 2.5. XLTEK's EEG32.

Table 2.1 shows the technical specifications. The EEG32 system simultaneously samples and stores data at rates up to $500 \mathrm{~Hz}$ with 22 bit analog conversion.

The EEG is recorded from electrodes placed on key locations inside the brain. Platinum grid electrodes (10-mm inter-contact distance) were surgically implanted as 
shown in Figure 2.6. The maximum number of electrode contacts in each subject was 128. Electrodes consist of a conductor connected by a lead wire and plug to the input of the recording machine. The instrument picks up electrical impulses in the brain and records them.

Table 2.1. XLTEK system technical specifications.

\begin{tabular}{||c|c|}
\hline Sampling Rate & $500 \mathrm{~Hz}$ or $250 \mathrm{~Hz}$ per channel \\
\hline Analog/Digital Converter & 22 bits \\
\hline Voltage Resolution & Accurate to $0.3 \mu \mathrm{V}$ \\
\hline Channels & 32 \\
\hline Maximum Input Range & $\pm 10 \mathrm{mV}$ \\
\hline Input Impedance & $=47 \mathrm{Mohms}$ \\
\hline Bandwidth & 0.1 to $100 \mathrm{~Hz}$ \\
\hline Noise Level (0.1-100 Hz) & $=2 \mu \mathrm{V} \mathrm{p}-\mathrm{p}$ \\
\hline
\end{tabular}
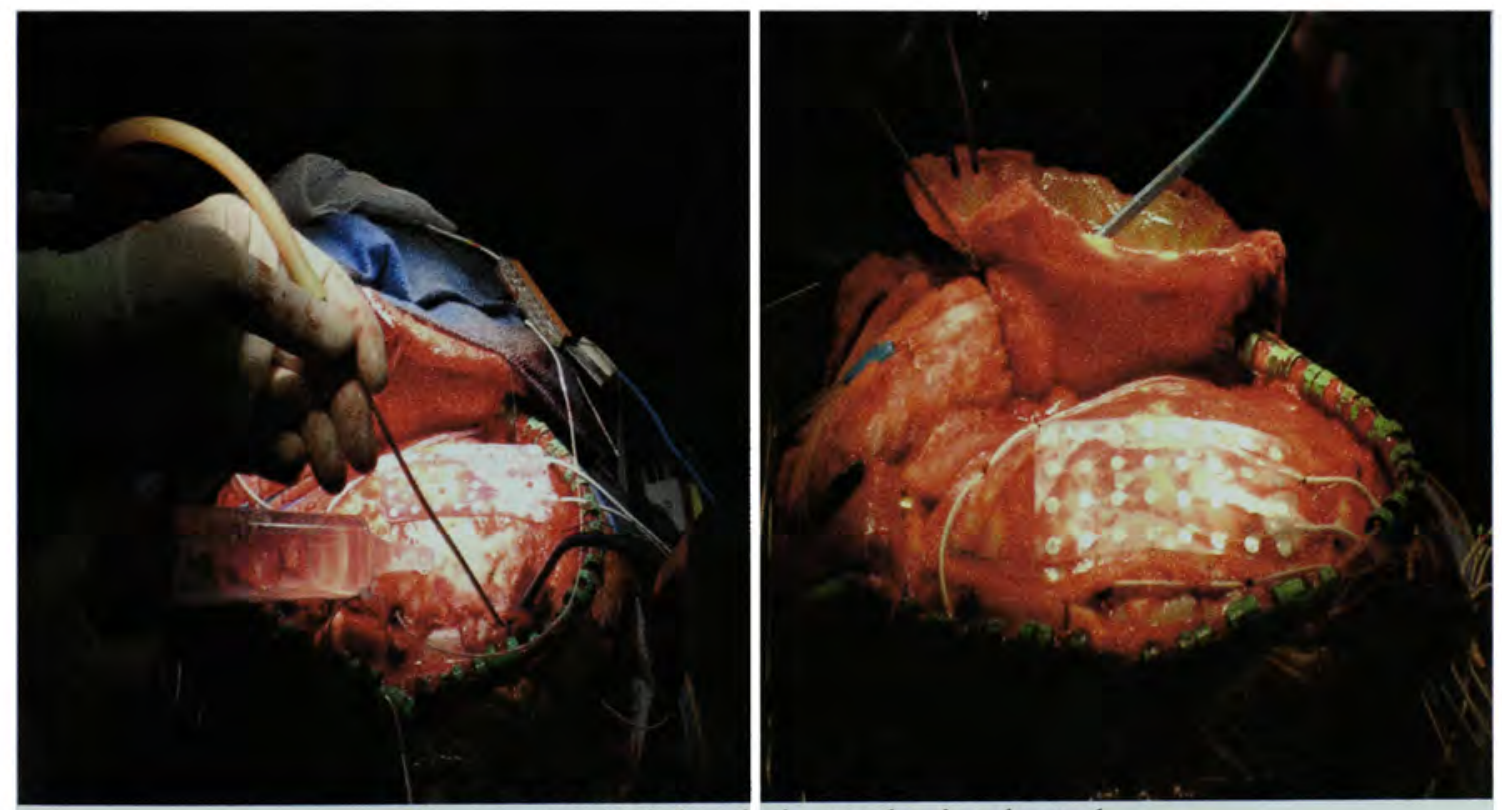

Figure 2.6. Subdural electrodes implanted.

As can be seen from Figure 2.7, the XLTEK hardware setup for the recording of EEG includes two input head boxes connected to the host computer to interface the 
different electrodes to the this computer. The electrodes of the subject are directly connected to head box shown (a). There are different colors in the head box depending on how many electrodes the patient needs. Each color has connections for 32 electrodes. This box from (a) is connected to box (b), and from here to the host computer.

An impedance check can be initiated from the unit itself, and the results are shown with red and green lights directly on the amplifier.

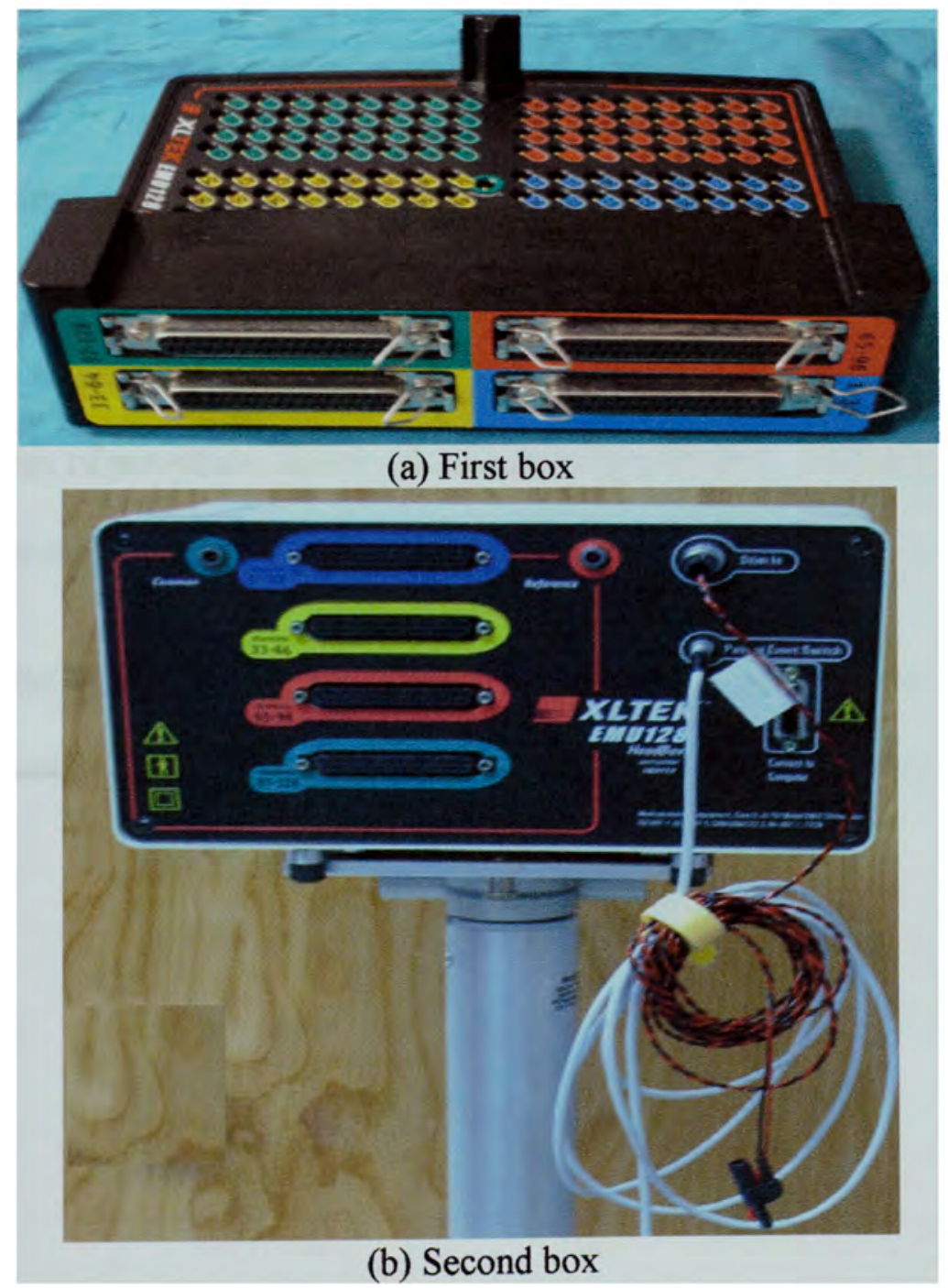

Figure 2.7. XLTEK's head boxes. 
XITEK's amplifier can be used for clinical EEG studies in an outpatient or inpatient setting, long-term EEG monitoring, and ECoG or other operating room procedures.

The electrodes are arranged in general as a particular montage. Montages can be divided into two types: bipolar and referential. Bipolar montages connect pairs of different electrodes to the inputs of the amplifiers. Bipolar montage can localize circumscribed potentials more precisely than with a referential montage; they however, distort the shape and amplitude of potentials.

Referential montage, measures the potentials changes with respect to one common electrode used as a reference. This referential recording can give an undistorted display of the shape of the potential changes. It is useful for recording EEG that has a wide distribution of potentials. However, a disadvantage is that it is usually impossible to find a reference electrode that is completely inactive.

Once the EEG signals are obtained, the next task is to determine which of these EEG signals contain spikes for further analysis. Chapter 3 introduces such work. 


\section{CHAPTER 3}

\section{INTERICTAL SPIKES DETECTION ALGORITHM THROUGH ORTHOGONAL TRANSFORMATIONS}

As a precursor to the classification algorithms that is detailed in Chapters 4 and 5 , which assume all electrodes considered in the analysis have interictal spike activity, this chapter introduces a new method for automatically detecting interictal spikes present in the EEG data.

The first step in this study involves identifying the pertinent electrodes in the overall EEG data that present interictal EEG activity and then to separate the channels in which the seizures occur. A computer program was developed to automatically detect interictal spikes. The results were later validated by the physicians by visual inspection of the analyzed data.

This chapter introduces an integrated algorithm based on the Walsh transform in order to detect interictal spikes and artifactual data in epileptic patients using recorded EEG data. The algorithm proposes a unique mathematical use of Walsh-transformed EEG signals to identify those criteria that best define the morphological characteristics of interictal spikes. EEG recordings were accomplished using the 10-20 system interfaced with the Electrical Source Imaging System with 256 channels (ESI-256) for enhanced preprocessing and on-line monitoring and visualization. The merits of the algorithm are:

(a) its computational simplicity, (b) its integrated design that identifies and localizes 
interictal spikes while automatically removing or discarding the presence of different artifacts such as EMG, EKG, and eye blinks, and (c) its potential implication to other types of EEG analysis, given the mathematical basis of this algorithm which can be patterned or generalized to other brain dysfunctions. The mathematics that were applied here assumed a dual role, that of transforming EEG signals into mutually independent bases and in ascertaining quantitative measures for those morphological characteristics deemed important in the identification process of interictal spikes. Clinical experiments involved 31 patients with focal epilepsy. EEG data collected from 10 of these patients were used initially in a training phase to ascertain the reliability of the observable and formulated features that were used in the spike detection process. Spikes were annotated independently by three EEG experts. On evaluation of the algorithm using the 21 remaining patients in the testing phase revealed a Precision (Positive Predictive Value) of $92 \%$ and a Sensitivity of $82 \%$. Based on the 20 to 30 -minute epochs of continuous EEG recording per subject, the false detection (FD) rate is estimated at 1.8 FD per hour of continuous EEG. These are positive results that support further development of this algorithm for prolonged EEG recordings on ambulatory subjects and to serve as a support mechanism to the decisions made by EEG experts.

\subsection{INTRODUCTION}

The focus of this study is placed on the design of an integrated algorithm for the detection of interictal activity in EEG attributed to focal epilepsy. The detection process is designed such as to allow physicians to make evaluative assessments of epileptic seizures, which in turn will enable targeted treatment. The use of the Walsh transform in 
analyzing epileptogenic data, and the application of its associated mathematical derivations proposed show promise not only for detecting interictal spikes (Adjouadi et al. 2004), but also in characterizing them with quantitative measures. The proposed algorithm is augmented with cautionary measures that identify or filter out artifactual data associated with eye blinks, muscle movement (EMG), and heartbeat (EKG). Processes and methods for the automated detection of interictal activity as presented respond to the diagnostic value of interictal epileptiform activity (Frost, 1985)

Epileptiform activity was assessed in earlier studies (Gotman, 1985; Glover, 1989, Birkemeier, 1978; Jayakar, 1989; and Gevins, 1987) combining an extensive clinical experience. Rule-based detection algorithms have elicited two characteristics that are considered as most reliable in the detection of spikes, and they are the fast rise and decay of the spike, and the sharpness of its peak. The spatio-temporal context is taken into account in several studies using different approaches: context-based detection (Jayakar, 1991; Dingle et al., 1993), state-based detection (Gotman, 1992), neural networks (Hellmann, 1999; Ko and Chung, 2000; Kurth et al., 2000; Tarassenko, 1998, and Ayala et. al, 2004), principles of the wavelet theory (Popescu, 1998; and Calvano et al., 2000, Latka, 2003), and expert systems (Davey et al., 1989; Dingle et al., 1993), to cite a few. An earlier implementation example of the Walsh transform to stereo vision in 2-D images is provided by Adjouadi and Candocia (1994). Also, Barreto et al. (1993) describe a study for the detection of interictal spikes using electrocorticographs and Lagrange derivatives. 
The approach considered in this study is to take EEG data from 10 of the 31 subjects in the study, and use them in a training phase to adjust the mathematical parameters (decision criteria) specifically designed for the task at hand. This phase is used to confirm or slightly redress the criteria established. The testing phase involving EEG data from the other 21 subjects is then performed in order to assess the performance merit of such criteria.

\subsection{METHODS}

\subsubsection{PARTICIPANTS}

Thirty one (31) children with focal epileptic seizures served as subjects for this study. The epileptic patients recorded were either: (a) inpatients at the hospital for longterm EEG monitoring, or (b) outpatients for short-term EEG monitoring sessions. Patients were selected for the study if they were identified by a doctor (MD) as having focal epilepsy. The patients were awake during the recording sessions. However, data in this study were selected randomly, and contained awake, drowsy and also sleep sections. To be able to judge the algorithm's accuracy, the data had body movement, muscle artifacts, eye movement artifacts, and physiologic but non epileptic waveforms such as sleep spindles.

All of the procedures followed strict protocols pursuant to the ethical guidelines and regulatory requirements regarding research involving human subjects. 


\subsubsection{THE INTEGRATED RECORDING SYSTEM}

EEG data was recorded employing the 10-20 electrode placement system interfaced with the Syn-Amps of the Electric Source Imaging system with 256 channels (ESI-256), all under expert clinical supervision at Miami Children's Hospital. The SynAmp amplifiers of the ESI-256 machine were used to allow for optimal online processing of signals for digital filtering, amplification and digitization of the EEG signals, and the transferring of the digitized data to the host computer. The EEG data was collected in continuous mode using a $500 \mathrm{~Hz}$ sampling frequency with $0.1-70 \mathrm{~Hz}$ bandpass filter settings and 22 bits A/D converter. The EEG recording time for each patient varied between 20 to 30 minutes. The EEG sections selected for analysis were those in which artifacts created by the patients physical activity was minimal. Artifacts due to electrical interference by the recording system are not significant with the setup provided by the interface of the ESI-256 machine. Other artifacts, such as eye blinks, EMG, and EKG were recorded. However, by using the ear reference electrodes, recording of EKG artifacts was reduced for most patients.

Based on the morphological aspects of interictal spikes as described in section 2.3.2 of chapter 2 , the development process of the algorithm is described next.

\subsubsection{ALGORITHM DEVELOPMENT PROCESS}

The interesting aspect in this study is that Walsh operators satisfy the important concept of orthogonality and yet an analogy to discrete mathematical derivatives can be 
generalized in terms of their functional behavior. The mathematical steps considered in the development of this algorithm are described in the next section.

\subsubsection{MATHEMATICAL STEPS OF THE ALGORITHM}

The use of the Walsh transform is viewed as an integrated approach to transforming the original EEG signal into orthogonal (mutually independent) Walsh transformed signals under the different sequency orders of the Walsh kernel (Gonzalez and Woods, 1993). An example is given for $8 \times 8$ and $4 \times 4$ Walsh matrices (kernels) where the basis functions satisfy orthogonality in the row and column vectors. The sequency in these ordered forms (ascending sequency) denotes the number of sign changes in each row vector as shown in parenthesis following the last column of each kernel

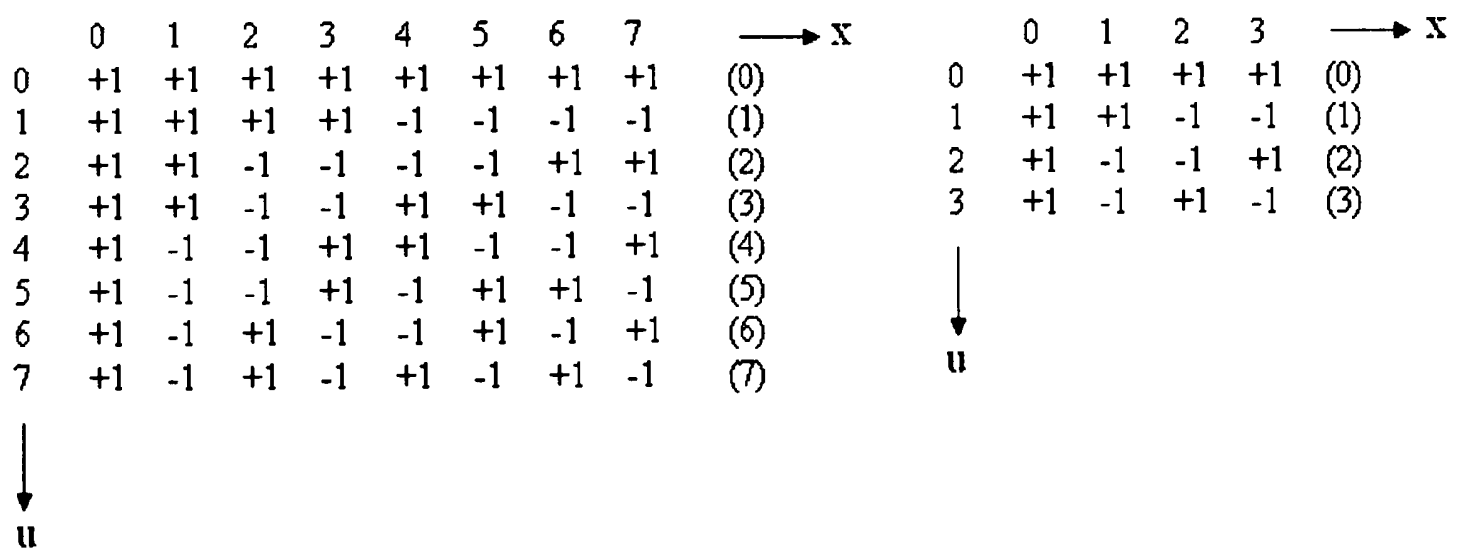
(a) $8 \times 8$ Walsh Matrix (Kernel)
(b) $4 \times 4$ Walsh Matrix (Kernel)

$\frac{1}{\sqrt{N}}(N=8$ or $N=4$ in these cases $)$ can be used with each kernel to yield an orthonormal basis. These $( \pm 1)$ elements of the Walsh transform can be generated for any $(n \times n)$ size 
matrix by using the relation $W(x, u)=\prod_{i=0}^{n-1}(-1)^{b_{i}(x) b_{n-1-i}(u)}$, where $b_{i}(k)$ is the $i^{\text {th }}$ binary bit of $\mathrm{k}$, and the matrix can be ordered with ascending sequency.

For the ordered Walsh kernel matrix, the Walsh operator $\omega_{N}^{r}$ of $r^{\text {th }}$ order and length $N$ is defined based on the sequency value (number of sign changes in its \pm l elements) and the dimension $N$ considered. The order $r$ is given by the sequency of the operator, and refers to the type of differences (derivatives) used between sample points. The dimension $N(N=2 n)$ refers to the width of the operator, function of the number of points considered. Considering the digitized input signal, $f(t)$, the Walsh transform defined by $W_{N}^{r}$ is given by the convolution $(*)$ of $\omega_{N}^{r}$ with $f(t)$ as:

$$
W_{N}^{r}=\omega_{N}^{r} * f(t)
$$

The Walsh operator of $1^{\text {st }}$ order and length $2, \omega_{2}^{1}$, is functionally equal to the $1^{\text {st }}$ derivative, $d^{1}$ :

$$
\omega_{2}^{1}=[1-1]=d^{1}=\frac{\partial}{\partial x}
$$

The Walsh operator of $2^{\text {nd }}$ order and length $4, \omega_{4}^{2}$, is functionally equivalent to the $2^{\text {nd }}$ derivative, $d^{2}$ :

$$
\omega_{4}^{2}=\left[\begin{array}{llll}
1 & -1 & -1 & 1
\end{array}\right] \cong d^{2}=\frac{\partial^{2}}{\partial x^{2}}=\left[\begin{array}{lll}
1 & -2 & 1
\end{array}\right]
$$


The results of the algorithm display automatically the detected spikes to facilitate manual observation. The mathematical steps considered in the development steps of the proposed algorithm are as follows:

\section{Implementation Steps of the Algorithm}

- After Walsh transforming each EEG channel, and in order to enhance identification of the sharp and steep transitions of potential spikes, the Walsh results at different scales (resolutions of $\mathrm{N}=4,8$, and 16) are added together to detect all potential transitions under different scaling (as revealed through higher amplitudes), expressed mathematically as:

$$
W_{4}^{r}+W_{8}^{r}+W_{16}^{r} \text { for } \mathrm{r}=1,2
$$

- Following these two graphs (for $r=1$ and $r=2$ ), and since the interictal spike must exhibit high degrees of sharpness in narrow and wider intervals, the resulting measures of sharpness/steepness in these intervals can be extracted through the functional equivalence of performing first and second order derivatives on the results obtained in the previous step. This is achieved with a point-by-point multiplication between the equivalent first and second order Walsh operators and the addition of the Walsh operators of different lengths $N$, given by $W_{4}^{r}+W_{8}^{r}+W_{16}^{r}(r=1,2)$. In other words the following operations are performed in this step to emphasize that part of the signal that meets sustained steep slopes and sharp peak: 


$$
\begin{aligned}
& \mathrm{W} 1=\omega_{2}^{r} \cdot W^{r}=\omega_{2}^{r} \cdot\left[W_{4}^{r}+W_{8}^{r}+W_{16}^{r}\right] \text { when } r=1 \quad \text {, and } \\
& \mathrm{W} 2=\omega_{2+r}^{r} \cdot W^{r}=\omega_{2+r}^{r} \cdot\left[W_{4}^{r}+W_{8}^{r}+W_{16}^{r}\right] \text { when } r=2
\end{aligned}
$$

Note in the results of this step that potential spikes will be predominant with the respect to the background.

- Dynamic thresholding is assumed next yielding $\mathrm{W}_{\mathrm{T} 1}$ and $\mathrm{W}_{\mathrm{T} 2}$ as thresholded version of W1 and W2 from the previous step. In order to extract that part of the signal deemed important from background activity, the threshold $(\mathrm{T})$ was computed as one standard deviation above the mean of all the peaks found in $3 \mathrm{~s}$ windows.

- $\quad$ The next step yields the signals denoted by $\mathrm{W}_{\mathrm{T1}}{ }^{*}$ and $\mathrm{W}_{\mathrm{T} 2}{ }^{*}$ containing only those components of the signals in $\mathrm{W}_{\mathrm{T} 1}$ and $\mathrm{W}_{\mathrm{T} 2}$ that satisfy all the established criteria as described in Section 2.3.2 of chapter 2. Note the two peaks in $\mathrm{W}_{\mathrm{T} 1}{ }^{*}$ which are indicative of the steepness of the rising and falling slopes of the spike, and the one prominent peak in $\mathrm{W}_{\mathrm{T} 2}{ }^{*}$ which accurately depicts the spike's peak location, and whose amplitude is related to the sharpness of the spike.

Figures 3.1 and 3.2 provide comparative results in the functionality of the Walsh operators versus those of the common mathematical derivatives. Figure 3.3 assesses the error signals in the functionality difference between the Walsh operators and their respective derivative operators.

These functional equivalencies are mentioned here to show that Walsh operators just like derivatives can indeed be used to extract sharp transitions and pinpoint through 
zero-crossings the location of a given peak, but yet, through orthogonality (independence between these operators), we can simultaneously use them in a unique way to extract specific features as those outlined for a spike.

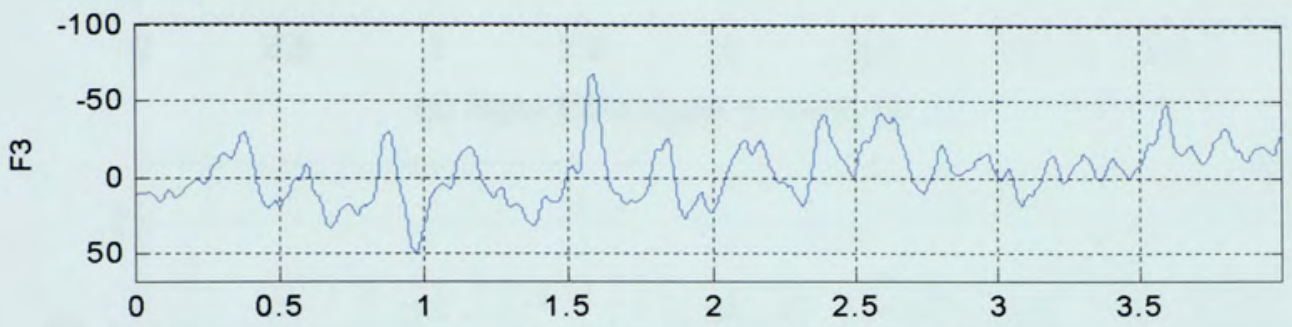

(a) Input EEG signal at electrode $\mathrm{F} 3$

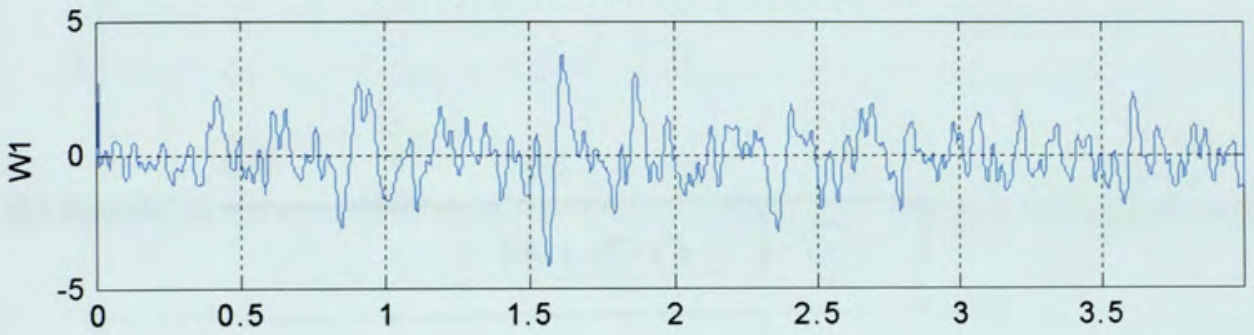

(b) Results of the convolution of the first-order Walsh operator with signal in (a):

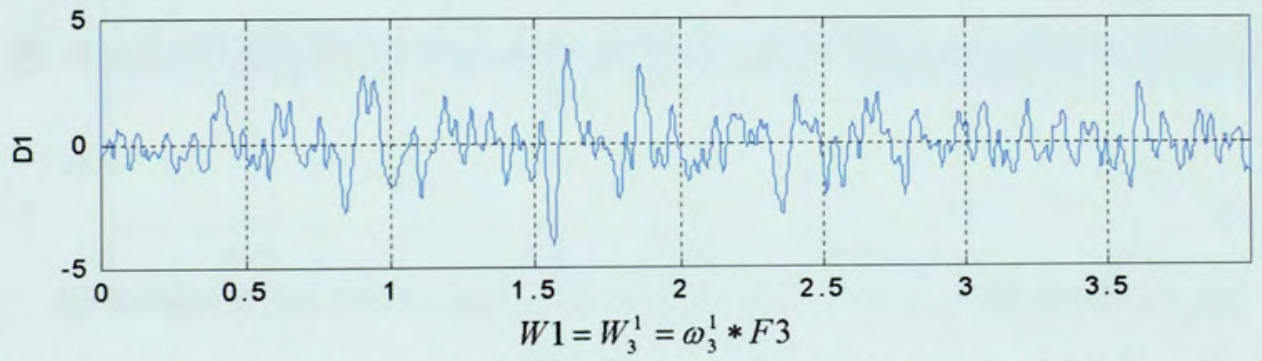

(c) Results of the convolution of the first-order derivative with signal in (a): $D 1=D^{1}=d^{1} * F 3$

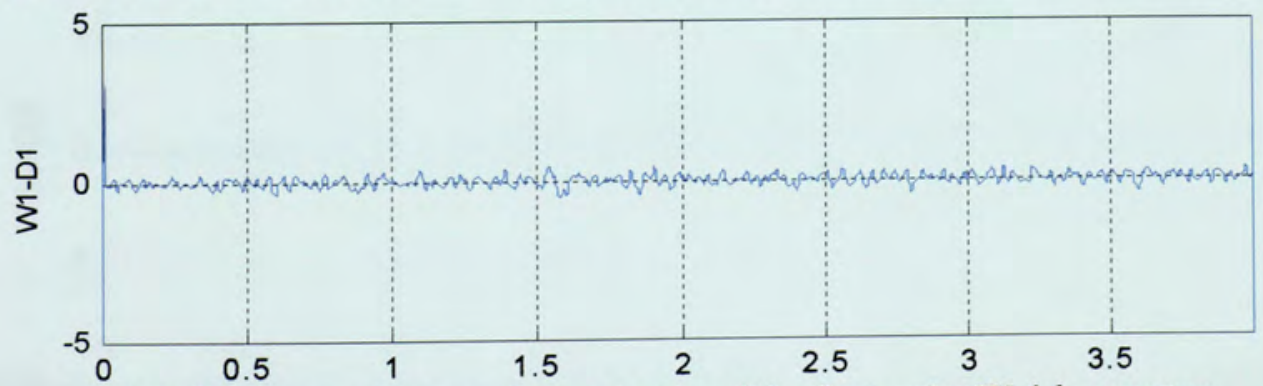

(c) Difference in the convolution results of the first-order Walsh operator versus the first-order derivative: $W 1-D 1=W_{3}^{1}-D^{1}$

Figure 3.1. Contrasting the functionalities of the first-order Walsh operator and the firstorder derivative. 


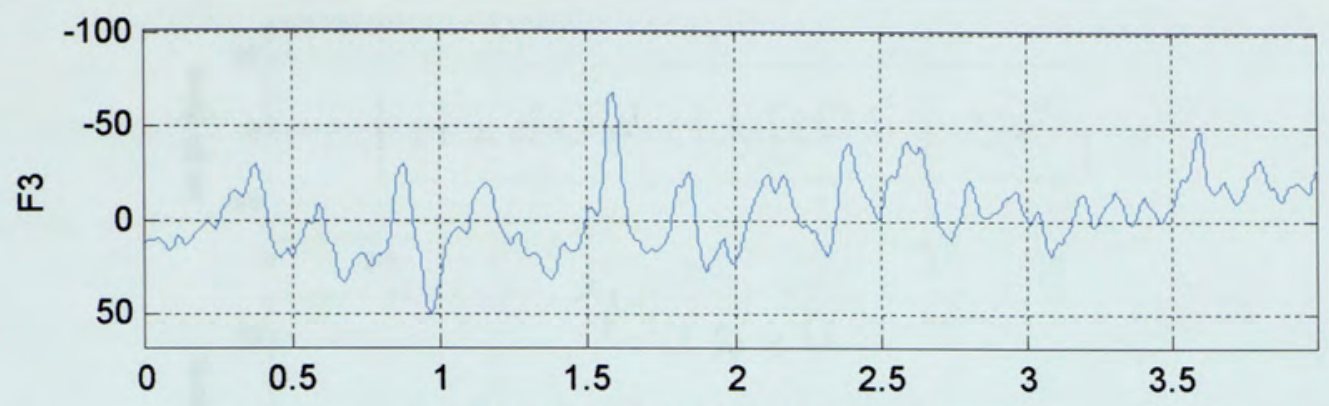

(a) Input EEG signal at electrode $\mathrm{F} 3$

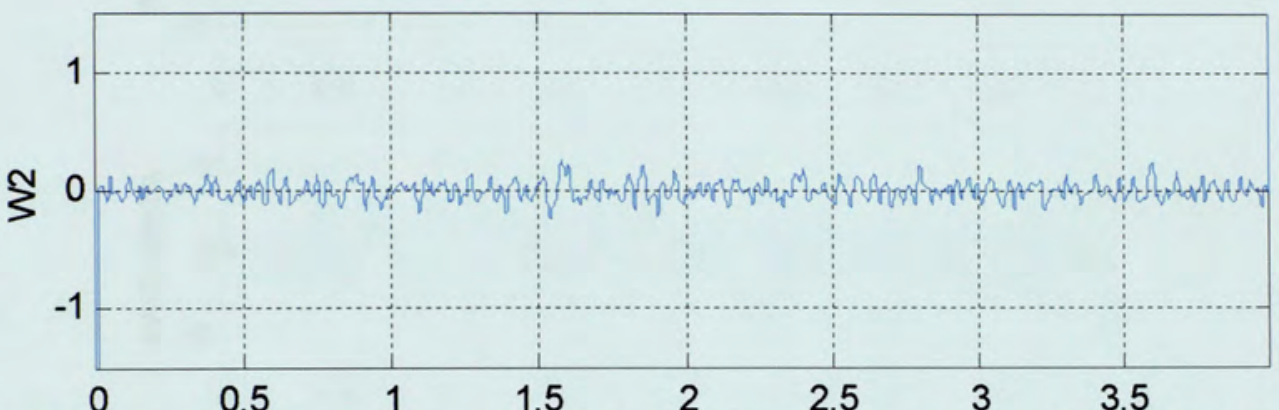

(b) Results of the convolution of the second-order Walsh operator with signal in (a):

$$
W 2=W_{3}^{2}=\omega_{3}^{2} * F 3
$$

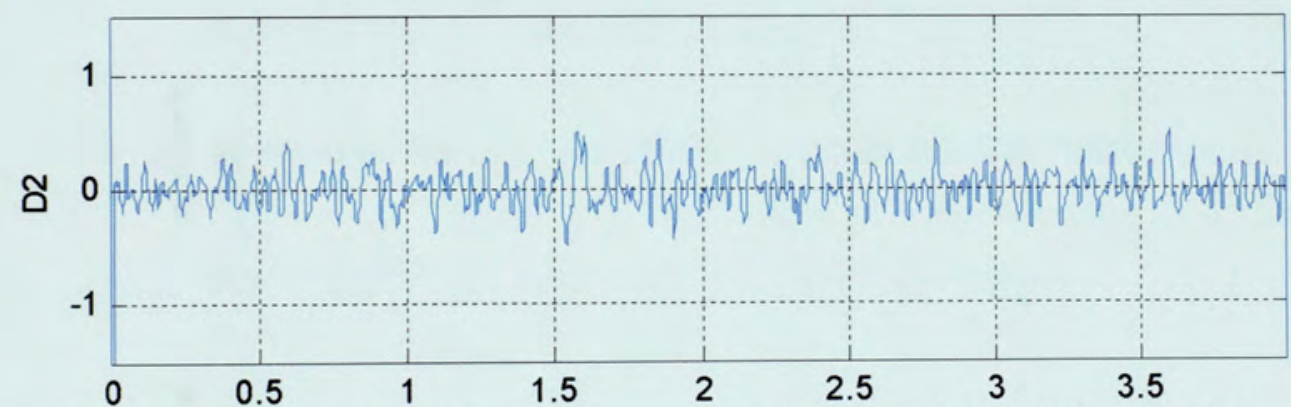

(c) Results of the convolution of the second-order derivative with signal in (a):

$$
D 2=D^{2}=d^{2} * F 3
$$

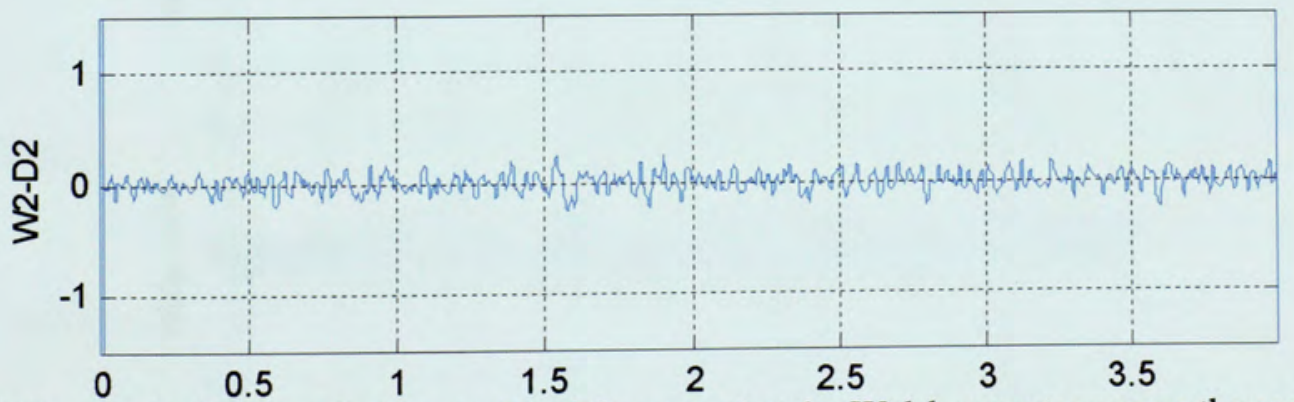

(c) Difference in the convolution results of the second-order Walsh operator versus the secondorder derivative: $W 2-D 2=W_{3}^{2}-D^{2}$

Figure 3.2. Contrasting the functionalities of the second-order Walsh operator and the second-order derivative. 

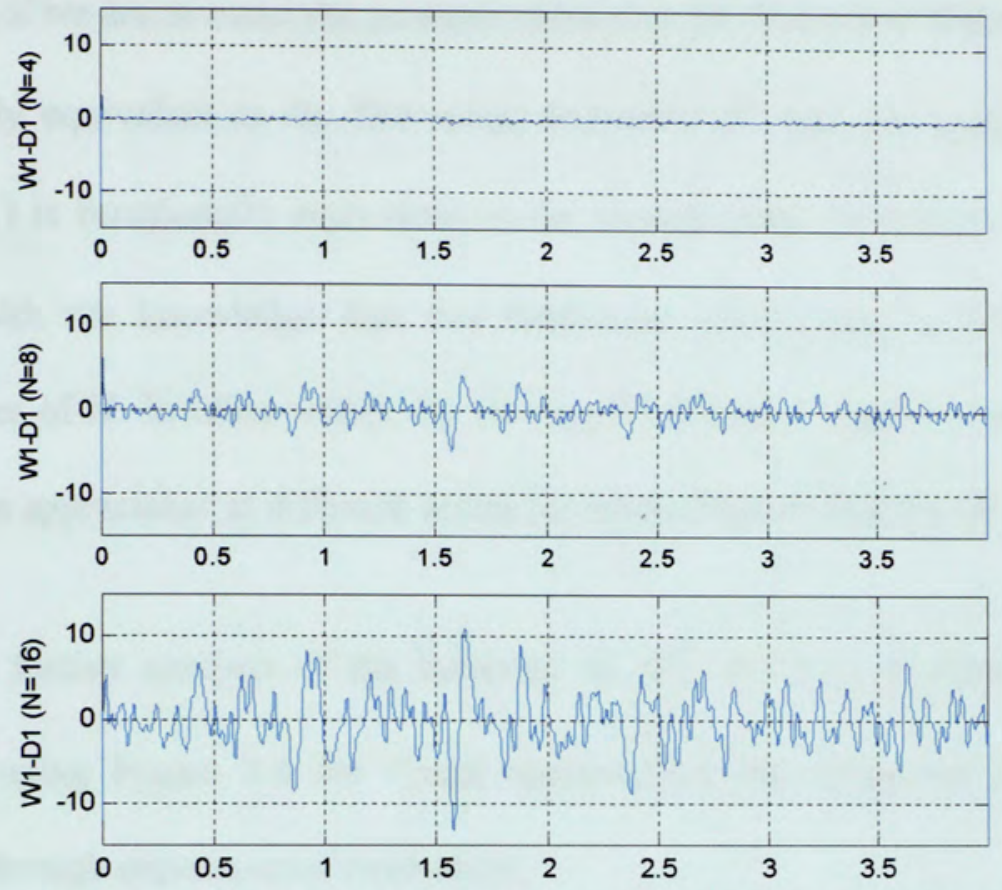

(a) Contrasting the differences in the convolution results of the first-order Walsh operator versus the first-order derivative: $W_{N}^{1}-D^{2}$ with $N=4,8$, and 16
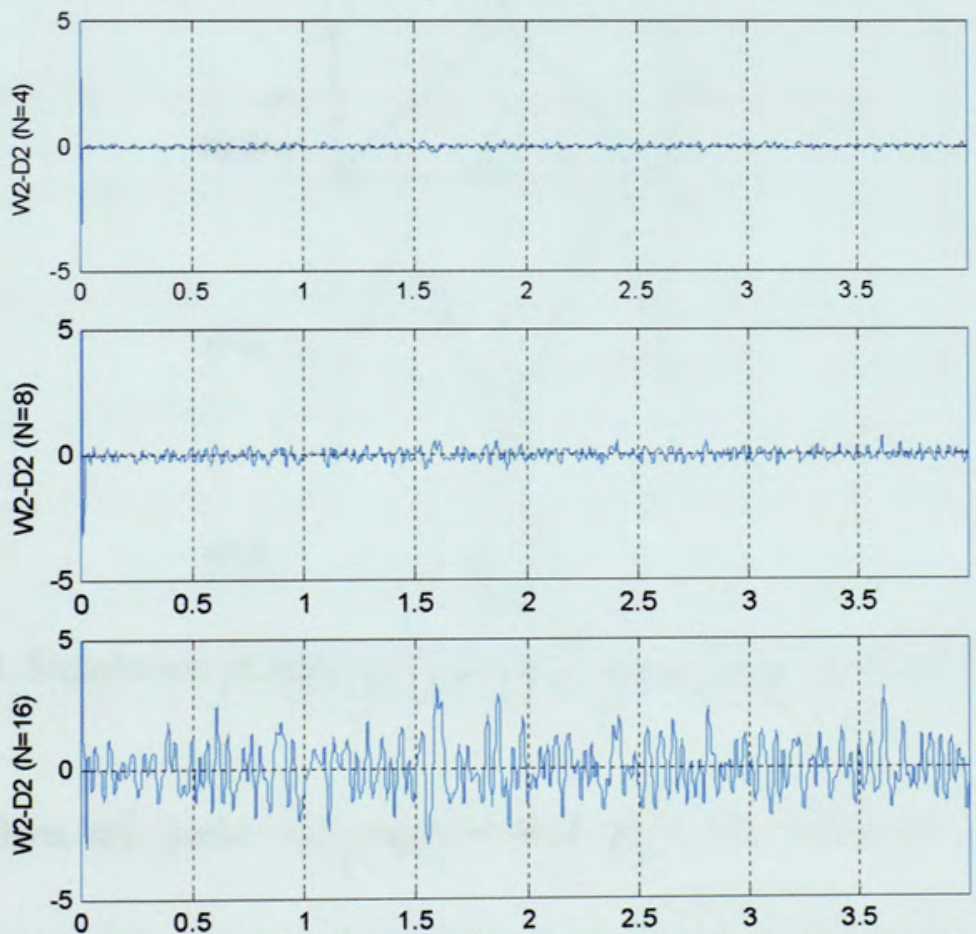

(b) Contrasting the differences in the convolution results of the second-order Walsh operator versus the second-order derivative: $W_{N}^{2}-D^{2}$ with $N=4,8$, and 16

Figure 3.3. Comparative results contrasting the errors (by difference) in functionality between the Walsh operators and their analogous mathematical derivatives. 
Thus, if we are to make the generalization that the first-order Walsh operator $\left(\omega_{N}^{1}\right)$ is functionally equivalent to the first-order derivative $d^{1}$, and the second-order Walsh operator $\left(\omega_{N}^{2}\right)$ is functionally equivalent to the second-order derivative $d^{2}$ for any $N$, it should be with the knowledge that this functional equivalence is more accurate for smaller values of $N$. In other words, by varying $N$, different characteristics of the input signal may be appreciated at different scales (or resolutions or degrees of fuzziness).

After further analysis of the behavior of $W_{N}^{r}(r=1,2)$ in relation to interictal spikes, and using Figure 3.4 for visual appreciation, the following set of rules are established through experimental evaluation:

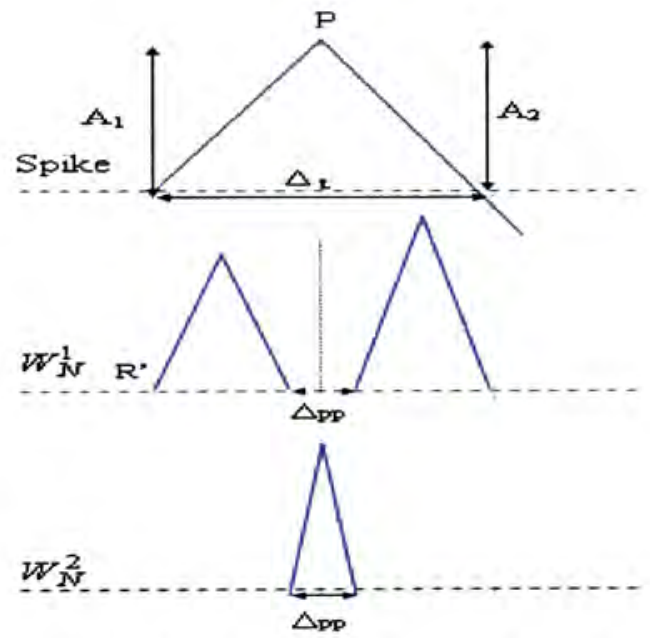

Figure 3.4. Simulation of interictal spike with anticipated results in $W_{N}^{1}$ and $W_{N}^{2}$.

- The results from $W_{N}^{1}$ yield two peaks for each spike. The first peak is associated with the rising slope, and the second with the falling slope. The amplitude of each peak in $W_{N}^{1}$ is an indicator of the steepness of the slope, where a higher peak means a steeper slope. 
- The results from $W_{N}^{2}$ yield a peak associated to the spike's peak location. The amplitude of this peak in $W_{N}^{2}$ relates to the sharpness of the corresponding spike, where a higher peak means a sharper spike.

\subsubsection{THE IMPLEMENTATION STEPS}

The following steps are presented in the same sequence that they are implemented in the algorithm described in the Appendix. Intermediate results for each step are revealed to assess both the validity of such steps and the merit of each step for identifying interictal spikes and for rejecting artifacts.

\section{Step 1- Satisfying Criterion 1: Sharpness of a spike is continuous}

Continuity in sharpness in a spike means that it is sharp in narrow as well as in wider intervals of observation. This implies that high values must result for the peaks in $W_{N}^{1}$ and $W_{N}^{2}$, with $N=4,8$, and 16 used as the respective number of points analyzed simultaneously in the input data. For wider intervals of observation, the algorithm developed takes the results at different scales (resolutions) and adds them together to detect all potential transitions under different scaling. In other words, sharpness/steepness of the signal identified in any of the $W_{4}^{r}, W_{8}^{r}$ and $W_{16}^{r},(r=1,2)$ signals, resulting in highamplitude peaks, should yield a steep and sharp signal in $W^{r}$.

Furthermore, interictal spikes must also exhibit high local sharpness and steepness. The best way to measure this observation is through convolutions with the first 
and second order Walsh operators, which will provide a high peak and a zero crossing, emphasizing and localizing that part of the signal that meets sustained steep slopes and sharp peak.

\section{Step 2-Satisfying Criterion 2: Rising and falling slopes of the spike are both steep}

To ensure that the rising and falling slopes of the spike are both very steep, we consider the two peaks resulting in $W^{1}$ and the one peak resulting in $W^{2}$ in direct association with the steep slopes of the spike. A maximum duration of $20 \mathrm{~ms}$ for the "valley" created between these two peaks, defined as $\Delta_{\mathrm{PP}}$ confirms the steepness of the slopes. The $20 \mathrm{~ms}$ condition is obtained empirically through the so-called training set used in the study. In this implementation step, if the separation $\Delta_{\mathrm{PP}}$ is greater than that set duration, then the first peak is eliminated and the check is made again for the second peak, and so on. The result of this procedure is that $W^{1}$ would contain only peaks that are related to signals that have both a steep rising slope and a steep falling slope.

\section{Step 3- Satisfying Criterion 3: A sharp peak characterizes the spike}

The criterion that a sharp peak $P$ characterizes the spike is resolved through set dynamic thresholds both in the temporal and spatial domains, and through verification in the peak resulting in $\mathrm{W}^{2}$. This implementation step yields the following observations:

- Dynamic thresholds in temporal and spatial domains are found effective given the variations in the signal's background activity. With the choice of windows of $3 \mathrm{~s}$ 
intervals, these thresholds are set at one standard deviation about the mean of all the peaks found in these $3 \mathrm{~s}$ windows.

- $\quad$ The amplitude of the peak in $W^{2}$ is proportional to the sharpness of the peak in the input signal that has satisfied the threshold conditions. This peak in $W^{2}$ occurs in an interval corresponding to $\Delta_{\mathrm{PP}}$ in $W^{1}$.

It is noted that Walsh signals are reviewed to evaluate spatial sharpness with regard to both the order of complexity (that is $r=1,2$ ) and the different degrees of fuzziness $(N=4,8$, and 16 , which is the size of the Walsh operator used).

\section{Step 4- Satisfying Criteria 4 - 7: Characterizing spike's duration, half waves and}

\section{amplitude}

Since criteria 4, 5, and 6 are based on conditions already established using specific measured bounds, this implementation step was to assess and potentially modify such measured bounds through empirical observation using the training set consisting of the 10 patients. In fact, we consider criteria 4,5 , and 6 to be added measures of certainty to the detection of spikes beyond satisfying criteria 1,2 , and 3 .

In this implementation step, the following changes were considered in the final algorithm:

- If we consider the fact that a sharp transient has a total duration between 70 to 200 $\mathrm{ms}$, the total duration of an interictal spike can then extend anywhere between 20 to 200 
ms. The training set using the 10 patients revealed that a change of range in this duration from 50 to $215 \mathrm{~ms}$ would be more appropriate to minimize artifactual activity on the lower bound and to incorporate wider spikes as detected by medical experts on the higher bound. This was a sensible compromise between missing out on artifacts and the possibility of missing out on detecting narrow spikes, since the automated process for artifact removal was consistently successful with the integrated algorithm.

- The ratio of amplitude between a potential spike and the background activity can be made to be greater than 1.5 (e.g. 1.6) in order to overcome the potential effects of EKG data, based on empirical observation of the training set. We however opted for eliminating EKG artifacts based on their periodicity, rather than subjectively increasing this aspect ratio.

Step 5- Satisfying Criteria 8 - 10: Multi-channel activity, spike-like transients, and slow wave following a spike

Criteria 8 and 10 are defined as support observations used to increase the certainty in identifying a spike but not to undermine it. Criterion 9 is established to ensure that the effects of artifactual data are either identified as such or filtered out to yield a minimized false detection rate.

It is noted however that prior to determining whether the established criteria are satisfied or not, the first performed preprocessing step is that of filtering out or reducing background signal from potential events. In order to eliminate the background signal, we compared the heights of the peaks in each of $W^{1}$ and $W^{2}$ within a window of 3 seconds 
and if the heights of the peaks in that time range were below the computed threshold which is set as one standard deviation about the mean of all the peaks found in these $3 \mathrm{~s}$ windows, then such behavior is associated with the background signal, and thus eliminated from further consideration in the transformed signals.

The purpose of criteria 8 (multi-channel activity), especially when dealing with neighboring electrodes with close proximity, is to simply increase the validity in assuming that a potential spike is indeed an actual spike based on the fact that spikes do not occur in isolation, although criteria (1 through 7 ) established in the algorithm are already stringent in identifying a spike in any one channel. Of course inter-electrode spacing of the recording system used should be considered in defining neighboring channels; and the proximity of these channels is important in the case where such electrode spacing is necessary for accurate 3-D localization of epileptic interictal spikes (Mirkovic et al., 2003).

Criteria 9 involve spike-like transients (artifactual data) which were dealt with automatically through cautionary measures integrated into the algorithm. Implementation details for identifying or eliminating the effects of eye blinks, EKG and EMG are provided in Section 3.2

The purpose of criteria 10 (slow wave following spike) is also to increase the certainty of identifying an actual spike (a true positive): a slow wave is identified in the $W^{1}$ signal, where two peaks follow the peaks associated with a spike. These peaks correspond to the rising and falling slopes of the slow wave whose duration is estimated 
between 100 and $200 \mathrm{~ms}$. In addition, they are separated with a "valley" corresponding to the small slope (close to zero) of the slow wave.

It is important to note that all of these checks in all these steps were performed through the use of the $W^{r}$ signals (for $r=1,2$ ), as opposed to using the original EEG signal itself. Such a fact, which exploits the orthogonality property, will be demonstrated through the results obtained.

\subsection{RESULTS}

The spike detection algorithm was run on epileptogenic EEG using the testing set (10 of the 31 subjects). The "gold standard" is: identification of spikes by both medical advisors scoring the files. The following terms are thus defined:

- TRUE POSITIVE (TP): The algorithm and at least one of the medical advisors declare the presence of a spike.

- FALSE POSITIVE (FP): The algorithm identifies a spike that none of the medical advisors did.

- FALSE NEGATIVE $(F N)$ : at least one medical advisor identifies a spike that the algorithm did not.

The standard measures of sensitivity and precision (Eberhart et al., 1990) are used for the evaluation of the algorithm:

- Sensitivity $(S)$ is " the likelihood that an event will be detected given that it is present", defined as:

$$
S=T P /(T P+F N)
$$


- Precision (P) or positive predictive value is "the likelihood that a signal of an event is associated with the event, given that a signal occurred" [21], defined as:

$$
P=T P /(T P+F P)
$$

\subsubsection{IMPLEMENTATION RESULTS AND ANALYSIS}

The results of the algorithm display automatically the detected spikes to facilitate manual observation. These results are given in Tables 3.1 and 3.2. The sensitivity and precision results are illustrated in Tables 3.3 and 3.4.

Table 3.1. Results for both training and testing sets for spike identification.

Note: Spikes are annotated by two experts.

\begin{tabular}{|c|c|c|c|c|c|c|c|}
\hline Set & NS & TP & FP & FN & $\mathbf{S}_{1}$ & $\mathbf{S}_{2}$ & $\mathbf{S}_{\mathrm{B}}$ \\
\hline $\begin{array}{c}\text { Training } \\
\text { (10 patients) }\end{array}$ & 51 & 46 & 1 & 5 & 19 & 20 & 19 \\
\hline $\begin{array}{c}\text { Testing } \\
\text { (21 Patients) }\end{array}$ & 186 & 148 & 23 & 38 & 100 & 89 & 58 \\
\hline $\begin{array}{c}\text { Both Training and } \\
\text { Testing }\end{array}$ & 237 & 194 & 24 & 43 & 119 & 109 & 78 \\
\hline
\end{tabular}

Table 3.2. Results for both training and testing sets for spike identification. Note: Spikes are annotated by three experts.

\begin{tabular}{|c|c|c|c|c|c|c|c|c|}
\hline Set & NS & TP & FP & FN & $\mathbf{S}_{1}$ & $\mathbf{S}_{\mathbf{2}}$ & $\mathbf{S}_{\mathbf{3}}$ & $\mathbf{S}_{\mathbf{t}}$ \\
\hline $\begin{array}{c}\text { Training } \\
\text { (10 patients) }\end{array}$ & 51 & 46 & 1 & 5 & 19 & 20 & - & - \\
\hline $\begin{array}{c}\text { Testing } \\
\text { (21 Patients) }\end{array}$ & 319 & 261 & 23 & 58 & 100 & 89 & 133 & 58 \\
\hline $\begin{array}{c}\text { Both Training } \\
\text { and Testing }\end{array}$ & 370 & 307 & 24 & 63 & 119 & 109 & 133 & 58 \\
\hline
\end{tabular}

Note in Tables 3.1 and 3.2 the NS $=$ total number of spikes identified. $S_{1}, S_{2}$, and $S_{3}=$ spikes identified, by $1^{\text {st }}, 2^{\text {nd }}$, and $3^{\text {rd }}$ EEG expert, respectively. Note that NS= 
TP+FN. Note also that $(-)$ in the table means that spikes identified by the third expert were not used in the training phase to stay consistent with Table 3.1. Only the testing phase results were important in this case.

Table 3.3. Sensitivity $(S)$ and precision $(P)$ measurements for the phases of training and testing when two experts annotated the spikes.

Note: Recall that $S=T P /(T P+F N)$ and $P=T P /(T P+F P)$.

\begin{tabular}{|c|c|c|}
\hline Set & Sensitivity & Precision \\
\hline Training & 0.90 & 0.98 \\
\hline Testing & 0.80 & 0.87 \\
\hline Both & 0.82 & 0.89 \\
\hline
\end{tabular}

Table 3.4. Sensitivity $(S)$ and precision $(P)$ measurements for the phases of training and testing when three experts annotated the spikes.

Note: Recall that $S=T P /(T P+F N)$ and $P=T P /(T P+F P)$.

\begin{tabular}{|c|c|c|}
\hline Set & Sensitivity & Precision \\
\hline Training & 0.90 & 0.98 \\
\hline Testing & 0.82 & 0.92 \\
\hline Both & 0.83 & 0.92 \\
\hline
\end{tabular}

Figures 3.5-3.8 provide different results on different patients illustrating interesting detection outcomes. In these figures, it should be noted that the actual size consists of $1 \mathrm{sec} / \mathrm{div}, 30 \mathrm{~mm} / \mathrm{sec}$ with a Gain of $30 \mathrm{uV} / \mathrm{mm}, \mathrm{LFF}=1 \mathrm{~Hz}, \mathrm{HFF}=70 \mathrm{~Hz}$, and Notch of $60 \mathrm{~Hz}$. In the presentation of these results, for each patient, an EEG record is first provided where the algorithm has identified automatically a TP, a FP or a FN. This EEG record is then followed by the intermediate steps as outlined in the algorithm provided before, and as portrayed in the examples of the results obtained in Figures 3.53.8 for interictal spike detection and in Figures 3.10-3.12 for artifact identification and removal. Figure 3.9 illustrates an example where multi-channel activity was detected. 

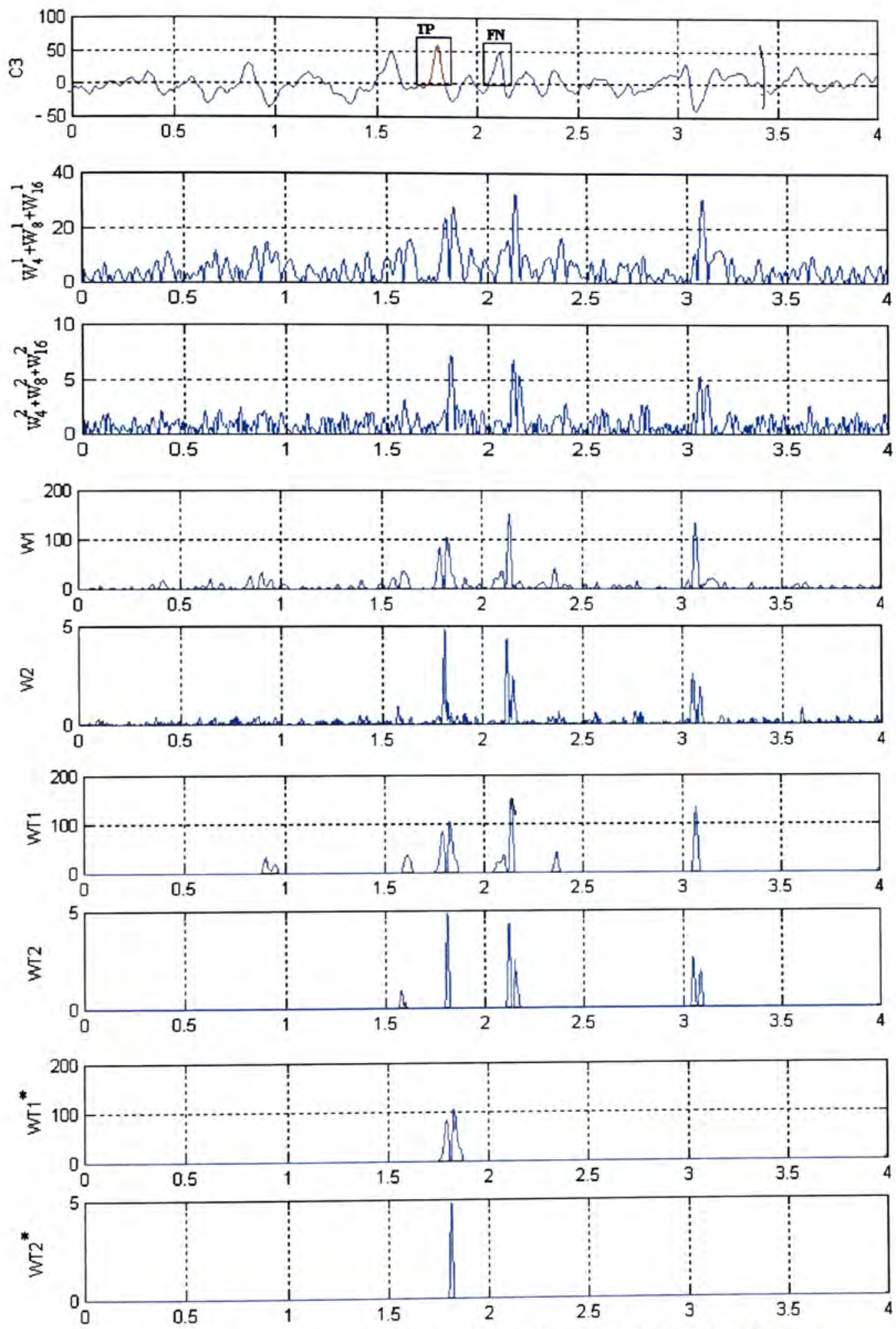

Figure 3.5. Examples of results illustrating the detection of spikes for patient 15 in channels $\mathrm{C} 3$. 



Figure 3.6. Examples of results illustrating the detection of spikes for patient 5 in channels F3. 




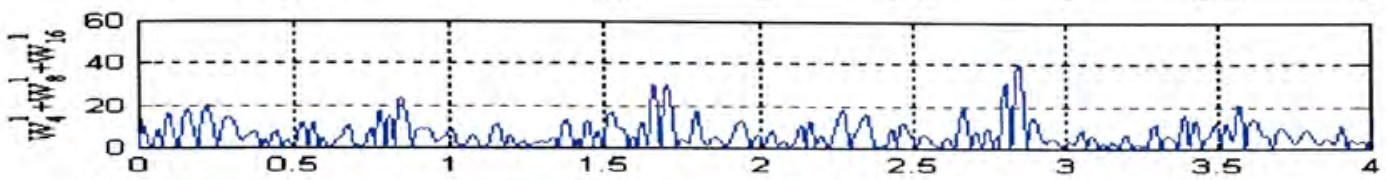

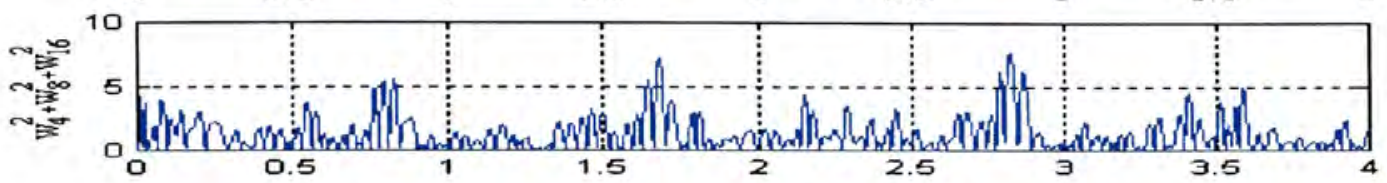
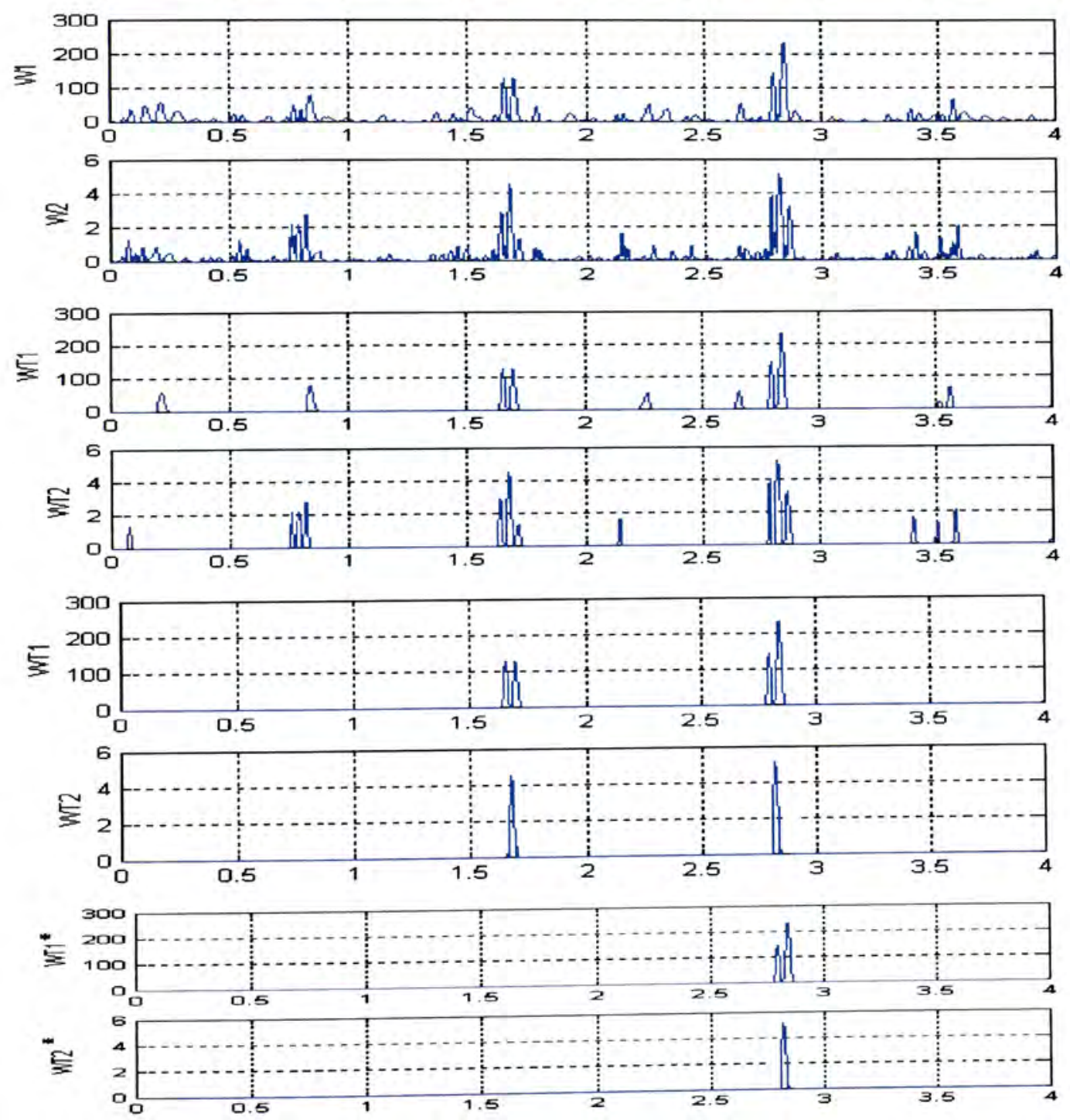

Figure 3.7. Examples of results illustrating the detection of spikes for patient 3 in channels FP2. 


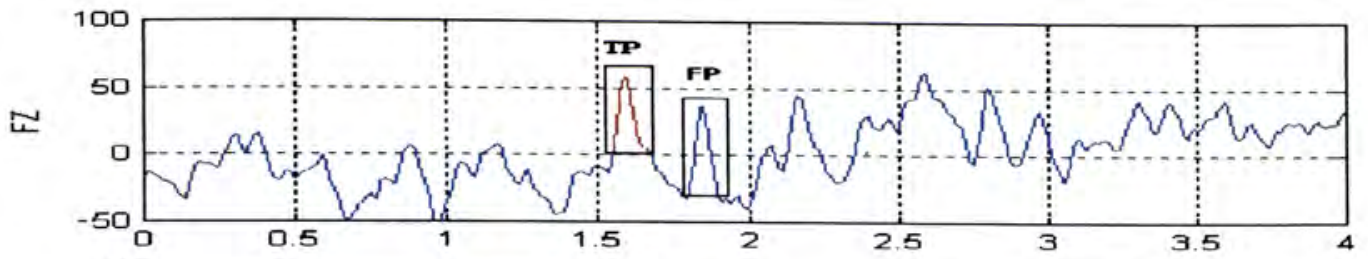

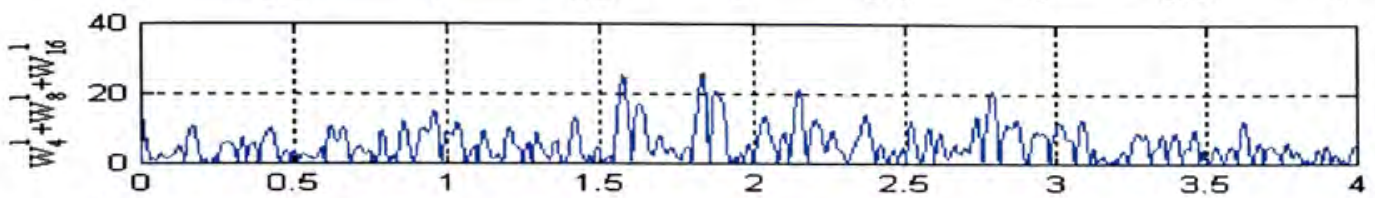
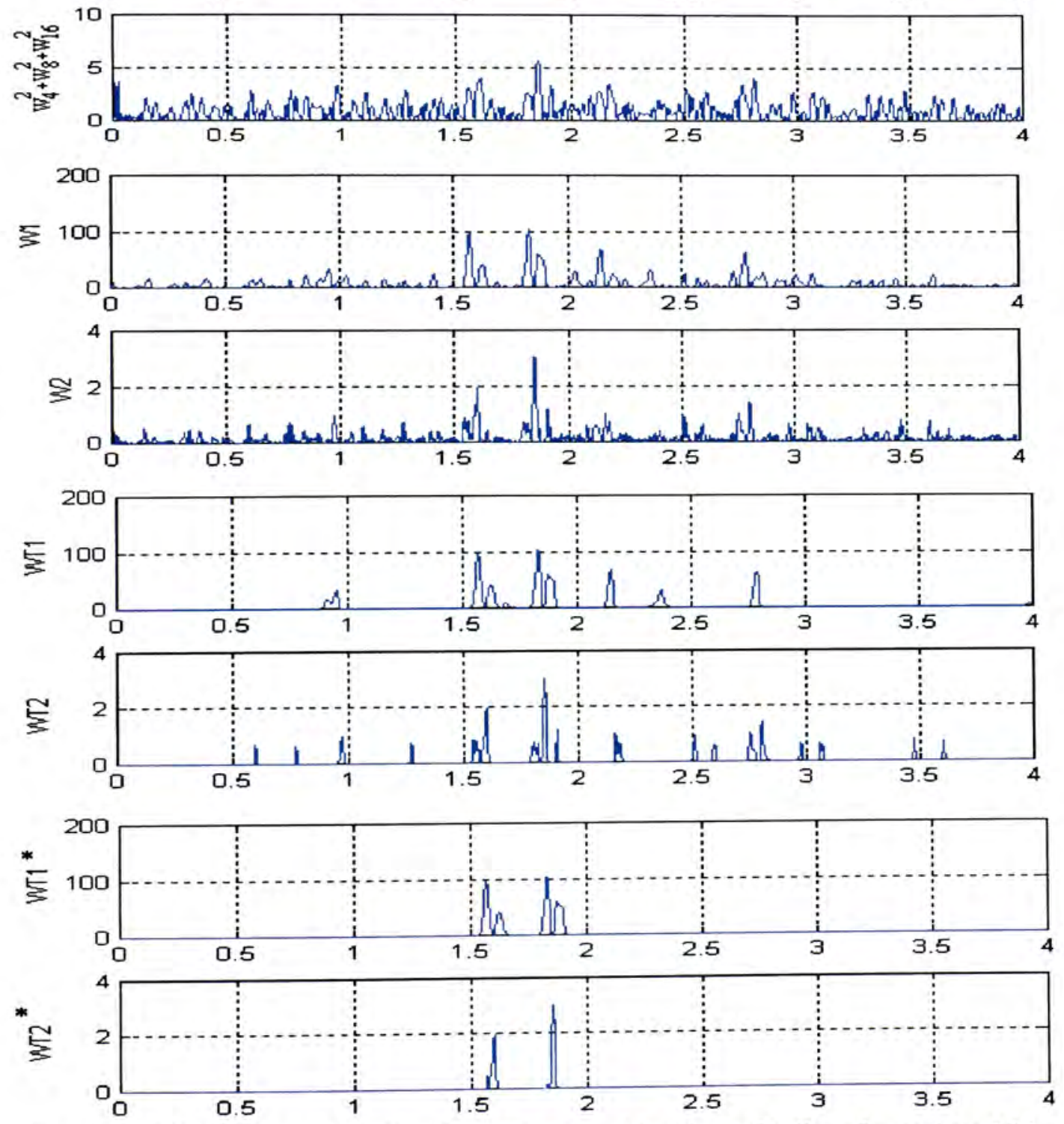

Figure 3.8. Examples of results illustrating the detection of spikes for patient 10 in channels FZ. 


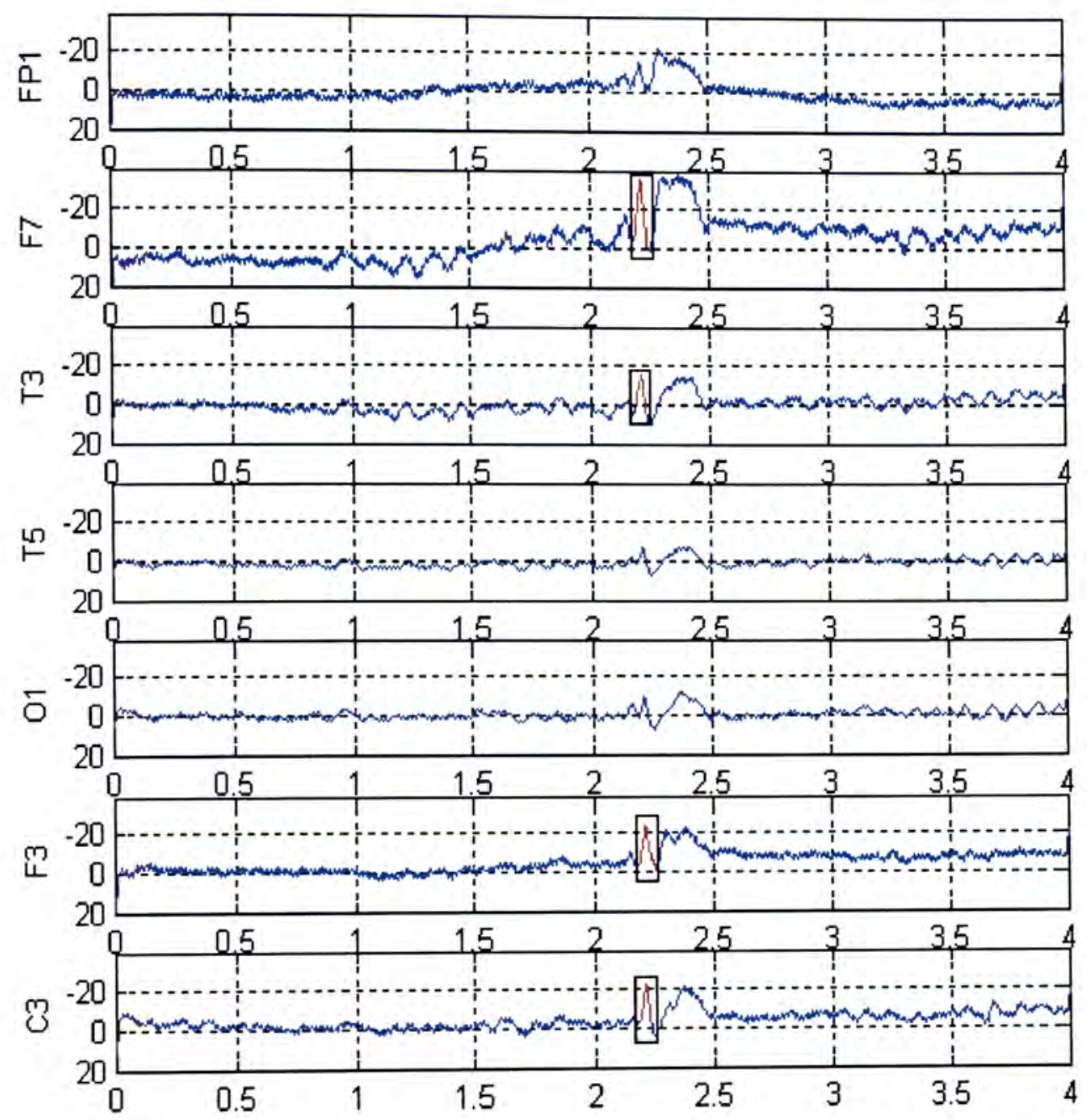

Figure 3.9. Results showing multi-channel activity with detected spikes in channels F7, T3, F3 and C3.

\subsubsection{RESOLVING CONTENTIOUS ISSUES OF ARTIFACTUAL DATA}

The algorithm developed included in its implementation cases of spike-like transients (artifactual data) that needed to be identified as such automatically to minimize any false detection, which was accomplished in this case, as preprocessing steps for 
EKG, Eye blinks, and EMG data. Since subdural recordings were used in this dissertation, these artifacts are not an issue. But, in anticipation that such an approach could also be implemented using external recordings (at the scalp level) one has to content with them. What follows are strategies that were undertaken to alleviate or eliminate the effect of these artifacts on the spike detection.

For the removal of EKG artifacts, the periodicity of the EKG behavior was exploited. After applying the Walsh transform to the EEG signal and obtaining the $W^{1}$ and $W^{2}$ signals, and after filtering out the background signal they are checked for periodicity as follows: If five consecutive peaks satisfying the set thresholds in the $W^{1}$ and/or $W^{2}$ signals are found within $500 \mathrm{~ms}$ to $1 \mathrm{~s}$ apart from each other, then those waves are classified as periodic and may be associated with EKG waves, and certainly not as potential spike waves. Note that with the choice of windows of $3 \mathrm{~s}$ intervals, the chosen thresholds are set at one standard deviation about the mean of all the peaks found in these $3 \mathrm{~s}$ windows. As an additional measure of caution, note also that the criteria defining a spike are violated in the thresholded $W^{1}$ and/or $W^{2}$ signals as shown in Figure 3.11.

For the artifacts of eye blinks and EMG, as illustrated in Figures 3.12 and 3.13, one or more of criteria $(1,2,3$, or 5$)$ were not satisfied through the analysis of W1 and W2 signals. Interesting results obtained when dealing with "Eye Blink" artifact showed reversed characteristics sought by the search criteria using the Walsh operators in W1 and W2 (with one peak in W1 instead of 2 and 2 peaks in W2 instead of 1). 

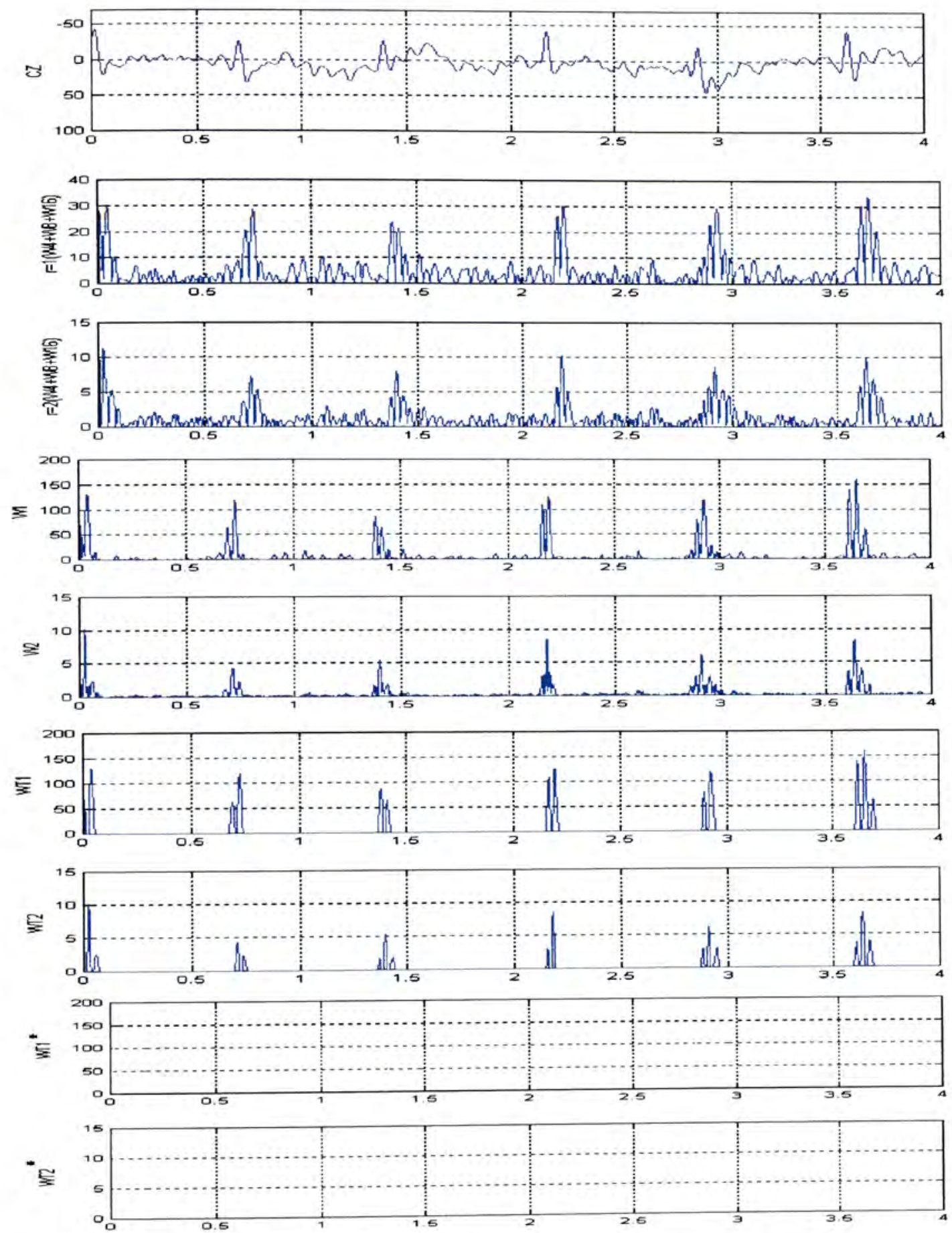

Figure 3.10. Results obtained when dealing with EKG data - note the periodicity in EKG and how the characteristics sought by the search criteria using the Walsh operators are not met in WT1 and WT2. 

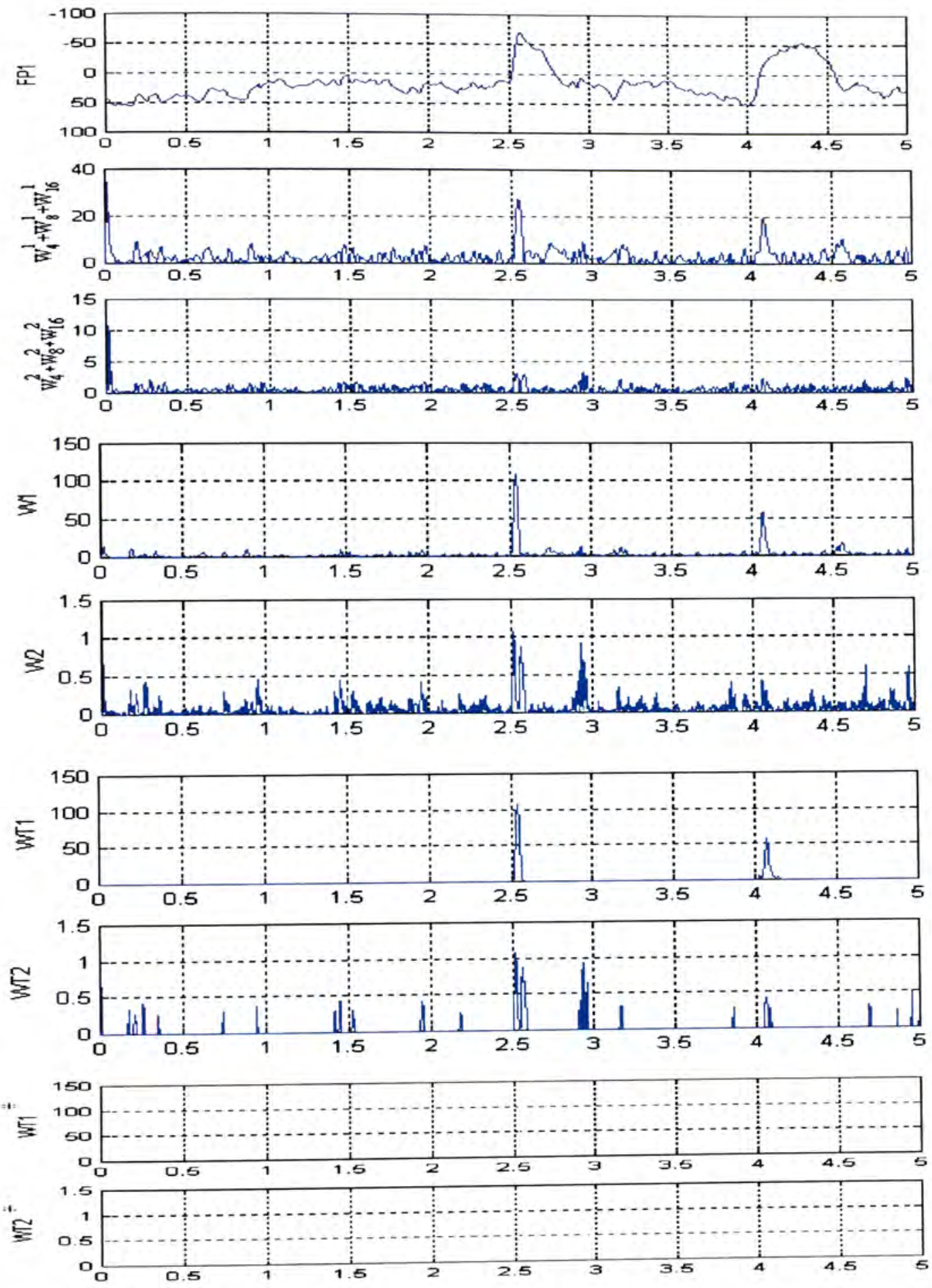

Figure 3.11. Results obtained when dealing with "Eye Blink" artifact - note how the characteristics sought in WT1 and WT2 are not met, and that WT1 and WT2 have a reversed behavior with respect to a spike (with one peak in W1 instead of 2 and 2 peaks in W2 instead of 1 ). 

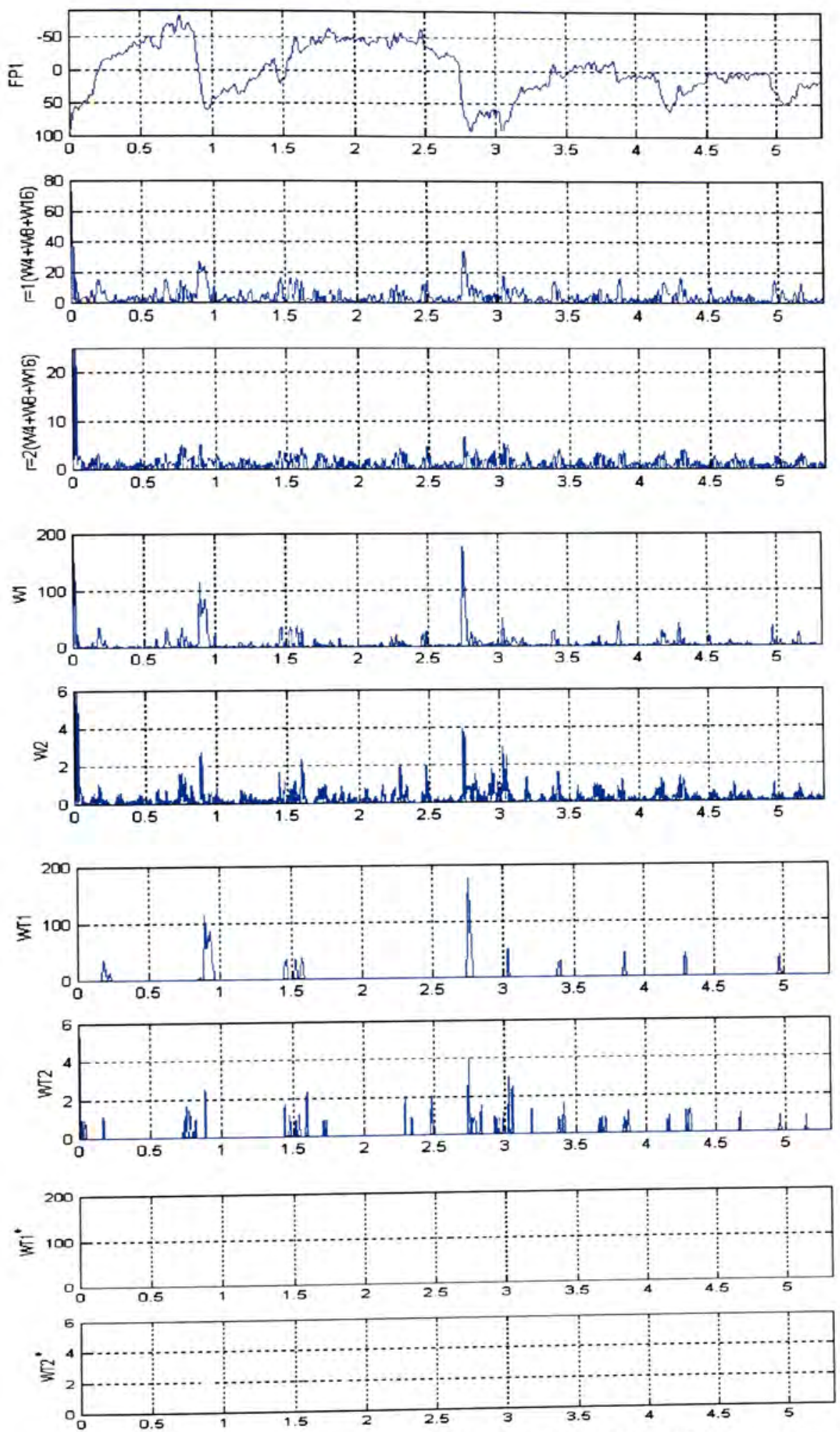

Figure 3.12. Results obtained when dealing with EMG artifact - note how the characteristics sought by the search criteria using the Walsh operators in WT1 and WT2 are not met. 


\subsubsection{DISCUSSION OF THE RESULTS}

In retrospect, the related studies we have reviewed including those included in the review article (Wilson and Emerson, 2002), and taking into consideration the use of existing algorithms such as: Gotman spike detector (GSD), two-stage spike detector (2SSD), multiple monotonic neural network (MMNN), wavelet spike detector (WSD), to name a few, show the following varied results:

Sensitivity: being the most commonly used performance metric varied between low values ranging between $15 \%$ to $52 \%$ and high values ranging between $70 \%$ and $97 \%$.

Selectivity, Precision, and Specificity: These metrics are used intermittently from study to study. We also determined that the performance metric Selectivity was used with different definitions: sometimes as the False Positive rate FP/(FP+TN), sometimes as the negative prediction value or Specificity $\mathrm{TN} /(\mathrm{TN}+\mathrm{FN})$ and still at other times as the positive prediction value or Precision TP/(TP+FP). The reported values ranged between $40 \%$ and $92 \%$. The high $92 \%$ was accomplished with a limited example data set of 73 events. In related studies where events were much higher (300 events or more), the sensitivity/selectivity varied between $70 \% / 65 \%$ to $80 \% / 40 \%$ which is more like the mean of what other studies report.

In all of these performance values, it can be said that the good results were accomplished when the number of patients was relatively small ranging from 2 to 18 , or when the number of events is small (less than 100). For example, for the case where the low values were obtained was for the specific study where the patient count was 50 . 
Studies involving higher number of patients (greater than 50) did not unfortunately provide these performance metrics.

It should be made clear that given the many different research aspects used in the related studies along with the variety and inconsistent use of metrics such as (precision, specificity, sensitivity, selectivity), number of patients used (or number of events considered), the lack of providing performance metrics in others, did not permit for a clear cut comparison and made it difficult to produce a fair assessment of all the results.

In this study we formulated and evaluated characterizing features of interictal spikes using orthogonal operators that were designed based on the Walsh transformation. We translated each of the observable characteristics into mathematical expressions such that each and every one of the characteristics is implemented in the development of our algorithm. The uniqueness of this algorithm is in the establishment of a mathematical foundation capable of extracting potential spikes from background EEG signal using mutually independent Walsh signals which served as an orthogonal basis for analysis.

When three experts annotated the spikes, and with the 31 subjects used, the results based on the test samples only (21 patients out of the 31 ) reveal a precision of $82 \%$ and a sensitivity of $92 \%$, with a false positive (FP) detection rate of about $1.8 \mathrm{FP}$ per hour of EEG recording, given a 20 to 30 - minute epochs of continuous EEG recording per subject. Given the complex nature of EEG recordings, these results, which are supported by clinical experimentation, are most encouraging. The integrated algorithm proposed is computationally efficient, fully automated, and integrated in a way 
that it uses only Walsh operators in a unique way for the extraction of interictal spikes. Artifacts due to EMG, EKG or eye blinks were discarded automatically by virtue of cautionary processing steps embedded in the algorithm.

The use of such an algorithm is foreseeable in automated techniques that combine EEG and other sensory modalities for 3-D localization of epileptic foci (Ebersole et al., 1993; Barkley and Baumgartner, 2003; Mirkovic et al., 2003). Further developments of the EEG analysis component of the algorithm can also extend into the scrutiny of the transitional aspects between interictal and ictal phases to integrate nonlinear mechanisms for anticipating seizures (Lehnart et al., 2001; Le Van Quyen, 2001, and Yaylali, 1996).

What is described in this chapter serves as a precursor to the next study, in view of our augmented understanding of such interictal EEG recordings. This leads us to a novel approach, which is viewed as the core of this dissertation. Such an approach shows great promise, as shall be described, in that it is integrated and operates effectively on interictal EEG signals through a neural-network-based classification process which delineates electrodes that lead to seizure from those that do not. 


\section{CHAPTER 4}

\section{PRELIMINARY EEG DATA MANIPULATION WITH THE PURPOSE OF FINDINGS KEY PARAMETERS TO ANALYZE THE SIGNALS}

\subsection{INTRODUCTION}

EEG signals have played an important role in the modeling of the brain's cortical dynamics and have been analyzed over the past decades with much effort towards a better understanding of the function of the brain.

In the past, researchers have considered different approaches using a diversity of linear and nonlinear parameters in order to automate seizure detection and prediction (Wilson, et. al., 2002). Some of these parameters implemented in this dissertation are briefly described below based on the general themes they are set to exploit.

\subsection{PARAMETERS IMPLEMENTED}

In this preliminary study, a variety of parameters were initially investigated, so that reliable measurements could be used to delineate between the two groups of electrodes (leading and not leading to seizure). The behavior of these parameters is assessed with respect to the electrodes that lead to seizure in red and in contrast to the electrodes that do not lead to seizure in blue, so a visual analysis could be performed. As can be observed, all of these measurements are very difficult to asses in terms of discriminating the two groups of electrodes. It is with the intent to solve such a problem that several parameters are thoroughly analyzed in this dissertation. 


\subsubsection{AUTOCORRELATION OF THE EGG}

The autocorrelation of the signal in time domain is performed using equation 4.1. The autocorrelation function can be used to detect the non-randomness in the EEG data and to identify an appropriate time series model if the EEG data is not random. If the EEG data is random, autocorrelation should be near zero for any and all time-lag separations. If non-random, then the autocorrelation will be significantly non-zero. The constant $\mathrm{k}$ used in this study was 10 . The following formula was used to calculate the autocorrelation values in time.

$$
r_{k}=\frac{\sum_{i=1}^{N-k}\left(Y_{i}-\bar{Y}\right)\left(Y_{i+k}-\bar{Y}\right)}{\sum_{i=1}^{N}\left(Y_{i}-\bar{Y}\right)^{2}}
$$

The vector $Y_{i}$ is the input EEG data coming from the electrode analyzed, $i$ is the time index that represents the record sample, and $\mathrm{N}$ is the total number of samples in the data. Different values of $\mathrm{k}$ were used in the equation in order to select the optimum, but not any of them lead to any success in discriminating between the two groups of electrodes. In this study $\mathrm{k}$ was set to 10 . Observe in Figure 4.1 how these electrodes overlap with each other. 


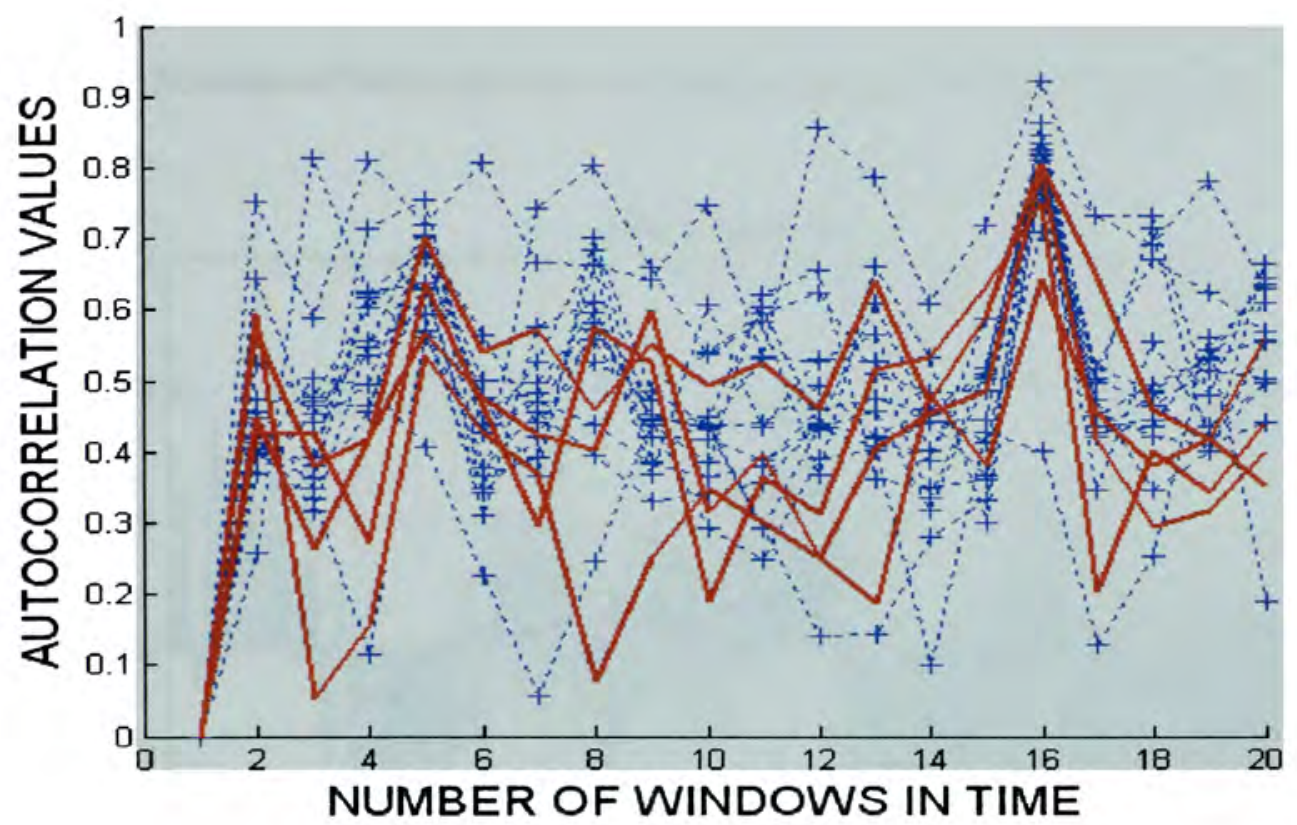

Figure 4.1. Autocorrelation values over time. Note: Leading to seizure (Red -) \& not leading (blue +-).

\subsubsection{AREA UNDER THE CURVE}

The area under the curve was also calculated in order to asses the fluctuations of the EEG channels using Equation 4.2. In this equation, $\mathrm{X}_{\mathrm{i}}$ is the vector that contains the samples of the electrode and $\mu_{\mathrm{i}}$ is the average of the sample values for a given electrode.

$$
\mathrm{A}=\sum_{\mathrm{i}=1}^{\mathrm{N}}\left|\mathrm{X}_{\mathrm{i}}-\mu_{\mathrm{i}}\right|
$$

In reference to Figure 4.2 , it is observed that the area under the curve of the EEG signal fluctuates with respect to the mean of the signal itself. As can be observed, the light blue color (shaded region) is the area calculated using Equation 4.2. A comparison 
of all the areas under the curve will be made in order to look for differences in the signature of the areas of those channels that lead to seizure and those that do not.

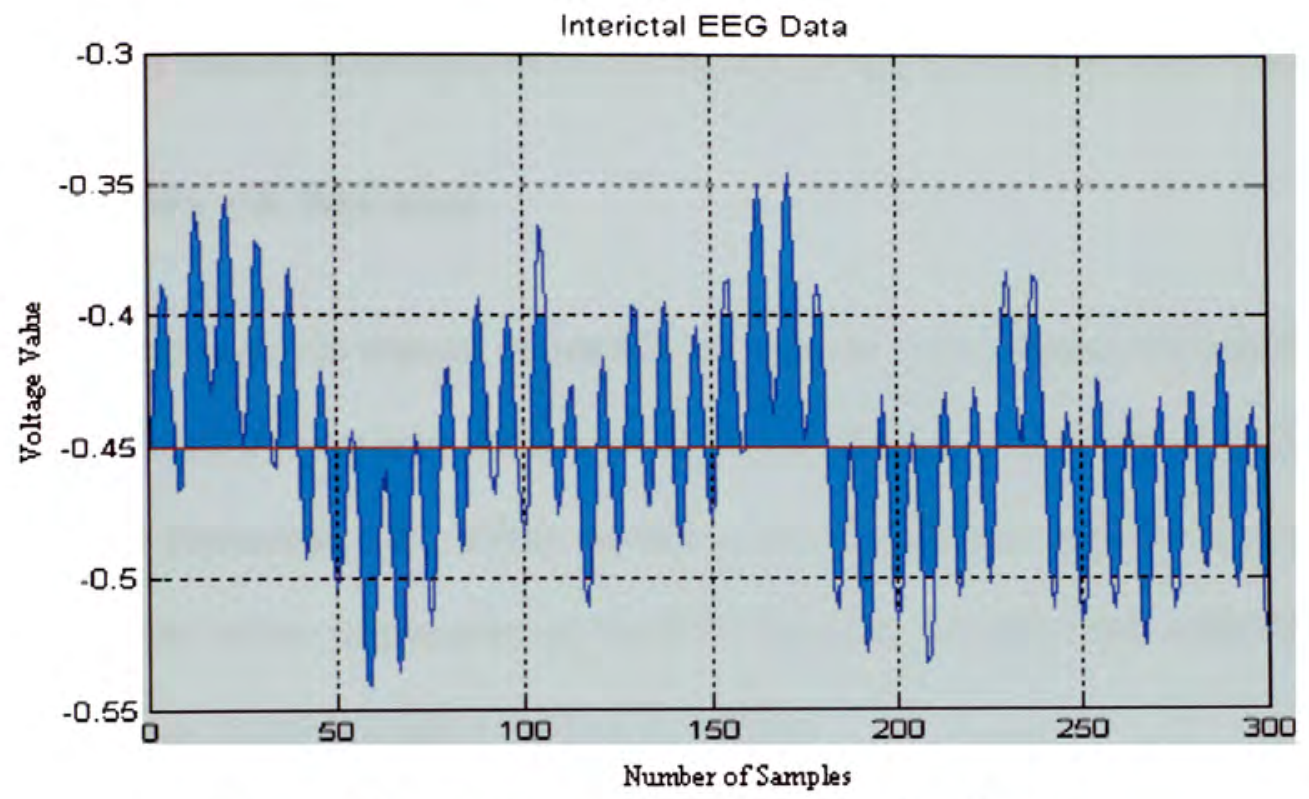

Figure 4.2. Representation of the area under the curve.

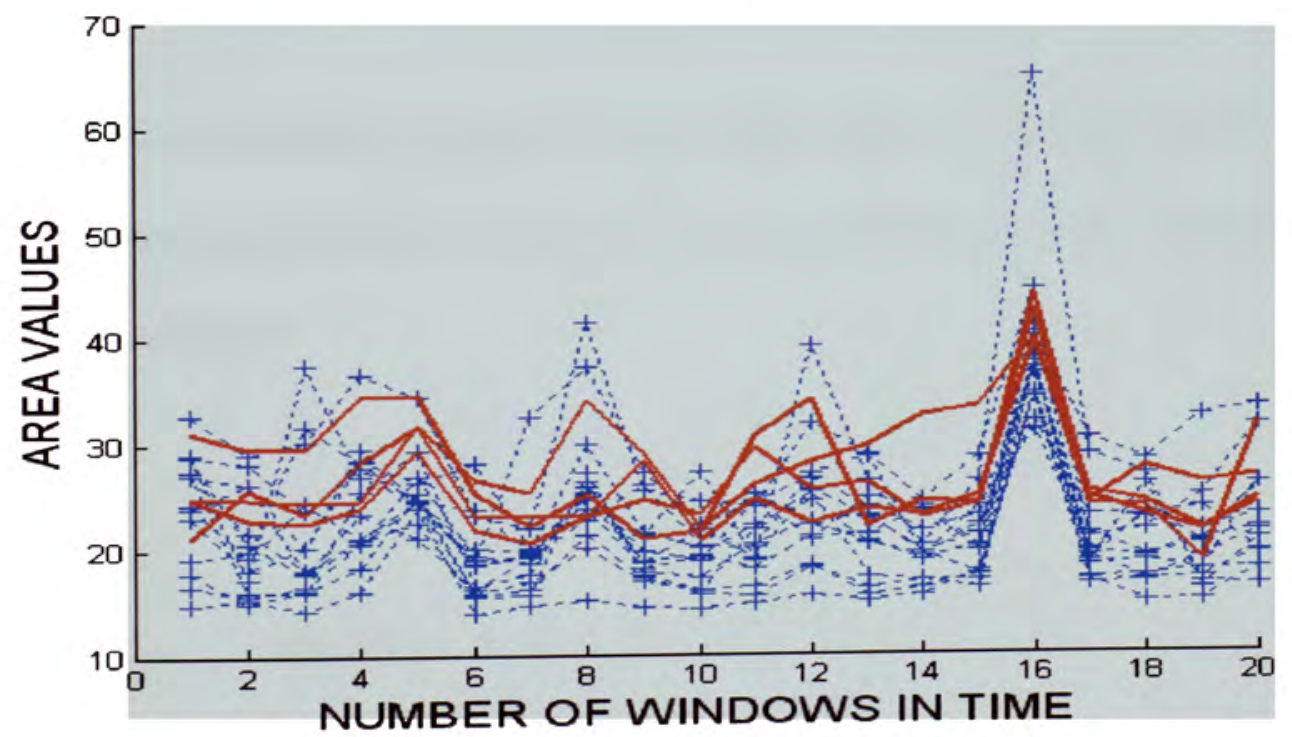

Figure 4.3. Representation of the area under the curve over time using equation 4.2. Note: Leading to seizure (Red -) \& not leading (blue +-). 
Nevertheless, once again this measure failed to discriminate between the two groups of electrodes as illustrated in Figure 4.3. Even though, the red and blue electrodes are kind of compact together, the red electrodes are mixed with them, and as a consequence, a reliable delineation between the two groups could not be performed.

\subsubsection{ENTROPY OF THE EEG}

The entropy is the amount of disorder of a system. It is a measure of information. The more information you have about a given system, the less uncertainty of that system is established (Shannon, C.E., 1948). In this study, the Shannon entropy was used in order to extract hidden information of the EEG signal of the electrodes analyzed. This Shannon entropy is defined and calculated as follows:

$$
H(x)=-\sum_{x=1}^{M} \sum_{i=0}^{L} P_{i} \log _{2} P_{i}
$$

Given an electrode $X$, where $X$ takes discrete values $x_{i}$ with probability $P_{i} . P_{i}$ is the probability that the signal belongs to a considered amplitude with $\mathrm{L}$ partitions, with the understanding that:

(a) $\sum_{i=1}^{\mathrm{L}} \mathrm{P}_{\mathrm{i}}=1$ with $\quad 0 \leq \mathrm{P}_{\mathrm{i}} \leq 1, \quad \mathrm{i}=1, \ldots \mathrm{L} \quad$ and

(b) $P_{i} \ln P_{i}=0$ if $P_{i}=0$ 
Where $\mathrm{M}=\mathrm{X}-\mathrm{W}$, and $\mathrm{W}$ is the window size chosen, in this case we used 2000 samples; also 10 partitions $(L=10)$ were used to calculate the entropy of the EEG signal. The representation of the values calculated for the entropy is displayed in Figure 4.4.

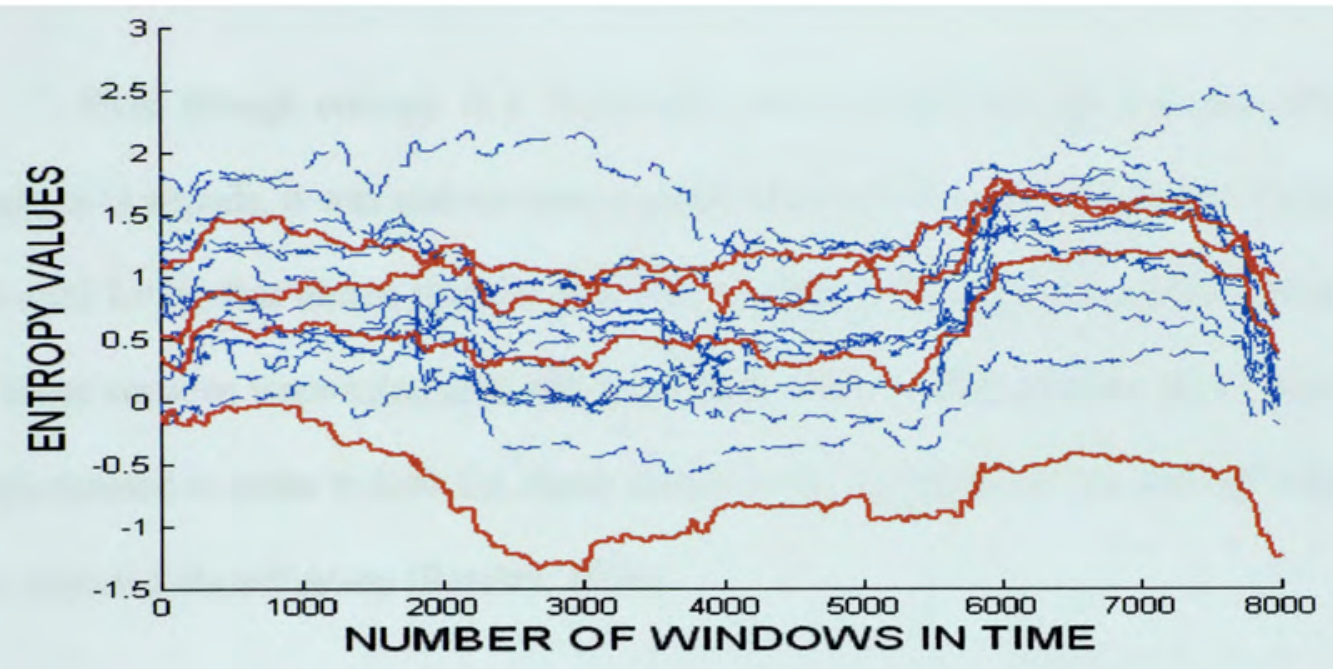

Figure 4.4. Entropy values over time. Note: Leading to seizure (Red -) \& not leading (blue --).

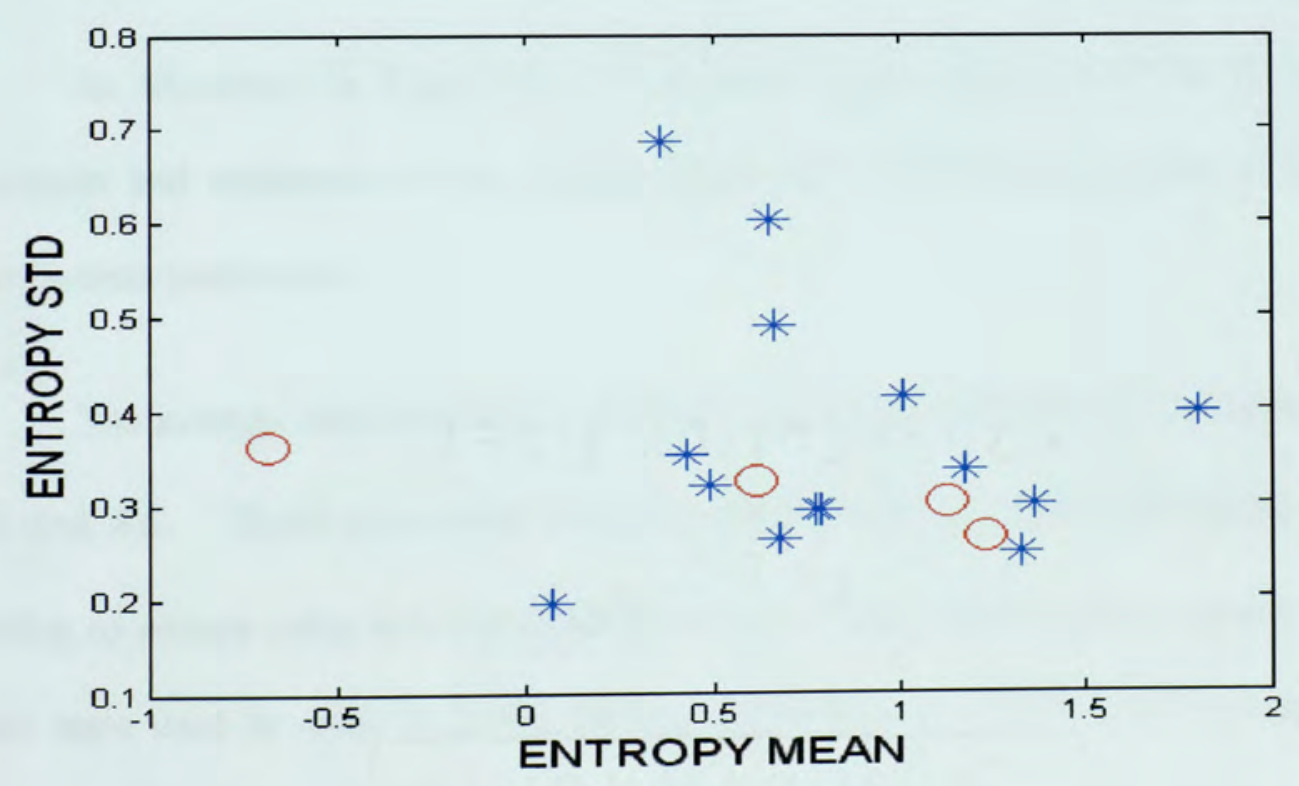

Figure 4.5 Entropy mean and STD values. Note: Leading to seizure (Red o) \& not leading (blue ${ }^{*}$ ). 
The entropy mean, that reflects mainly the changes in EEG spontaneous activity, and the standard deviation, that reflects the spike activity were also calculated. As illustrated in Figure 4.5, the Shannon entropy $(\mu \pm \sigma)$ is plotted using red and blue colors in order to analyze the fluctuations of these values for the two groups of channels.

Even though entropy is a commonly used equation for the detection of drastic changes in signals, it was also an unsuccessful parameter to use for our study. In this case we used $\mathrm{L}=10$, that means that the data was divided in 10 partitions and the probabilities of those sections were calculated and quantified. Also, another number of partitions were implemented in order to look for closer details in the signal, but it was still not helpful for the intended classification (Proakis, 1996).

\subsubsection{MINIMUM, MAXIMUM AND AVERAGE VALUES OF THE EEG SIGNALS}

As illustrated in Figure 4.6, the simplest measurements such as the average, minimum and maximum of the voltage amplitude of different segments of the EEG signals were performed.

The average, minimum and maximum values were calculated using equations 4.4 , 4.5, and 4.6. These parameters were performed for every electrode leading and not leading to seizure using non-overlapping windows of one second each. Several window sizes were used in order to attain for any differences, but there were not significant differences in the output results. 




(a) Minimum Values

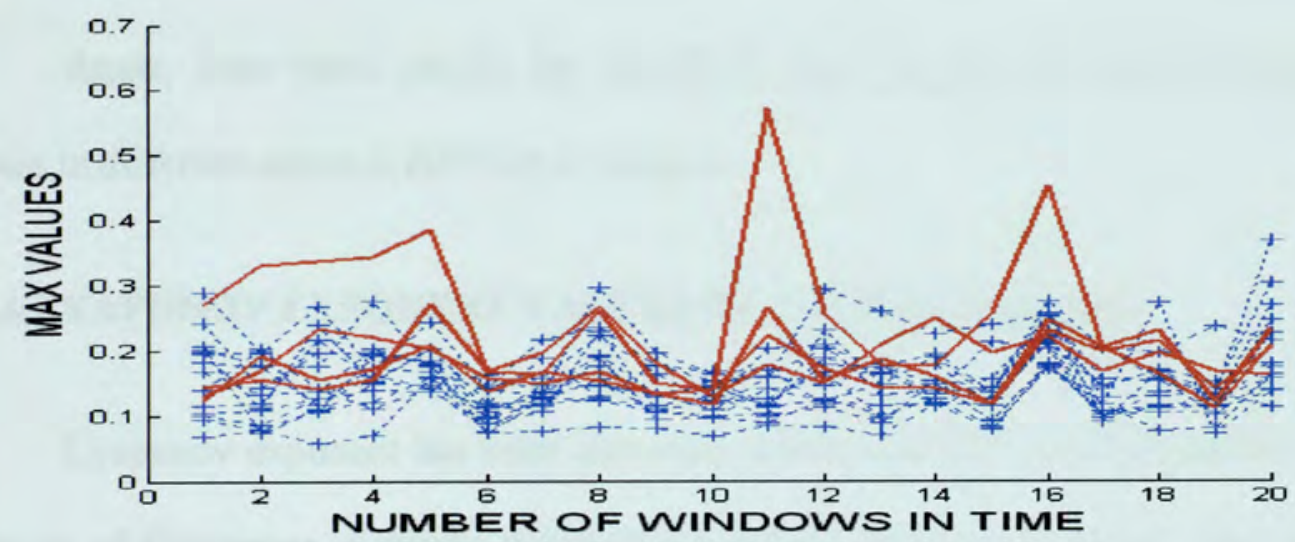

(b) Maximum Values

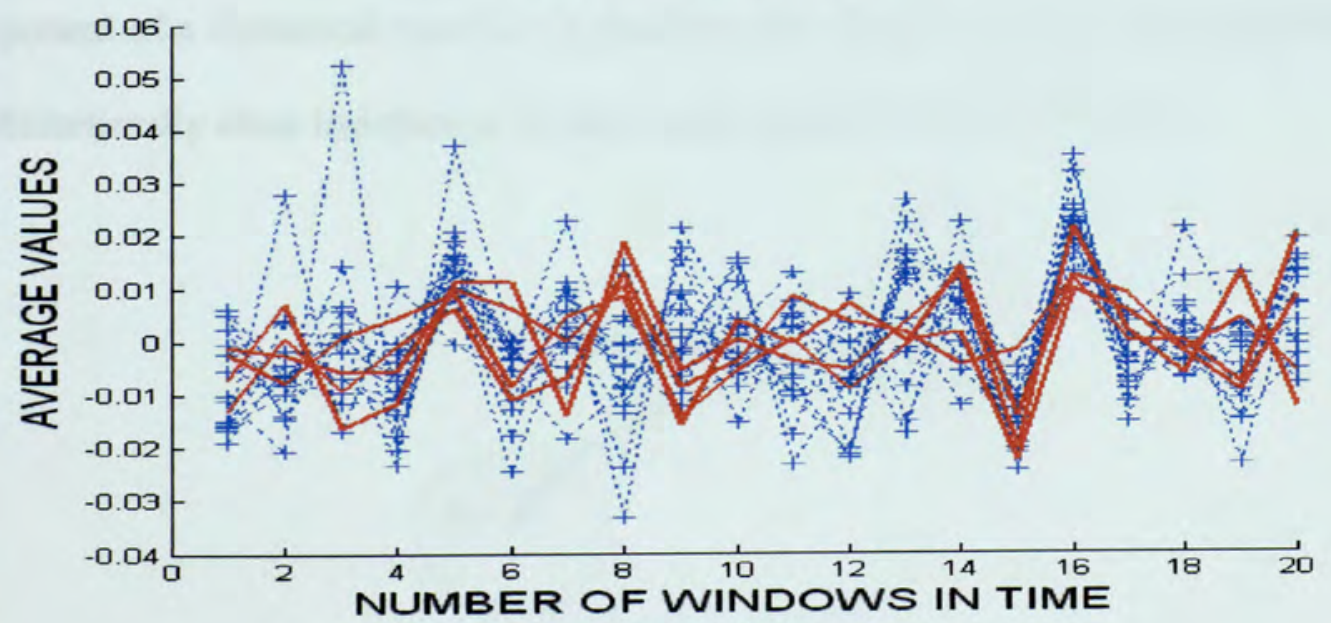

(c) Mean Values

Figure 4.6. EEG representation: (a) Minimum (b) Maximum (c) Mean. Note: Leading to seizure (Red -) \& not leading (blue +-). 
Where $\mathrm{N}$ is the total number of samples, and $\mathrm{X}_{\mathrm{i}}$ is the input signal, which in this case corresponds to every single electrode.

$$
\begin{aligned}
& \mu=\frac{1}{N} \sum_{i=1}^{N} X_{i} \\
& \operatorname{Min}=\operatorname{Min}\left\{X_{i}\right\} \\
& \operatorname{Max}=\operatorname{Max}\left\{X_{i}\right\}
\end{aligned}
$$

Again, from these results the delineation between the two types of electrodes shown in different colors is difficult to ascertain.

\subsubsection{LYAPUNOV EXPONENT VALUES OF THE EEG SIGNALS}

Lyapunov exponent has been also very widely used for seizure prediction. It is a measure of divergence of nearby trajectories and how chaotic is behaving. The Lyapunov exponent of a dynamical system is a quantity that characterizes the rate of separation of infinitesimally close trajectories in phase space as illustrated in Figure 4.7.



Figure 4.7. Flow of the Lyapunov exponent. 
The Lyapunov spectrum is also related with the entropy of the signal and can be used to estimate the rate of entropy production of the dynamical EEG system (Iasemidis, 1994). The Lyapunov exponent was calculated as follows:

$$
\mathrm{L}_{\mathrm{y}}=\frac{\sum_{\mathrm{m}=2}^{\mathrm{w}-\delta} \log _{2}\left(\frac{\mathrm{D}}{\mathrm{D}_{0}}\right)}{\mathrm{N}}
$$

Where $\delta$ is the dimension implemented in this study $(\delta=3)$ and $N$ is the total number of samples in the EEG data. The window size w was set to 500 samples. This value was chosen initially because the sampling rate of the input signal is 500 samples per seconds, but also other window sizes were implemented and the results did not change drastically. The term $\mathrm{D}_{0}$ that refers to the initial distance, and $\mathrm{D}$, which refers to the changing distances are calculated using Equations 4.8 and 4.9, respectively (Sprott, 2003; Hilborn, 2000).

$$
\begin{aligned}
& D_{0}=\sum_{m=2}^{w-\delta} \sqrt{\sum_{i=1}^{\delta}(\operatorname{Phase}(m-1, i)-\operatorname{Phase}(m, i))^{2}} \\
& D=\sum_{m=2}^{w-\delta} \sqrt{\sum_{i=1}^{\delta}(\operatorname{Phase}(m, i)-\operatorname{Phase}(m+1, i))^{2}}
\end{aligned}
$$

The embedding dimension implemented in this case was 3. Phase is the input vector that represents the EEG electrode. 
Even though the Lypaunov exponent has been successfully used for seizure prediction, a simple analysis performed using this parameter failed to discriminate the two groups of electrodes as illustrated in Figures 4.8 and 4.9 for the considered windows of time.

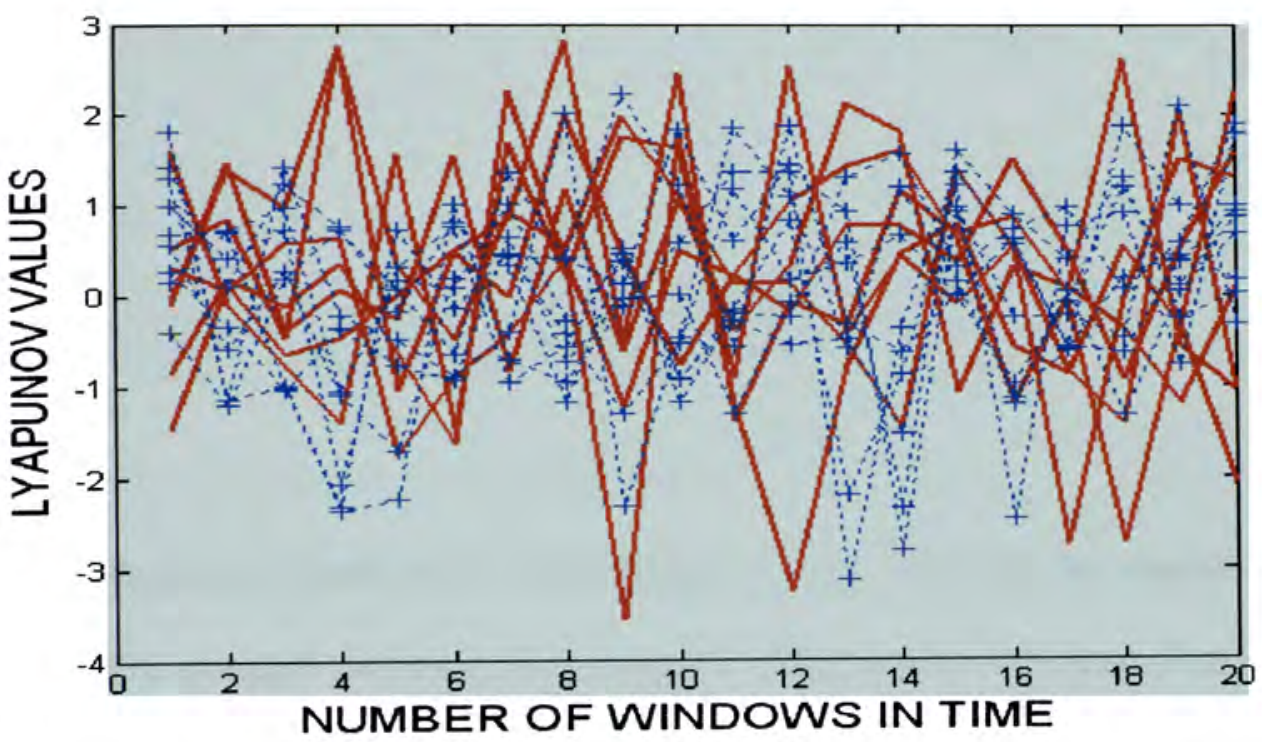

Figure 4.8. Lyapunov exponents over time. Note: Leading to seizure (Red -) \& not leading (blue +-).

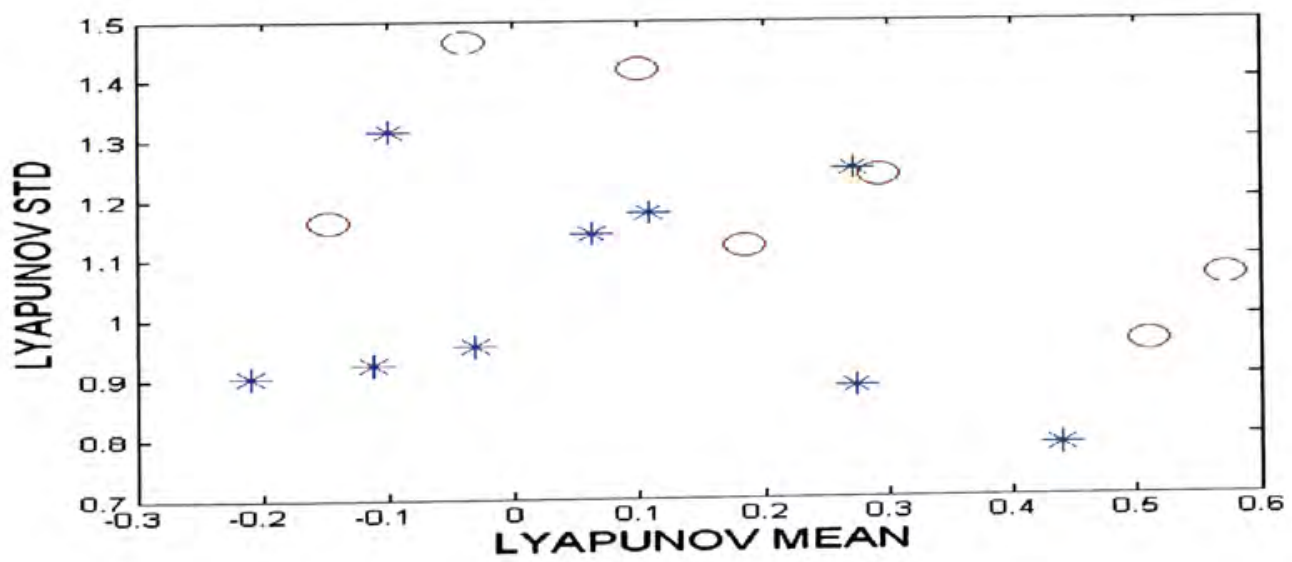

Figure 4.9. Mean and STD values of the Lyapunov exponent. Note: Leading to seizure (Red o) \& not leading (blue *). 


\subsubsection{ACTIVITY OF THE EEG SIGNALS}

The activity or the variance of the EEG signal is also investigated in this research. The variance of the amplitude fluctuations in the EEG epochs is analyzed. Equation 8 was used to calculate the values where $X$ is the input EEG electrode, $\mu$ is the average value of the voltage for a given electrode, and $\mathrm{N}$ is the total number of samples in the data.

$$
A_{c}=\sigma^{2}=\frac{\sum_{i=1}^{i=N}(X(i)-\mu)^{2}}{N}
$$

As shown in Figure 4.10, this measure is not sufficient for classification of electrodes. The standard deviation with respect to the mean of the signal does not show significant patterns for delineation of the two groups of electrodes.

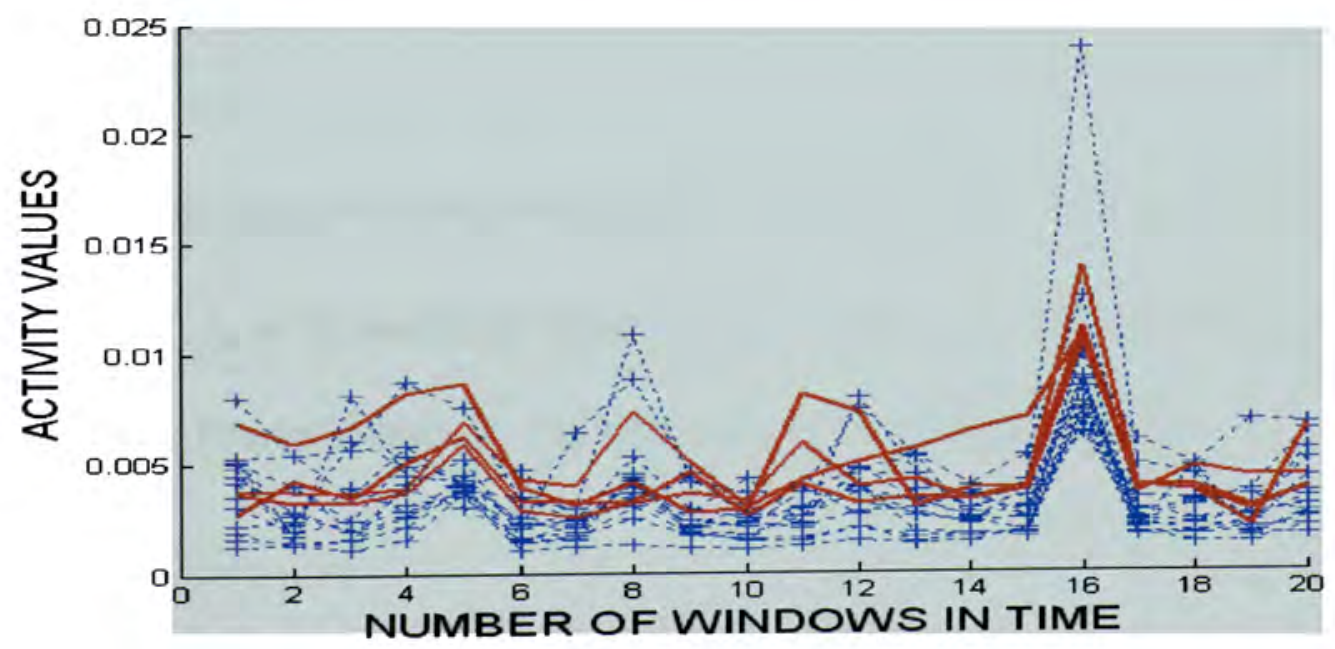

Figure 4.10. Activity values over time. Note: Leading to seizure (Red -) \& not leading to seizure (blue +-). 


\subsubsection{COHERENCE OF EEG SIGNALS}

The coherence was used in order to perform the analysis and draw insight from the spectra of the EEG signals. Representations based on Fourier Transforms are useful in this case. This approach considers the EEG spectrum that falls primarily within fourfrequency bands-alpha $(8-13 \mathrm{~Hz})$, beta $1(13-20 \mathrm{~Hz})$, beta $2(20-30 \mathrm{~Hz})$, delta $(1-4)$, and theta $(4-8 \mathrm{~Hz})$.

The Fast Fourier Transform for each electrode was calculated and the mean voltage and the relative mean power of every frequency band was considered.

The mean voltage amplitude $(\bar{V})$ and the relative mean power $\left(\overline{\mathrm{P}_{\mathrm{r}}}\right)$ for each band were calculated using Equations 4.11 and 4.12.

$$
\overline{\mathrm{V}}=\frac{1}{\mathrm{~N}} \sum_{\mathbf{w}=\mathrm{i}}^{\mathrm{j}} \mathrm{X}^{\prime}(\mathrm{w})
$$

Where, $i=f_{C 1} \frac{N}{2} \cdot 2 \cdot f_{s} \quad$ and $\quad j=f_{C 2} \frac{N}{2} \cdot 2 \cdot f_{s}$

In this case $N$ is the total number of samples, $\mathrm{f}_{\mathrm{Cl}}$ and $\mathrm{f}_{\mathrm{C} 2}$ are the cut off frequencies, $f_{s}$ is the sampling frequency and $\mathrm{X}^{\prime}(\mathrm{w})$ is the vector containing the real values of the Fourier Transform. The relative mean power is calculated by

$$
\overline{P_{r}}=\frac{\frac{1}{N} \sum f b^{2}(i)}{\frac{1}{N} \sum a f b^{2}(i)}
$$


Where $\overline{P_{r}}$ denotes for relative mean, $\mathrm{fb}$ represents the voltage values for a specific band, the afb values represent the voltage values for all the bands, and $N$ is the total number of samples in the input EEG signal.

The following formula was employed to calculate the coherence values:

$$
K(f)=\frac{\left|\sum_{i=1}^{N} P_{x y}(f, i)\right|^{2}}{\sum_{i=1}^{N} P_{x x}(f, i) * \sum_{i=1}^{N} P_{y y}(f, i)}
$$

$$
P_{x x}(f, i)=X_{i}(f) * X_{i}^{*}(f)
$$

and,

$$
\begin{aligned}
& P_{y y}(f, i)=Y_{i}(f) * Y_{i}^{*}(f) \\
& P_{x y}(f, i)=X_{i}(f) * Y_{i}^{*}(f)
\end{aligned}
$$

$P_{x x}$ and $P_{y y}$ are the power spectrum corresponding to the pair of electrodes analyzed, and $P_{x y}$ is the cross spectrum between the two electrodes. $X_{i}(f)$ and $Y_{i}(f)$ are the vectors containing the frequency components of the EEG input vectors. $X_{i}^{*}(f)$ and $Y_{i}^{*}(f)$ are the complex conjugate vectors of $X_{i}(f)$ and $Y_{i}(f)$, respectively.

The EEG coherence analysis is designed to find out whether the brain waves from two different parts of the brain are synchronized. The EEG coherence was calculated for the alpha activity of different combinations of pair of electrodes. Each pair consists of one electrode that lead to seizure and the other will be its nearest electrode that does not 
lead to seizure. The results demonstrated that for those electrodes that lead to seizure, the coherence values were higher than for the electrodes that do not lead to seizure.

\subsection{EXTRACTION OF BEST PARAMETERS}

At this stage of the investigation, a few parameters have been tried and tested in the EEG data, such as voltage (maximum, minimum, average and standard deviation), power spectrum in the frequency bands alpha, beta, delta and theta, autocorrelation, the Hjorths' parameter activity, the autocorrelation, the Lyapunov exponent, voltage area under the curve, and coherence. Nevertheless, a detailed analysis based on similarity searching yielded that the parameters that best discriminate in advance the two classes of electrodes were the complexity, correlation integral, and mobility, which will be explained with more detail in this section.

The main idea of this study is to investigate if the use of the following parameters could adequately answer the question of any similar behavior of the channels that lead to seizure, in cases where it is already known that the spikes are independent. In the evaluation of all relevant parameters that could potentially asses the dynamics of EEG, it was revealed that correlation dimension $(\mathrm{R}(\mathrm{r}))$, Mobility $(\mathrm{M}(\mathrm{y}))$ and Complexity $(\mathrm{C}(\mathrm{y}))$ performed the best for the research goals of this dissertation. These key parameters were computed using non overlapping sliding windows of 1 second for all electrodes (Yaylali, 1996).

Since brain dynamics are nonlinear, this study investigated the calculation of the correlation dimension integral (Proakis, 1996; Iasemidis, 1994). The correlation 
dimension integral $\mathrm{R}(\mathrm{r})$ given in equation 4.15 is a measure of spatial organization, where the space is occupied by a set of random points.

$$
R(r)=\frac{1}{N^{2}} \sum_{j=1}^{N-1} \sum_{i=j+1}^{N-1} \theta\left(r-\left|X_{i}-X_{j}\right|\right)
$$

Where, $r$ is the threshold value used to evaluate the similarity between two reconstructed vectors $X_{i}$ and $X_{j} . N$ is the total number of points in the time series. The vector $X_{i}$ is a point in the embedded phase constructed from the input EEG signal as a single time series according to the following formula:

$$
X_{i}=\left(X_{i}, X_{i}+\tau, X_{i}+2 \tau, \ldots X_{i}+(m-1) \tau\right) \text {, where } m \text { is the so called embedding }
$$

dimension and $\tau$ is a time delay. In Equation 4.15 the counting of the distances between the chosen points in the phase space is symbolized by the so called Heaviside unit step function $\theta(x)$, where $\theta(x)=0$ for $x \leq 0$, and $\theta(x)=1$ for $x>0$. The correlation integral averages the probability of measuring a distance smaller than $r$ between two randomly chosen points $X_{i}$ and $X_{j}$.

Additionally, the Hjorth's parameters, mobility and complexity were calculated using Equations 4.16 and 4.17, respectively. Mobility gives a measure of deviation of the voltage changes with respect to deviation of the EEG voltage amplitude, while complexity (Equation 4.17) provides a measure of excessive details with regard to the 
slightest possible signal's shape (Gevins, 1987). The mobility is computed using the following formula.

$$
\mathrm{M}(\mathrm{y})=\sqrt{\frac{\sigma\left(\mathrm{y}^{\prime}\right)}{\sigma(\mathrm{y})}}
$$

Where $\sigma$ is the variance and $y^{\prime}$ is the first derivative of the primary input EEG signal $\mathrm{y}$. The complexity $\mathrm{C}(\mathrm{y})$ is the square root of the ratio between the mobility of the first derivative of the signal $M\left(y^{\prime}\right)$ and the mobility of the signal itself $M(y)$ and it is expressed as:

$$
C(y)=\sqrt{\frac{M\left(y^{\prime}\right)}{M(y)}}
$$

\subsection{PRELIMINARY ANALYSIS TO THE EEG SIGNAL}

It is important to emphasize at this time that once the best parameters were chosen to process our data, key conditions were drawn out prior to processing EEG data in order to accomplish the electrode delineation/classification problem. First, it was determined that electrodes located in the same region tend to behave very similar to one another. Second, it is significant to make sure that the interictal spikes are firing at different times, and in concert to the first condition, that these electrodes have to be situated in different regions in the brain. 


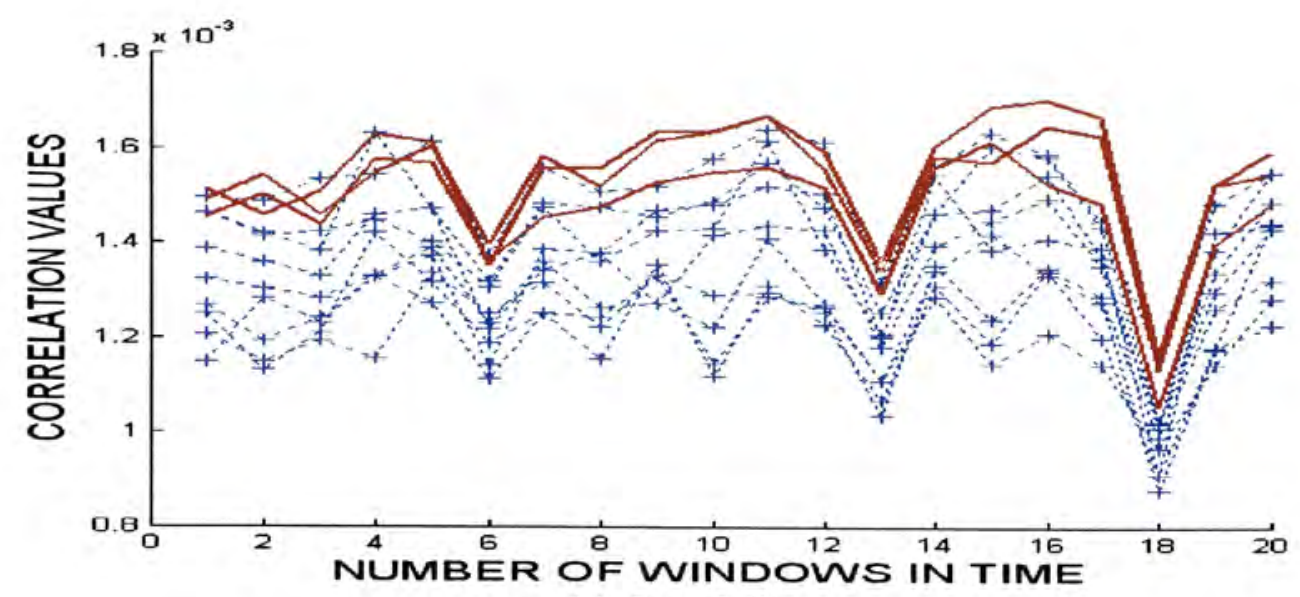

(a) Correlation



(b) Mobility

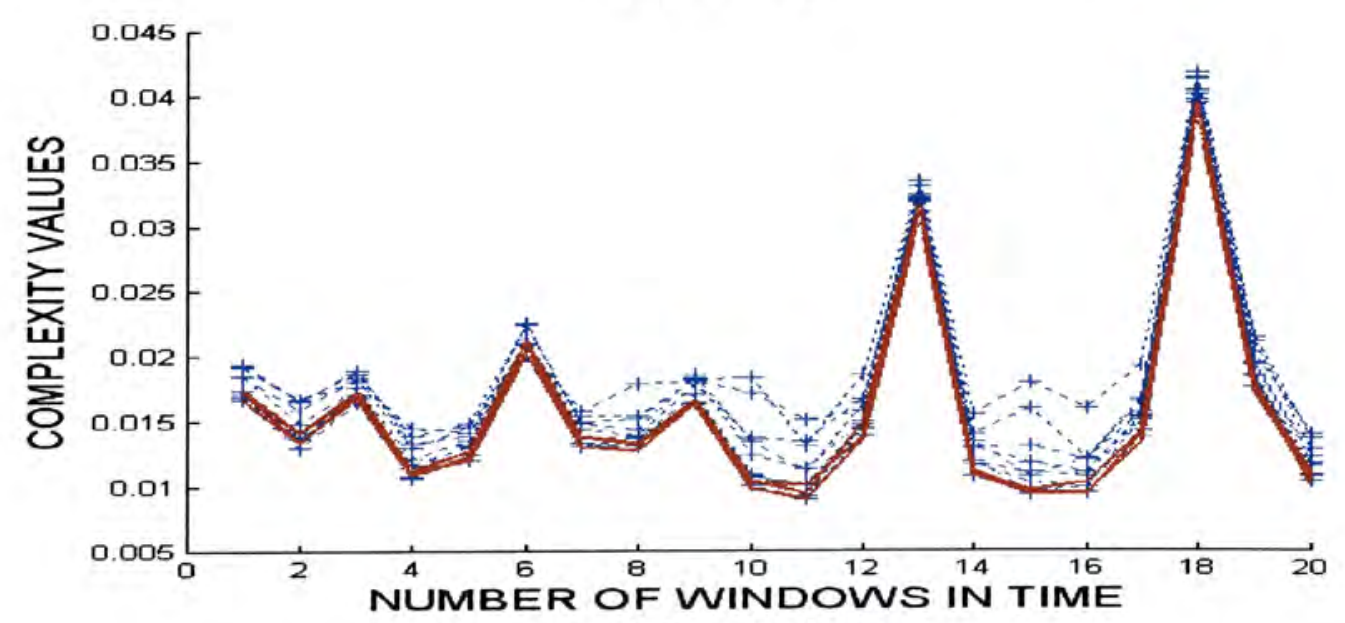

(c) Complexity

Figure 4.11 Correlation, mobility, and complexity with electrodes belonging to a single region (patient 12). Note: Leading to seizure (Red -) \& not leading to seizure (blue +-). 
For illustrative purposes, the correlation dimension, mobility, and complexity parameters are displayed over time in Figure 4.11. These parameters could be used in discriminating between electrodes that lead or do not lead to seizure, but in this case since the region of the electrodes analyzed is the same and the spikes are synchronously firing, there was a lot of overlap, thus a clear separation of them is unyielding.

\subsubsection{WALSH TRANSFORM APPLIED TO THE EEG AS A PRE-FILTERING}

\section{STEP}

The Walsh operators, as explained earlier in chapter 3 , are related to the mathematical derivatives. These functional equivalencies are mentioned here to show that Walsh operators just like derivatives can indeed be used to extract sharp transitions and pinpoint through zero-crossings the location of a given change in the EEG signal.

The first and second operators of the Walsh Transform were applied to the EEG signal as a preprocessing step to account only for the relevant changes in EEG. As exemplified in Figure 4.12, the correlation results discarding the noise effect before and after implementing the Walsh operators are not that different from each other. The results after applying the Walsh Transform look more spread in the 2D plot, but still there is one overlap. Also, the separation between the two classes of electrodes is more distinct after applying the Walsh Transform (Gonzalez, et. al., 1993). 


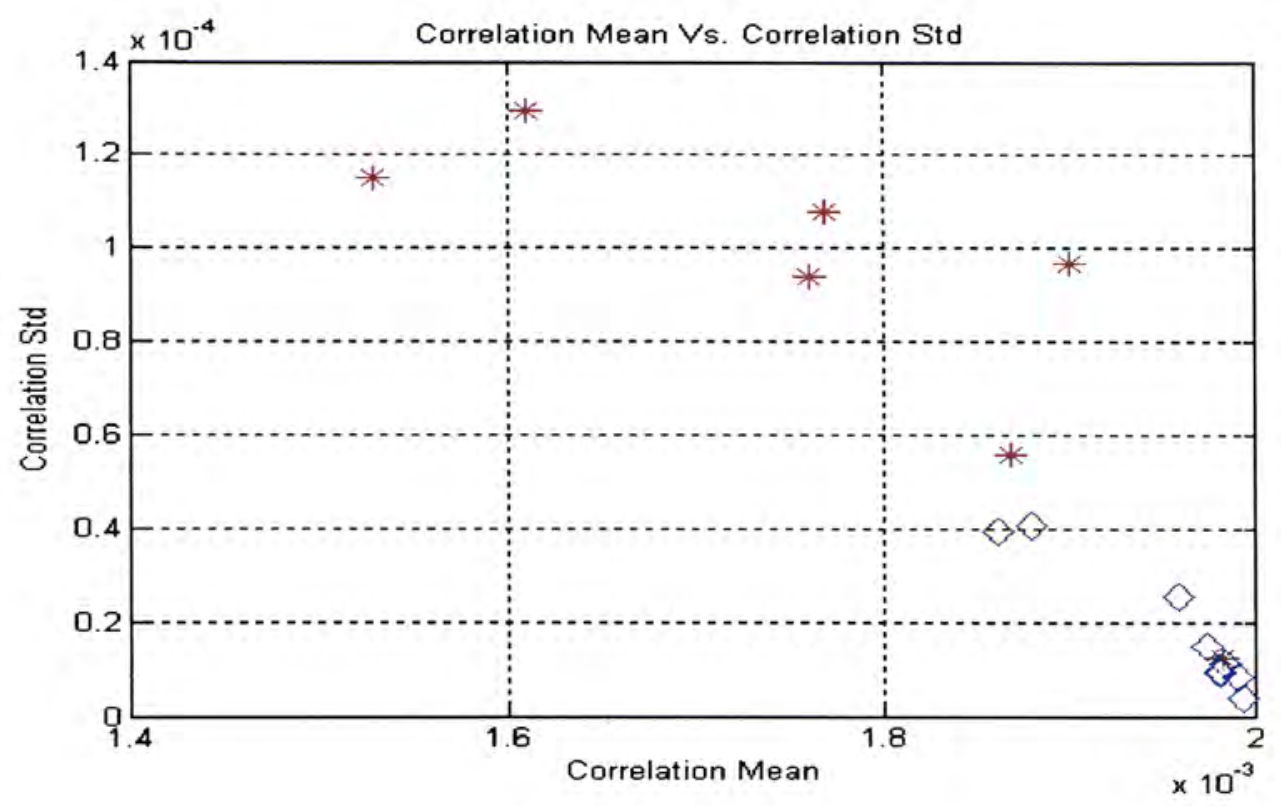

(a) Before Walsh Transform

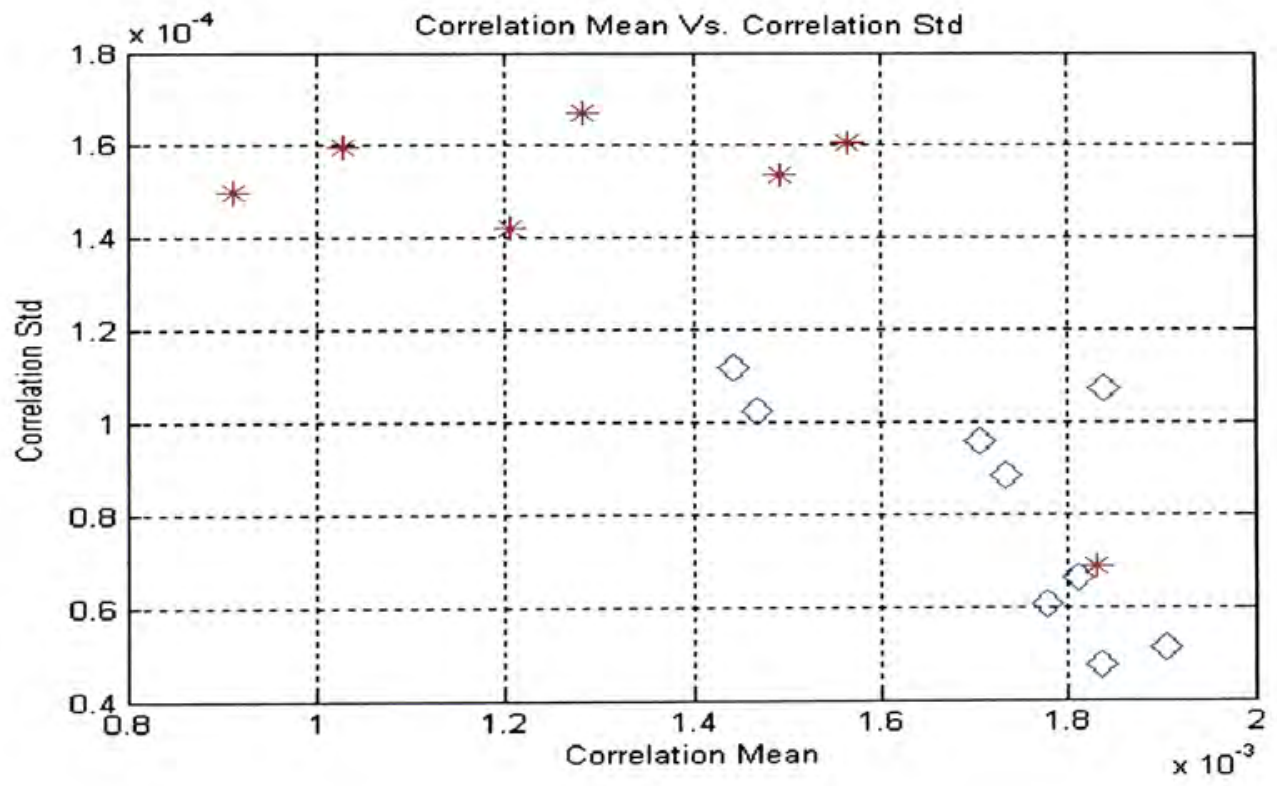

(b) After Walsh Transform

Figure 4.12. Before and after Walsh Transform. Note: Red stars (leading to seizure) \& blue diamonds (not leading to seizure). 
Even though, the Walsh Transform was implemented and tested in our data, it was not used as a pre-filtering step for the 8 patients analyzed in this study, since the results did not show a significant change for those patients, where overlapping is not an issue. This step was skipped in order to reduce the processing time of the algorithm. Nevertheless, this approach was used for the rest of the patients, where there was significant overlap. In this way, the results were better separated from each other.

\subsection{INTERPRETATION OF THE KEY PARAMETERS IMPLEMENTED IN THIS STUDY}

The motivation is to determine differences, if any, of those electrodes with interictal spikes activity (I.S.A.) that lead to seizure in contrast to those electrodes with I.S.A., but did not lead to seizure. It is hoped that such new methodologies will elicit new understanding towards the complex and still unresolved problem of seizure prediction.

This section provides technical details on the behavior of these parameters on well known functions. Different types of signals were used in this endeavor, such as the Sinc function, Sawtooth function, and the sine and cosine functions using different range of frequencies The motivation here is to understand how such parameters react to the dynamics of different input signals, in preparation to using EEG data, and with the purpose to draw a reliable conclusion from this knowledge.

The first function used as input data was the Sinc function. As it can be observed from Figure 4.13 , the mobility and complexity results are similar with different scale values, and the correlation is just the same, but inverted. 
The function implemented next is the so called Sawtooth function with a frequency of $50 \mathrm{~Hz}$ as illustrated in Figure 4.14. The results for the 3 parameters are straight lines because these functions are periodic; so it makes no difference to apply any of those parameters in this specific case. Although small differences can be noticed, given a different scale, the results of the mobility and complexity are straight line approximations.

Also, the inverse sawtooth as shown in Figure 4.15 is applied in order to see any differences with the sawtooth shown before and the results are the same, just a straight line. Therefore, the polarity of the input values it does not matter as long as the shape and periodicity of the signal is kept.

A sine function was implemented with a frequency of $100 \mathrm{~Hz}$ as illustrated in Figure 4.16. The results for the 3 parameters are also a straight line. It seems that if the signal is periodic, the output will be constant.

Another sine function was implemented with a frequency of $200 \mathrm{~Hz}$ as shown in Figure 4.17. In this case we doubled the frequency hoping to observe some changes, trying to notice if the frequency of the oscillation of the signal influences the output results. No change is noticed since the results for the 3 parameters are also constant.

In Figure 4.18, two combined sine functions were implemented with a frequency of 50 and $120 \mathrm{~Hz}$. The results for the 3 parameters are also a straight line. 

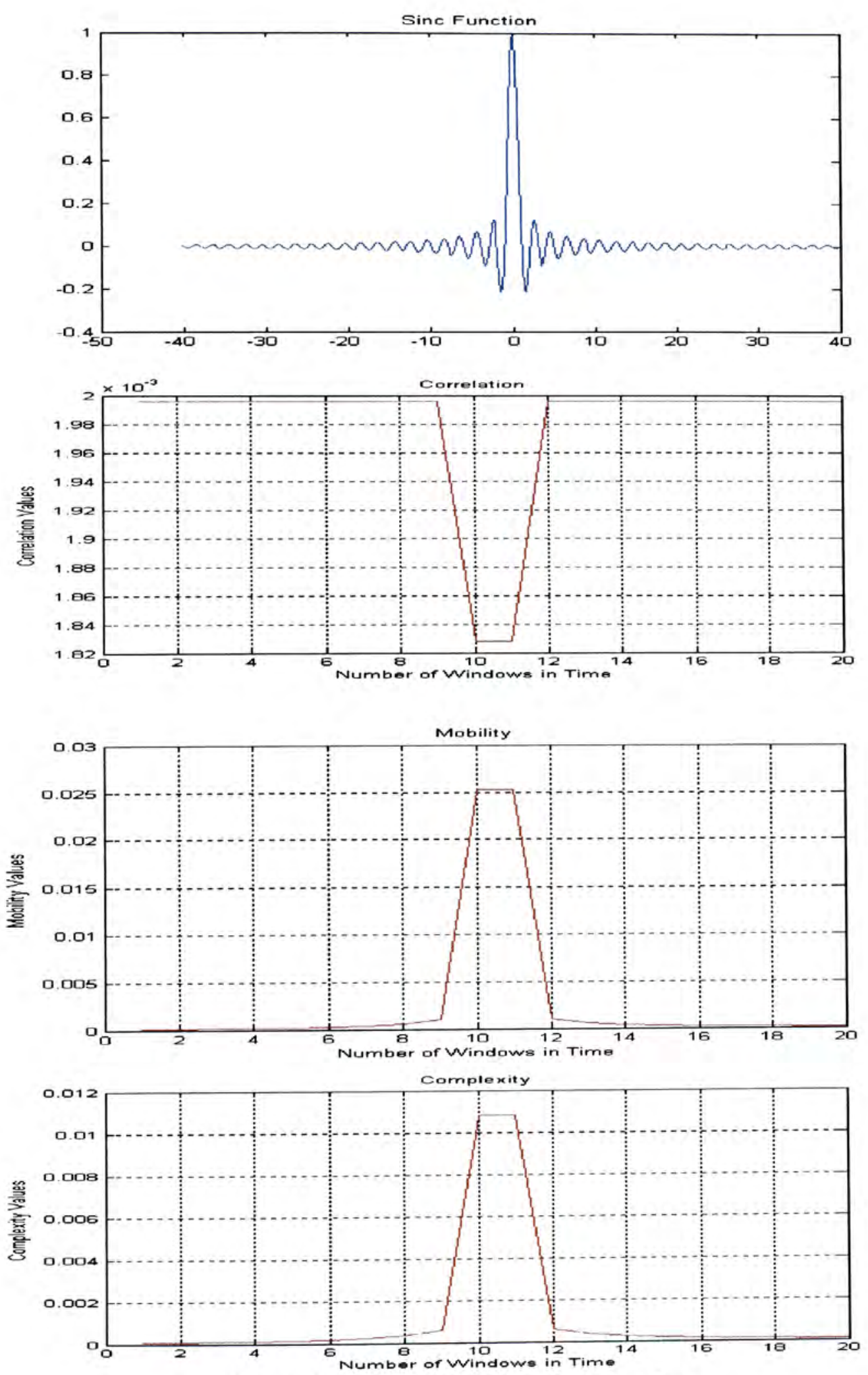

Figure 4.13. Analysis of the sinc function. 

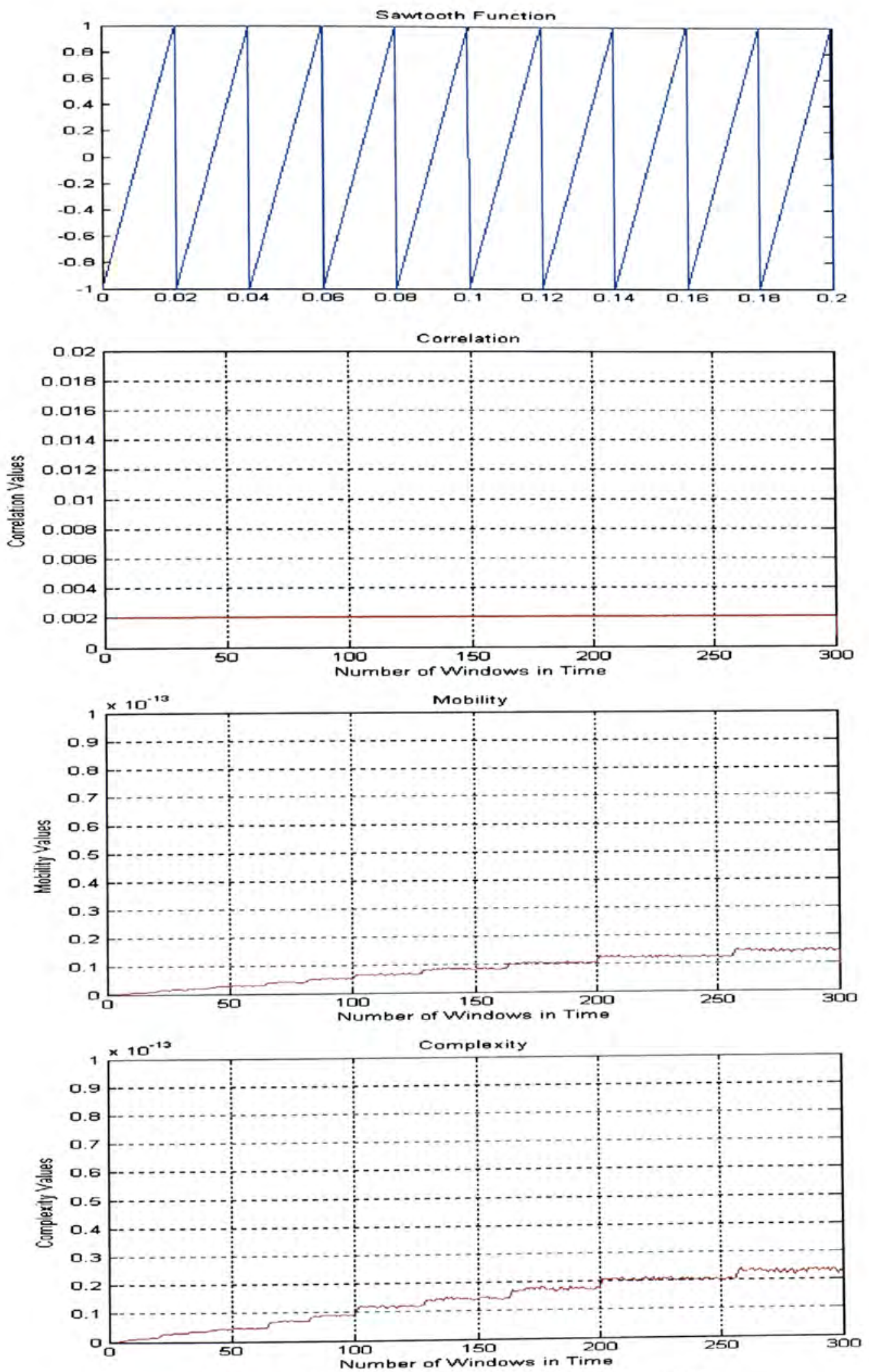

Figure 4.14. Analysis of the sawtooth function. 

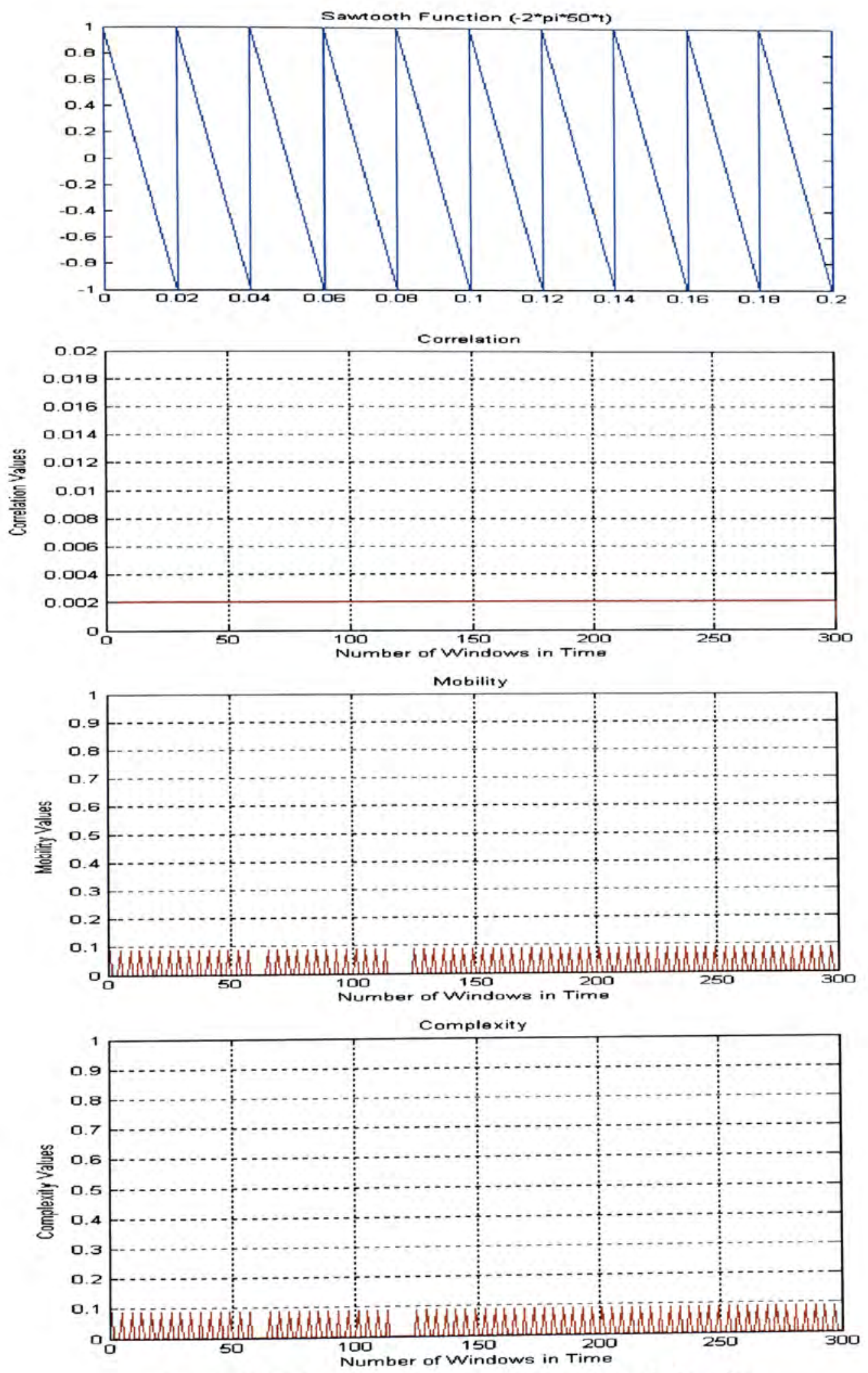

Figure 4.15. Analysis of the inverted sawtooth function. 

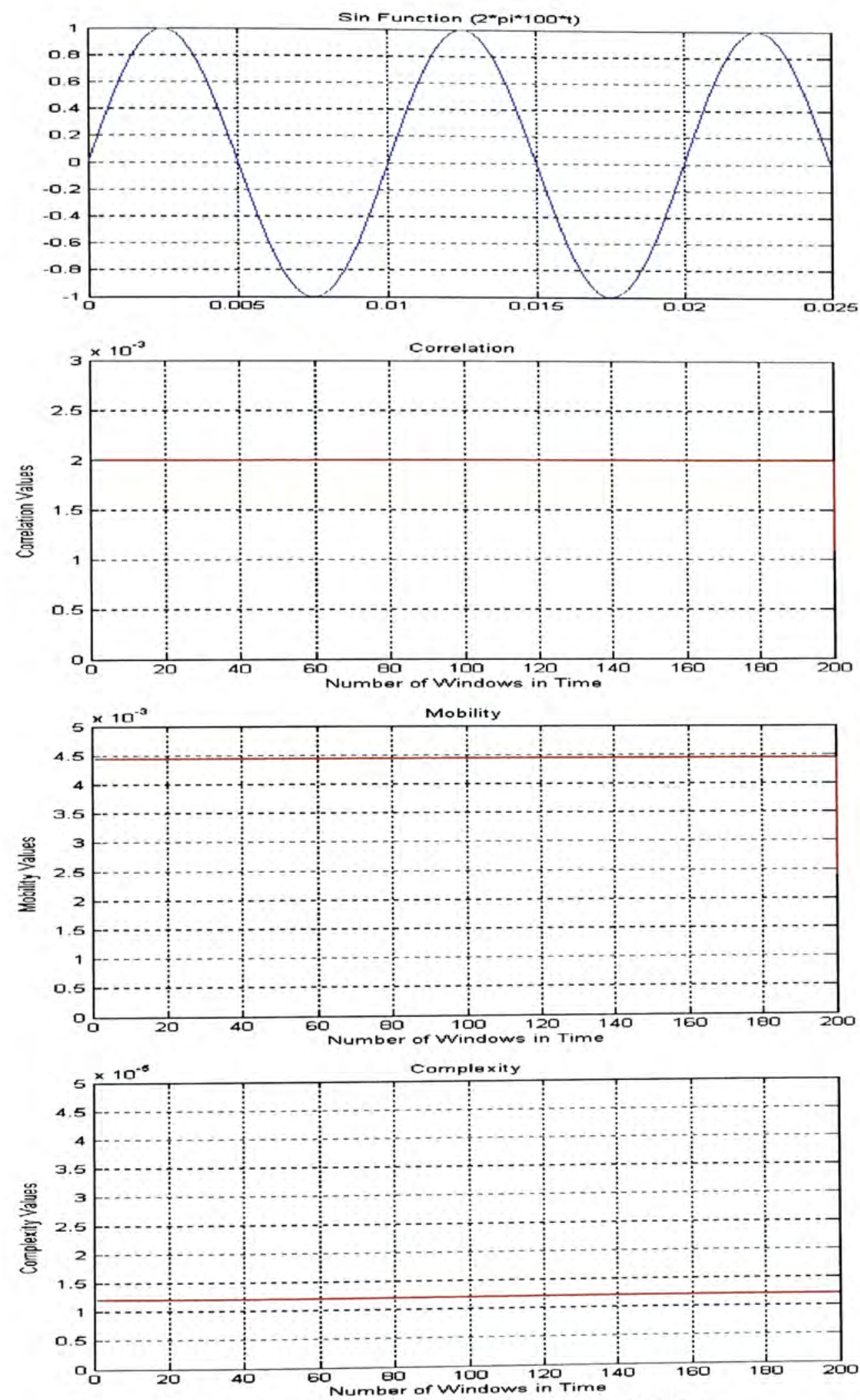

Figure 4.16. Sine function analysis $(f=100 \mathrm{~Hz})$. 



Figure 4.17. Sine function analyses $(f=200 \mathrm{~Hz})$. 

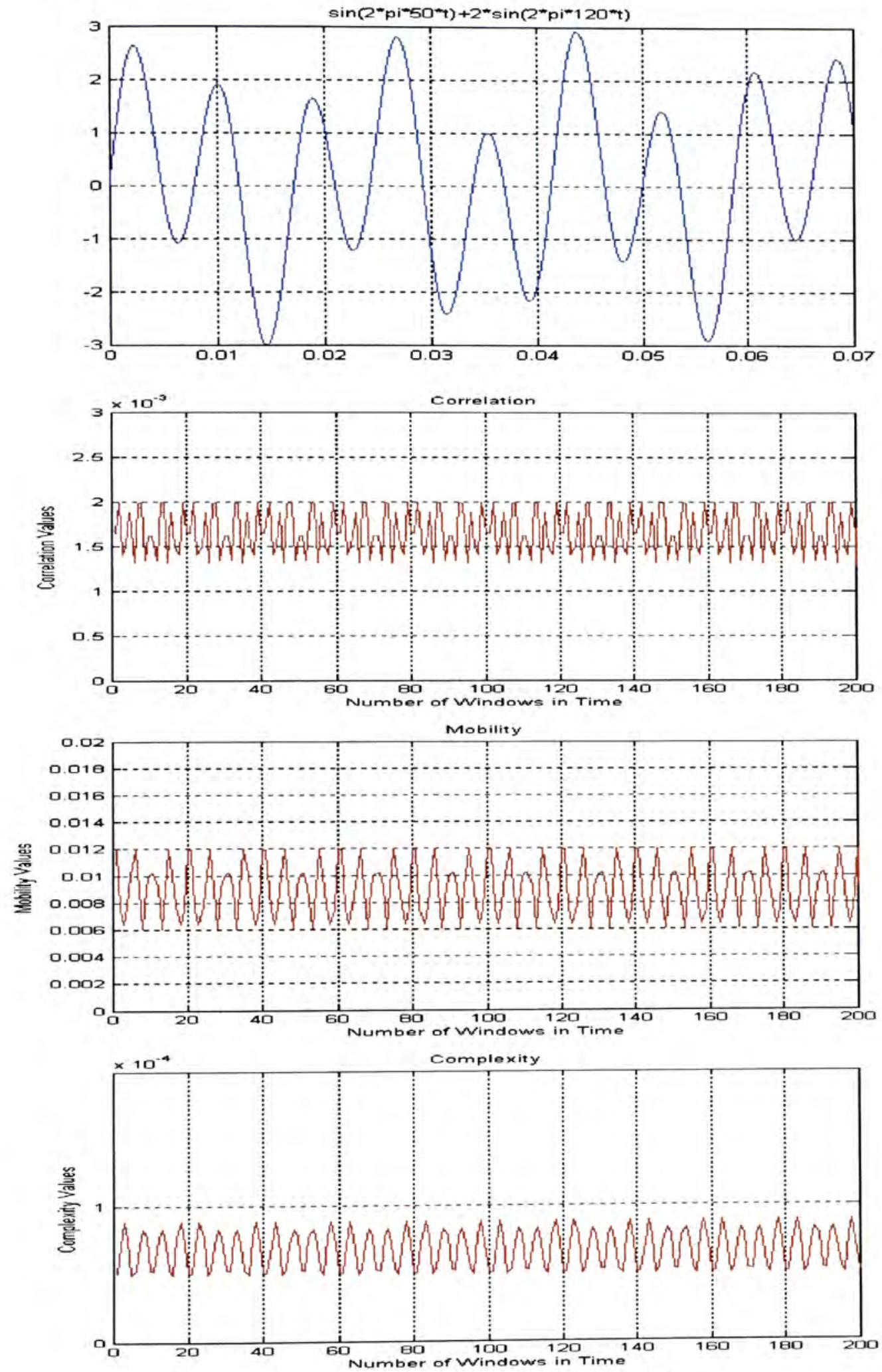

Figure 4.18. Two sine functions analyzed. 


\subsection{RETROSPECTIVE}

This initial study described in this section is to serve as a precursor to the next study, in view of our augmented understanding of such interictal subdural EEG recordings. Having established our framework for interictal EEG understanding using different parameters to analyze the data, we have expanded this work in view of our collaboration with our colleagues from Miami Children's Hospital to EEG activities focusing on epileptic seizures. This leads us to a novel approach that constitutes the main contribution of this dissertation. Such an approach shows great promise, as shall be described in the next chapter, in that it is integrated and operates effectively on interictal EEG signals for the delineation of channels that lead to seizure from those channels that do not. 


\section{CHAPTER 5}

\section{INTERICTAL EEG DATA PROCESSING AND RESULTS}

\subsection{INTRODUCTION}

Surgical interventions are now being increasingly performed in children with intractable seizures. These seizures often arise from extra-temporal sites and are related to a malformed pathologic substrate that is usually more extensive than the lesion evident on MRI scans (Ebersole, 2000). Presurgical evaluation relying on EEG data analysis including characterizing interictal spike discharges (Barreto, 1993) is consequently deemed critical towards taking measured steps for a successful outcome. EEG interictal data, as illustrated in Figure 5.1, recorded using subdural electrodes (on the cortical surface of the brain) can be processed to define similar/dissimilar patterns evident in those electrodes that lead to seizure to further facilitate surgical planning.

Operative success hence depends to a large degree on the results of a comprehensive pre-operative patient evaluation, the main purpose of which is to delineate the epileptogenic lesion and potentially its causation, especially when all test results point to a single epileptogenic focus.

The EEG of epileptic subjects is commonly divided into two main categories, the interictal and ictal EEG. The interictal EEG is the EEG data subset taken when the patient is not having seizures or in between seizures. In other words, interictal epileptiform activity is considered to be abnormal activity that can occur in a patient with epilepsy in 
the absence of an actual seizure. The ictal EEG activity on the other hand is when the actual seizure occurs (Greenstein, 2000; Martini, 2001).



Figure 5.1. Interictal EEG data (red electrodes (lead to seizure) \& blue electrodes (do not lead to seizure).

This dissertation introduces a mathematical framework for a new application dedicated to extract quantifiable measures that discriminate EEG patterns that lead to seizure from those that do not. This process is to be accomplished even when both classes of patterns contain interictal spikes that are used as indicators or precursors to the onset of such seizures. The final and most important goal will be in determining the key 
parameters and the related decision functions that permit for such delineation between the two types of electrodes. One nonlinear measure, the correlation integral, and two descriptive measures proposed by Hjorth (mobility and complexity) are used as the key parameters to assess the dynamics of the EEG data prior during and after an epileptic seizure.

A persisting dilemma that neuroscientists face in this research direction is in the inability to discern the reason as to why not all EEG channels that present interictal activity lead eventually to an ictal state (seizure). It is imperative then to establish the groundwork for experimental evaluations that will address such a dilemma. It is strongly believed that if a method is to be developed in order to delineate those electrodes that present interictal activity and do lead to an epileptic seizure, then certainly (a) the epilepsy focus could be located in a more optimal fashion, (b) further scrutiny of behavioral trends of these particular electrodes could potentially reveal new predictors of seizures, and (c) refined analysis of this electrode delineation process could also lead to a new outlook on the relative placement of the electrode arrays, which can only bring added credence to the point made in (a).

\subsection{METHOD}

This section provides an overview of the participants, recording equipment used, the data analysis required, and the general steps of the integrated algorithm proposed in this dissertation. 


\subsubsection{PARTICIPANTS}

Table 5.1. Patient's clinical information.

\begin{tabular}{|c|c|c|}
\hline Patient & Age & History \\
\hline 1 & 13 years & Right parietal epileptogenic dysfunction \\
\hline 2 & 14 years & Intractable epilepsy since neonatal period \\
\hline 3 & 17 years & Intractable seizures \\
\hline 4 & 17 years & Intractable seizures \\
\hline 5 & 17 years & $\begin{array}{l}\text { Medically resistant with partial or } \\
\text { temporary control of seizures. }\end{array}$ \\
\hline 6 & 14 years & Left temporal epilepsy with dual pathology \\
\hline 7 & 13 years & Intractable partial seizure \\
\hline 8 & 17 years & Right temporal lobe seizures \\
\hline 9 & 7 years & Jerking left arm and left facial twitching \\
\hline 10 & 15 years & $\begin{array}{l}\text { Cortical dysplacia over right middle frontal } \\
\text { gyrus and gray matter heterotopia over left } \\
\text { occipital peri-atrial region }\end{array}$ \\
\hline 11 & 9 years & Intractable epilepsy \\
\hline 12 & 13 years & Intractable partial epilepsy \\
\hline
\end{tabular}

Twelve children with refractory partial seizures and who underwent pre-surgical evaluation have been considered in this study. The relevant clinical information for all patients is given in Table 1. Since EEG data of these patients were recorded subdurally, this sample of 12 children is considered significant for the research aims of this dissertation. 
It should be noted that although subdural data is assumed throughout this dissertation, the algorithm and support method developed are generalized to accommodate external recordings (i.e., cortical surface) as well.

\subsubsection{RECORDING APPARATUS}
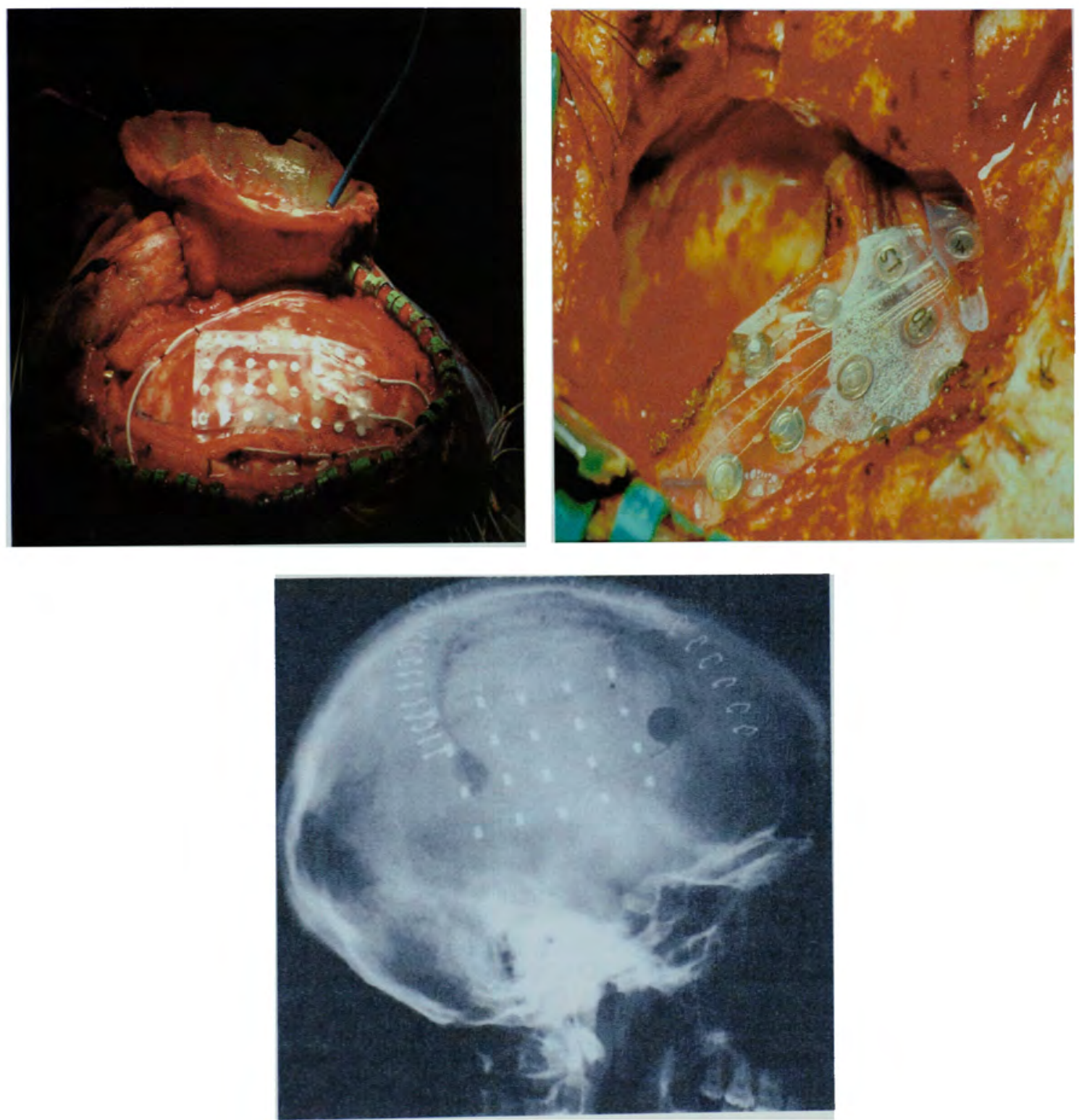

Figure 5.2. Intracranial electrodes as placed during surgery (courtesy of Miami Children's Hospital). 
The subdural EEG data was recorded using XLTEK Neuroworks Ver.3.0.5 (equipment manufactured by Excel Tech Ltd. Ontario, Canada). Sampling frequency of $500 \mathrm{~Hz}$ with $0.1-70 \mathrm{~Hz}$ bandpass filter settings and 12 bits $\mathrm{A} / \mathrm{D}$ conversion were used to obtain the digital EEG recordings. Figure 5.2 shows the intracranial recording strips placed on the brain of one patient for illustrative purposes and to highlight the clinical significance of such a preoperative process.

\subsubsection{DATA ANALYSIS}

Input data in this study was subdural EEG segments of 20 to 3600 seconds duration of epileptic patients. The first step in the procedure involved identifying the pertinent electrodes in the overall interictal EEG recordings in which the seizures occurred. The physicians performed this task initially through visual inspection of the recorded data. A computer program was later developed in order to detect automatically interictal spikes so as to provide more accurate and consistent input data to the proposed classification algorithm (Adjouadi et al., 2004; Cabrerizo et al, 2005).

The interictal activity of five of these patients was localized in distinct regions, while the rest of the patients presented a single epileptic focus. To classify those electrodes that are more likely to lead to an epileptic seizure, a program was developed in order to quantify characterizing patterns inherent to such type of electrodes.

Prior to pre-processing the data, and based on the information provided by the physicians, electrodes leading to seizure were labeled differently from the others such that calculations can be referred to one class or the other. 
The classification algorithm as designed consists of progressive steps as summarized next. The results obtained are then revealed in order to assess both the validity of such steps and the merit of each step for identifying a suitable classifier.

\subsubsection{DEVELOPMENT STEPS OF A NOVEL CLASSIFICATION ALGORITHM}

\section{- A SYNOPSIS}

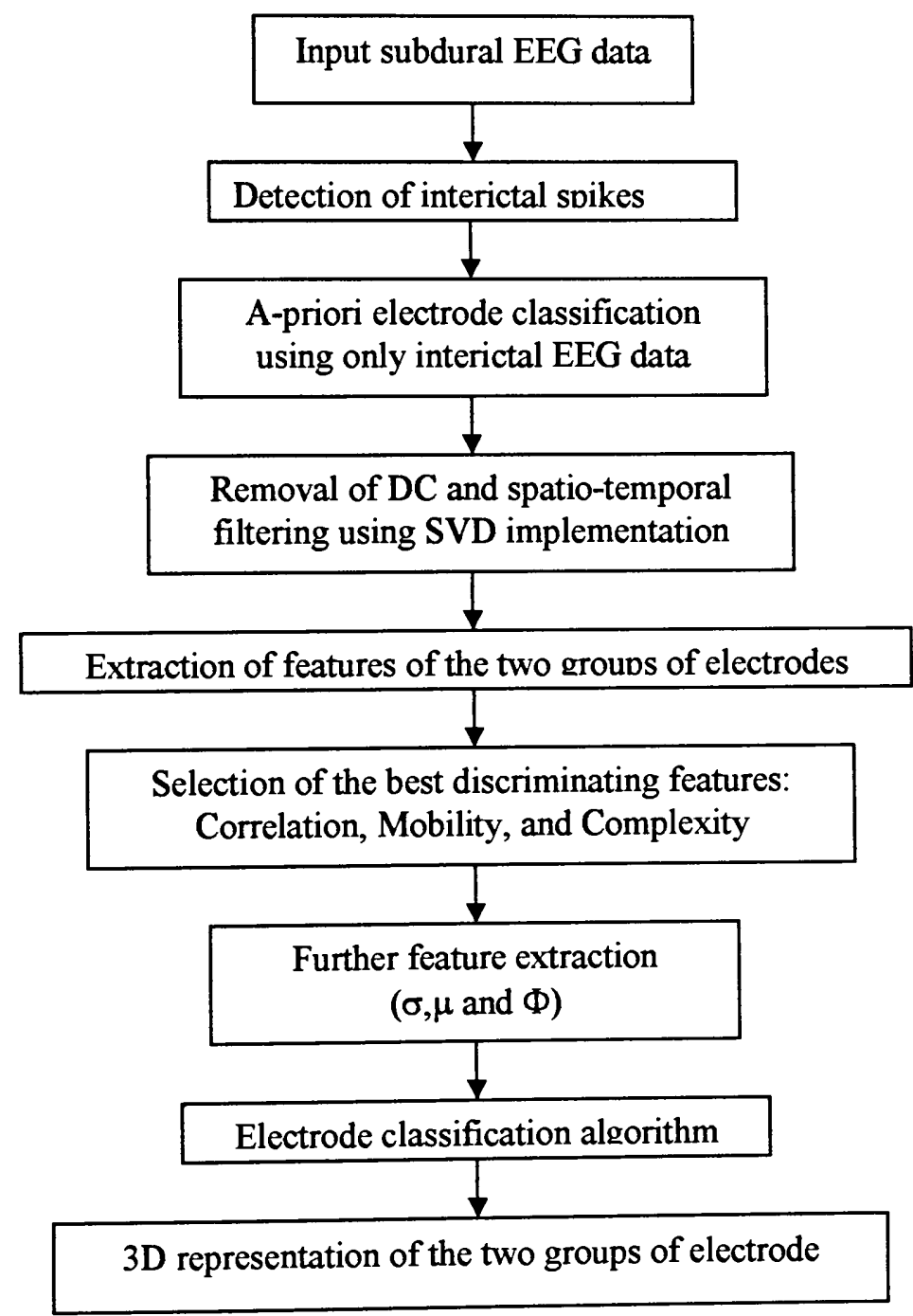

Figure 5.3 Flow chart of the different steps in the EEG data analysis process. 
In order to understand the integrated algorithm that was established in this dissertation for the prescribed goals, the following development steps and a flow chart of tasks are provided as follows.

- Collect subdural EEG data from different patients at Miami Children's Hospital, and perform initial assessments on how the dynamics of these EEG recordings change before, during, and after a seizure. The assumption here is that the onset time of the seizures is presupposed by clinical experience. Recall that the objective here is not to predict seizures but to understand, quantify and classify these EEG dynamics, with the intent to augment the localization mechanisms of epileptic foci and to potentially facilitate the derivation of seizure predictors, given the new knowledge that is elicited.

- Establish new mathematical derivations that accommodate nonlinear dynamics of EEG data and provide quantitative measurements that are inherent to electrodes that lead to seizure in contrast to those that did not.

- Correlate clinical features with the findings from prior steps in order to determine whether the patient has a consistent source of ictal activity, which is coming from the location concerning specific group of channels, and establish relationships between the relative placements of electrodes with the dynamic behavior of EEG signals prior, during and after a seizure.

- Use regression lines as linear approximations of brain dynamics and observe visually among the many discriminating parameters used, which ones led to optimized results across all patients. 
- Automate through neural networks the classification process by determining in a new constructed feature space that was used as input space to the ANN decision functions in order to separate electrodes that lead to seizure from those that do not, prior and after a seizure onset.

- Determine from the knowledge gained from these new findings what the prospects are for developing new methods to seizure prediction.

\subsection{IMPLEMENTATION STEPS}

The implementation steps involved (1) Filtering EEG data, (2) Assessing the EEG Nonlinear dynamics, (3) Implementing regression line approximations, and (4) Computing the Neural Network for linear classification.

\subsubsection{FILTERING THE INPUT EEG DATA}

In this preprocessing step DC and high frequency components were removed from the data using a $0.1-100 \mathrm{~Hz}$ band-pass filter (Soong, 1995). To extract the dominant EEG patterns, the Singular Value Decomposition (SVD), which is based on the eigenvalues decomposition method, was applied, where the first five largest singular values were retained to filter the data and to account for the useful EEG epileptic signal information (Cabrerizo et al., 2005; Zhukov et al., 2000).

When EEG is digitally recorded, it is possible to represent it in a matrix form. In this case the input EEG data matrix I, whose columns correspond to the electrodes and 
whose rows correspond to the time samples, is decomposed into three different matrices as follows:

$$
\mathrm{I}=\mathrm{U} \Sigma \mathrm{V}^{\mathrm{T}} \text { with } \quad \Sigma=\operatorname{diag}\left(\sigma_{\mathrm{i}}\right)
$$

Matrices $\mathrm{U}$ and $\mathrm{V}$ are orthogonal and $\Sigma$ is a diagonal matrix containing singular values in descending order as its elements, and $\mathrm{V}^{\mathrm{T}}$ denotes the transpose of V (Adjouadi et al., 2006; Gonzalez et al., 2000). An orthogonal matrix has the property that the product of the its transpose and the matrix itself yields as a result identity matrix. As shown in Figure 5.4, from the diagonal matrix $\Sigma$, the larger singular values were retained (in this case the first five were deemed sufficient for the analysis), so a better approximation is obtained, or equivalently, more information is contained in that approximation. The remaining singular values were set to zero, thus a new matrix $\Sigma$ was created. To reconstruct the original matrix, only the columns of matrix $U$, rows of matrix $\mathrm{V}^{\mathrm{T}}$ and the new matrix $\Sigma$ corresponding to the quality of approximation are used using equation 5.1. One of the important applications of SVD is for the approximation of the matrices. This approximation is known as rank-reducing approximation, which means that a given matrix can be decomposed into a summation of a number of lower-rank matrices. The approximated matrix $M$, containing the most significant EEG information was used in the subsequent steps to yield the results shown in Figure 5.5.

Each singular value is associated with an orthogonal pattern contained in the data matrix M. Larger singular values correspond to higher pattern correlation with the 
original data. Therefore, SVD allows the extraction of dominant patterns from the recorded EEG data by keeping the largest singular values and setting to zero those with small values assuming that they correspond to noise and other undesired artifacts.

Application of SVD in this fashion has the effect of spatio-temporal filtering, since EEG data is stored in a matrix, whose columns represent electrodes and carry spatial information, and whose rows represent time samples containing temporal information (Barreto, 1993; Mirkovic, et. al., 2003). However, it is not always true to assume that each SVD pattern is generated by a single source, since SVD patterns are mathematically orthogonal, which is not the case with actual biological EEG sources. This implementation concluded the preprocessing step.

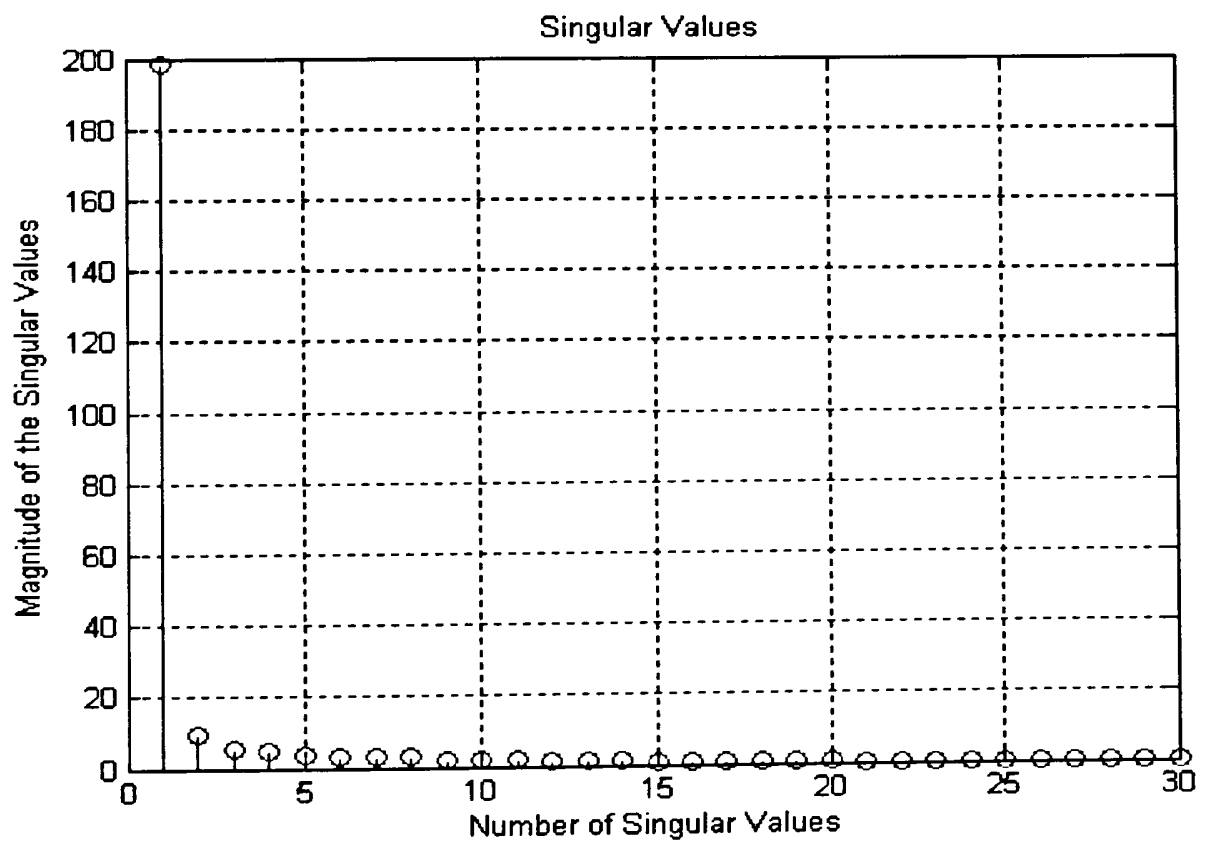

Figure 5.4. Singular values of the diagonal matrix $\Sigma$. 


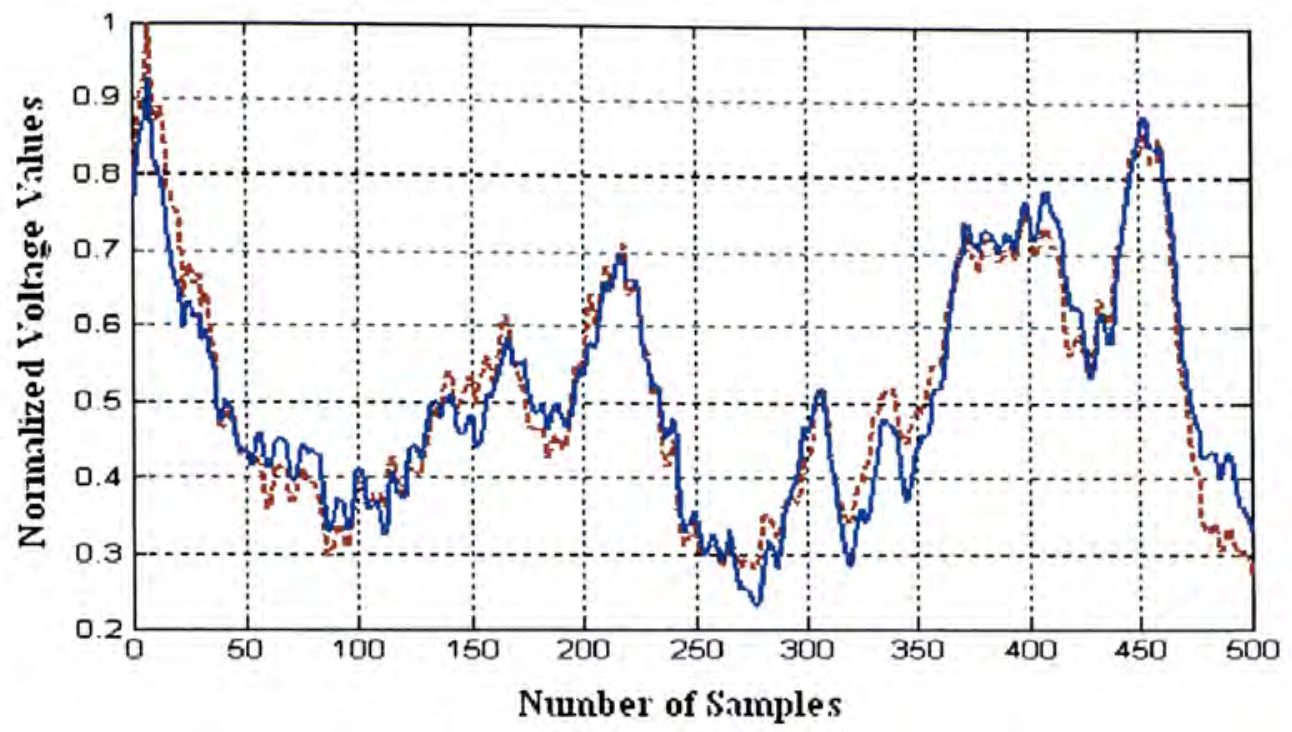

Figure 5.5. SVD decomposition applied to the EEG signal (original signal is shown in red dots and the resulting signal after filtering is shown in continuous blue).

\subsubsection{ASSESSMENT OF THE EEG NONLINEAR DYNAMICS}

In the evaluation of all relevant parameters that could potentially asses the nonlinear dynamics of EEG, it was revealed in Chapter 4, that correlation dimension $(\mathrm{R}(\mathrm{r}))$, Mobility $(\mathrm{M}(\mathrm{y}))$ and Complexity $(\mathrm{C}(\mathrm{y}))$ performed best for the research goals of this dissertation. These parameters were explained in detail in the previous chapter, and recall that equations $4.15,4.16$, and 4.17 were used to calculate them (Geiger, 1978).

The next step dealt with extracting features from the filtered EEG matrix. Figure 5.6 illustrates the clusters dispersion of the two types of electrodes (leading and not leading to seizure) based on the mean and standard deviation of the 3 aforementioned parameters that were implemented. 


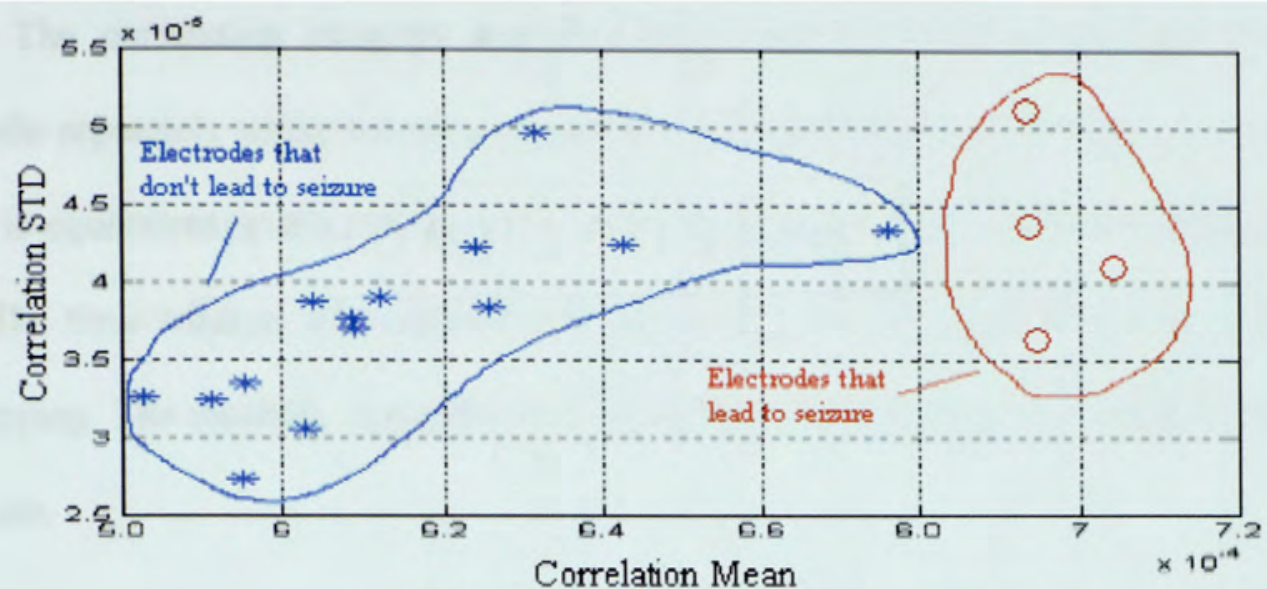

(a) Using Correlation Integral

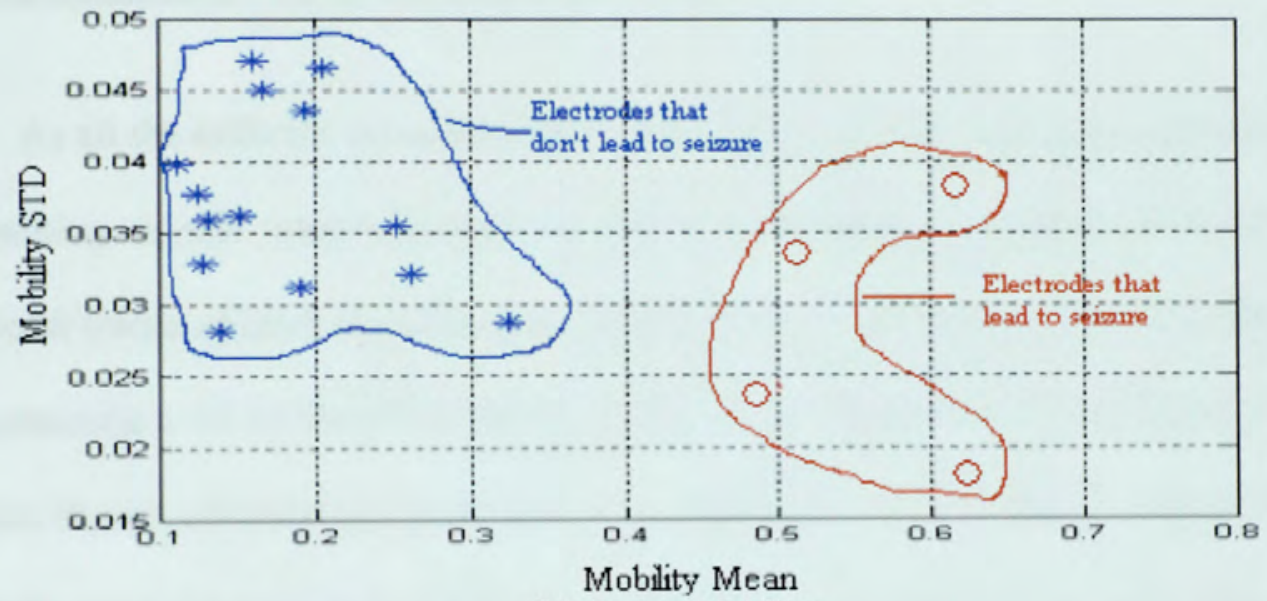

(b) Using Mobility



(c) Using Complexity

Figure 5.6 Clustering of the electrodes based on mean and standard deviation of their correlation values. 
The correlation integral, mobility, and complexity were computed for each electrode separately using successive epochs of non-overlapping windows of 1 second, which is equivalent to the 500 samples sampling rate for all the recorded subdural EEG data. The time window of 1 second was defined across the whole EEG signal without overlapping. The resulting signal for each parameter over time was thus obtained for each electrode.

\subsubsection{REGRESSION LINE APPROXIMATIONS}

As all the different parameters were represented in time, regression lines for all of the resulting signals were estimated, in order to facilitate observation of the different behavioral trends of each electrode with respect to the computed feature. This also helps in determining a linear classifier that separates in the feature versus time space, the two different classes of electrodes (leading and not leading to seizure). One condition to make this study more relevant from a clinical view point was to require from these two classes of electrodes to be totally independent in terms of source location, and synchronicity in spike firing. After obtaining regression lines for all electrodes, the two groups of channels that were to be classified can be assessed in terms of the trend of the behavior of each parameter in time. Regression lines thus provide for an easier way to track the changes that took place in the signals with respect to the parameters analyzed prior to, during, and after an ictal state. 
The mathematics for a regression line, as illustrated in Figure 5.7, is based on deriving the equation of line $\mathrm{Y}^{\prime}$. It is a linear approximation that simply consists of a line such that it approximates the data points with minimum cumulative error.

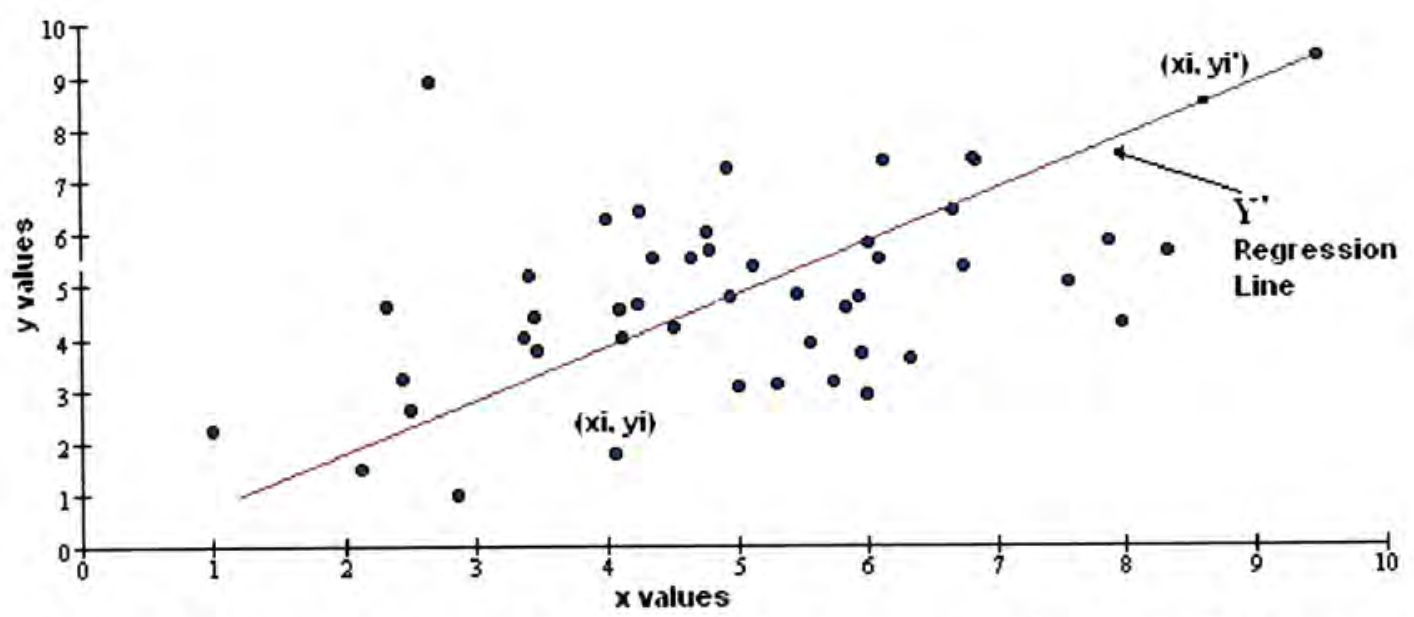

Figure 5.7. Representation of a regression line to represent the trend of the feature.

For each point $\left(x_{i}, y_{i}\right)$, approximation $y_{i}^{\prime}$ is given by $y_{i}^{\prime}=\alpha+\beta x_{i}$, where $\left|y_{i}-y_{i}^{\prime}\right|$

is the error in estimation, that yields a minimized error $E$ given by $E=\sum_{i=1}^{n}\left(y_{i}-y_{i}^{\prime}\right)^{2}$. In these two equations, $y_{i}$ is the value for the actual data point, and $y_{i}^{\prime}$ is its approximation in the $y$-axis. The values $\alpha$ and $\beta$ are calculated as follows:

$$
\alpha=\bar{y}-\beta \bar{x}
$$

with,

$$
\beta=\frac{\sum_{i=1}^{n}\left(x_{i}-\bar{x}\right)\left(y_{i}-\bar{y}\right)}{\sum_{i=1}^{n}\left(x_{i}-\bar{x}\right)^{2}} \text {, where } \bar{x}=\frac{1}{n} \sum_{i=1}^{n} x_{i} \text { and } \bar{y}=\frac{1}{n} \sum_{i=1}^{n} y_{i}
$$


These computed linear approximations were used for each electrode to facilitate visualization of the overall trend of each electrode.

\subsubsection{NEURAL NETWORK STRUCTURE FOR LINEAR CLASSIFICATION}

At this stage, a plot of the three selected features revealed well defined electrodes clusters. No other features produced class clusters so compact and separated from each other. But extrapolation of this mechanism of classification in time did not work as anticipated since the time dynamics of the parameters strongly changed from one recording to the other, despite visible class clustering. In a parameter vs. time plot, the separating points between the two electrode groups changed from one recording to another.

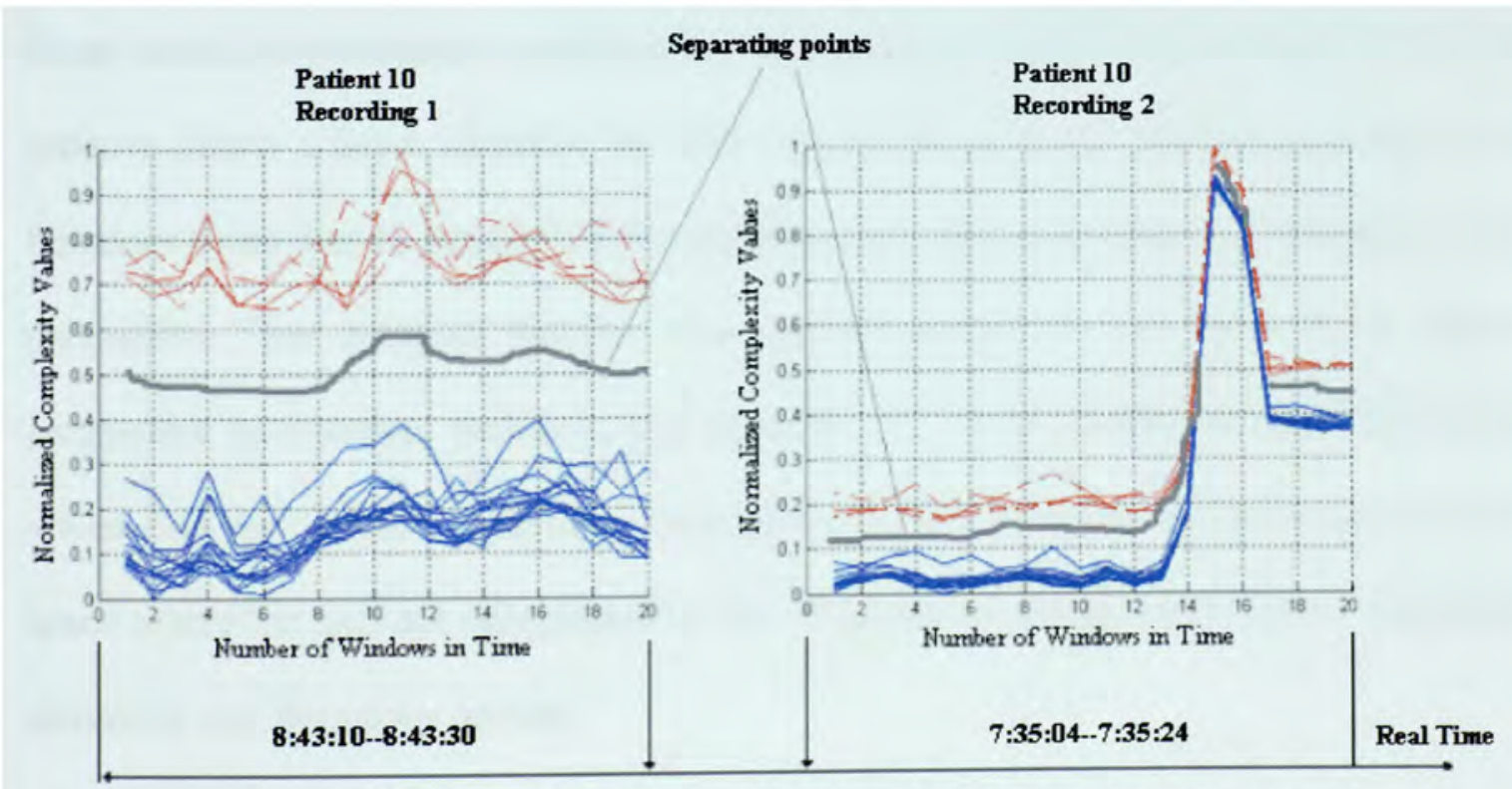

Figure 5.8. Electrode clusters changing their relative location in the feature vs. time plot. 
This is best illustrated in Figure 5.8. Note that each plot is represented only for 20 seconds in two different segments of the EEG data. The real time, where the data was taken, is displayed at the bottom of the two plots.

In order to consider this relative change and yet make real-time classification possible, time independent analysis was performed by computing for each feature three statistical parameters, namely the mean of the regression line that represents the feature behavior, the standard deviation of the parameter over time and the power of the frequency spectrum of the feature over time.

The average and the standard deviation for each regression line were computed for each group of electrodes. Also, the Fourier Transform was applied to the behavior of each parameter over time and its power frequency was calculated for each electrode. These statistical parameters were then inputted to an artificial neural network (ANN) in order to obtain a linear classifier for each feature (Ayala et al., 2004). Linear decision functions could then be established for classifying the electrodes based on these statistical parameters. One decision function was created exclusively for each of the three parameters (correlation, mobility, and complexity). These specific decision functions would find the optimum separating plane between the two classes of electrodes in a 3D space where the axis are represented by the statistical parameters used (mean, standard deviation, and frequency power).

Establishing an artificial neural network (ANN) that is trained to extract seizureleading features of interictal EEG is a significance outcome, since this ANN 
(1) can help to overcome the subjective factor associated with human classification;

(2) can serve as a second expert for decision process validation; and

(3) can be used for fast automated seizure leading channels detection, even for online recordings, sparing EEG technicians the tedious task of long-term monitoring.

The network configuration used in this research is illustrated in Figure 5.9. The 3 input neurons correspond to the mean, standard deviation, and frequency power $(\mu, \sigma, \Phi)$ of the parameter analyzed. The output would be 1 or -1 , which indicates if a given channel leads to seizure or not, respectively.

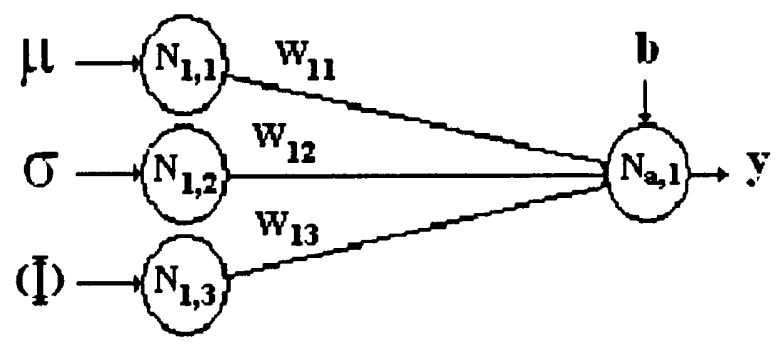

Figure 5.9. Network configuration.

A percentage of misclassification errors were calculated for each patient so that more reliable decisions can be made with the help of clinical expertise. In order to account for any experienced overlap, classification performance was evaluated using Receiver Operating Characteristics (ROC) analysis.

The classifiers are three decision functions of the form:

$$
f_{\xi}(\underline{X})=w_{1} \cdot \mu_{\xi}(\underline{X})+w_{2} \cdot \sigma_{\xi}(\underline{X})+w_{3} \cdot \Phi_{\xi}(\underline{X})+w_{4}
$$


The subscript $\xi$ is defined as follows: $\quad \xi= \begin{cases}R & \text { for Correlation } \\ M & \text { for Mobility } \\ C & \text { for Complexity }\end{cases}$

Where $\underline{X}$ is a vector containing the values of the specific parameter (correlation integral, complexity, or mobility) for all time windows; $w_{1}, w_{2}, w_{3}$, and $w_{4}$ are coefficients and $\mu(\underline{\mathrm{X}}), \sigma(\underline{\mathrm{X}})$, and $\phi(\underline{\mathrm{X}})$ are the mean, the standard deviation, and the frequency power of vector $\underline{X}$, respectively. Electrodes are classified as leading to seizure only if $f_{\xi}(\underline{X})>0$ for a specific feature.

The decision functions consisted of feed-forward ANNs trained via backpropagation. These ANNs are structured with 3 input neurons and 1 output neuron, with linear activation functions. This type of structure produces a linear classifier as illustrated in Figure 5.10.


(a) 2-D Plot

(b) 3-D Plot

Figure 5.10. Electrode representation in the feature space. 
In this figure, the electrodes are plotted according to the values of 3 statistical parameters (the standard deviation, mean, frequency power) computed from one specific parameter (for example, the mobility) during a certain time period. This type of classification extends to higher dimensions and higher order of complexity if necessary. The results of this dissertation indicate that planar surfaces in a 3D plot (mean, standard deviation, and frequency) are sufficient for this type of classification.

In order to avoid network memorization, training was performed on a randomly selected recording (data segment \#1) of a patient with electrodes properly labeled as leading to seizure or not, while the remaining recordings were kept for testing purposes.

The mathematical basis for the backpropagation algorithm is an optimization technique known as gradient descent, in which the aim is to minimize an error function $\mathrm{E}$. For this to be accomplished, the error gradient $\frac{\partial E}{\partial w}$ with respect to the variable under optimization (in this case, weight $\mathrm{w}$ ) is set to zero:

$$
\frac{\partial E}{\partial w}=0
$$

When training an ANN to approximate a given input-output relationship based on a set of training patterns, as it is the case here, obtaining optimum weights for each pattern does not make any sense, since the optimum values would vary from pattern to pattern. Instead, the solution is found by computing average weights. Thus, it is more convenient to look for $\Delta \mathrm{w}$ and then iteratively update the weights based on small 
proportions of $\Delta \mathrm{w}$. When an optimum is found, both gradient and $\Delta \mathrm{w}$ should be zero, such that $w_{\text {new }}=w_{\text {old }}$. Then, the amount of weight update $(\Delta \mathrm{w})$ can be simply set to a portion of the negative gradient of the error function E, i.e.

$$
\Delta \mathrm{w}=-\mathrm{c} \cdot \frac{\partial \mathrm{E}}{\partial \mathrm{w}}
$$

and the weight update can be set to

$$
\mathrm{w}_{\text {new }}=\mathrm{w}_{\text {old }}+\Delta \mathrm{w}
$$

which allows obtaining the optimum weight via successive approximations using simple numeric techniques (Eberhart, 1990; Ko, 2000).

In equation 5.6, $\mathrm{C}$ is a constant (called learning rate) that is used to control the speed of the convergence, and $\mathrm{E}$ is the error made through the approximation, which is the square difference between its output $y$ and its target $t$ :

$$
E=\frac{1}{2}(y-t)^{2}
$$

The $\frac{1}{2}$ is a convenience factor for canceling derivations of square errors.

The derivation of the learning rule for a weight $w$ is precisely based on the minimization of this gradient. In general, the update formula for a weight between two neurons depends on the following factors: (1) on the layer the two neurons belongs to; (2) 
on the value of the signal being transmitted through the weight; and (3) on possible connections outgoing from the second neuron as illustrated in Figure 5.11:

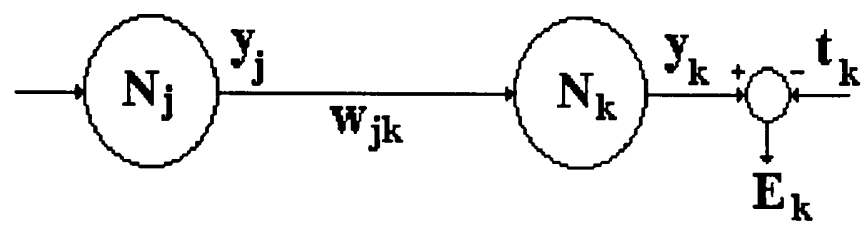

Figure 5.11. Representation of a weight $w_{j k}$ between two neurons $N_{j}$ and $N_{k}$, showing neuron outputs, targets and error.

In general, for any weight $\mathrm{w}_{\mathrm{jk}}$ going from neuron $\mathrm{N}_{\mathrm{j}}$ to neuron $\mathrm{N}_{\mathrm{k}}$, as shown in Figure 5.9, the weight update formula is given by:

$$
\Delta w_{j k}=-\alpha \frac{\partial E_{k}}{\partial y_{k}} \phi_{k}{ }^{\prime}\left(x_{k}\right) y_{j}
$$

Where $\phi_{\mathrm{k}}{ }^{\prime}\left(\mathrm{x}_{\mathrm{k}}\right)$ is the first derivative of the transfer function of neuron $\mathrm{N}_{\mathrm{k}}, y_{j}$ is the output of neuron $\mathrm{N}_{\mathrm{j}}$, and $\mathrm{x}_{\mathrm{k}}$ is the inner activation of this neuron, i.e., the sum of all incoming signals. The value of the gradient $\frac{\partial E_{k}}{\partial y_{k}}$ depends on the layer the neuron is placed. For the last layer neurons, the weight update formula is simple; nevertheless, for the other neurons, the formula contains a weighted average of previously computed gradients of neurons of next layers as shown in (5.10): 


$$
\frac{\partial E_{k}}{\partial y_{k}}=\left\{\begin{array}{lr}
y_{k}-t_{k} & \text { for the last layer } \\
\sum_{m=1}^{M} w_{k m} \phi_{m}^{\prime}\left(x_{m}\right) \frac{\partial E_{m}}{\partial y_{m}} & \text { for otherlayers }
\end{array}\right.
$$

In (5.10), $M$ is the number of neurons in the layer after neuron $N_{k}$ and $m$ is the index for all of these neurons.

The algorithm is called backpropagation because the errors are backpropagated from the last layer back to the first one in order to compute a new (better) set of weights. This operation is done several times on the data base containing the training patterns until a stop condition is found. The influence a neuron output has to the total net error is obtained by using the chain rule from that neuron to the next layer (Hellman, 1999).

\subsection{ANALYSIS OF THE RESULTS}

The algorithm was implemented for all 12 patients, but only the results of 8 patients were taken into consideration to analyze the misclassification percentage of the linear classifier. When applying the algorithm to the other 4 patients, the classification results were not as good as expected due to a high overlap. Upon more clinical inquiry, it was revealed that such situations do indeed happen when electrodes presenting interictal activity are situated in the same region and the interictal spikes are happening simultaneously, which was the case for these 4 patients. 
This is an important finding in that when electrodes belong to a same region, there is evidence of cross-talk between electrodes (even synchronization at times) yielding similar behaviors of these electrodes whether they lead to seizure or not. This finding will have significance in the pre-assessment process in developing seizure prediction algorithms to avoid potential misinterpretations of the results whether it is for prediction or for classification.

Results of the 8 patients indicate that the proposed EEG analysis allows for the defining of two regions of electrodes, one for electrodes leading to an ictal state and another for the remaining electrodes that do not lead to such a state. Also, using key discriminating parameters, characterization of the behavior of the interictal EEG over time is made possible. It was found that the mobility is greater for those electrodes that lead to seizure. Since mobility deals with the first derivative and the first derivative is the rate of change of the signal's voltage amplitude. Furthermore, the steeper the slope of the EEG signal, the greater the amplitude of the first derivative. In addition, results indicate that the dynamics of complexity is higher for those channels that go into an ictal state, meaning that there are more sharp fluctuations in those signals.

The false positive rate (FPr) indicates the misclassification percentage of the number of electrodes that do not lead to a seizure and is calculated as:

$$
\mathrm{FPr}=\frac{\mathrm{FP}}{\text { Negativelnstances }}=\frac{\mathrm{FP}}{\mathrm{TN}+\mathrm{FP}}
$$


The false negative rate $(\mathrm{FNr})$ is the misclassification percentage of the number of channels that are leading to seizure and it is calculated as:

$$
\mathrm{FNr}=\frac{\mathrm{FN}}{\text { Positive Ins tan ces }}=\frac{\mathrm{FP}}{\mathrm{FN}+\mathrm{TP}}
$$

The weights of the decision functions, for every parameter implemented, obtained for classifying the electrodes on patient 1 are given in Table 2 for illustrative purposes. Similar weights are computed for each patient.

Table 5.2. Weights of the decision functions for each parameter for patient 1 .

\begin{tabular}{|c|c|c|c|c|}
\hline Function & W1 & W2 & W3 & W4 \\
\hline Correlation & 13.444123 & -1.969053 & -2.520771 & -0.278356 \\
\hline Mobility & 5.782254 & 7.892331 & -3.078757 & -1.812404 \\
\hline Complexity & 6.819882 & 8.265253 & -3.536731 & -1.962363 \\
\hline
\end{tabular}

Based on the data used to find the classifiers, confusion matrices were computed for each of the 8 subjects and the 3 classifiers that were obtained (correlation integral, mobility, and complexity) in order to obtain the degree of accuracy of the overall classification process. The rate of missed detections as well as the rate of incorrect positive detections is given in Table 5.3.

As it can be observed from Table 5.3, the results are much better for complexity than for the other two parameters. The misclassification percentage rates are calculated 
for the group of electrodes leading to seizure $(\mathrm{FNr})$ and for to the group of electrodes that do not lead to seizure (FPr).

Table 5.3. Percentage of misclassification.

Note: Results have been averaged across all EEG segments.

\begin{tabular}{|c|c|c|c|c|c|c|}
\hline PATIENT & $\begin{array}{c}\text { Correlation } \\
\text { FNr }\end{array}$ & $\begin{array}{c}\text { Correlation } \\
\text { FPr }\end{array}$ & $\begin{array}{c}\text { Mobility } \\
\text { FNr }\end{array}$ & $\begin{array}{c}\text { Mobility } \\
\text { FPr }\end{array}$ & $\begin{array}{c}\text { Complexity } \\
\text { FNr }\end{array}$ & $\begin{array}{c}\text { Complexity } \\
\text { FPr }\end{array}$ \\
\hline Patient 1 & $40.7 \%$ & $0.0 \%$ & $14.3 \%$ & $12.9 \%$ & $14.3 \%$ & $0.0 \%$ \\
\hline Patient 2 & $37.5 \%$ & $0.0 \%$ & $20.0 \%$ & $0.0 \%$ & $0.0 \%$ & $0.0 \%$ \\
\hline Patient 3 & $0.0 \%$ & $8.2 \%$ & $0.0 \%$ & $0.0 \%$ & $0.0 \%$ & $0.0 \%$ \\
\hline Patient 4 & $33.3 \%$ & $0.0 \%$ & $0.0 \%$ & $0.0 \%$ & $0.0 \%$ & $0.0 \%$ \\
\hline Patient 5 & $0.0 \%$ & $5.0 \%$ & $0.0 \%$ & $0.0 \%$ & $0.0 \%$ & $0.0 \%$ \\
\hline Patient 6 & $28.6 \%$ & $0.0 \%$ & $14.3 \%$ & $0.0 \%$ & $14.3 \%$ & $0.0 \%$ \\
\hline Patient 7 & $42.8 \%$ & $0.0 \%$ & $28.6 \%$ & $0.0 \%$ & $14.3 \%$ & $0.0 \%$ \\
\hline Patient 8 & $42.8 \%$ & $5.0 \%$ & $14.3 \%$ & $0.0 \%$ & $0.0 \%$ & $0.0 \%$ \\
\hline
\end{tabular}

Key findings can be affirmed as follows: (1) it was found that at any window of time along the EEG signal (independent of time), acceptable classifiers could be obtained using just the complexity values; (2) A search for such decision functions across patients is ineffectual, because experiments reveal that such decision functions are patient dependent; (3) It is extremely important that when one is to search for such decision functions, electrodes should be analyzed only if they are localized in different locations and with recorded interictal spikes not happening simultaneously.

As an example, Figures 5.10 and 5.11 show a plot of complexity over time for one of the EEG data segments of patients 1 and 10. The red color represents electrodes leading to seizure and the blue color represents those that do not. It is evident that complexity is higher for those channels that go into an ictal state. There is more activity in those channels, meaning that the amplitude of the EEG signal is higher in those 
electrodes. Then, the regression lines for each channel are represented as well, in order to depict the trend of that specific parameter for each electrode. A 3-D representation of the parameters using their statistical features is also shown. These values were then inputted to the neural network implemented with the purpose of finding a suitable classifier for each feature. As can be observed, for patient 10 as an example, the separation between the two groups is succinct, so a linear classifier will be more reliable than the one obtained for the case of patient 1 .

A simultaneous look at the two patient's data as illustrated in Figures 5.12 and 5.13 reveals that significant overlap still exits in patient 1 in both $2 \mathrm{D}$ and $3 \mathrm{D}$ planes although less significant in the 3D plane. Therefore, a decision was made to perform intra patient analysis as means to discriminate the two electrode classes for this specific patient. Though these decisions, further experimentations reveal that the electrodes leading to seizure show a remarkable behavior that may lead to the development of predictive measures.

At this point of the study, it was noted that the only recordings that presented the most overlap (not a clear separation between the two groups of electrodes) were those of patient 1 . Observing a clear separation in the feature plots for the rest of the patients, linear electrode classifier for them are developed using the same ANN architecture as for patient 1 , except that the convergence rate is much faster and where the classification results are $100 \%$ accurate given the clear separability of the clusters. 

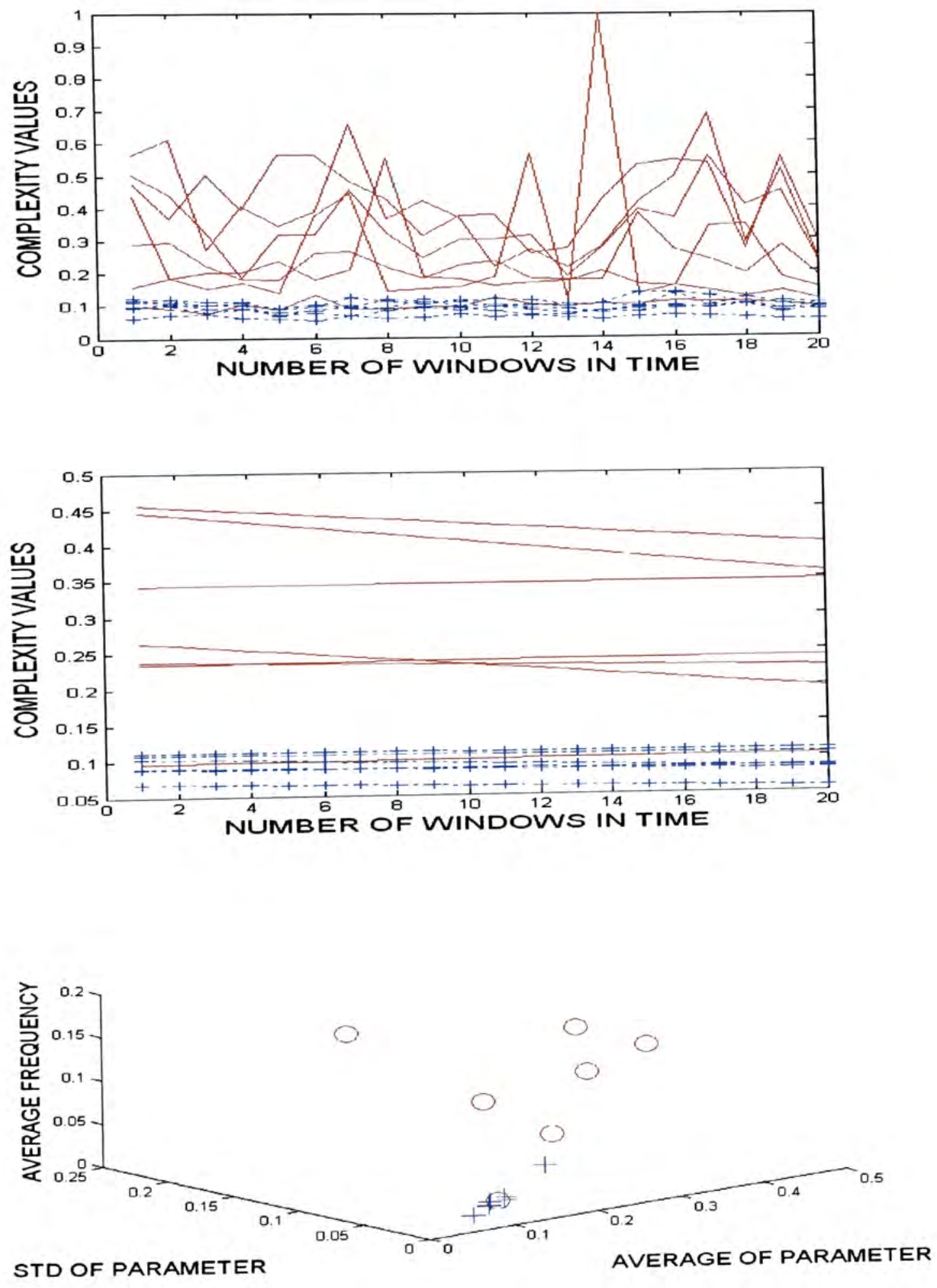

Figure 5.12. Complexity results for patient 1 (case with overlapping). 

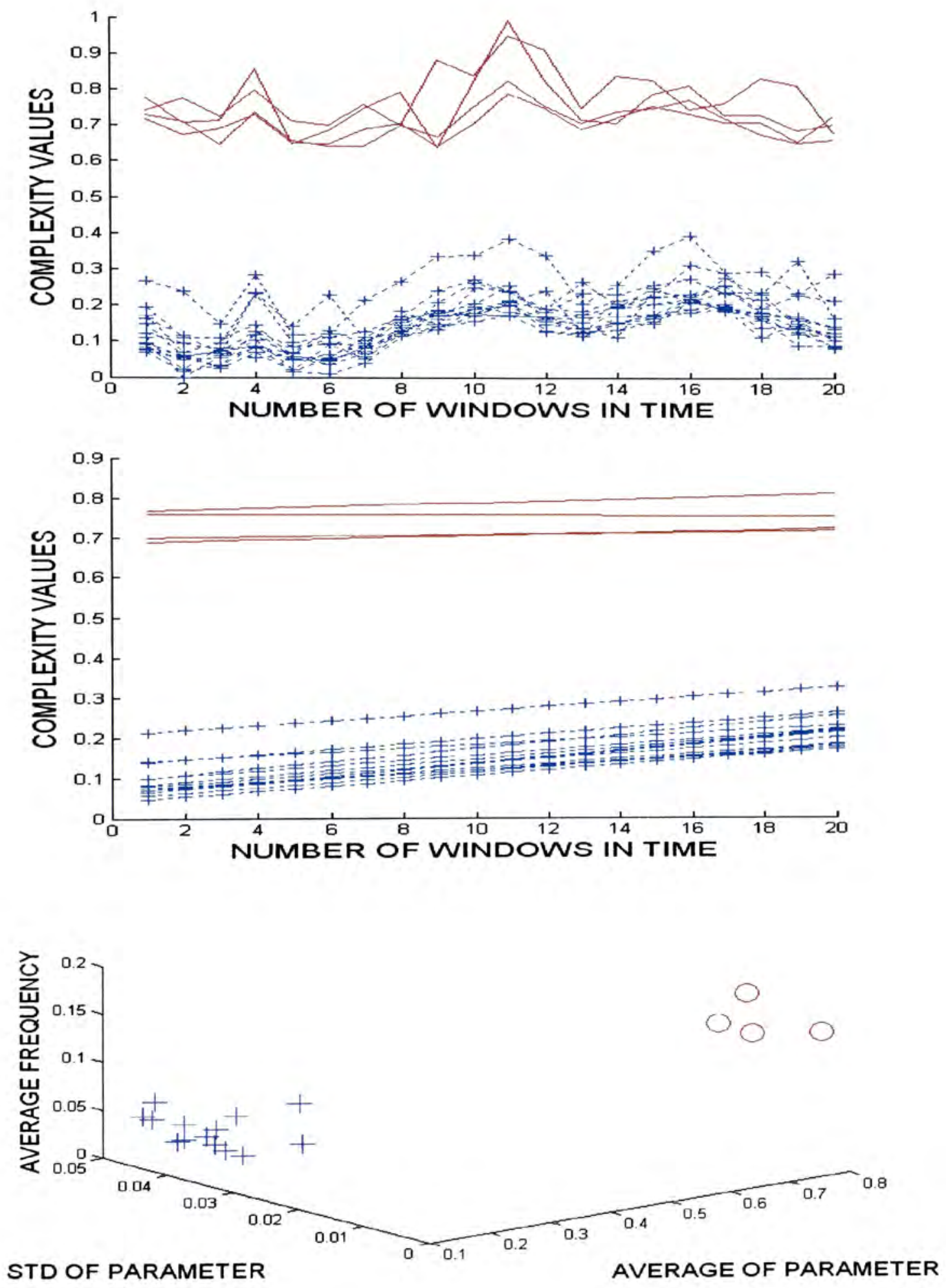

Figure 5.13. Complexity results for patient 10 (case with no overlapping). 
Making the ANN converges and yielding accurate classification results should be emphasized as well as that the separability is achieved because of the choices of the 3 discriminant features of mean, standard deviation, and frequency power. This in itself constitutes a mayor contribution of this dissertation.

To evaluate the performance of the classifiers, an evaluation was performed on test data (data not used to train them), but from which it was known which electrodes lead to seizure and which did not. The disagreements between the actual classification and the predicted classification were analyzed using the following terms:

- TRUE POSITIVE (TP): An electrode leading to seizure is identified as such by the algorithm.

- FALSE POSITIVE (FP): An electrode not leading to seizure is not identified as such by the algorithm.

- FALSE NEGATIVE $(F N)$ : An electrode leading to seizure is not identified as such by the algorithm.

- TRUE NEGATIVE (TN): An electrode not leading to seizure is identified as such by the algorithm.

Consequently, the standard measures of sensitivity and precision (Eberhart, 1990; Gonzalez, 1993) are used for the evaluation of the algorithm:

- Sensitivity $(S)$ is defined as " the likelihood that an event will be detected given that it is present": 


$$
\mathrm{S}=\frac{\mathrm{TP}}{\mathrm{TP}+\mathrm{FN}}
$$

- Precision $(P)$ or positive predictive value is "the likelihood that a signal of an event is associated with the event, given that a signal occurred":

$$
P=\frac{T P}{T P+F P}
$$

Using all these terms, a confusion matrix is created for each data segment in order to calculate the misclassification errors for all files belonging to the same patient.

Actual $-/+$ signifies the electrodes that actually belong to the negative/positive class (don't lead/lead to seizure), and

Predicted $-/+$ stands for the electrodes that are classified by the algorithm as negative/positive class.

Each entry in the matrix represents one specific type of error or assertion. For example, the value in the intersection of Actual - and Predicted + , is the amount of electrodes that the program erroneously detected as leading to seizure. It is represented as FP (false positive) or also FP rate.

Table 5.4. Confusion matrix and meaning of its entries.

\begin{tabular}{|l|c|c|}
\hline & Predicted - & Predicted + \\
\hline Actual - & TN & FP \\
\hline Actual + & FN & TP \\
\hline
\end{tabular}


Applying ROC analysis, the performance of the classifiers obtained for each feature of patient 1 used for illustrative purposes followed these steps:

- A complete intra-patient analysis was performed using different segments of EEG.

- Each classifier was trained for a base segment and tested in the rest of the segments.

- Confusion matrices were created for each file and the FP and TP rates were also calculated for each time window.

Misclassification error $\left(E_{\mathcal{M}}\right)$ is computed as the ratio of the positive and negative errors (FP and FN, or misclassification) and the total number of electrodes (or correct classification instances) as shown in Equation 18:

$$
E_{M}=\frac{F P+F N}{\mathrm{FP}+\mathrm{FN}+\mathrm{TP}+\mathrm{TN}}
$$

For patient 1, the total number of classification instances (electrodes) was 14 with 7 positive and 7 negative instances (i.e. 7 electrodes leading to seizure and 7 not leading to seizure).

Given the poor classification results obtained particularly for patient 1, a detailed analysis was conducted on the corresponding EEG data. The results of this analysis are shown in the next section. 


\subsubsection{RESULTS OF CASE WITH OVERLAPPING (PATIENT \# 1)}

All classifiers were first evaluated using the original training data as well as using unused data segments form the same patient recordings. Tables 5.5 through 5.10 show the performance of the classifier for the three different parameters recorded from patient 1 . Table 5.5 shows the misclassification errors obtained for electrode classification based on the complexity measure. A high misclassification average rate of $33.33 \%$ makes this classifier not so reliable. Details of how misclassification occurred in the different recordings of the same patient are depicted in Table 5.6. Based on the confusion matrix of Table 5.4, Tables 5.6, 5.8, and 5.10 are structured for every parameter.

Table 5.7 illustrates the performance of the correlation-based classifier. Here, a high misclassification average rate $(25.00 \%)$ was obtained. Confusion matrix values are provided in Table 5.8. Similarly, Table 5.9 shows unacceptable misclassification errors (23.81\%) when using mobility. Details of the misclassification rates are shown in Table 5.10 .

Table 5.5. Performance evaluation for classification based on complexity for patient 1 , showing an average misclassification error of $33.33 \%$.

\begin{tabular}{|c|c|c|c|c|}
\hline $\begin{array}{c}\text { Data } \\
\text { Segment }\end{array}$ & FP & FN & $\begin{array}{c}\text { Total } \\
\text { Errors }\end{array}$ & $\begin{array}{c}\text { Misclassification } \\
\text { Error for } \\
\text { Complexity }\end{array}$ \\
\hline $\mathbf{1}$ (base) & 1 & 1 & 2 & 0.14 \\
\hline $\mathbf{2}$ & 3 & 1 & 4 & 0.28 \\
\hline $\mathbf{3}$ & 4 & 0 & 4 & 0.28 \\
\hline $\mathbf{4}$ & 1 & 5 & 6 & 0.43 \\
\hline $\mathbf{5}$ & 1 & 5 & 6 & 0.43 \\
\hline $\mathbf{6}$ & 1 & 5 & 6 & 0.43 \\
\hline
\end{tabular}


Table 5.6. Confusion matrices for all EEG segments of worst case scenario (Patient 1) based on complexity.

\begin{tabular}{|c|c|c|c|c|c|}
\hline $\begin{array}{c}\text { Data } \\
\text { Segment }\end{array}$ & Complexity & Predicted - & Predicted + & FPr & TPr \\
\hline 1 (base) & & & & 0.1429 & 0.8571 \\
\hline & Actual - & 6 & 1 & & \\
\hline & Actual + & 1 & 6 & & \\
\hline $\mathbf{2}$ & & & & 0.4286 & 0.5714 \\
\hline & Actual - & 6 & 1 & & \\
\hline & Actual + & 3 & 4 & & \\
\hline $\mathbf{3}$ & & & & 0.5714 & 0.4286 \\
\hline & Actual - & 7 & 0 & & \\
\hline & Actual + & 4 & 3 & & \\
\hline $\mathbf{4}$ & & & & 0.1429 & 0.8571 \\
\hline & Actual - & 2 & 5 & & \\
\hline & Actual + & 1 & 6 & & \\
\hline $\mathbf{5}$ & & & & 0.1429 & 0.8571 \\
\hline & Actual - & 2 & 5 & & \\
\hline & Actual + & 1 & 6 & & \\
\hline $\mathbf{6}$ & & & & 0.1429 & 0.8571 \\
\hline & Actual - & 2 & 5 & & \\
\hline & Actual + & 1 & 6 & & \\
\hline
\end{tabular}

Table 5.7. Performance evaluation for classification based on correlation for patient 1 , showing an average misclassification error of $25.00 \%$.

\begin{tabular}{|c|c|c|c|c|}
\hline $\begin{array}{c}\text { Data } \\
\text { Segment }\end{array}$ & FP & FN & $\begin{array}{c}\text { Total } \\
\text { Errors }\end{array}$ & $\begin{array}{c}\text { Misclassification } \\
\text { Error for } \\
\text { Correlation }\end{array}$ \\
\hline 1 (base) & 2 & 0 & 2 & 0.14 \\
\hline 2 & 4 & 0 & 4 & 0.28 \\
\hline 3 & 2 & 3 & 5 & 0.36 \\
\hline 4 & 1 & 1 & 2 & 0.14 \\
\hline 5 & 4 & 1 & 5 & 0.36 \\
\hline 6 & 2 & 1 & 3 & 0.21 \\
\hline
\end{tabular}


Table 5.8. Confusion matrices for all EEG segments of worst case scenario (Patient 1) based on correlation.

\begin{tabular}{|c|c|c|c|c|c|}
\hline $\begin{array}{c}\text { Data } \\
\text { Segment }\end{array}$ & Correlation & Predicted - & Predicted + & FPr & TPr \\
\hline $\mathbf{1}$ (base) & & & & 0.2857 & 0.7143 \\
\hline & Actual - & 7 & 0 & & \\
\hline & Actual + & 2 & 5 & & \\
\hline $\mathbf{2}$ & & & & 0.5714 & 0.4286 \\
\hline & Actual - & 7 & 0 & & \\
\hline & Actual + & 4 & 3 & & \\
\hline $\mathbf{3}$ & & & & 0.2857 & 0.7143 \\
\hline & Actual - & 4 & 3 & & \\
\hline & Actual + & 2 & 5 & & \\
\hline $\mathbf{4}$ & & & & 0.1429 & 0.8571 \\
\hline & Actual - & 6 & 1 & & \\
\hline & Actual + & 1 & 6 & & \\
\hline $\mathbf{5}$ & & & & 0.5714 & 0.4286 \\
\hline & Actual - & 6 & 1 & & \\
\hline & Actual + & 4 & 3 & & \\
\hline $\mathbf{6}$ & & & & 0.2857 & 0.7143 \\
\hline & Actual - & 6 & 1 & & \\
\hline & Actual + & 2 & 5 & & \\
\hline
\end{tabular}

Table 5.9. Performance evaluation for classification based on mobility for worst case scenario (patient 1), showing an average misclassification error of $23.81 \%$.

\begin{tabular}{|c|c|c|c|c|}
\hline $\begin{array}{c}\text { Data } \\
\text { Segment }\end{array}$ & FP & FN & $\begin{array}{c}\text { Total } \\
\text { Errors }\end{array}$ & $\begin{array}{c}\text { Misclassification } \\
\text { Error for Mobility }\end{array}$ \\
\hline $\mathbf{1}$ (base) & 1 & 1 & 2 & 0.14 \\
\hline $\mathbf{2}$ & 1 & 1 & 2 & 0.14 \\
\hline $\mathbf{3}$ & 4 & 0 & 4 & 0.28 \\
\hline $\mathbf{4}$ & 4 & 0 & 4 & 0.28 \\
\hline $\mathbf{5}$ & 1 & 3 & 4 & 0.28 \\
\hline $\mathbf{6}$ & 1 & 3 & 4 & 0.28 \\
\hline
\end{tabular}


Table 5.10. Confusion matrices for all EEG segments of worst case scenario (Patient 1) based on mobility.

\begin{tabular}{|c|c|c|c|c|c|}
\hline $\begin{array}{c}\text { Data } \\
\text { Segment }\end{array}$ & Mobility & Predicted - & Predicted + & FPr & TPr \\
\hline 1 (base) & & & & 0.1429 & 0.8571 \\
\hline & Actual - & 6 & 1 & & \\
\hline & Actual + & 1 & 6 & & \\
\hline $\mathbf{2}$ & & & & 0.1429 & 0.8571 \\
\hline & Actual - & 6 & 1 & & \\
\hline & Actual + & 1 & 6 & & \\
\hline $\mathbf{3}$ & & & & 0.5714 & 0.4286 \\
\hline & Actual - & 7 & 0 & & \\
\hline & Actual + & 4 & 3 & & \\
\hline $\mathbf{4}$ & & & & 0.5714 & 0.4286 \\
\hline & Actual - & 7 & 0 & & \\
\hline & Actual + & 4 & 3 & & \\
\hline $\mathbf{5}$ & & & & 0.1429 & 0.8571 \\
\hline & Actual - & 4 & 3 & & \\
\hline & Actual + & 1 & 6 & & \\
\hline $\mathbf{6}$ & & & & 0.1429 & 0.8571 \\
\hline & Actual - & 4 & 3 & & \\
\hline & Actual + & 1 & 6 & & \\
\hline
\end{tabular}

The sensitivity and precision percentages are provided in Table 5.11 for each data segment. The highest average sensitivity percentage was obtained using the complexity parameter and the highest precision percentage was obtained using the correlation parameter.

Figure 5.14 represents a compilation of the performance of all electrode classifiers for patient 1 . It can be observed that for this specific patient, that mobility has the lowest misclassification rate, meaning that mobility turned out to be the best feature to 
discriminate between the two groups of electrodes (leading vs. not leading to seizure). For this patient, the superiority of the correlation and mobility measures is remarkable.

An example of a completely different outcome is given in Figure 5.15. Here, the complexity measure of patient 4 is plotted, showing a very clear separation between the two electrode classes. As mentioned earlier, the same situation was found for the rest of the patients as illustrated in Figures 5.17 through 5.22, which allows to note that the three features $(\mu, \sigma, \Phi)$ have great potential for classifying electrodes leading to seizure, regardless on what type of classifier used with respect to the 3 parameters. Notice that in Figure 5.17, for one of the patients, there is one overlap, which later revealed that the EEG data was taken very close to the seizure onset, and there is always a tendency of the EEG signal just prior to an ictal state to behave in a similar mode for all the electrodes. In other words all electrodes converge into a point where similar behavior is noted just prior to a seizure.

Table 5.11. Sensitivity (S) and precision (P) values case with overlapping (patient 1).

\begin{tabular}{|c|rc|rc|rc|}
\hline $\begin{array}{c}\text { Data } \\
\text { Segment }\end{array}$ & \multicolumn{2}{|c|}{ Correlation } & \multicolumn{2}{|c|}{ Complexity } & \multicolumn{2}{|c|}{ Mobility } \\
\hline & S (\%) & $\mathbf{P ~ ( \% ) ~}$ & $\mathbf{S ~ ( \% )}$ & $\mathbf{P ~ ( \% ) ~}$ & S (\%) & P (\%) \\
\hline $\mathbf{1}$ & 71 & 100 & 86 & 86 & 86 & 86 \\
\hline $\mathbf{2}$ & 43 & 100 & 57 & 80 & 86 & 86 \\
\hline $\mathbf{3}$ & 71 & 63 & 43 & 100 & 43 & 100 \\
\hline $\mathbf{4}$ & 86 & 86 & 86 & 55 & 43 & 100 \\
\hline $\mathbf{5}$ & 43 & 75 & 86 & 55 & 86 & 67 \\
\hline $\mathbf{6}$ & 71 & 83 & 86 & 55 & 86 & 67 \\
\hline Average & 64 & 84 & 74 & 71 & 71 & 84 \\
\hline
\end{tabular}




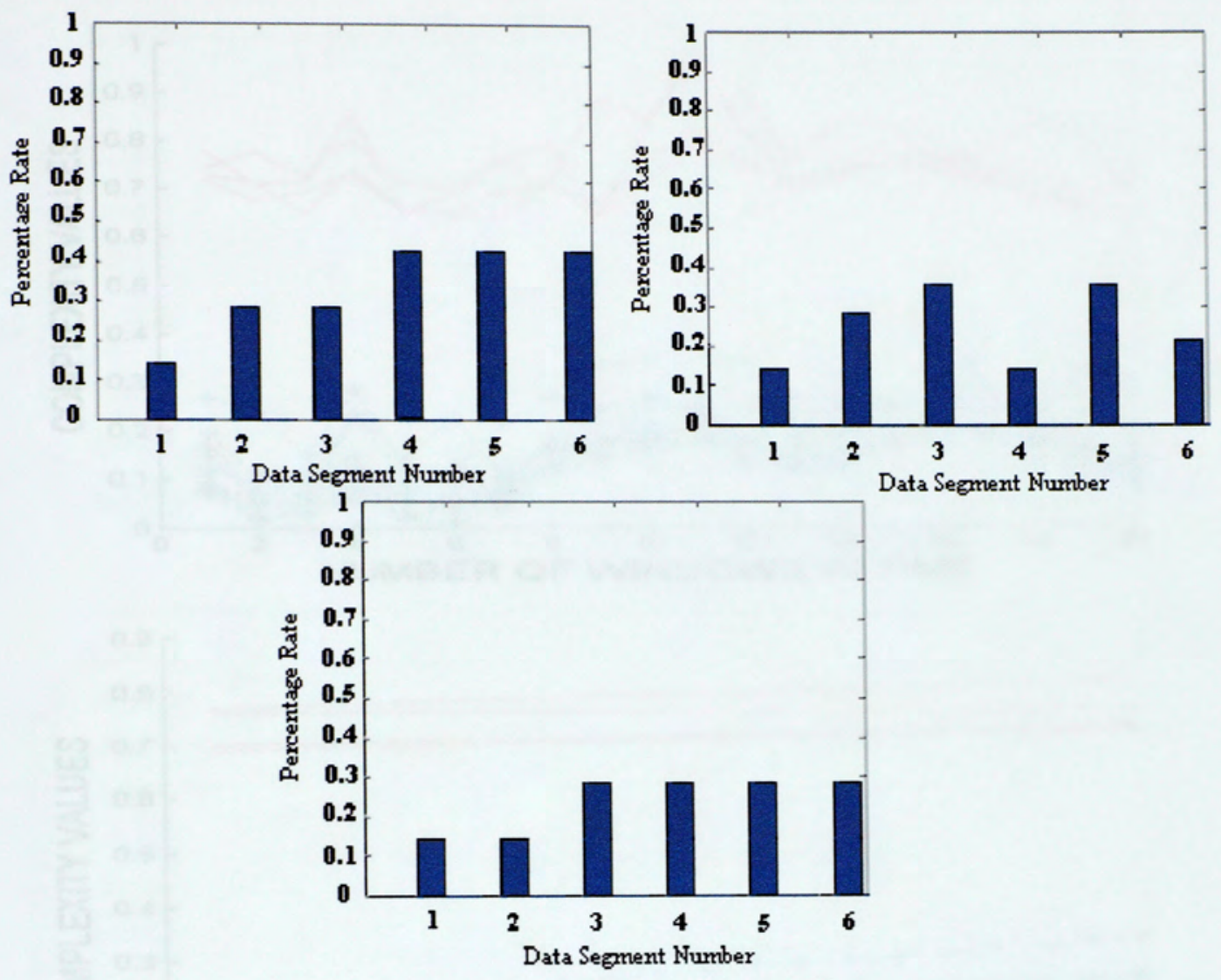

(a) Misclassification Rate of Complexity, Correlation, and Mobility for each data segment.

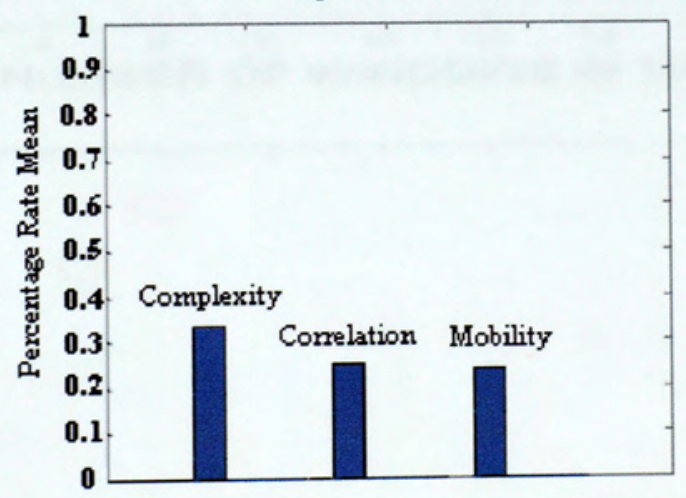

(b) Contrasting misclassification rate of the 3 different parameters.

Figure 5.14. Misclassification rates for the 3 classifiers of case with overlapping (patient 1). 

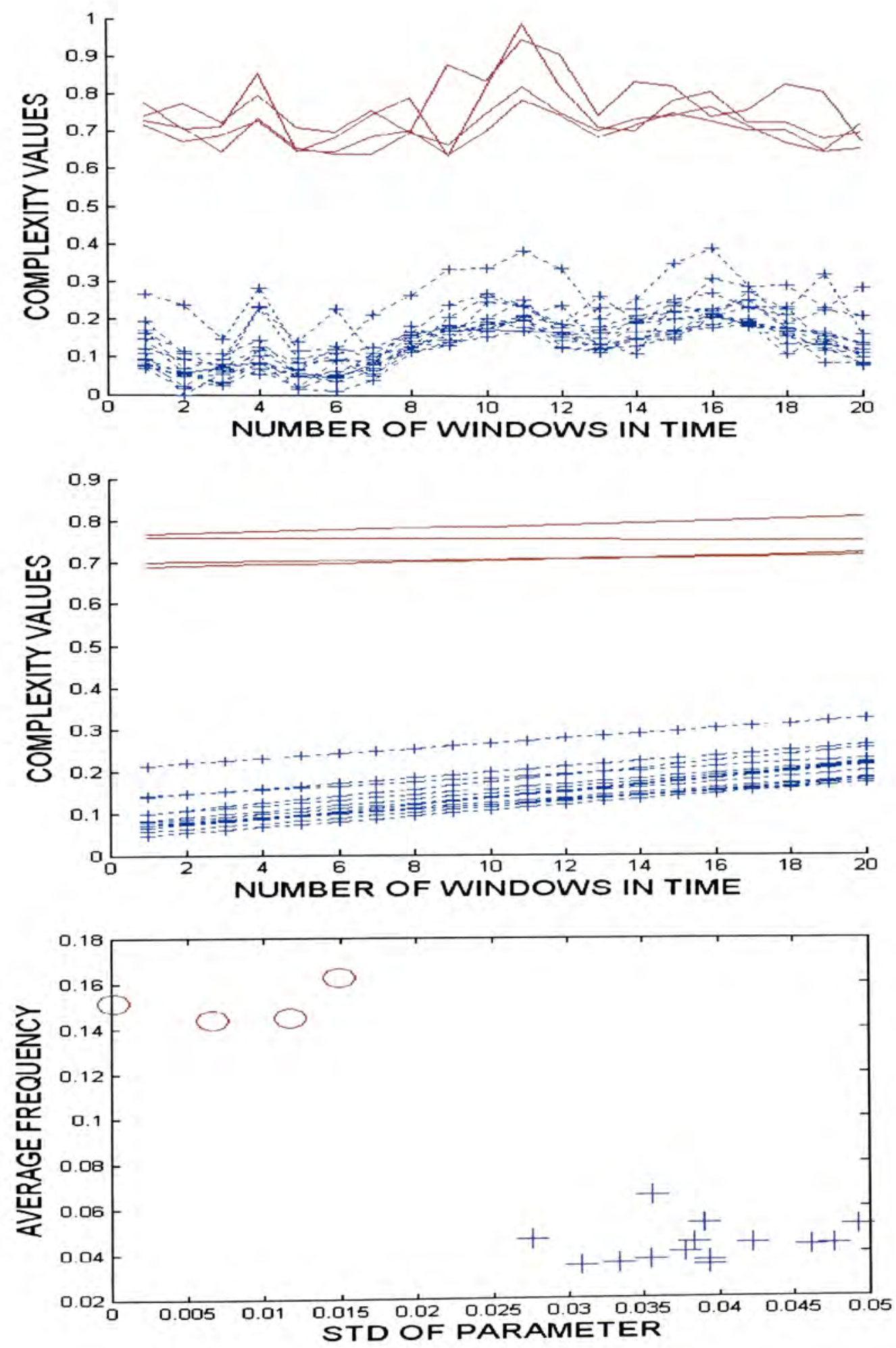

(a) Behavior of parameter, regression lines, average vs. of parameter. 

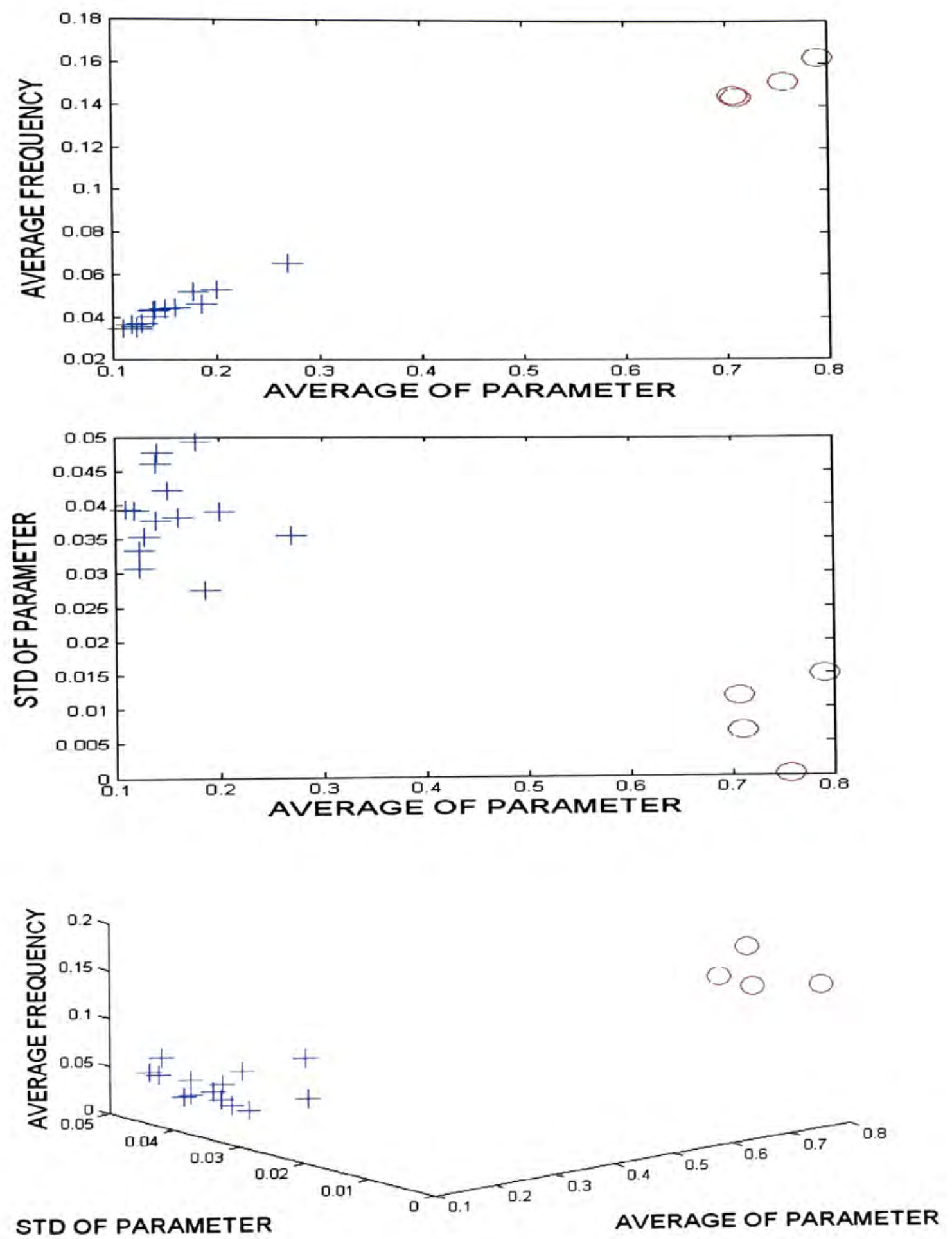

(b) Avg. freq. vs. parameter, std vs. avg. of parameter, avg. freq. vs. parameter. Figure 5.15. Complexity results for patient 4. Note: Leading to seizure (red (o) or red $(-))$ and not leading to seizure (blue $(+)$ or blue $(+-)$ ). 

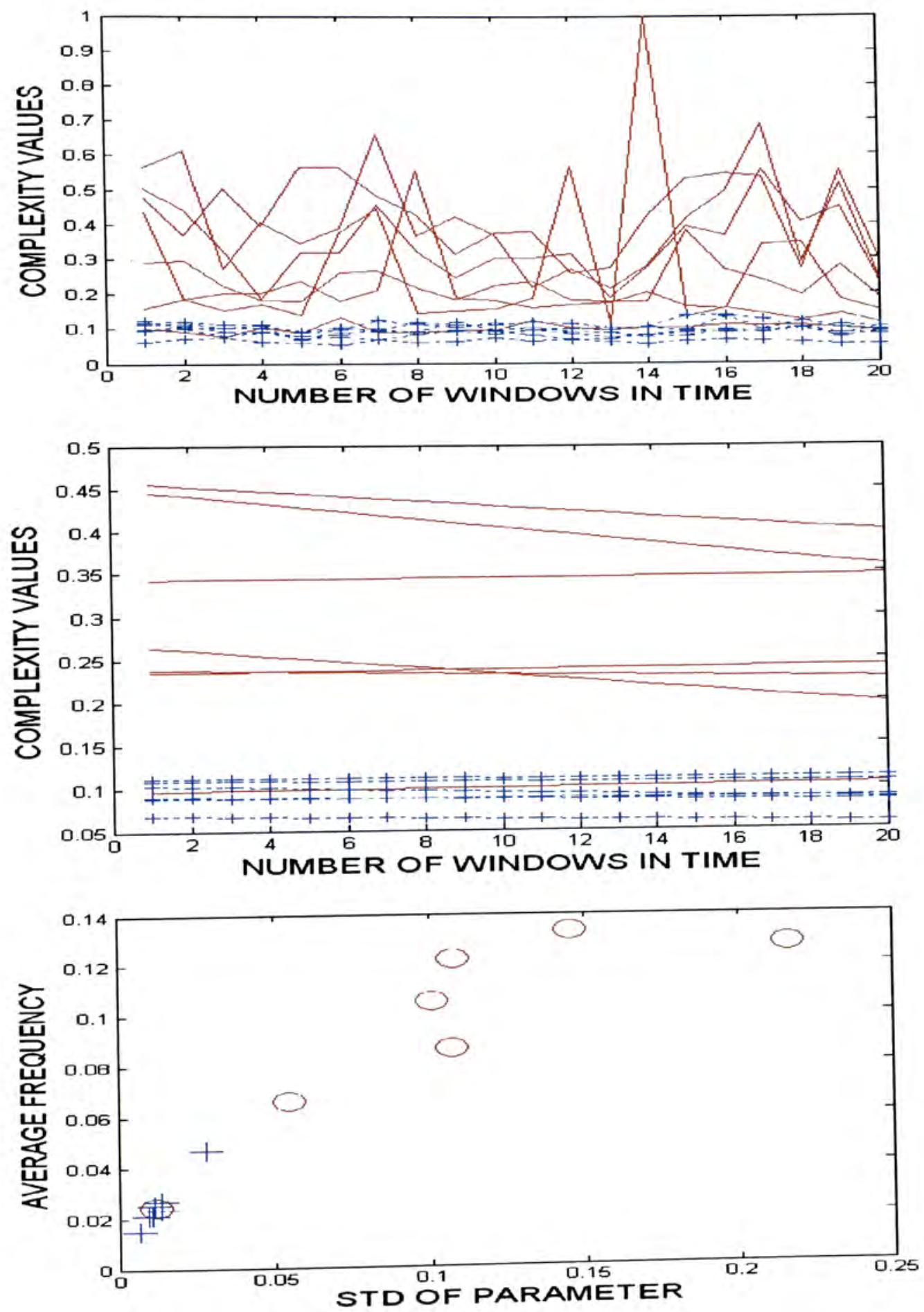

(a) Behavior of parameter, regression lines, average vs. of parameter. 

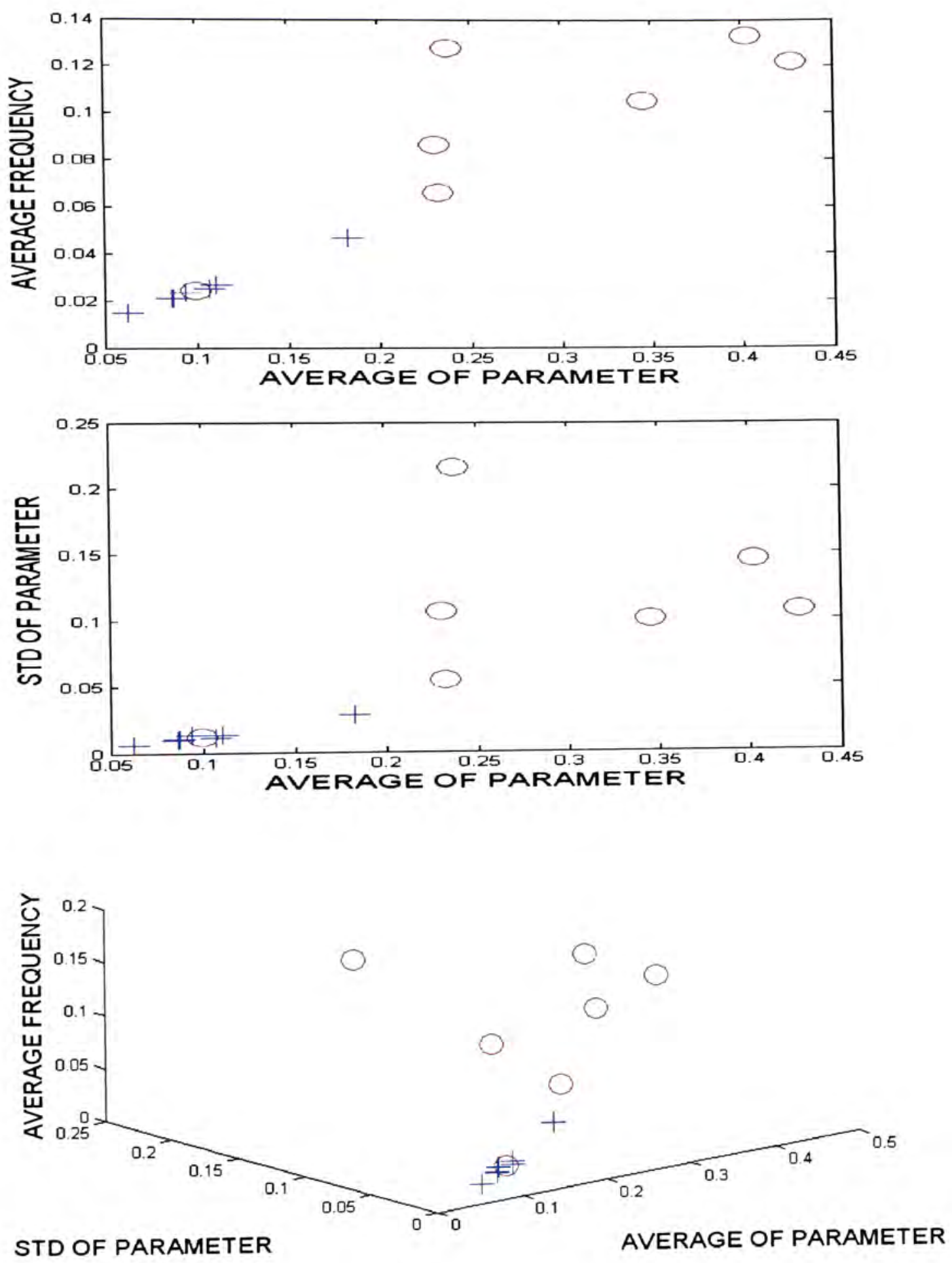

(b) Avg. freq. vs. parameter, std vs. avg. of parameter, avg. freq. vs. parameter.

Figure 5.16. Complexity results for patient 1 (case with overlapping). Note: Leading to seizure (red (o) or red (-)) and not leading to seizure (blue $(+)$ or blue $(+-)$ ). 

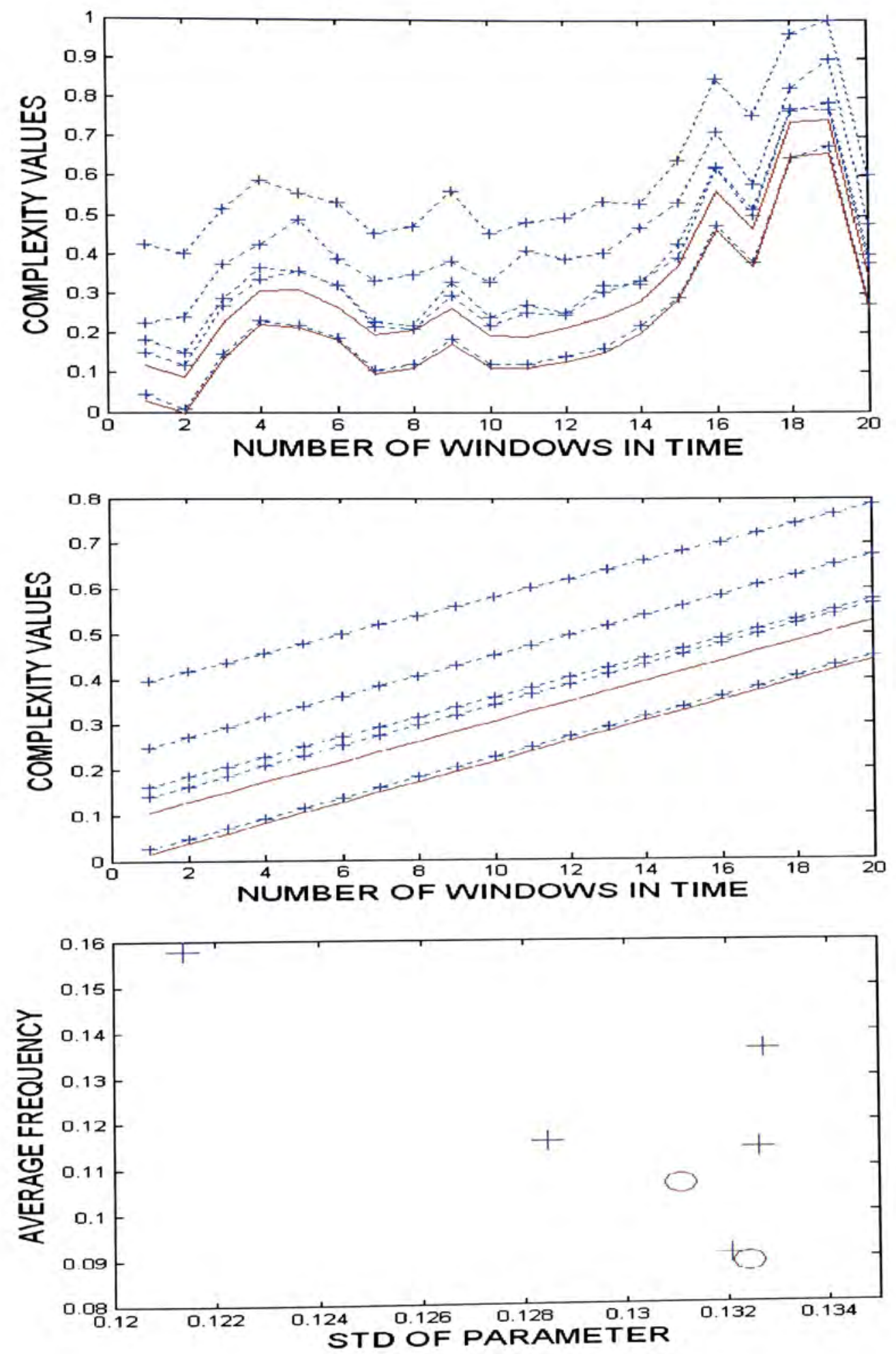

(a) Behavior of parameter, regression lines, average vs. of parameter. 

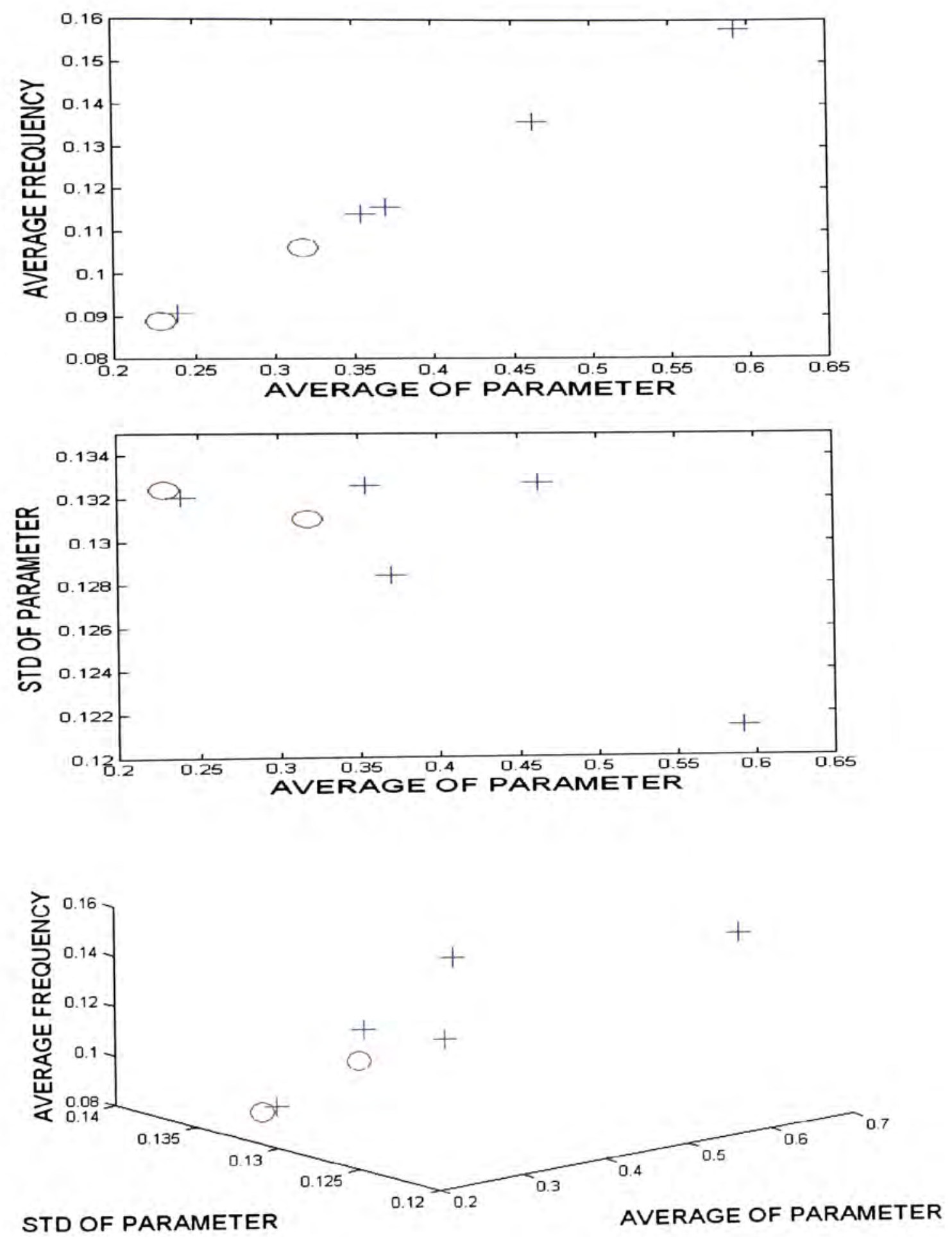

(b) Avg. freq. vs. parameter, std vs. avg. of parameter, avg. freq. vs. parameter.

Figure 5.17. Complexity results for patient 3 . Note: Leading to seizure ( red (o) or red $(-))$ and not leading to seizure (blue $(+)$ or blue $(+-)$ ). 

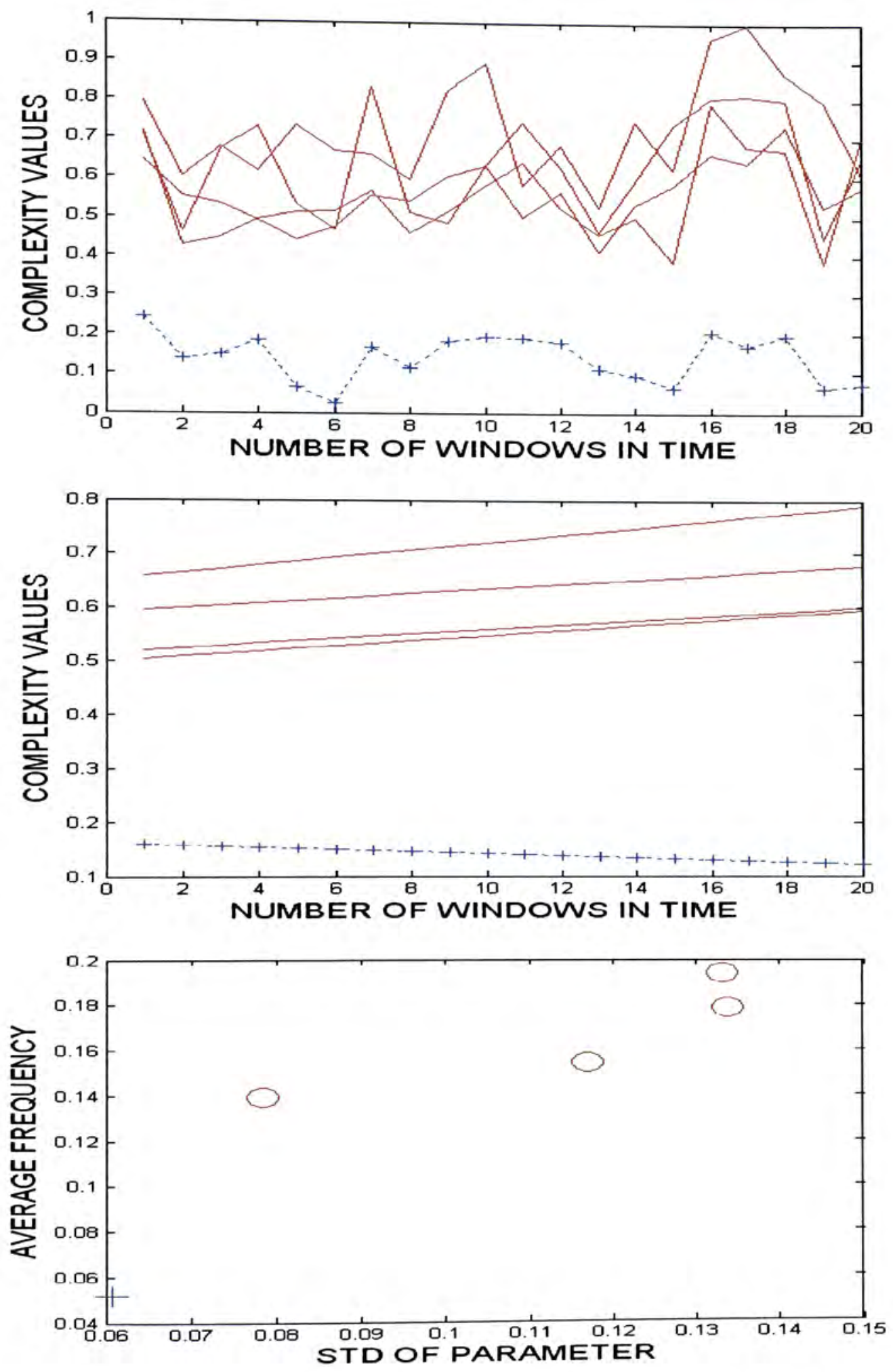

(a) Behavior of parameter, regression lines, average vs. of parameter. 

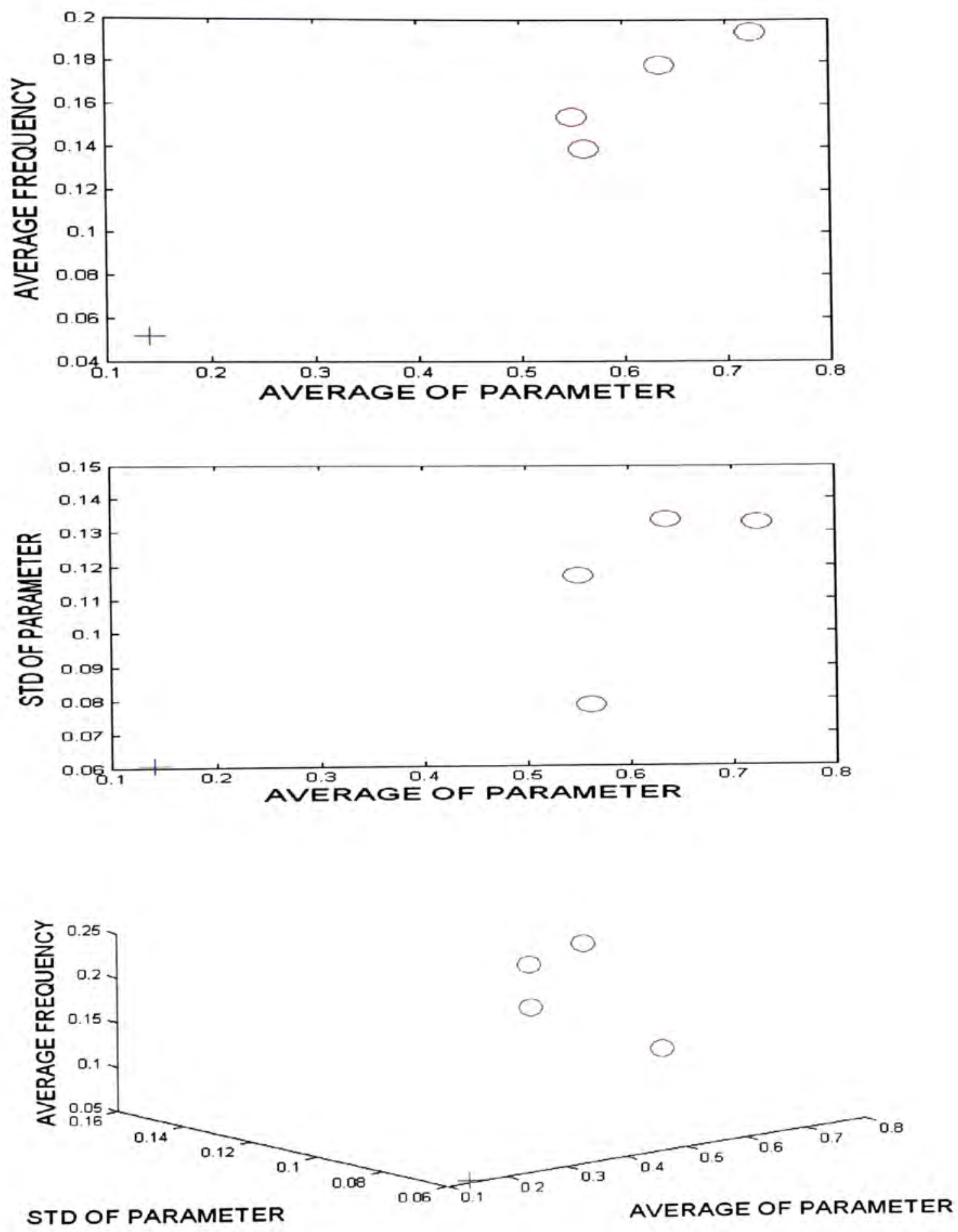

(b) Avg. freq. vs. parameter, std vs. avg. of parameter, avg. freq. vs. parameter.

Figure 5.18 Complexity results for patient 2. Note: Leading to seizure ( red (o) or red $(-))$ and not leading to seizure (blue $(+)$ or blue $(+-)$ ). 



(a) Behavior of parameter, regression lines, average vs. of parameter. 

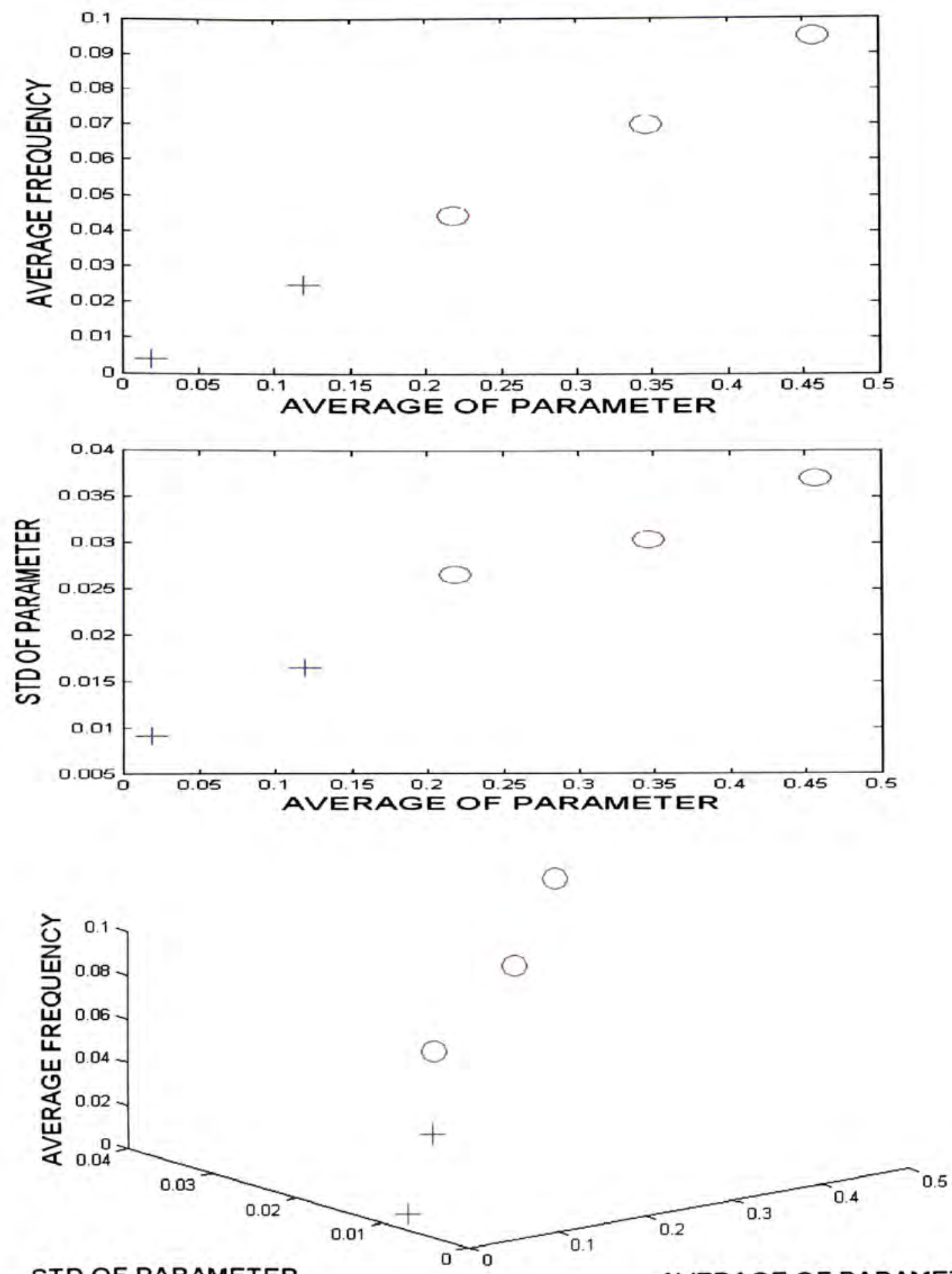

STD OF PARAMETER

AVERAGE OF PARAMETER

(b) Avg. freq. vs. parameter, std vs. avg. of parameter, avg. freq. vs. parameter. Figure 5.19. Complexity results for patient 5 . Note: Leading to seizure (red (o) or red (-)) and not leading to seizure (blue $(+)$ or blue $(+-)$ ). 



(a) Behavior of parameter, regression lines, average vs. of parameter. 

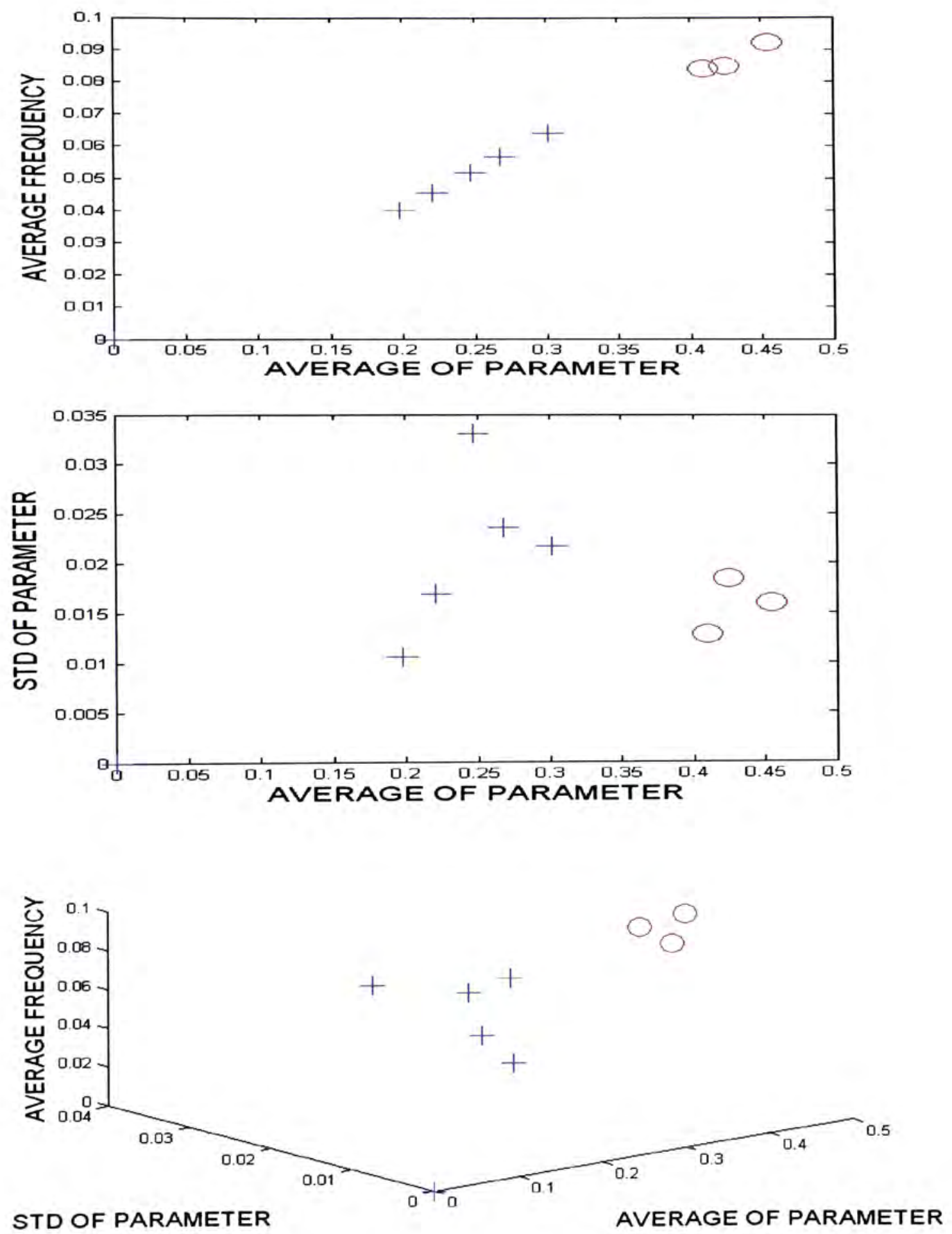

(b) Avg. freq. vs. parameter, std vs. avg. of parameter, avg. freq. vs. parameter. Figure 5.20 Complexity results for patient 6. Note: Leading to seizure (red (o) or red (-)) and not leading to seizure (blue $(+)$ or blue $(+-))$. 

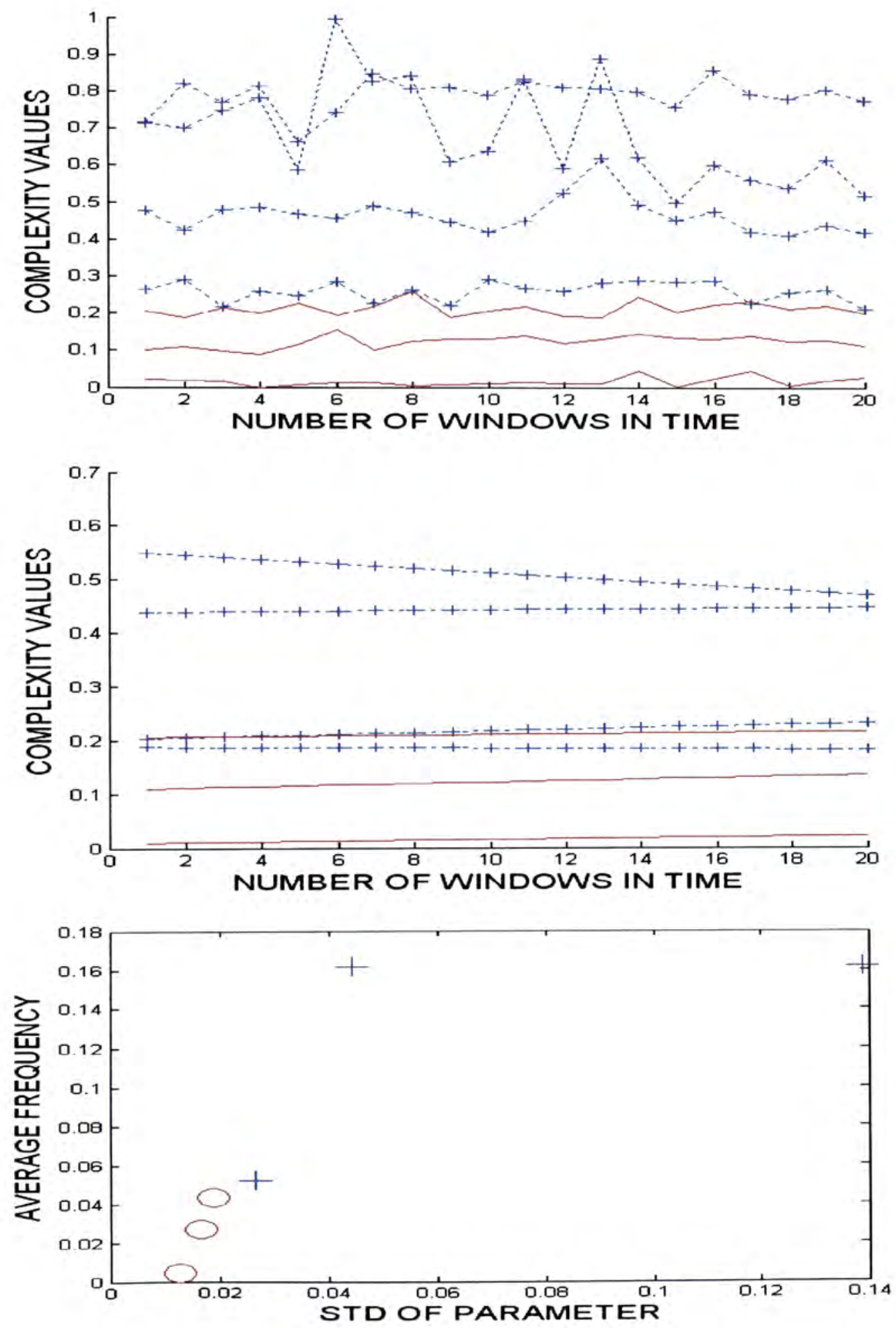

(a) Behavior of parameter, regression lines, average vs. of parameter. 

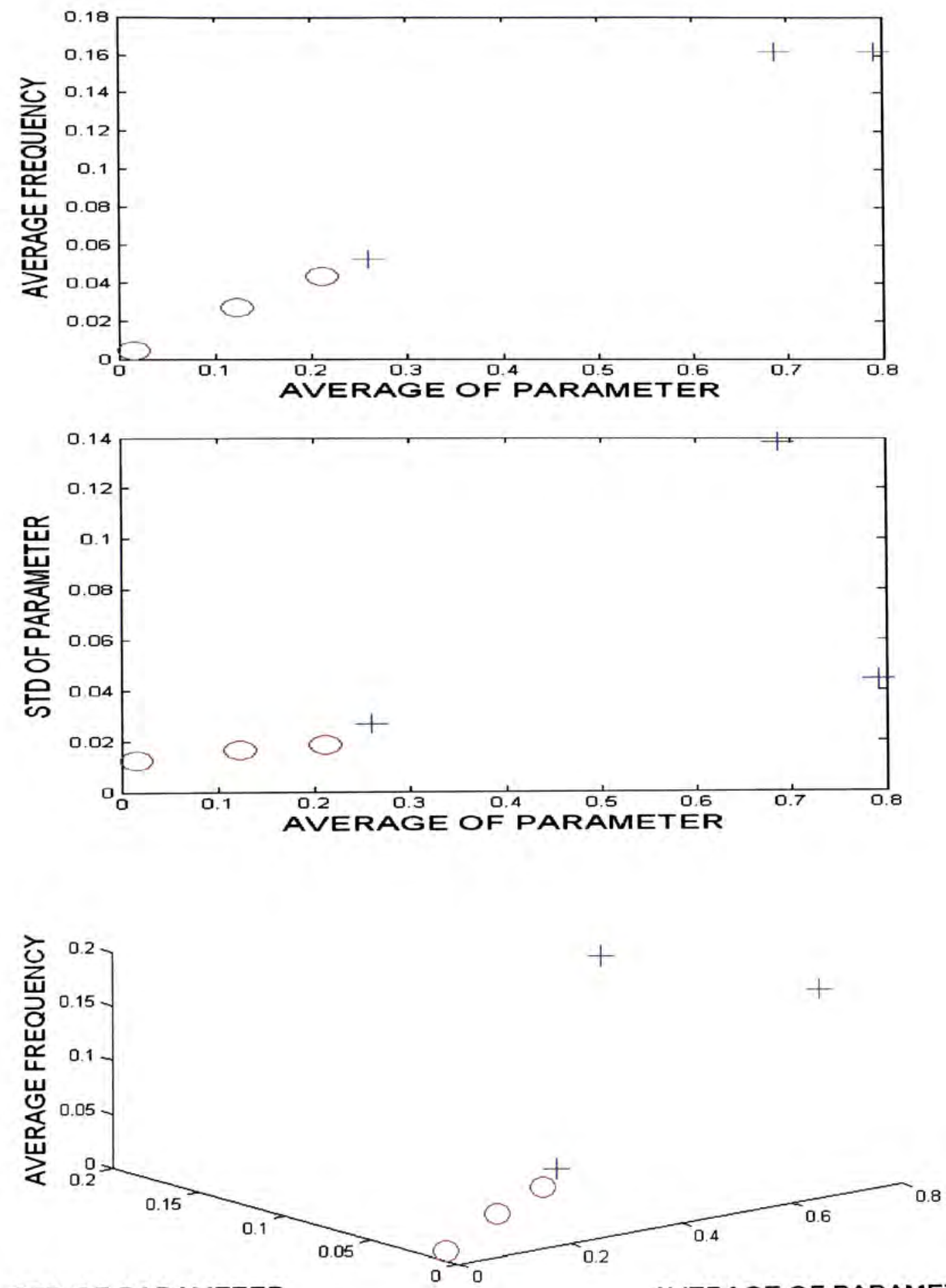

STD OF PARAMETER

AVERAGE OF PARAMETER

(b) Avg. freq. vs. parameter, std vs. avg. of parameter, avg. freq. vs. parameter. Figure 5.21. Complexity results for patient 7 . Note: Leading to seizure (red (o) or red $(-)$ ) and not leading to seizure (blue $(+)$ or blue $(+-)$ ). 

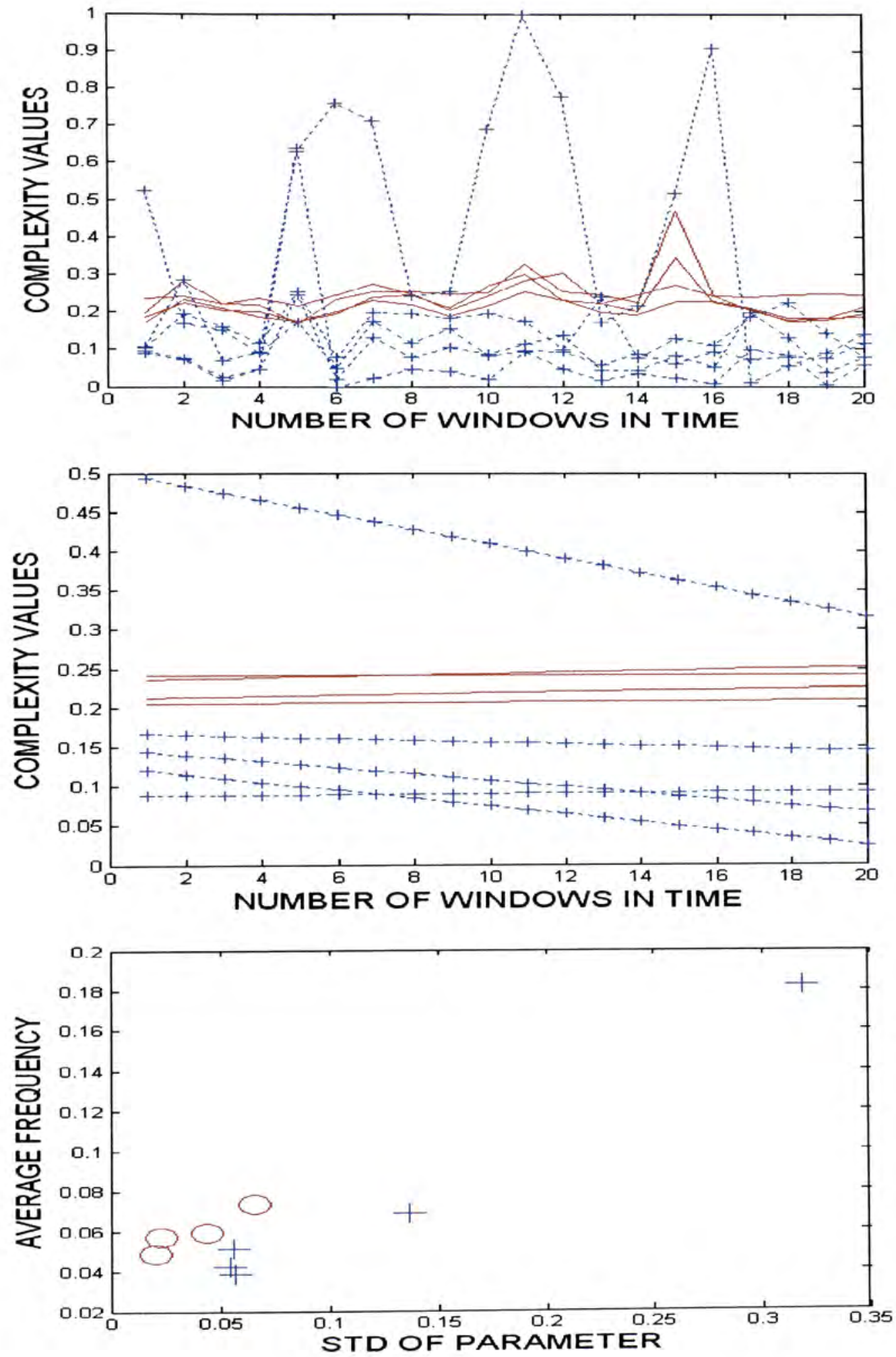

(a) Behavior of parameter, regression lines, average vs. of parameter. 

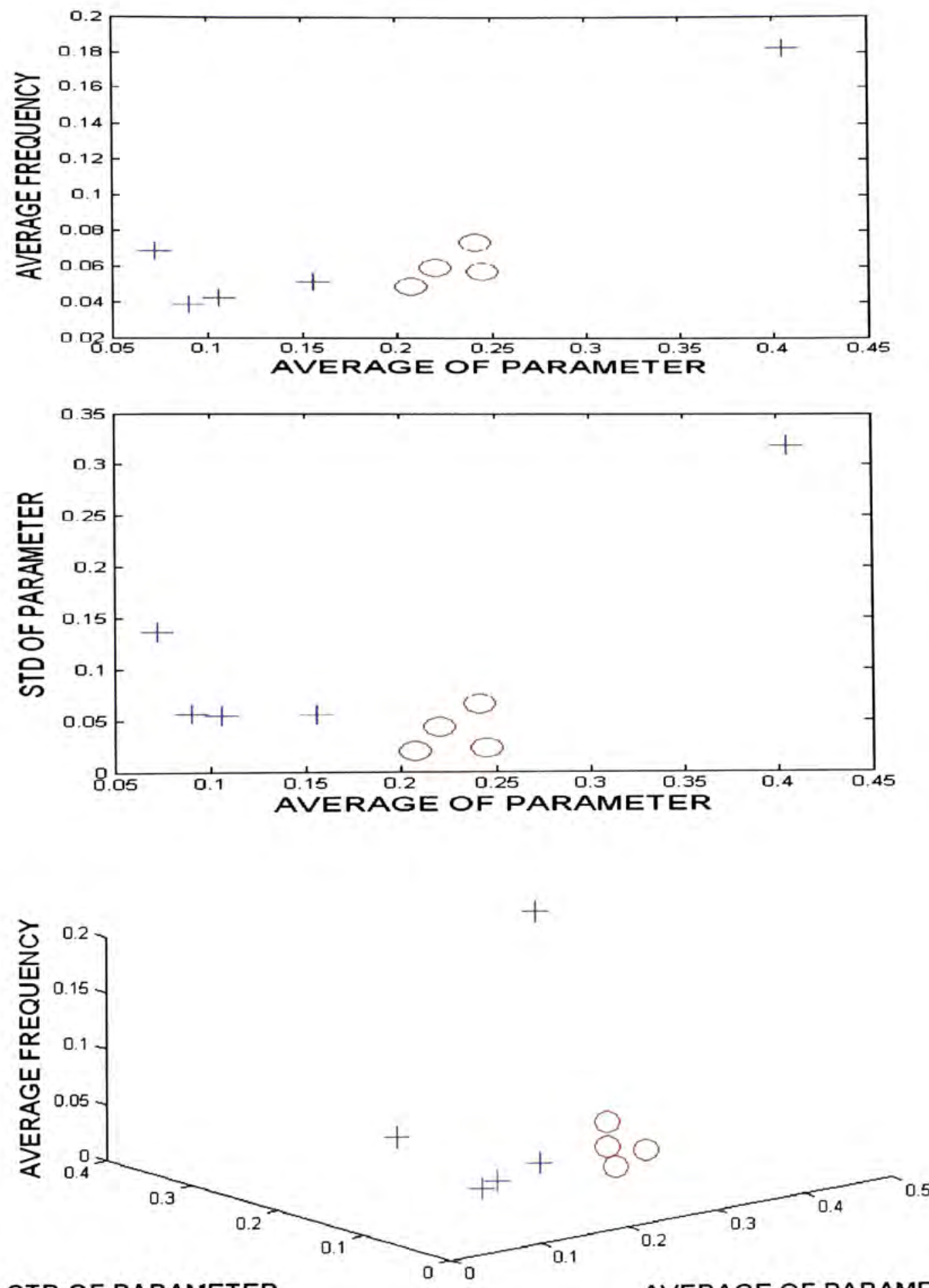

STD OF PARAMETER

AVERAGE OF PARAMETER

(b) Avg. freq. vs. parameter, std vs. avg. of parameter, avg. freq. vs. parameter.

Figure 5.22. Complexity results for patient 8 . Note: Leading to seizure (red (o) or red $(-)$ ) and not leading to seizure (blue $(+)$ or blue $(+-)$ ). 


\subsubsection{DYNAMICS OF CORRELATION INTEGRAL, MOBILITY, AND COMPLEXITY AS POTENTIAL SEIZURE PREDICTORS}

In assessing the examples treated before, the complexity parameter produces the most consistent and reliable results across all 8 patients included in the study. Nevertheless, a detailed example is provided for a long event consisting of one hour of EEG recording prior to a seizure to see how these 3 parameters change as we approach an ictal state. For this particular example, we provide the behavior of the 3 parameters in 2 distinct windows of time for visual appreciation. For each window of time, the behavior of the parameter itself is provided with respect to time as well as through regression line approximations corresponding to that behavior. Their respective 3-D plots with mean, standard deviation, and power of the frequency as $\mathrm{x}, \mathrm{y}, \mathrm{z}$ coordinates are given.

It can be observed, as illustrated in Figure 5.23, in this long event of 1 hour, how the mobility of the EEG signal can differentiate between the two groups of electrodes. Mobility remains the most reliable feature in this case. The regression lines corresponding to this parameter are separated in two well defined groups. Also correlation integral and complexity show similar distinctions as shown in Figures 5.24 and 5.25.

As time approaches the onset of the seizure, the trend of the behavior of the measured parameters through time becomes similar for the two classes of electrodes. In other words, just prior to the seizure, the regression lines of the two groups of electrodes 
converge into one group; there is no clear separation between them as can be seen in Figures 5.26, 5.27 and 5.28.

In Figure 5.26, which shows the mobility plots, it can be observed that 60 seconds prior to the onset of the seizure, the behavior of this parameter becomes the same for all electrodes. The regression line approximations for these signals also show how the two groups (red and blue) converge prior to the onset.

Figure 5.27 shows the complexity parameter over time. Notice between 60 and 80 seconds in the plot, the signals start to converge into one group. Similarly, Figure 5.28 shows the correlation overt time, and it could be seen that 40 seconds before the onset how all electrodes start to behave in a similar fashion.

Figures 5.29, 5.30 and 5.31 illustrate the dynamics of the 3 parameters calculated 8 minutes after the seizure onset, including the seizure itself. Regression lines are provided as well in order to visualize the trend of each parameter.

As illustrated in Figure 5.29, it can be observed from the behavior of the correlation as a parameter, that after the seizure, the regression lines for the two groups of electrodes (red \& blue lines) start to separate from each other, while at the onset they were mixed together. The same reasoning applies for the mobility and complexity parameters as illustrated in Figures 5.30 and 5.31. The plots of the behavior of the correlation, on the other hand, are not as distinct. 

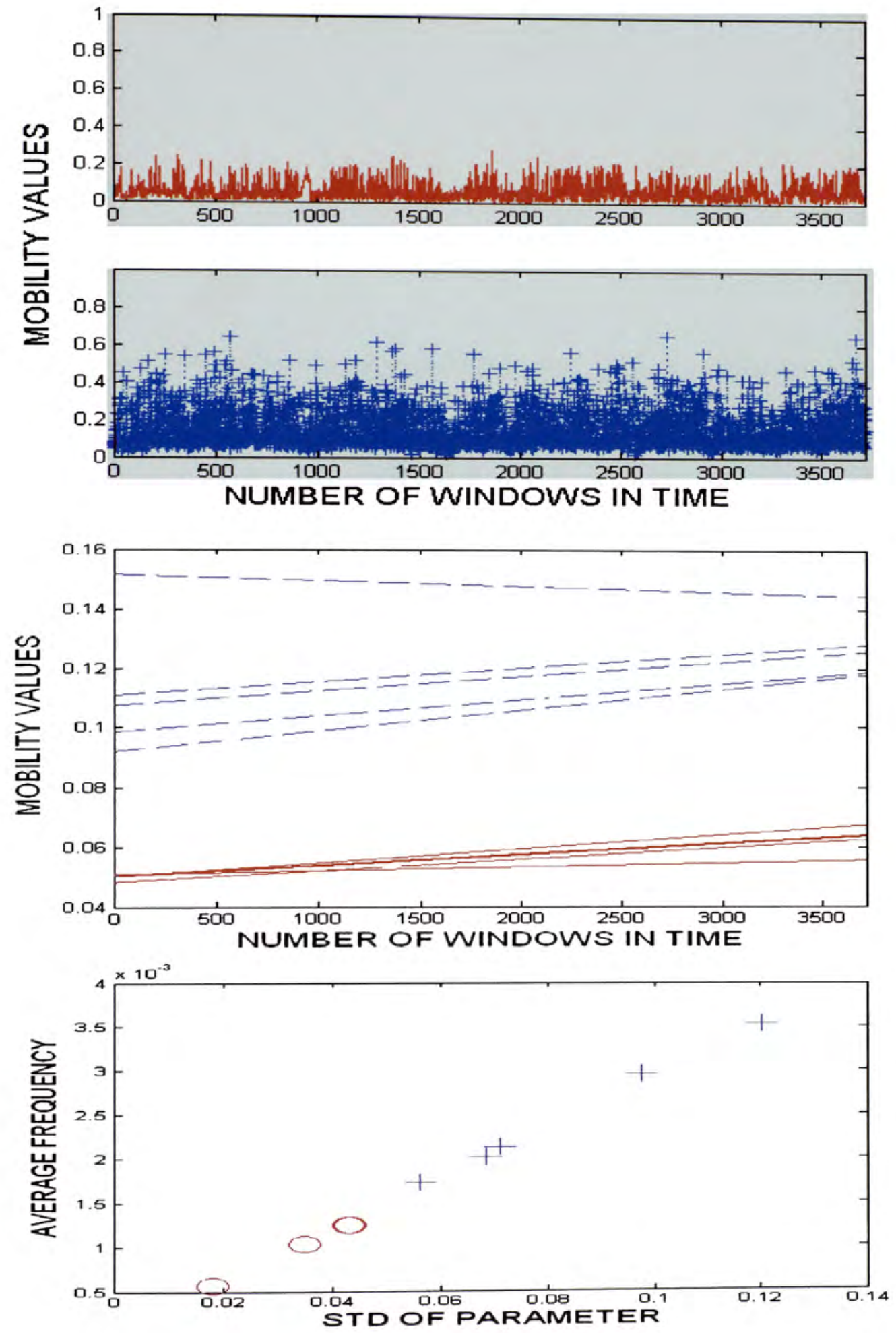

(a) Behavior of parameter, regression lines, average vs. of parameter. 

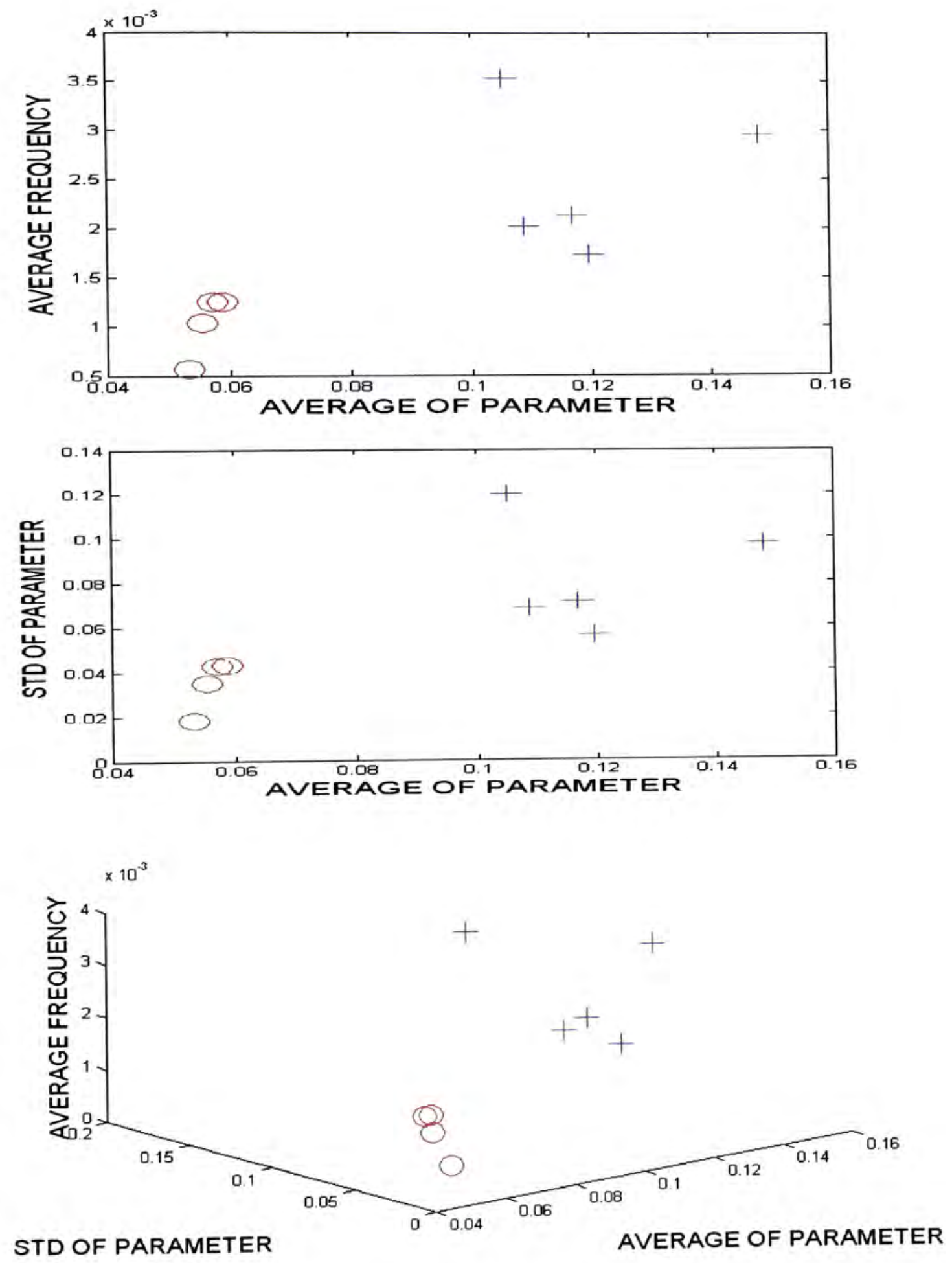

(b) Avg. freq. vs. parameter, std vs. avg. of parameter, avg. freq. vs. parameter. Figure 5.23. Mobility results ( 1 hour before seizure). Note: Leading to seizure (red (o) or red $(-)$ ) and not leading to seizure (blue $(+)$ or blue $(+-)$ ). 

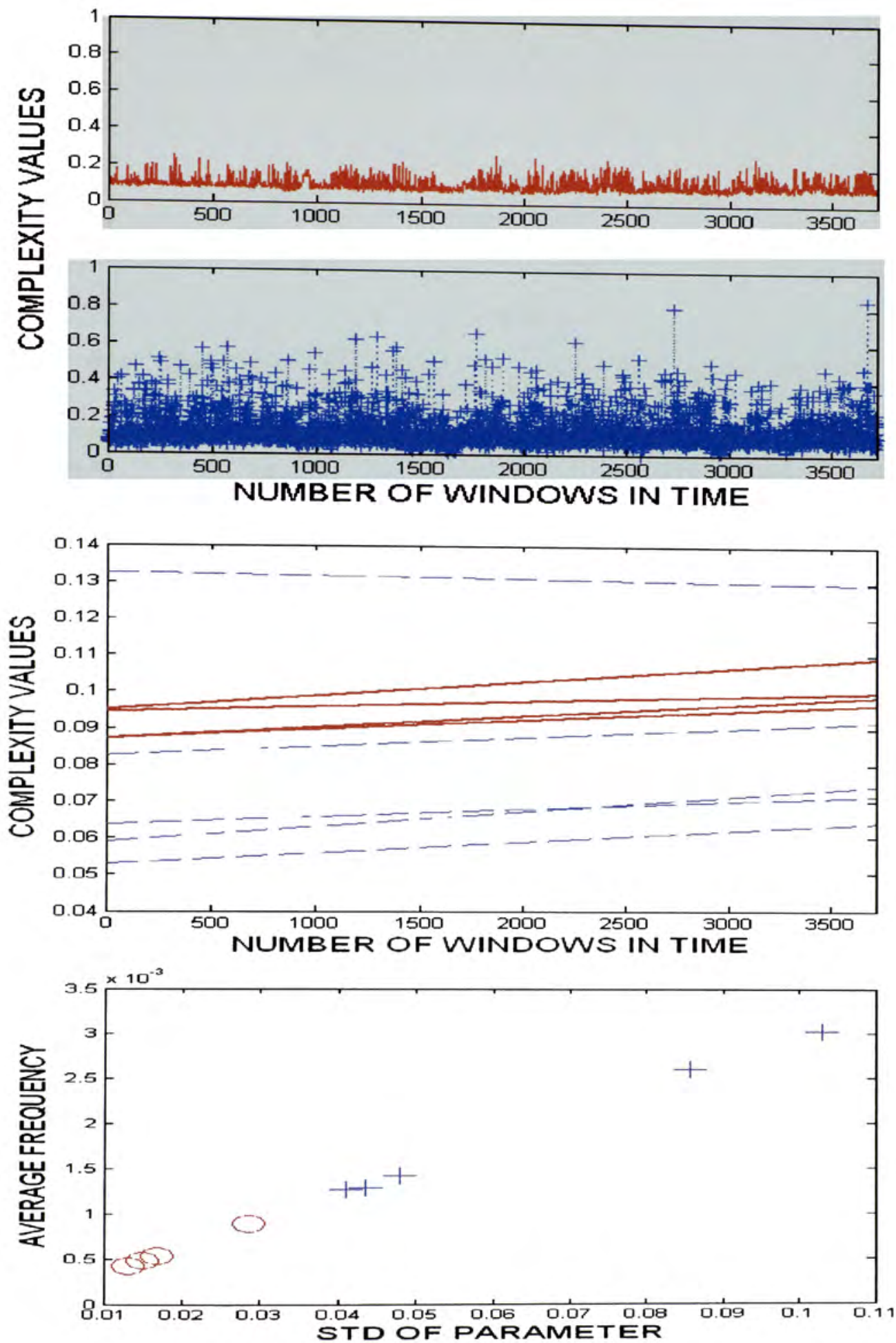

(a) Behavior of parameter (top (leading) \& bottom (not leading)), regression lines, average freq. Vs. STD of parameter. 

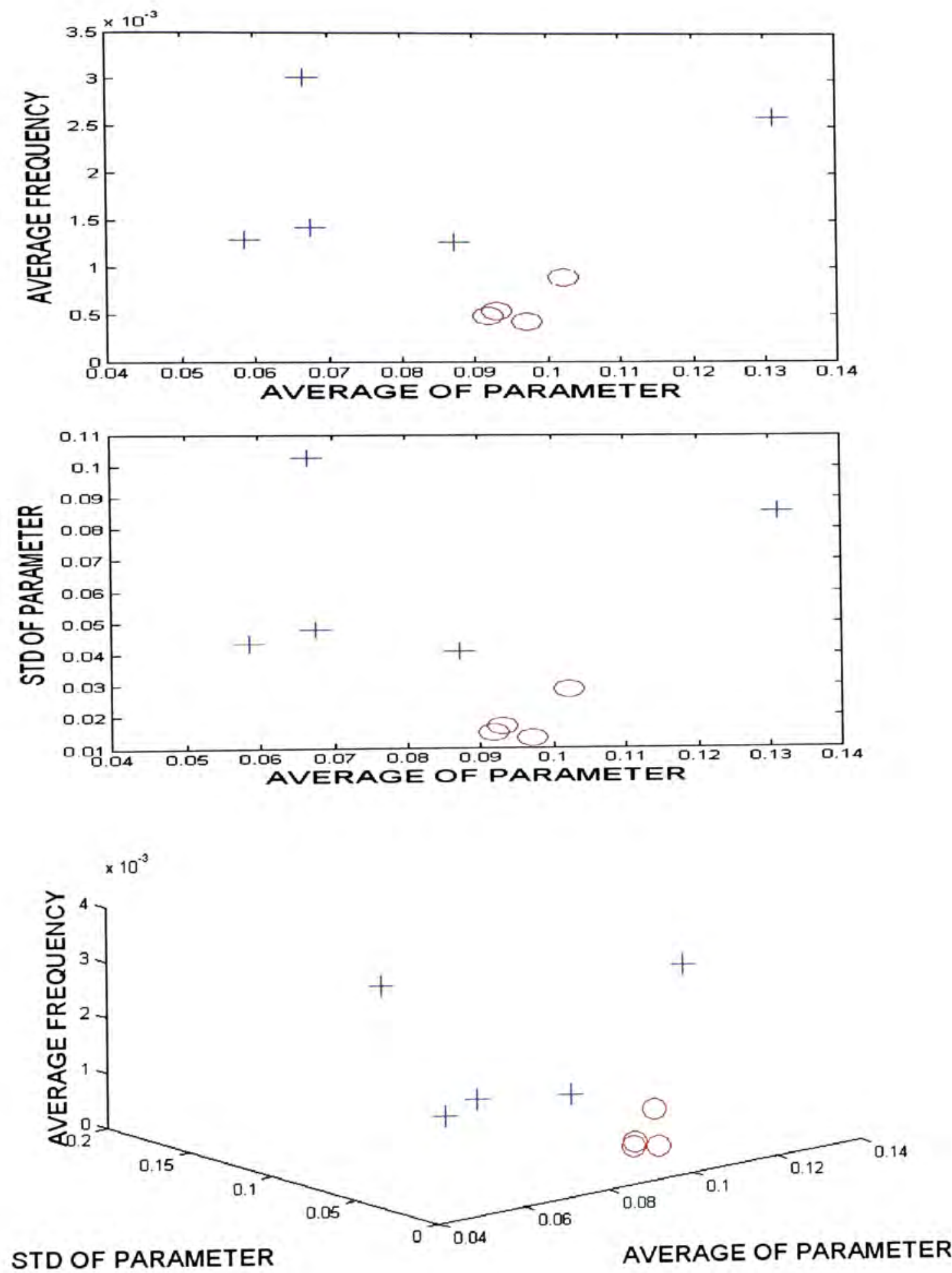

(b) Avg. freq. vs. parameter, std vs. avg. of parameter, avg. freq. vs. parameter.

Figure 5.24. Complexity results (1 hour before seizure). Note: Leading to seizure (red (o) or red $(-)$ ) and not leading to seizure (blue $(+)$ or blue $(+-)$ ). 

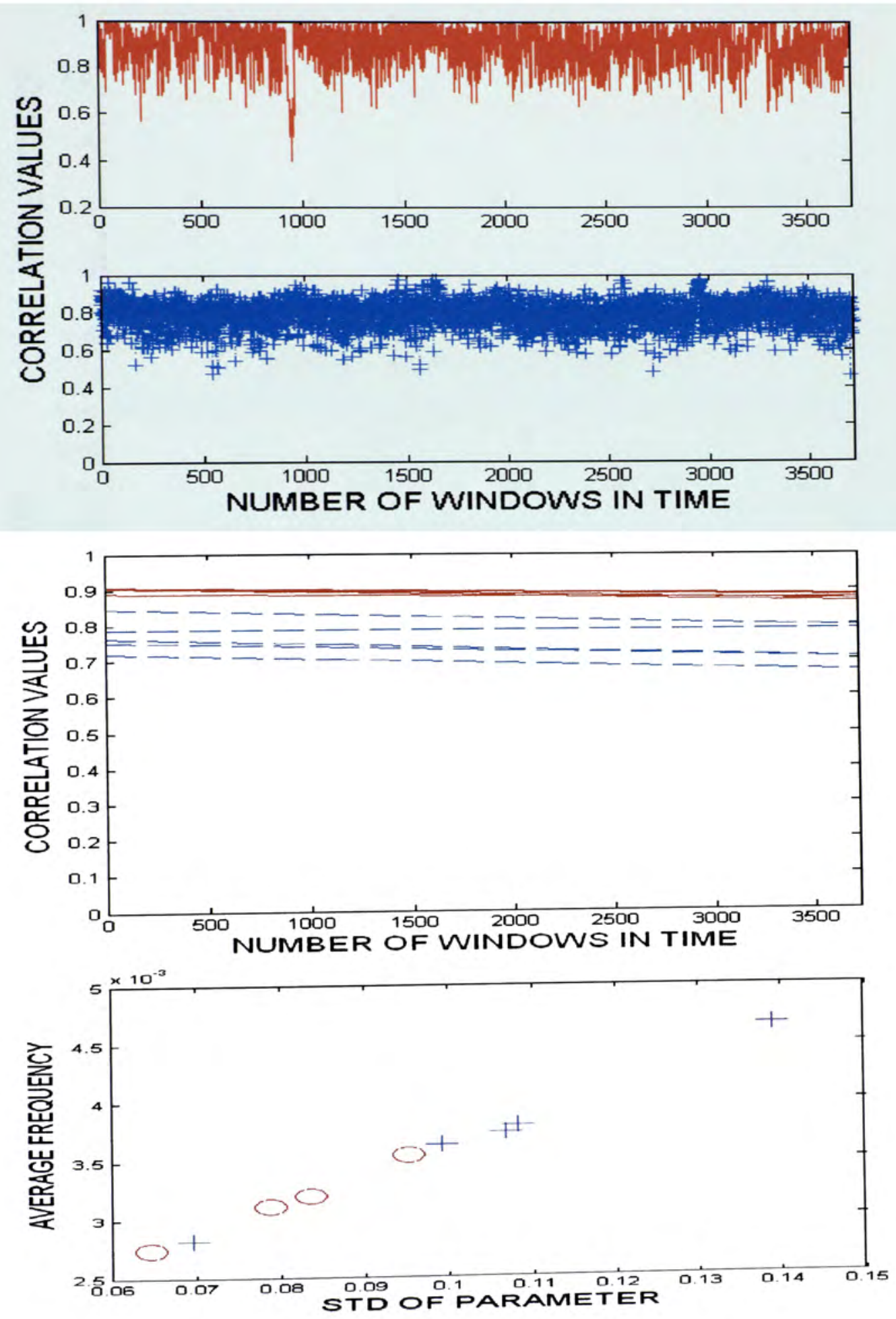

(a) Behavior of parameter (top (leading) \& bottom (not leading)), regression lines, average freq. Vs. STD of parameter. 

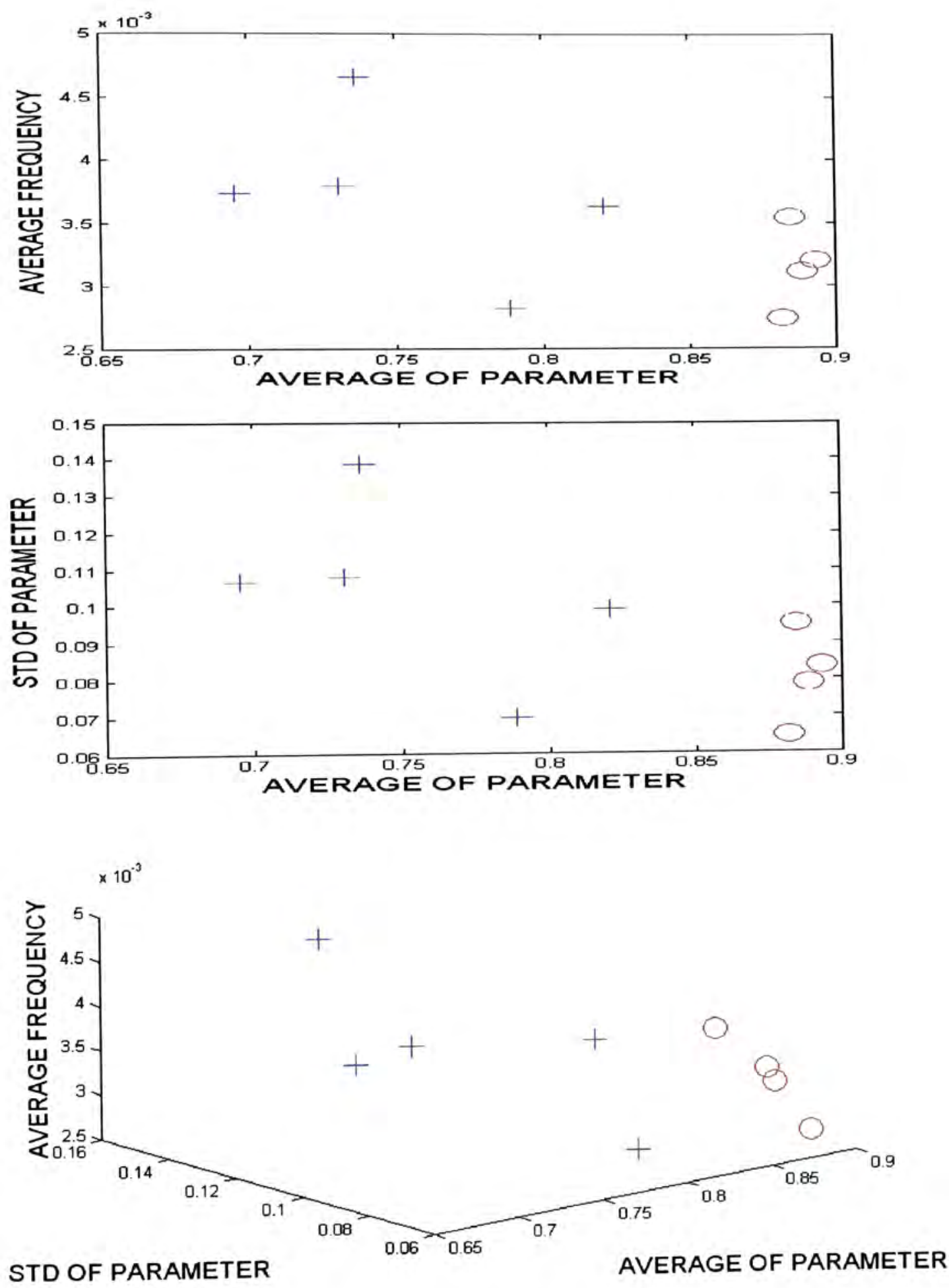

(b) Avg. freq. vs. parameter, std vs. avg. of parameter, avg. freq. vs. parameter. Figure 5.25. Correlation dimension results (1 hour before seizure). Note: Leading to seizure (red (o) or red (-)) and not leading to seizure (blue $(+)$ or blue $(+-))$. 

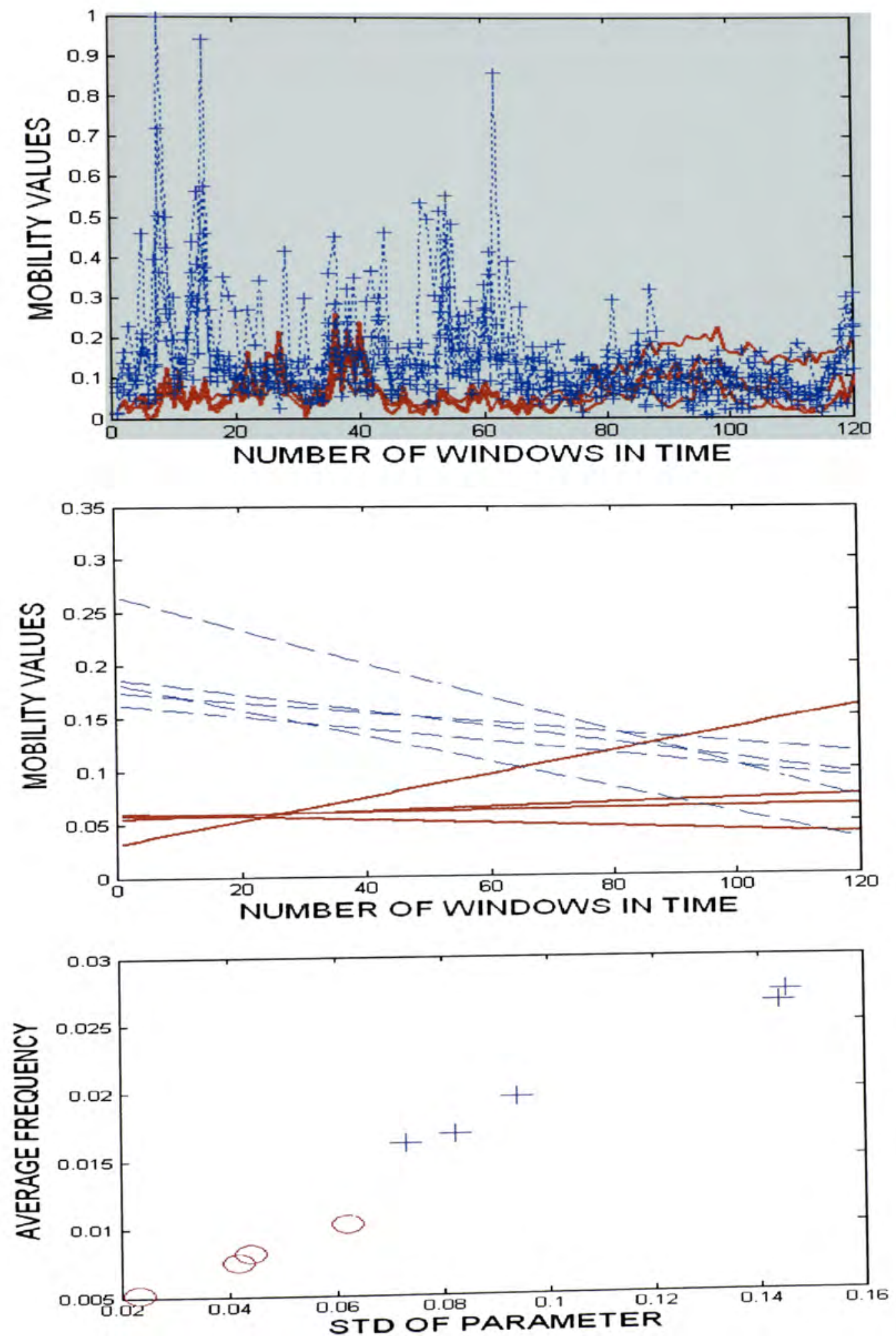

(a) Behavior of parameter, regression lines, average vs. of parameter. 

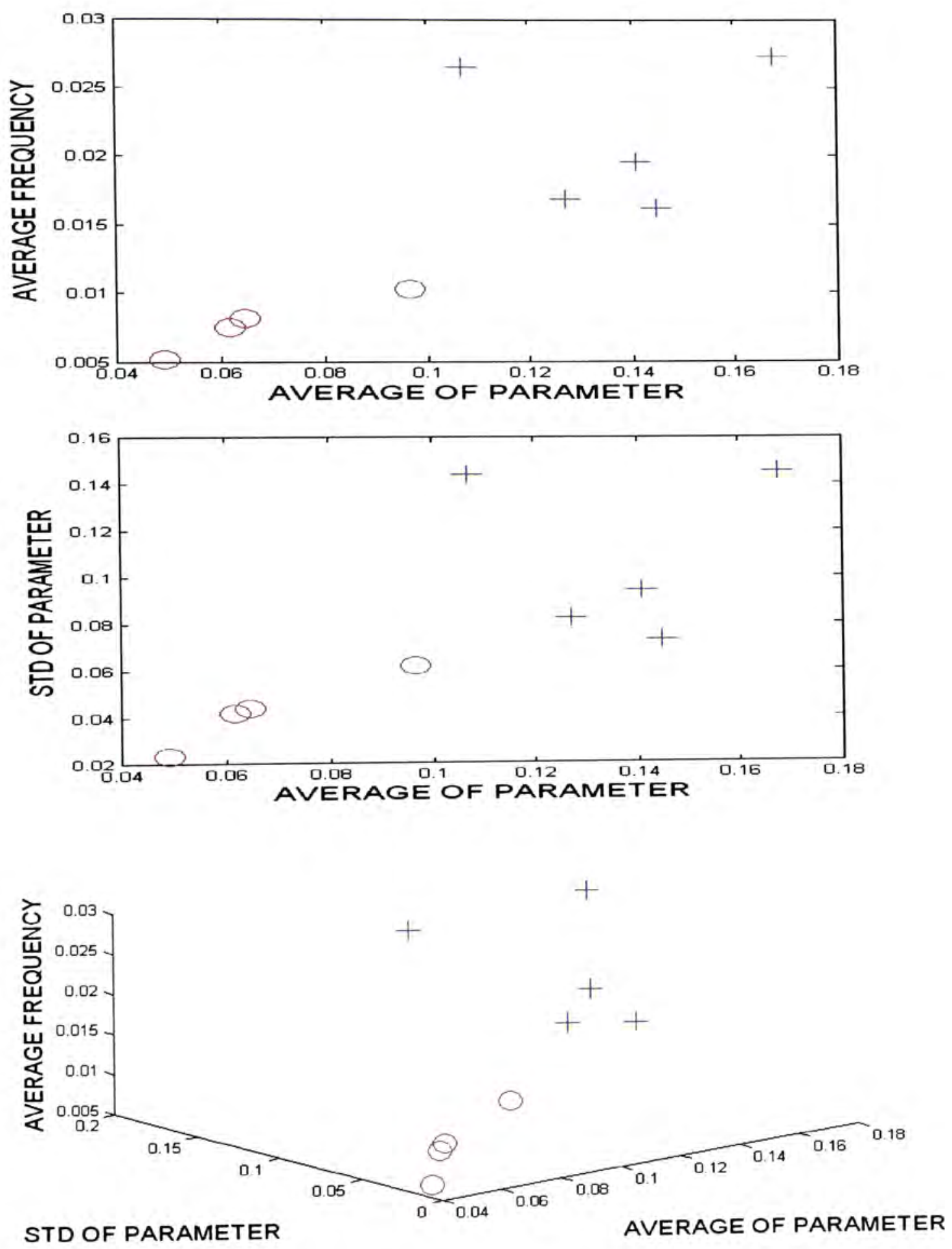

(b) Avg. freq. vs. parameter, std vs. avg. of parameter, avg. freq. vs. parameter. Figure 5.26. Mobility 2 minutes before seizure (seizure onset at 120 seconds). Note: Leading to seizure (red (o) or red (-)) and not leading to seizure (blue $(+,+-,-)$ ). 

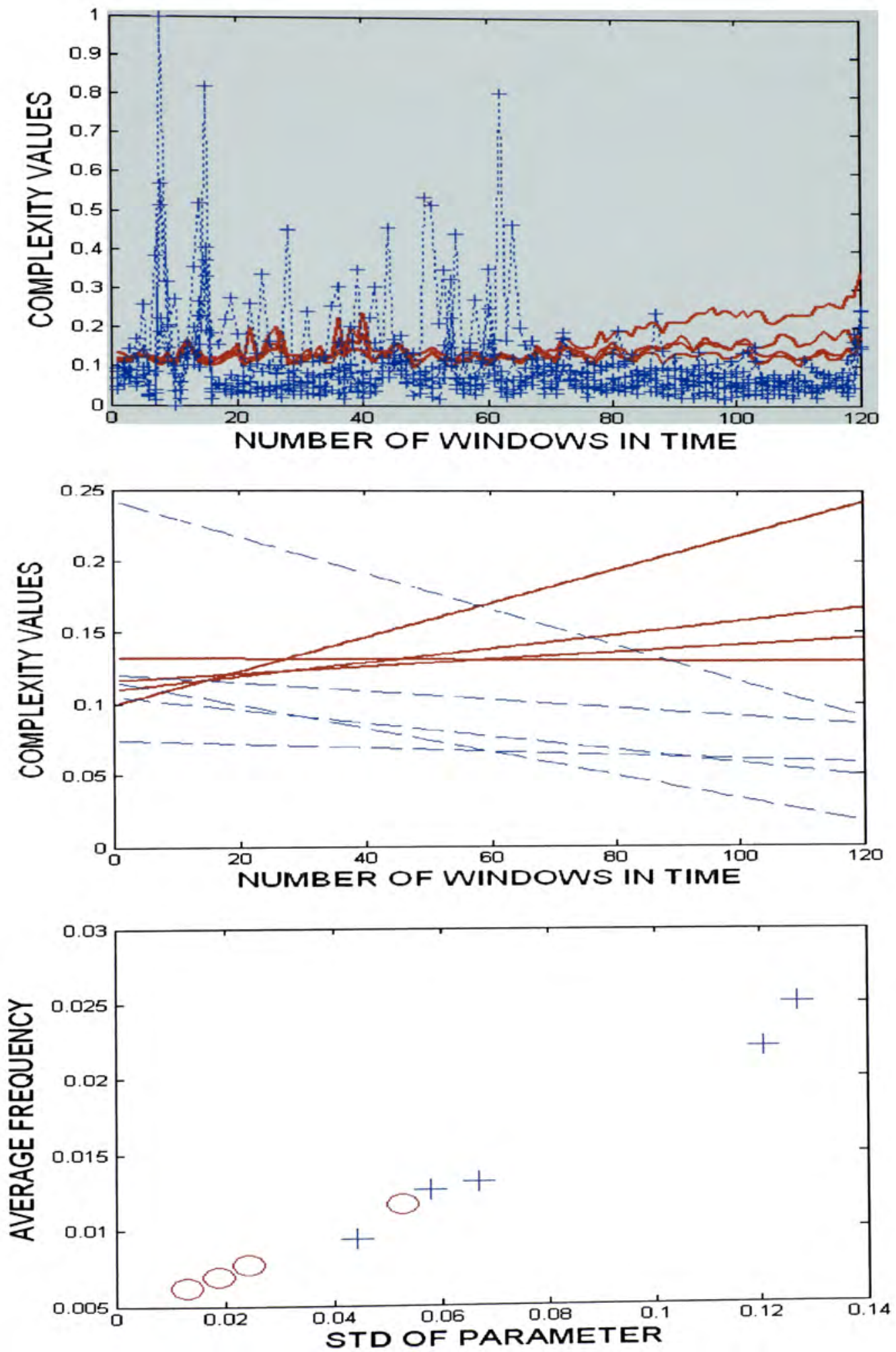

(a) Behavior of parameter, regression lines, average vs. of parameter. 

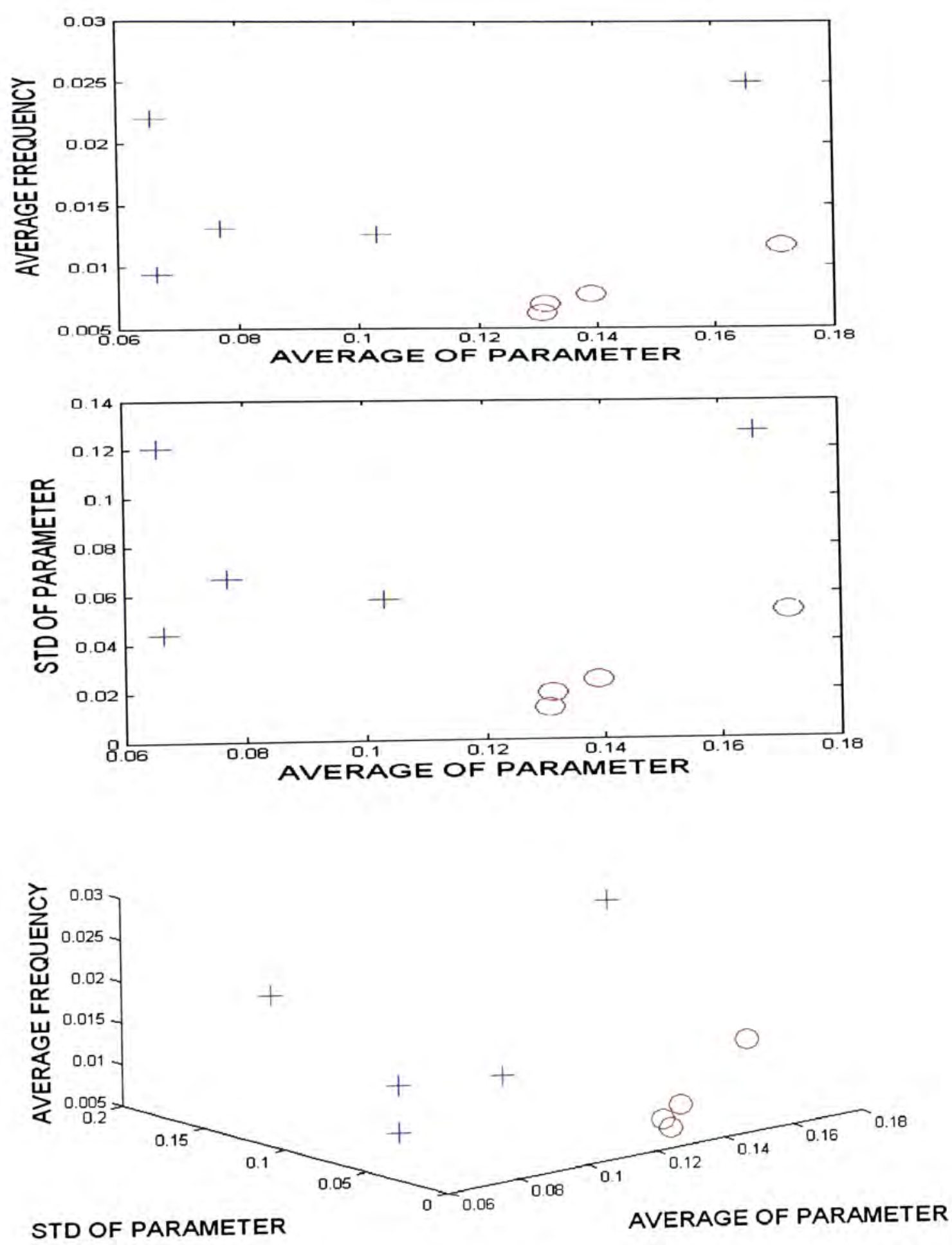

(b) Avg. freq. vs. parameter, std vs. avg. of parameter, avg. freq. vs. parameter.

Figure 5.27. Complexity 2 minutes before seizure (seizure onset at 120 seconds). Note: Leading to seizure (red (o) or red (-)) and not leading to seizure (blue $(+,+-,-)$ ). 

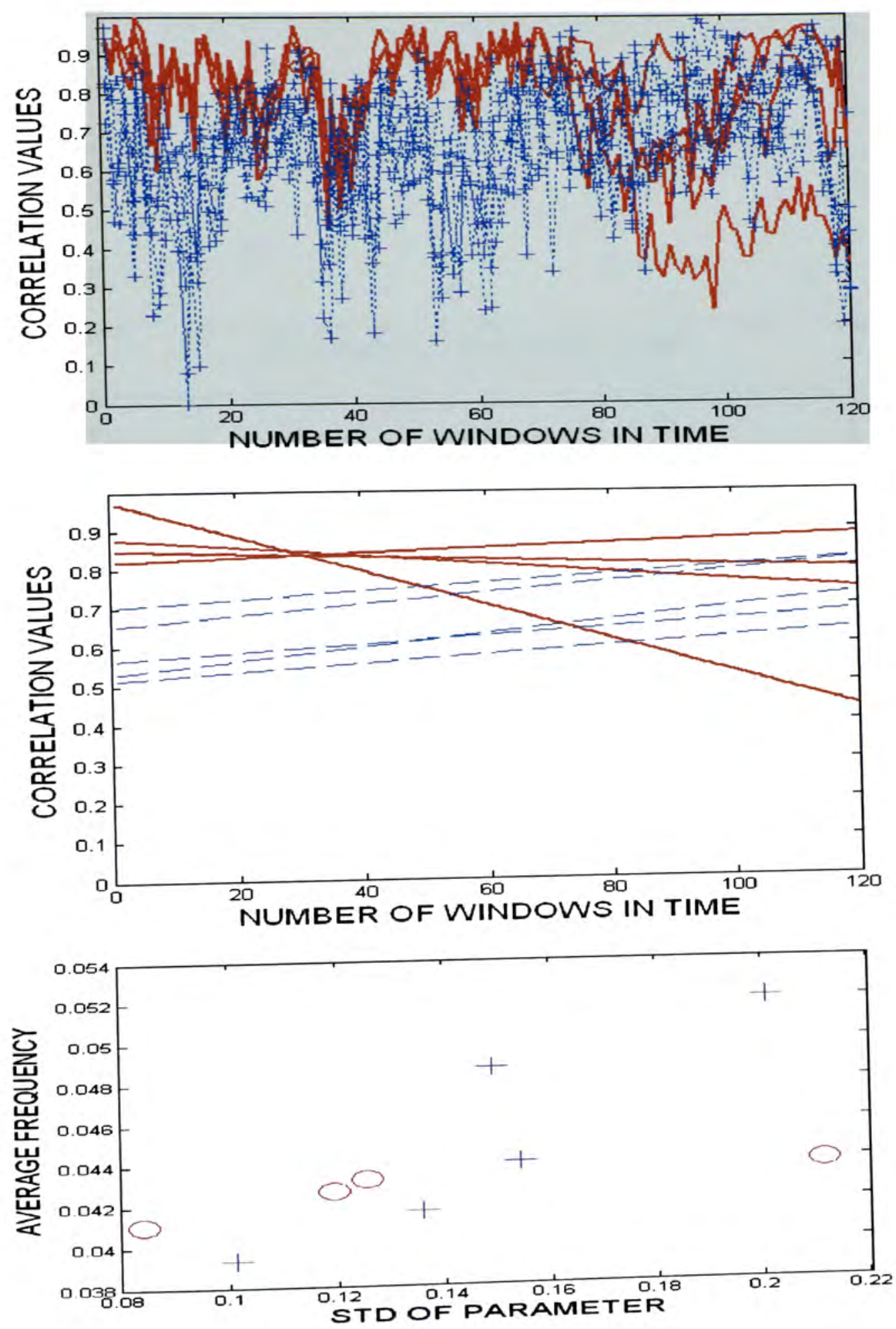

(a) Behavior of parameter, regression lines, average vs. of parameter. 

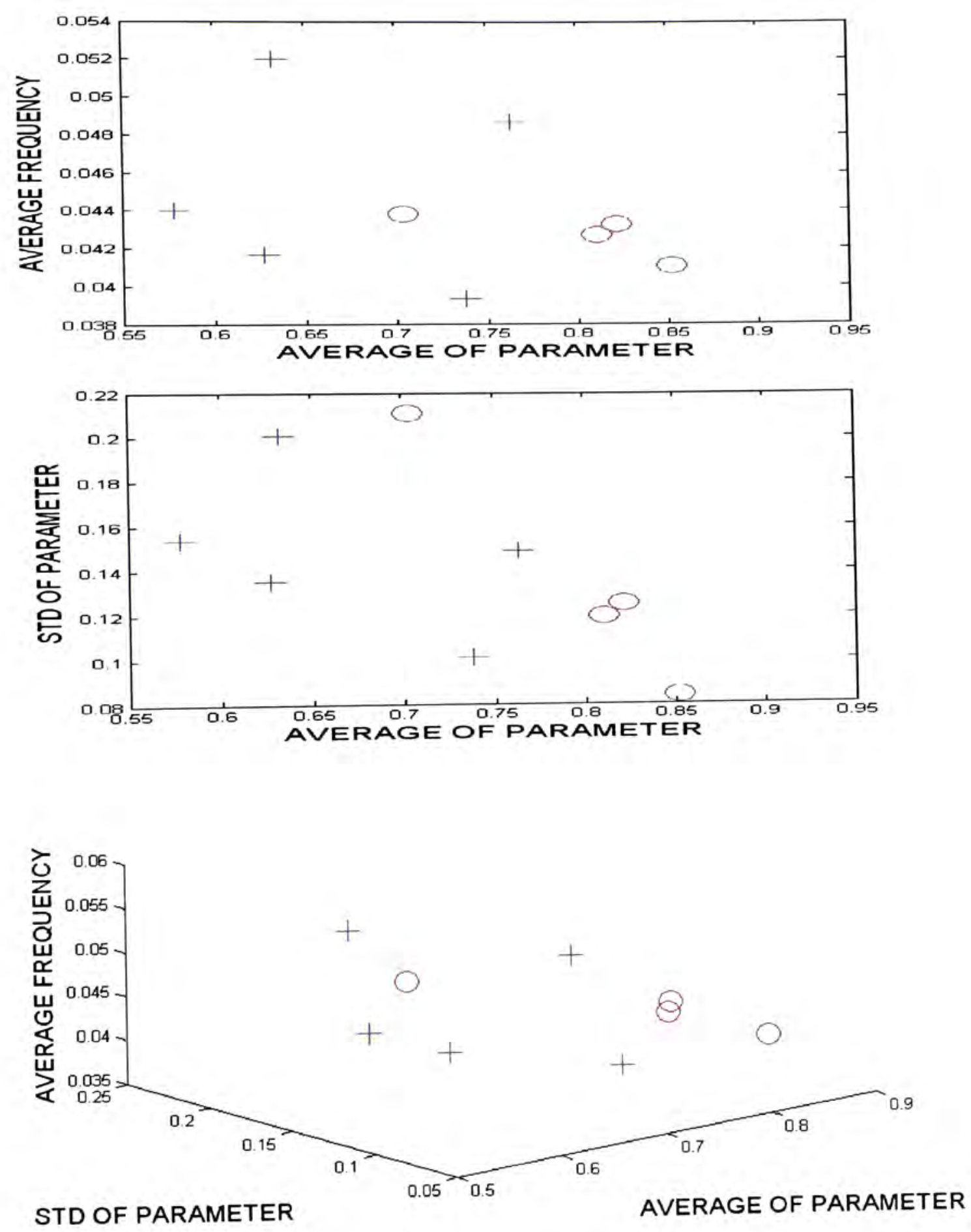

(b) Avg. freq. vs. parameter, std vs. avg. of parameter, avg. freq. vs. parameter. Figure 5.28. Correlation 2 minutes before seizure (seizure onset at 120 seconds). Note: Leading to seizure (red (o) or red (-)) and not leading to seizure (blue $(+,+-,-)$ ). 

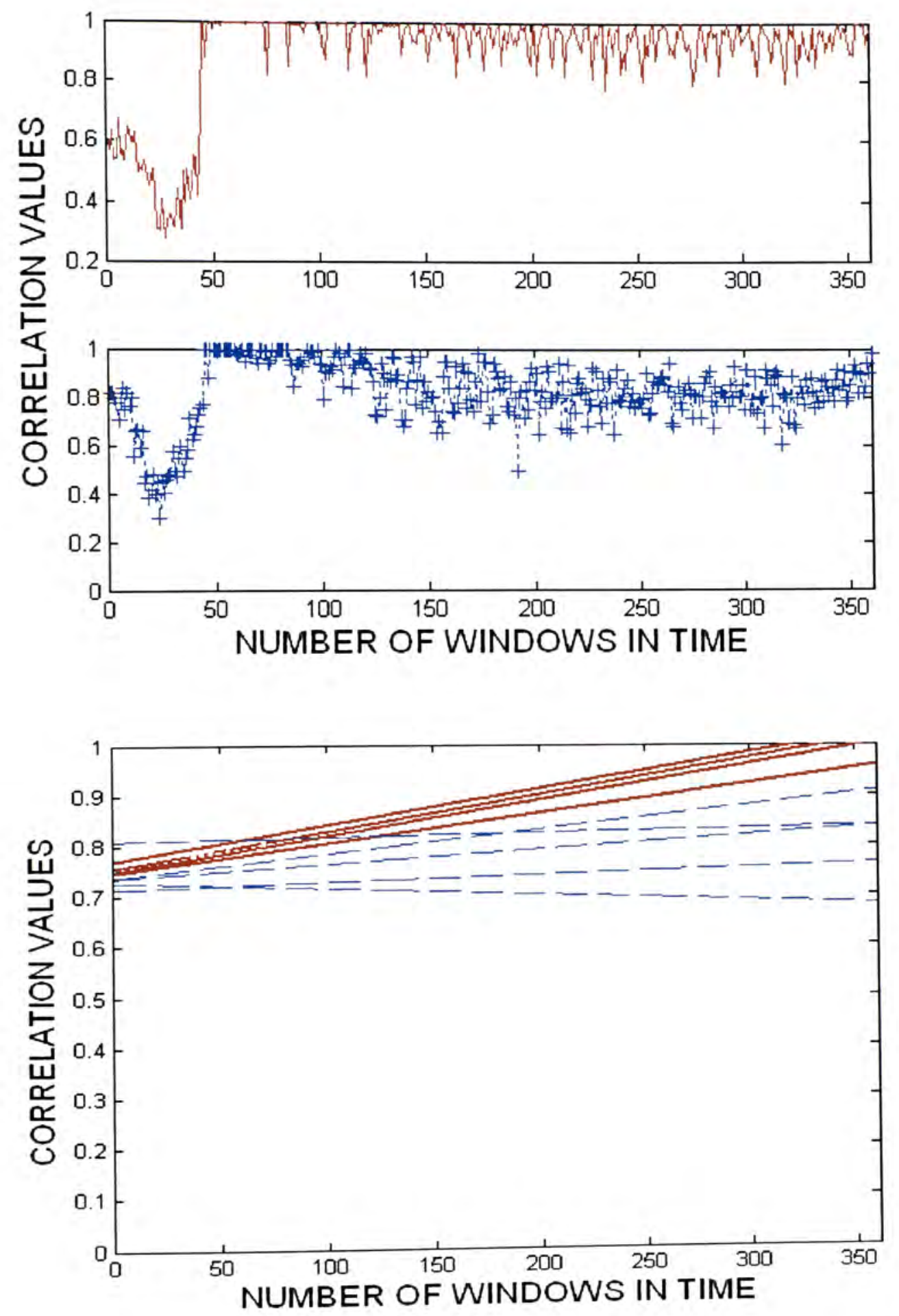

Figure 5.29. Correlation results 6 minutes after seizure including seizure onset. Note: Leading to seizure (red (-)) and not leading to seizure (blue (+-, - -)). 

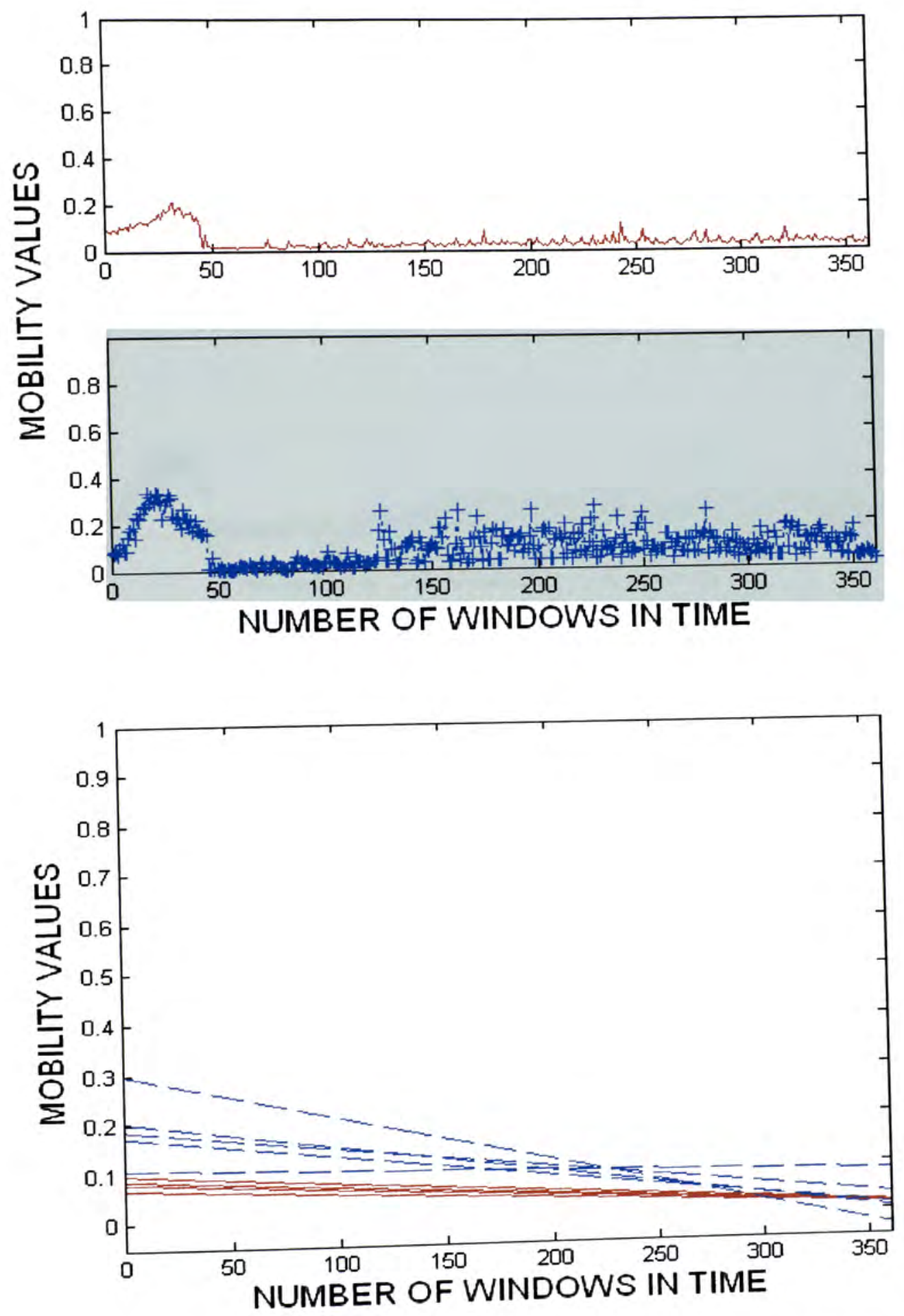

Figure 5.30. Plot of mobility 6 minutes after seizure including seizure onset. Note: Leading to seizure (red (-)) and not leading to seizure (blue (+-, - -)). 

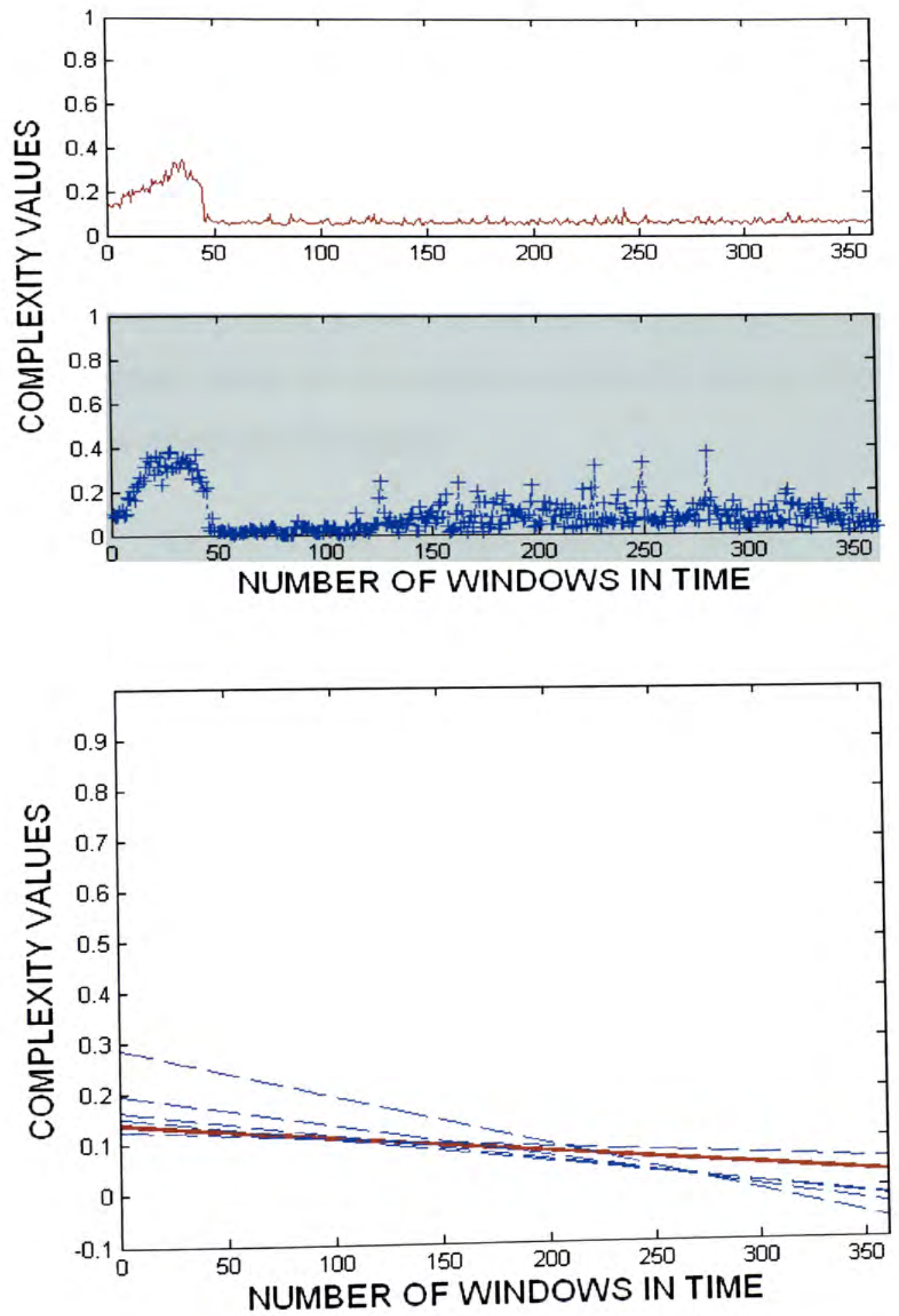

Figure 5.31. Complexity results 6 minutes after seizure including seizure onset. Note: Leading to seizure (red (-)) and not leading to seizure (blue (+-, - -)). 
Table 5.12, provides a summary of the results for of all the patients. The arrows indicate if for a given parameter, the values of the red group of electrodes are higher or lower with respect to the blue group of electrodes (refer to Figure 5.32). As can be observed, for 5 patients out of 8 , the complexity values for those electrodes that lead to an ictal state are higher than the values of those electrodes that do not lead to seizure. Also, the mobility values for these five patients behave in the same manner. Two patients behave in a similar fashion, and their complexity and mobility values are reversed if we compare them with the other five patients.

Table 5.12. Lower $\Downarrow$ or Higher $\Uparrow$ values of the red with respect to the blue channels.

\begin{tabular}{|c|c|c|c|}
\hline Patient & $\begin{array}{c}\text { Mobility } \\
(M)\end{array}$ & $\begin{array}{c}\text { Complexity } \\
\text { (C) }\end{array}$ & $\begin{array}{c}\text { Correlation } \\
(R)\end{array}$ \\
\hline & Red & Red & Red \\
\hline 1 & $\Uparrow$ & $\Uparrow$ & $\Downarrow$ \\
\hline 2 & $\Uparrow$ & $\Uparrow$ & $\Downarrow$ \\
\hline 3 & $\Downarrow$ & $\Downarrow$ & $\Uparrow$ \\
\hline 4 & $\Uparrow$ & $\Uparrow$ & $\Downarrow$ \\
\hline 5 & $\Uparrow$ & $\Uparrow$ & $\Downarrow$ \\
\hline 6 & $\Uparrow$ & $\Uparrow$ & $\Downarrow$ \\
\hline 7 & $\Downarrow$ & $\Downarrow$ & $\Uparrow$ \\
\hline 8 & $\Downarrow$ & $\Uparrow$ & $\Uparrow$ \\
\hline
\end{tabular}

A closer look at this table reveals the following conditions: If we assign a negative $(-)$ to $\Downarrow$ and a $(+)$ to $\Uparrow$, then, the following relations hold. These relations as established in equation 5.16 constitute another mayor finding in this dissertation.

$$
\begin{aligned}
& \mathrm{C} * \mathrm{R}=- \\
& \mathrm{M} * \mathrm{R}=- \\
& \mathrm{M} * \mathrm{C}=+ \\
& \mathrm{M} * \mathrm{C} * \mathrm{R}=-
\end{aligned}
$$



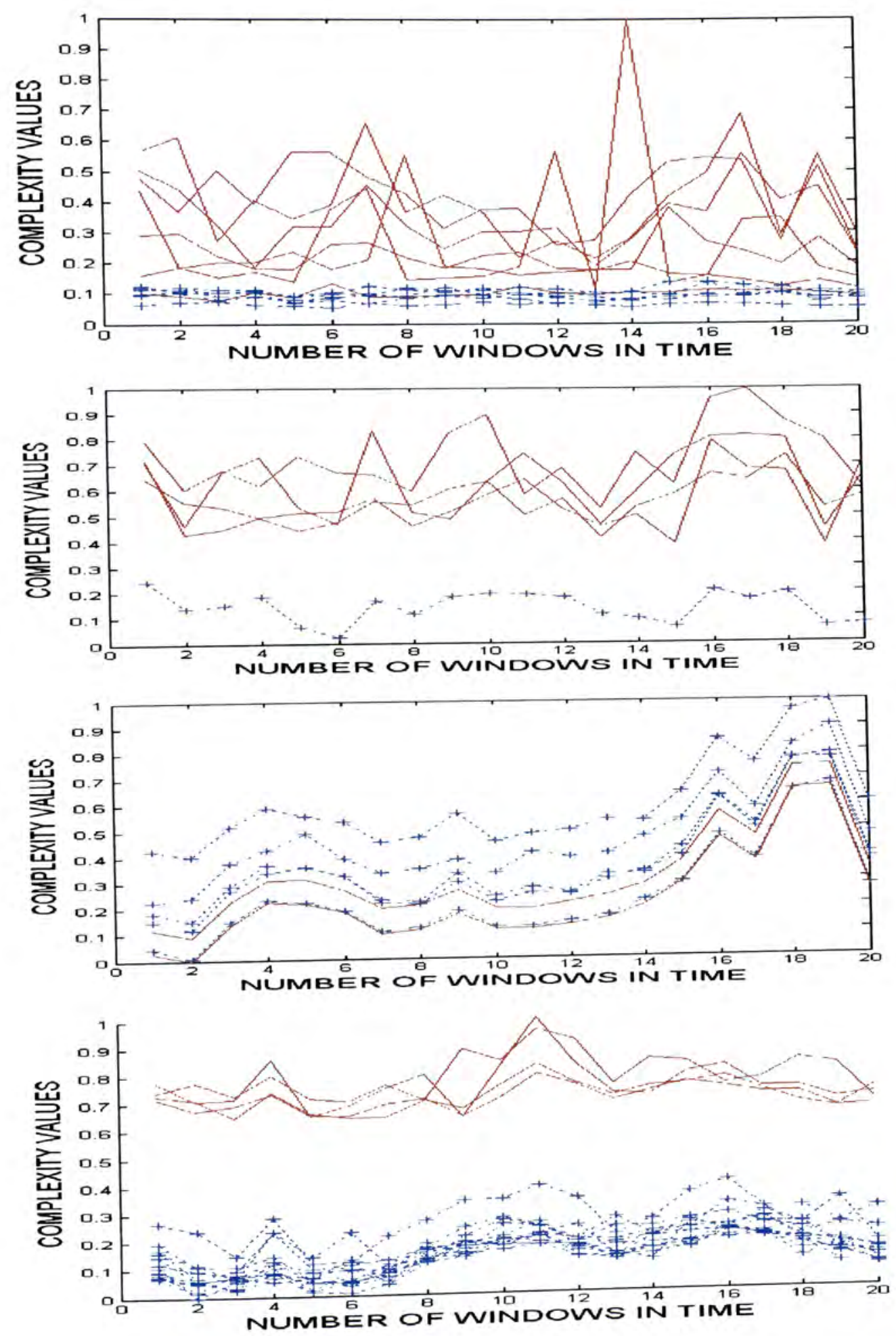

(a) Complexity results for patients 1-4. 

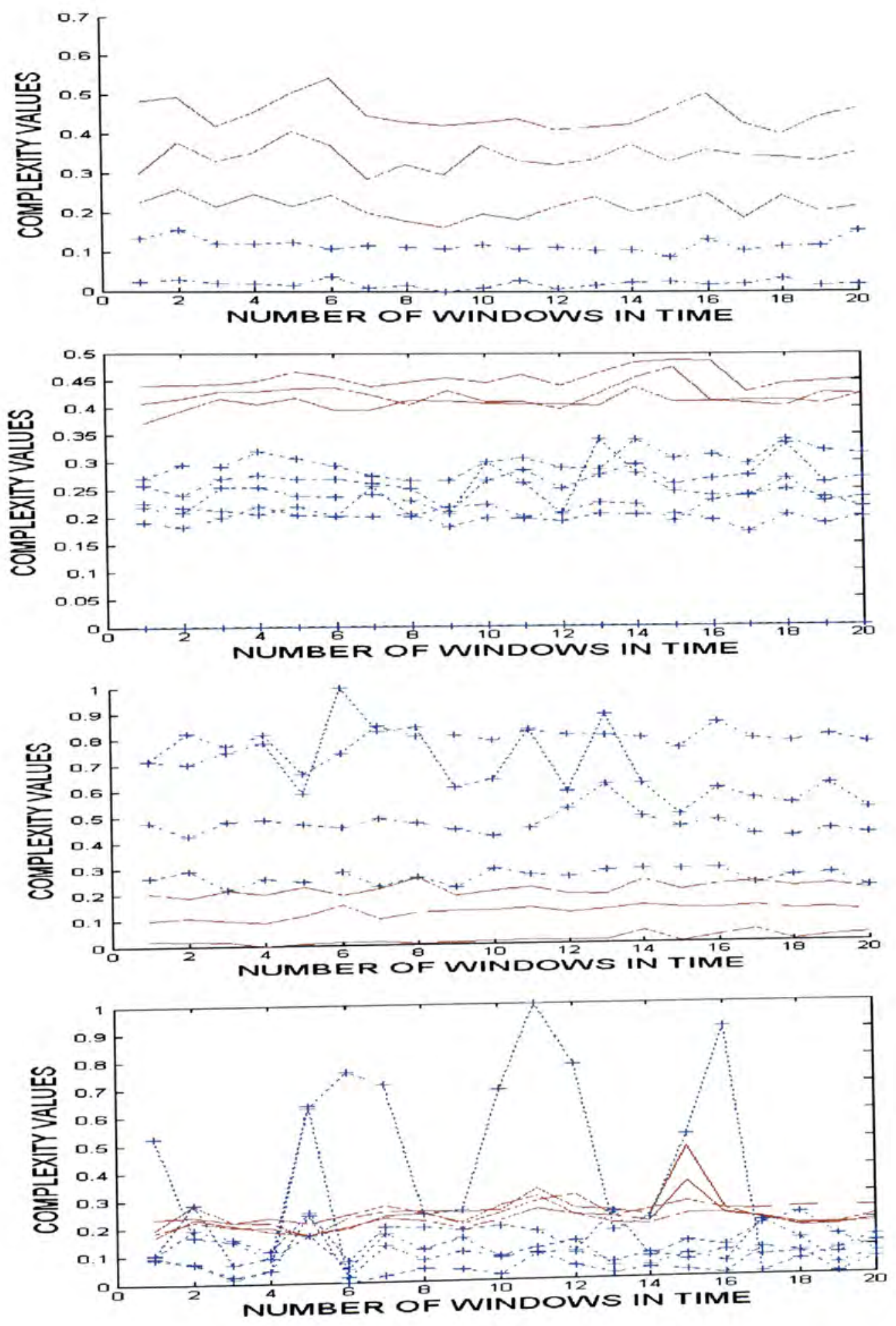

(b) Complexity results for patients 5-8.

Figure 5.32. Complexity results for all patients. Note: Leading to seizure (red (-)) and not leading to seizure (blue (+-)). 


\section{CHAPTER 6}

\section{CONCLUSION}

This dissertation serves as groundwork on the study of the subdural interictal EEG data. In this study, characterizing patterns of interictal EEG data were formulated and evaluated using different features in order to extract similar behaviors in the electrodes that lead to an ictal state in contrast to those that do not. We translated each of the observable characteristics into mathematical expressions such that each and every one of the characteristics is implemented in the development of the algorithm.

Chapter 2 provided a description of the system used for recordings and the practical design aspects involved in carrying out the experiments. A background of the ictal and interictal states of the EEG was explained in details. Issues pertaining to the clinical relevance of the study towards enhanced EEG analysis, dilemmas imposed by the ubiquitous, recording particularities, and montage were all addressed in order to facilitate replication at other sites that share similar research goals. The infrastructure made available through the Neuroscience Center at Miami Children's Hospital facilitated this research work and helped not only in the implementation part, but also in carrying out the feasibility studies needed to confirm the soundness of the algorithm.

Chapter 3 is a precursor study to the classification algorithm that is detailed in Chapter 5, which assumes all electrodes to have interictal spike activity. This chapter introduces a new method for automatically detecting interictal spikes present in the EEG data recorded. 
Chapter 4 addressed preliminary EEG data manipulation with the main purpose of findings key parameters to analyze the interictal EEG signals. In this Chapter, the focus was placed on deriving mathematical formulas in order to optimize the classification process. The main goal was to acquire an understanding of the behavior of all these electrodes that present particular patterns that will help augment the classification process.

Chapter 5 explained the complete procedure followed in order to interpret the interictal EEG signals before, and after a seizure onset. Through the development of an automated process, a classification algorithm was performed in order to delineate those electrodes that lead to seizure from those that do not. 3D representations of the features obtained from the signals are also provided for enhanced visualization the complex brain patterns. The results obtained present distinguishable behaviors between the two types of electrodes.

The objectives attained included:

- Extracting features that best characterize those EEG electrodes that lead to the onset of a seizure.

- Establishing mathematical derivations that provide not only quantitative measures, but also describes and locates the focus of an ictal activity.

- Correlating the clinical features with the EEG findings in order to determine whether the patient has a consistent source of ictal activity, which is coming from the location concerning the group of electrodes that present interictal activity. 
- Classifying and to group those EEG channels that are known in advance to lead to the onset of a seizure in order to extract similarities in their behavior, so a common behavioral pattern could be found.

- Implementing regression lines as linear approximations of brain dynamics and observe visually among all the discriminating parameters used, which ones led to optimized results across all patients.

- Automating through neural networks the classification process by determining in a new constructed feature space, which was used as input space to the ANN decision functions, in order to separate electrodes that lead to seizure from those that do not, prior and after a seizure onset?

All of these objectives were addressed through the analysis of key parameters applied to the EEG signals.

The results obtained reveal the following findings:

(1) It could be concluded that for the two features of mobility and complexity, there was an evident trend in their outcome since both were higher for those electrodes leading to seizure. This indicates that more sharp fluctuations are present in the EEG amplitude.

(2) There were substantial but characterizing differences in the computed correlation parameter. This calculation varies among different subjects and different types of subdural interictal EEG data. 
(3) The integration of several parameters (correlation integral, mobility, and complexity) constitutes a unified method for assessing differences in the EEG channels that can lead us to classify in a suitable manner the two groups of electrodes.

(4) It will be futile to search for decision functions that separate the two groups of electrodes across patients because experiments reveal that such decision functions are patient dependent;

(5) It is important to know that when one is to search for such decision functions, all electrodes containing interictal activity should be analyzed only if they are localized in different locations inside the brain and with recorded interictal spikes firing at different times.

(6) The selection of key discriminating parameters (correlation integral, mobility, and complexity) used in the implementation.

(7) The unique features that were used to optimize the delineation process of these two types of electrodes, and to get such decision functions.

(8) The development of a back-propagated neural network that automated the decision making process for every patient independent of the others.

(9) The establishment of mathematical functions that elicited the behavior of the key parameters. 
Given the complex nature of EEG recordings, these results, which are supported by clinical experimentation, are most encouraging considering that the patterns applied were computed using data segments of the interictal EEG data irrespective of time and relative position to the onset of a seizure. The integrated algorithm proposed is computationally efficient, fully automated, and designed in a unique way for the extraction of discriminating EEG features. These findings could help in the future to serve as potential predictors of seizure. The use of ictal EEG will likely enhance the precision of the results and add to the merit of this technique. An extension to this research endeavor is one that would integrate internal and external EEG recordings. The results of this integrated technique could be our next research step in order to minimize the number of surgical interventions.

The main contribution of this dissertation is eliciting a new understanding of the characteristics of different interictal epileptiform activities in direct relation to seizure onset. In all performance values of the 3 features implemented, it can be said that the results obtained show great promise in delineating electrodes that lead to seizure from those that do not. It is fitting to note that when the results failed to discriminate between these two classes of electrodes, a clinical analysis revealed that those electrodes were indeed situated in the same region and their interictal spikes were happening simultaneously. As this study will involve a higher number of patients as they become available, additional results will provide more credence to these findings.

The uniqueness of this algorithm is in the establishment of a mathematical foundation capable of extracting features from interictal EEG signals using key 
discriminating parameters, which served as change indicators for our analysis. The relevance is in determining among all of these channels, all containing interictal spikes that occur asynchronously, and located in different regions, which channels are leading and not leading to seizure. 


\section{REFERENCES}

Adjouadi M., Cabrerizo M., Ayala M., and Mirkovic N. "Seizing lesions in 3-D", IEEE Potentials, December/January, 2006.

Adjouadi M., and Candocia F., "A stereo matching paradigm based on the Walsh transformation," IEEE Trans. on Pattern Analysis and Machine Intelligence, 1994, vol. 16(12), pp. $1212-1218$.

Adjouadi, M., Cabrerizo, M., Ayala, M., Sanchez, D., Jayakar, P., Yaylali, I., and Barreto, A., "A New Approach to the Analysis of Epileptogenic Data Using Statistically Independent Operators”, J. Clin. Neurophysiol., Vol. 22(1), pp. 53-64, Jan/Feb 2005.

Adjouadi M., Sanchez D., Cabrerizo M., Ayala M., Jayakar P., Yaylali I., and Barreto A. Interictal Spike Detection Using the Walsh Transform. IEEE Transactions on Biomedical Engineering, Vol. 51 (5), pp. 868-873, May 2004.

Adjouadi, M., Sanchez, D., Cabrerizo, M., Ayala, M., Jayakar, P., Yaylali, I., and Barreto, A., "Interictal Spike Detection Using the Walsh Transform", IEEE Transactions on Biomedical Engineering, Vol. 51, No. 5, pp.868-873, May 2004.

Ayala M., Adjouadi, M., Yaylali, I., and Jayakar, P. "An Optimization Approach to Recognition of Epileptogenic Data Using Neural Networks with Simplified Input Layers", Biomed. Sciences Instrumentation, Vol. 40, pp. 181-186, 2004.

Barkley G.L., and Baumgartner C., MEG and EEG in Epilepsy. J. Clin Neurophysiol, 2003, vol. 20 (3), pp. 163-178.

Barreto A.B., Principe, J.C. and Reid, S.A, "STL: A spatio-temporal characterization of focal interictal events", Brain Topography, 1993, vol. 5(3), pp. 215-228.

Barreto A. B., Principe J. C. and Reid S. A., "STL: A Spatio-Temporal Characterization of Focal Interictal Events”, Brain Topography, Vol. 5, No. 3, pp. 215-228, Human Sciences Press, New York, 1993.

Birkemeier W. P., Fontaine A. B., Celesia G. G., Ma K. M., "Pattern Recognition Techniques for the Detection of Epileptic Transients in EEG," IEEE Transactions on Biomedical Engineering, BME-25 (3), 1978, pp. 213 - 217.

Blume W.T., Holloway G.M, and Wiebe S., Temporal epileptogenesis: localizing value of scalp and subdural interictal and ictal EEG data. Epilepsia, 2001 Apr; 42(4), pp.508514.

Cabrerizo M., Adjouadi M., Ayala M., Nunez K., Jayakar P., and Yaylali I. "Integrated Analysis of EEG Functional Brain Mapping Based on an Auditory-Comprehension 
Process Augmented Through Topograpgical Maps and a New Eigensystem Study",Brain Topography, Vol. 17 (3), pp. 151-163, 2005.

Calvagno G., Ermani M., Rinaldo R., and Sartoretto F., A multiresolution approach to spike detection in EEG. EEE International Conference on Acoustics Speech and Signal Processing, 2000, Vol. 6, pp. 3582-3585.

Davey B.K., Fright W.R., Carroll G.J., and Jones R.D., "Expert System Approach to Detection of Epileptiform Activity in the EEG," Medical and Biological Eng. \& Computing, 27:365 - 370, 1989.

Dingle A.A., Jones R.D., Caroll G.J., and Fright R.W., "A Multiscale System to Detect Epileptiform Activity in the EEG", IEEE Trans. on Biomedical Engineering, 40 (12), 1260-1268, 1993.

Ebersole J.S., Noninvasive Localization of Epileptogenic Foci by EEG Source Modeling, Epilepsia, 41(Suppl. 3), 2000: 24-33.

Eberhart R.C., Dobbins R. W., and Hutton L. V., "Chapter 7: Performance Metrics" in Neural Network PC Tools, Academic Press, pp. 161-177, 1990.

Ebersole J.S., EEG and MEG dipole source modeling. In Epilepsy: a comprehensive textbook, Engel J. Jr. and Pedley T.A, Eds. Philadelphia: Lippincott-Raven, 1997, pp. 919-935.

Frost, Jr., J. D., “Automatic Recognition and Characterization of Epileptiform Discharges in the Human EEG," Journal of Clinical Neurophysiology, 2(3): 231 - 249, 1985.

Geiger, L. R., and Harner, R. N. (1978): EEG patterns at the time of focal seizure onset. Arch. Neurol., 35:276-286.

Gevins A.S., and Remond A., (Eds.), Methods of analysis of brain electrical and magnetic signals. Handbook of Electroencephalography and Clinical Neurophysiology, Vol. 1, Elsevier, Amsterdam 1987.

Gevins, A. and Remonds, A. Handbook of Electroencephalography and Clinical Neurophysiology, 1987, Greenstein, B. and Greenstein, A. Color atlas of neuroscience, Neuroanatomy and Neurophysiology, Thieme Stuttgart New York, 2000.

Greenstein, B. and Greenstein, A. Color atlas of neuroscience, Neuroanatomy and Neurophysiology, Thieme Stuttgart New York, 2000.

Glover J. R., Raghavan N., Ktonas P.Y., and Frost J. D. Context-based automated detection of epileptogenic sharp transients in the EEG: Elimination of false positives. IEEE Trans. on Biomedical Engineering, 1989, vol. 36 (5), pp. $519-527$. 
Gonzalez, R. C., Woods, R. E., "Image Transforms," Digital Image Processing, AddisonWesley Publishing Company, 81 - 159, 1993.

Gotman J., and Wang L.Y., "State Dependent Spike Detection: Validation," Electroencephalography and Clinical Neurophysiology, 83: 12 - 18, 1992.

Gotman J., Practical use of computer-assisted EEG interpretation in epilepsy. Clinical Neurophysiology, 1985, vol. 2(3), pp. $251-265$.

Hellmann G., "Multifold features determine linear equation for automatic spike detection applying neural networks in interictal EcoG”, Clinical Neurophysiology, May 1999, vol. $11(5)$, pp. 887-894.

Hilborn R. C. Chaos and Nonlinear Dynamics. An Introduction for Scientists and Engineers, Second Edition, Oxford University Press, 2000.

Iasemidis, L.D., Barreto, A., Gilmore, R.L., Uthman, B.M., Roper, S.A. and Sackellares, J.C., "Spatiotemporal evolution of dynamical measures precedes onset of mesial temporal lobe seizures", Epilepsia, vol. 35 (Suppl 8), pp. 133, 1994.

Jayakar P., Patrick J.P., Shwedyk E., Seshia S.S., "Automated Rule Based Graded Analysis Of Ambulatory Cassette EEGs," Electroenceph. Clin. Neurophysiol., 72, pp. 165 - 175, 1989.

Jayakar P., Resnick T.J., Duchowny M.S., Alvarez L.A. Localization of epileptogenic foci using a simple reference-subtraction montage to detect small inter-channel time differences. J Clin Neurophysiol, 1991; vol. 8, pp. 212-215.

Jayakar P., Patrick J.P., Shwedyk E., and Seshia S.S. Automated rule based graded analysis of ambulatory cassette EEGs. Electroenceph Clin Neurophysiol, 1989, vol. 72, pp. $165-175$.

Kurth C., Gilliam F., and Steinhoff B. EEG spike detection with a Kohonen feature map. $J$ Annals of Biomedical Engineering, 2000, vol. 28(11), pp. 1362-1369.

Ko C.W., Chung H.W., "Automatic spike detection via an artificial neural network using raw EEG data", Clinical Neurophysiology, March 2000, vol. 111, no. 3, pp. 477-481(5).

Latka M., Was Z., Kozik A. and West B.J. Wavelet Analysis of Epileptic Spikes. Physical Review 2003, Vol. E 67, June issue, also in http://www.if.pwr.wroc.pl/ mirek/.

Le Van Quyen M., Martinerie J., Navarro V., Baulac M., and Varela F. Characterizing neurodynamic changes before seizure. Clin Neurophysiol, 2001, vol. 18(3), pp. 191-208. 
Martini, F. H. Fundamentals of anatomy \& physiology, fifth edition, Prentice Hall, New Jersey 07458, 2001.

Mirkovic N., Adjouadi M., Yaylali I., and Jayakar P. 3-D source localization of epileptic interictal spikes", Journal of Brain Topography, 2003, vol. 16 (2), pp. 111-119, Jan. 2003.

Moon T.K. and Stirling W.C., Mathematical Method and Algorithms for Signal Processing, Prentice Hall, 2000: 369-395.

Ochi A., Otsubo H., Chitoku S. , Hunjan A. , Sharma R., Rutka J. T., Chuang S. H., Kamijo K. , Yamazaki T., Snead O.C. Dipole localization for identification of neuronal generators in independent neighboring interictal EEG spike foci. Epilepsia, 2001, vol. 42(4), pp. 483-490.

Otsubo H., Shirasawa A., Chitoku S., Rutka J.T., Wilson S.B., Snead O.C., $3^{\text {rd }}$, "Computerized brain-surface voltage topographic mapping for localization of intracranial spikes from electrocorticography", Technical note, J Neurosurg 2001 Jun; 94(6), pp.1005-1009.

Popescu S. Trained wavelets used to detect epileptic spikes. Proc. of IEEE-SP International. Symposium, Time-Frequency and Time-Scale Analysis, New Jersey, 1998, pp. 285-288.

Proakis, J. G. and Dimitris, G. M. Digital signal processing - principles, algorithms and applications, Prentice Hall, Third Edition, 1996.

Shannon, C.E. A Mathematical Theory of Communiction. The Bell Systems Technical Journal, 27 (1948), 379-423 Daly, D.D., Pedley, A. Current Practice of Clinical Electroencephalography. Second Edition, pp. 263-267. 1997, Lippincott-Raven.

Soong, A.C.K., and Koles, Z.J., Principal-Component localization of the sources of the background EEG. Biomedical Engineering, IEEE Transactions, 1995, 42(1): 59-67.

Sprott, J. C. Chaos and Time-Series Analysis, Oxford University Press, 2003.

Tarassenko L., Khan Y.U, Holt M.R., "Identification of Interictal Spikes In the EEG Using Neural Network Analysis," IEE Proc. Science, Measurement and Tech., 145(6): 270-278, 1998.

Wilson S.B. and Emerson R. Spike detection: a review and comparison of algorithms. Clin Neurophysiol, 2002, Vol. 13 (12), pp.1873-1881. 
Yaylali, I., Kocak, H., Jayakar, P. Detection of seizures from small samples using non-linear dynamic system theory. IEEE Trans on Biomedical Engineering, 1996, Vol. 43 (7): 743751.

Zhukov, L., Weinstein, D., and Johnson, C. Independent component analysis for EEG source localization. IEEE Engineering in Medicine and Biology Magazine, 2000, 19(3):87-96. 


\section{MERCEDES CABRERIZO}

Born, Havana, Cuba

$1998-2000$

B.S., Computer Engineering, F.I.U., Miami, FL

$2001-2003$

M.S., Computer Engineering, F.I.U., Miami, FL

$2001-2006$

Research Assistant, Center for Advance Technology and Education (C.A.T.E.), F.I.U., Miami, FL

$2002-2005$

National Science Foundation Graduate Research Fellow

$2003-2006$

Ph.D. Candidate, Electrical Engineering, F.I.U., Miami, FL

\section{PUBLICATIONS AND PRESENTATIONS}

1. Cabrerizo M., Adjouadi M., Ayala M., Progress in Brain Mapping Research Chapter 5 titled: An Application of Eigensystem and Frequency Analysis in Brain Functional Mapping, NOVA Science, pp. 177-204., ISBN 1-59454-580-4, 2006.

2. Cabrerizo M., Adjouadi M., Nunez K., Yaylali I., and Jayakar P., "An Integrated Auditory-Comprehension Process Augmented through Topographical Maps and a New Eigensystem Study", Biomedical Sciences Instrumentation, Vol. 40, pp. 187192, 2004. Also presented at the $41^{\text {st }}$ Annual Rocky Mountain Bioengineering Symposium.

3. Cabrerizo M., Adjouadi M., Ayala M., and Nunez K., "An Inverse Solution To Functional Brain Mapping of the Auditory Process Using an Eigensystem Study", Journal of Inverse Problems in Science \& Engineering, Taylor and Francis, Vol. 14 (4), pp. 437-452, June 2006.

4. Cabrerizo M., Adjouadi M., Ayala M., Sesin A., "3D Epileptic Foci Detection Using an Integrated Neuro-Imaging Approach", WSEAS Transactions on Information Science and Applications, Vol. 2 (7), pp. 987-995, August 2005.

5. Cabrerizo M., Adjouadi M., Ayala M., Tito M., "Pattern Extraction in Interictal EEG Recordings towards Detection of Electrodes Leading To Seizures", Biomedical Sciences Instrumentation, Vol. 42, pp. 243-248. Also Presented at the Rocky Mountain Bioengineering Symposium, Terre Haute, Indiana, April 7-9, 2006.

6. Cabrerizo M., Adjouadi M., Ayala M., Nunez K., Prasana J. and Yaylali I. "Integrated Analysis of EEG Functional Brain Mapping Based on an AuditoryComprehension Process Augmented Through Topographical Maps and a New Eigensystem Study", Brain Topography, Vol. 17 (3), pp. 151-163, 2005.

7. Cabrerizo M., Adjouadi M., Yaylali I., Jayakar P., Nunez K., "A New Algorithm for the EEG Functional Brain Mapping Based on an Auditory-Comprehension Process ", Proceedings of the SCI 2003 - The 7th World Multiconference on Systemics, Cybernetics and Informatics, Orlando, Florida, USA, July 2003. http://www.iiisci.org/sci2003/ 
8. Adjouadi M., Sanchez D., Cabrerizo M., Ayala M., Jayakar P., Yaylali I., and Barreto A., "Interictal Spike Detection Using the Walsh Transform", IEEE Transactions on Biomedical Engineering, Vol. 51, No. 5, pp.868-873, May 2004.

9. Adjouadi M., Cabrerizo M., Ayala M., Sanchez D., Jayakar P., Yaylali I., and Barreto A. "Detection of Interictal Spikes and Artifactual Data through Orthogonal Transformations", Journal of Clinical Neurophysiology, Vol. 22(1), pp. 53-64, January/February 2005.

10. Adjouadi M., Cabrerizo M., Sanchez D., Ayala M., Jayakar P., and Barreto A., “A New Mathematical Approach based on Orthogonal Operators for the Detection of Interictal Spikes in Epileptogenic Data", Biomedical Sciences Instrumentation, Vol. 40 , pp. $175-180,2004$. Also presented at the $41^{\text {st }}$ Annual Rocky Mountain Bioengineering Symposium.

11. Adjouadi M., Cabrerizo M., Yaylali I., and Jayakar P., "Interpreting EEG Functional Brain Activity", IEEE Potentials, Vol. 23, Issue 1, pp. 8-13, Feb/March Issue 2004.

12. Adjouadi M., Sesin A., Ayala M., and Cabrerizo M., "Remote Eye Gaze Tracking System as a Computer Interface for Persons with Severe Motor Disability", pp. 761769, Springer-Verlag Lecture Notes in Computer Science, LNCS 3118, K. Miesenberger et al., Eds., 1191 pages, July 2004; ISBN: 3540223347, LC Control Number: 96221428. Also Presented at the ICCHP 2004, July 7-9, 2004, Paris, France.

13. Sesin A., Ayala M., Cabrerizo M., and Adjouadi M., "Jitter Reduction in Eye Gaze Tracking System and Conception of a Metric for Performance Evaluation", WSEAS Transactions on Computers, Issue 5, Vol. 3, pp. 1268-1273, 2004.

14. Adjouadi M., Cabrerizo M., Ayala M., "Seizing lesions in 3-D”, IEEE Potentials, Vol. 24, Issue 5, pp. 11-17, December/January, 2006.

15. Adjouadi M., Cabrerizo M., Ayala M., Núñez K., "An Approach to Functional Brain Mapping Using an Inverse Solution Based on the Principal Component Transform", Inverse Problems, Design and Optimization (IPDO) Symposium, Rio de Janeiro, Brazil, March 17-19, 2004.

16. Adjouadi M., Mirkovic N., Cabrerizo M., and Ayala M., "An Inverse Solution To 3D Source Localization Of Epileptic Foci Using An Integrated Multimodal NeuroImaging Approach", Inverse Problems, Design and Optimization (IPDO) Symposium, Rio de Janeiro, Brazil, March 17-19, 2004.

17. Adjouadi M., Cabrerizo M., Nuñez K., Ayala M., Yaylali I., Jayakar P., Sanchez D., "Interictal Spike Detection Using the Walsh Transform", Proceedings of the GSPX \& International Signal Processing Conference (CD), ISBN: 1009129, [online]: http://www.gspx.com, Dallas, TX, USA, March-April, 2003.

18. Adjouadi M., Sesin A., Ayala M., Cabrerizo M. "Configurable, Multimodal HumanComputer Interface System and Method", US Patent Application Serial No. $11 / 176,812$ July 2005. 